\title{
Gravidographics:
}

\section{A Health Lifestyle Segmentation of Pregnant Women in Transition to First Time Motherhood}

\author{
By \\ Jayne Krisjanous
}

A thesis submitted to Victoria University of Wellington in fulfilment of the requirements for the degree

of Doctor of Philosophy in Marketing

Victoria University of Wellington

January 2010 


\begin{abstract}
The purpose of this thesis is to contribute a new health psychographic/ lifestyle segmentation of pregnant women in New Zealand who are in transition to first time motherhood. It provides a better understanding of the make up of the market and the way it aggregates into health lifestyle segments. By using a wide selection of pre-existing and pregnancy related health and attitude dimensions, it complements existing understanding and classifications of pregnancy health lifestyle.
\end{abstract}

First, the study richly describes pregnancy health behaviour within a representative cohort of pregnant women in a transitional lifestyle using a holistic framework. It takes into account lifestyle as made up of a combination of factors and underlying motivations. Furthermore, it establishes the application of 'lifestyle' as it is used in marketing as legitimate for the study of a transitional health lifestyle. The research is cross disciplinary, and for that reason, integrates two perhaps seemingly disparate (marketing and health) understandings of lifestyle, using a somewhat novel approach. It is inclusive of high risk pregnant women and a much less oft studied group, normal, healthy or low risk women.

Second, a key component of this study is the seminal development of a segmentation typology that classifies women's health in pregnancy through a situation specific psychographic approach. This typology has been termed 'Gravidographics', and identifies segments of pregnant women, who have distinct or unique identifiers in regard to their pregnancy health lifestyle. This knowledge will be useful for health behaviour change through social marketing and also the design and targeting of products and services to specific groups of pregnant consumers.

A third focus of the study is the examination of health lifestyle within the concept of life stage transition. As pregnancy marks the transit into a new life stage, there are many adaptations that need to be made, and several underlying factors influence the way such a journey is approached and achieved.

The study is exploratory and the conceptual framework, derived predominantly from the marketing and health literature, guides the study and informs development of the data collection tools. Eight main research questions are explored. The study uses a survey design and is cross sectional, with quantitative 
enquiry the dominant logic. A survey using a self administered health psychographic questionnaire was distributed by Lead Maternity Carers to pregnant women in their care. A sample of 478 women was obtained for this stage of the study. Quantitative analysis was undertaken through descriptive statistics and bi-variate analyses. Secondly, two step clustering was undertaken to develop pregnancy health lifestyle clusters. Four health lifestyle segments were identified. These segments were then profiled against additional variables and data that led to rich descriptions of each segment.

Several managerial recommendations are made that will assist in the delivery and positioning of maternity health goods and services particularly in regard to healthcare marketing and segmentation. Secondly, recommendations for future marketing communication strategies targeting pregnant women are made. Through this work, the study's ultimate aim; making an academic contribution to knowledge that will lead to improved health outcomes for mother and baby, has been achieved. 


\section{ACKNOWLEDGEMENTS}

I would like to express my sincere gratitude to all the women who gave their time to contribute to this study. Without their generosity this study would not have been possible. I would also like to extend my warmest thanks to the Lead Maternity Carers who helped in the distribution of questionnaires and the support and time they gave me.

My supervisors Professor Peter Thirkell, Professor of Marketing, Victoria University of Wellington and Professor Maralyn Foureur, Professor of Midwifery, University of Technology, Sydney, have provided me with a great deal of guidance and support. Nokuthaba Sibanda also provided valuable input. I appreciate their wisdom immensely.

Many colleagues and friends have also supported me along the way. I would like to thank especially Beth Rose and Viv Shaw for never doubting that I would complete this journey. I also greatly appreciate the support and friendship of Jim Richard, Aaron Gazley, Joanna Scott-Kennel, Kate Daellenbach, Janet Carruthers and Robyn Maude. They have all provided me with unwavering support and encouragement. The forums at Victoria University I have been able to share in with fellow PhD students have also been enriching. The 'Critical Thinking in Marketing' seminar group in the School of Marketing and International Business and the PhD Schools at the Post Graduate School of Nursing and Midwifery have allowed me to interact with both marketing and nursing/midwifery colleagues. The benefit of both disciplines has allowed me to engage with fellow students who can only be described as a source of great joy and learning. It has been a privilege.

Lastly but not at all least, thanks to my family for being prepared to give up many of the things families usually do with their time together, to allow me to complete this thesis. 


\section{TABLE OF CONTENTS}

$\begin{array}{lc} & \text { Page } \\ \text { Abstract } & \mathrm{i} \\ \text { Acknowledgements } & \text { iii } \\ \text { Table of Contents } & \text { iv } \\ \text { List of Tables } & \text { xii } \\ \text { List of Figures } & \text { xv }\end{array}$

\section{CHAPTER 1. INTRODUCTION}

1.1. Introduction 1

1.2. Background 2

1.3. Research problem 3

1.4. Research purpose, objectives and research questions 4

1.4.1. Research Questions 5

1.5. Conceptualisation 5

1.6. Research Methodology 6

$\begin{array}{ll}\text { 1.7. Delimitations of the study } & 7\end{array}$

$\begin{array}{lr}\text { 1.8. Importance and value of the research } & 8\end{array}$

1.8.1. Importance of the research 8

$\begin{array}{ll}\text { 1.8.2. Importance of the research for academics } & 10\end{array}$

$\begin{array}{ll}\text { 1.8.3. Value of the research for practitioners } & 10\end{array}$

1.9. Chapter 1 Summary 11

\section{CHAPTER 2. LITERATURE REVIEW}

2.1. Introduction 12

2.2. Marketing framework: Healthcare marketing and social marketing 12

2.2.1. Healthcare marketing 13

2.2.2. Social marketing $\quad 14$

2.2.3. Section summary 16

$\begin{array}{ll}\text { 2.3. The context of pregnancy } & 17\end{array}$

2.3.1. Introduction 17

2.3.2. Historical influences 18

2.3.3. The New Zealand maternity care system 19

2.3.4. The maternity consumer 20

2.3.5. Pregnancy as a driver of consumer demand 22 


\subsubsection{Section summary}

2.4. Pregnancy and life stage transition 23

2.4.1. Introduction $\quad 23$

2.4.2. Household lifecycle models $\quad 24$

2.4.3. Transition theory $\quad 25$

$\begin{array}{ll}\text { 2.4.4. Section summary } & 30\end{array}$

2.5. Health and health lifestyle 31

2.5.1. Introduction 31

2.5.2. Lifestyle 31

2.5.3. Measuring lifestyle $\quad 32$

2.5.3.1. Psychographics 33

2.5.3.2. Methodological issues in lifestyle research 35

2.5.3.3. Choice of approach for the study 38

2.5.4. Measuring health and health lifestyle $\quad 39$

2.5.4.1. Health 39

2.5.4.2. Health psychographics 42

$\begin{array}{ll}\text { 2.5.5. Pregnancy health as a lifestyle } & 47\end{array}$

2.5.5.1. Consumer decision making and pregnancy health $\quad 47$

2.5.5.2. Pregnancy health psychographics 49

2.5.6. Section summary $\quad 52$

2.6. Components of a pregnancy health lifestyle 52

2.6.1. Introduction $\quad 52$

2.6.2. Pre-existing dimensions $\quad 53$

2.6.2.1. Demographics 53

2.6.2.2. Pre-existing values and traits 56

2.6.2.3. The circumstances around the pregnancy 61

2.6.3. Drivers of transition 63

2.6.3.1. Information 64

2.6.3.2. Pregnancy health attitudes 68

$\begin{array}{ll}\text { 2.6.3.3. Motivations and barriers } & 69\end{array}$

2.6.4. Physical and psycho-social dimensions of pregnancy 71

$\begin{array}{ll}\text { 2.6.4.1. Physical dimensions } & 71\end{array}$

2.6.4.2. Psycho-social dimensions $\quad 77$

2.6.5. Transition progress and outcomes 81

2.6.5.2. Transition markers $\quad 82$

2.6.6. A temporary or transformed lifestyle? $\quad 83$ 


\section{CHAPTER 3. CONCEPTUAL FRAMEWORK AND RESEARCH QUESTIONS}

3.1. Chapter introduction $\quad 87$

3.2. Background $\quad 87$

3.3. The Transitional Pregnancy Health Lifestyle conceptual framework 88

3.4. Framework dimensions, constructs and variables explained 91

3.4.1. Influencing Lifestyle Factors dimension $\quad 91$

3.4.1.1. Socio-demographic sub-domain 92

3.4.1.2. Pregnancy Circumstance sub-domain 93

3.4.1.3. Pre-existing Influences sub-domain 94

3.4.2. Life in Transition dimension 98

3.4.2.1. Transition Adaptation Adjusters sub-domain 98

3.4.2.2. The Transitional self sub-domain 101

3.4.2.3. The Transitional self (Physical) 102

3.4.2.4. The Transitional self (Psycho-social) 105

3.4.3. Adaptation Dimension 106

3.4.3.1. Transition Success sub-domain 106

$\begin{array}{ll}\text { 3.5. Conceptual framework summary } & 107\end{array}$

3.6. Comment on additional factors considered in the design $\begin{array}{ll}\text { of the framework } & 110\end{array}$

3.7. Use of the conceptual framework to guide analysis 111

3.8. Chapter 3 summary 112

\section{CHAPTER 4 STUDY DESIGN}

4.1. Introduction 113

4.2. Study approach 113

4.3. Overview of the design 114

4.4. Ethics and access 114

$\begin{array}{ll}4.5 \text { Consultation with Māori } & 115\end{array}$

4.6. Feedback provision for participants 116

$\begin{array}{ll}\text { 4.7. Quantitative phase of the research } & 116\end{array}$

$\begin{array}{ll}\text { 4.7.1. Questionnaire design } & 116\end{array}$ 
4.7.1.1. Tool development 116

4.7.1.2. Considerations specific to the context 119

4.7.1.3. Item and scale generation 119

4.7.1.4. Type of response 122

4.7.1.5. Presentation and formatting 123

4.7.1.6. Questionnaire refinement and verification 124

4.7.1.7. Pilot testing 125

4.7.2. Final questionnaire measures $\quad 125$

$\begin{array}{ll}\text { 4.7.2.1. Socio-demographics } & 126\end{array}$

4.7.2.2. Pregnancy Circumstance 127

4.7.2.3. Pre-existing Influences 128

4.7.2.4. Transition Adaptation Adjusters 131

4.7.2.5 The Transitional Self (Physical) 135

4.7.2.6. The Transitional Self (Psycho-social) 138

4.7.2.7. Transition Success 140

4.7.3. Survey implementation and data collection 141

4.7.3.1. Access arrangements 141

4.7.3.2. Sampling design 143

4.7.3.3. Distribution 146

4.7.3.4. Changes within the external environment 146

4.7.3.5. Follow up letters and contact 147

4.7.3.6. Calculation of response 148

4.7.4. Data analysis 150

4.7.4.1. Factor analysis 150

4.7.4.2. Descriptive analysis and tests of association 151

4.7.4.3. Cluster analysis and profiling 152

4.7.4.3.i. Sub-clustering phase 153

4.7.3.3. ii. Gravidographic clustering phase $\quad 154$

4.8. Chapter 4 Summary 155

\section{CHAPTER 5. DATA ANALYSIS AND RESULTS}

5.1. Introduction 156

5.2. Quantitative results (Preliminary information) 156

5.2.1. Data entry and cleaning 155

5.2.2. Questionnaire validation and excluded questionnaires 156 
5.2.3 Treatment of extreme and missing values

5.2.4. Tests for normality

5.2.5. Sample size and power

5.2.6. Exploratory factor analysis and scale validation 158

5.2.7. Cronbach's alphas 156

5.2.8. Presentation of results 158

5.2.9. Sample representativeness 158 159

5.3. Exploratory factor analysis 159

5.3.1. Confirmatory Factor Analysis (CFA) of existing scales) 159

5.3.2. Exploratory Factor Analysis (EFA) of new scales 163

5.3.3. Performance of scales 165

5.4. Descriptive results and bi-variate analyses

5.4.1. Socio-demographic descriptive results 166

5.4.1.1. Age, Ethnicity and Education 167

5.4.1.2. Living and Accommodation 170

5.4.1.3. Primary language at home

5.4.1.4. Work and Income 171

5.4.1.4. Media use

5.4.1.5. Summary of socio-demographic profile and representation

5.4.2. Pregnancy Circumstance descriptive results

5.4.2.1. Current and previous pregnancies

5.4.2.2. Arranging pregnancy care

5.4.2.3. Risk

5.4.2.4. Summary of descriptive results for Pregnancy Circumstance 177

5.4.3. Pre-existing Influences descriptive results

5.4.3.1. Pre-existing Health, Mastery and Achievement and Other values, traits and beliefs

5.4.3.2. Summary of pre-existing influences descriptive results

5.4.4. Transition Adaptation Adjusters descriptive results

5.4.4.1. Information Acquisition and Information Processing and Utilisation 
5.4.4.3. Summary of Transition Adaptation Adjusters descriptive results 191

5.4.5. The Transitional Self descriptive results 191

5.4.5.1. Nutrition 192

5.4.5.2. Exercise and rest 194

5.4.5.3. Substance use 196

5.4.5.4. Risk reduction 199

5.4.5.5. Support 200

5.4.5.6. Coping 201

5.4.5.7. Experiencing the Pregnant Body 202

5.4.5.8. Satisfaction with living arrangements 203

5.4.5.9. Summary of the Transitional Self descriptive results 204

5.4.6. Transition Success descriptive results 204

5.4.6.1. Wellbeing, Attachment and Orientation to the future 205

5.4.6.2. Summary of Transition Success results 206

5.4.7. Descriptive results summary 206

$\begin{array}{ll}\text { 5.5. Cluster analysis } & 207\end{array}$

5.5.1 Stages of cluster procedure 207

5.5.1.1. Information sub-cluster (Transition Adjusters sub-domain) 208

5.5.1.2. Other Attitudes and Drivers sub-cluster (Transition Adjusters sub-domain 212

5.5.1.3. Nutrition construct sub-cluster (from The Transitional Self sub-domain) 217

5.5.1.4. Exercise and Rest construct sub-cluster 223

5.5.1.5. Pregnancy Health Behaviour sub-cluster (Physical Health Behaviour sub-domain) 226

5.5.1.6.Psychosocial sub-cluster 235

5.5.1.7. Transition Success sub-cluster (from the Adaptation sub-domain) 236

5.5.2. Summary of sub-clustering phase 242

5.5.3. Gravidographic clustering 242

5.5.3.1. Gravidographic cluster analysis results 243

5.5.3.2. Gravidographic cluster descriptions 256

5.5.3.3. Evaluating the adequacy of the cluster solution 257

5.5.3.4. Checking stability of Gravidographic clusters 257 
5.5.3.6. Description of Gravidographic clusters by profiling variables

5.6. Summary of clustering phase

\section{CHAPTER SIX. DISCUSSION AND CONCLUSION}

6.1. Introduction 272

6.2. Discussion of findings 272

6.2.1. A description of the Health Lifestyle of the pregnant nulliparous market 272

6.2.1.1. Influencing Lifestyle Factors. 273

6.2.1.2. Transition Adaptation Adjusters 273

6.2.1.3. The Transitional Self 281

6.2.1.4. Transition Success 288

6.2.2. Segmentation 289

6.2.2.1. Formation of segments and segmentation patterns 289

6.2.3. The 'Casual Recipe' of Pregnancy Health Lifestyles 292

6.2.4. Successful transition 293

6.2.5. Pregnancy health changes and other health transitions 294

6.2.6. Targeting pregnant audiences for health communications 295

6.2.7. Comments about the conceptual framework and theories used. 298

6.2.8. Summary/main points of discussion 298

6.3. Study limitations $\quad 300$

6.3.1. Generalisation and representation 300

$\begin{array}{ll}\text { 6.3.2. Distribution and access } & 301\end{array}$

6.3.3. Boundaries of study 303

6.4. Recommended future research 303

6.4.1. Further development of the quantitative tool 303

6.4.2. Wider country, cultural and consumption assessment 303

6.5. Theoretical contribution and managerial/practise

$\begin{array}{ll}\text { recommendations } & 304\end{array}$

$\begin{array}{ll}\text { 6.5.1. Theoretical } & 304\end{array}$ 
6.5.1.1. The Transitional Health Lifestyle 304

6.5.1.2. Gravidographics 304

6.5.2. Managerial and practice 305

6.5.2.1. Service design 305

6.5.2.2. Information and source credibility of LMCs 305

6.5.2.3. Consumer decision making 305

6.5.2.4. Knowledge of pregnancy health in the wider

Community 306

6.5.2.5. Social marketing campaigns and health

Promotion communication 307

6.5.2.6. Making health choices easier for women 307

6.5.2.7. Contemporary maternity markets and technology 308

$\begin{array}{ll}\text { 6.6. Conclusion and final remarks } & 308\end{array}$

$\begin{array}{lr}\text { REFERENCES } & 310\end{array}$ 


\section{LIST OF TABLES}

Table 2.1: Health Psychographics; Empirical and Conceptual Studies

Page

Table 3.1: Dimensions, Sub-domains and Constructs and Attributes

45

Table 3.2: Dimensions, Sub-domains, Constructs /Attributes and Variables

91

Table 4.1:Pre-existing Influence Measures

106

Table 4.2: Pregnancy Circumstance Measures 128

Table 4.3: Socio-Demographic Measures

Table 4.4: Pregnancy Transition Adjusters Measures 130

Table 4.5: Transitional Self (Physical) Measures

Table 4.6: Transitional Self (Psychosocial) Measures 136

Table 4.7: Transition Success Measures 139

Table 4.8: Distribution by LMC Type and Region

Table 5.1. Pre-pregnancy health level scale

Table 5.2. Health consumerism scale 160

Table 5.3. Environmentalism scale 160

Table 5.4. Concern for physical appearance scale 160

Table 5.5. Religiosity scale 160

Table 5.6. Trait anxiety scale

Table 5.7. Sense of mastery scale

Table 5.8. Health Locus of Control (HLOC): Internal scale 161

Table 5.9. Health Locus of Control (HLOC): Fate scale

Table 5.10. Health Locus of Control (HLOC): Powerful Others scale

Table 5.11. Familism scale

Table 5.12. Maternal-fetal attachment scale

Table 5.13. Information receipt interest scale

Table 5.14. Information outcome beliefs scale

Table 5.15. Information change result scale

Table 5.16. Pregnancy health effort and motivation scale

Table 5.17. Self-efficacy for making health changes scale

Table 5.18. Good nutrition effort scale

Table 5.19 Activity interference scale

Table 5.20 Pregnancy state anxiety scale

Table 5.21 Life situation state anxiety scale

Table 5.22. Weight concerns scale 
Table 5.25. Perception of wellness scale

Table 5.26. Age, Ethnicity and Education

Table 5.27 Age by Ethnicity (reduced categories)

Table 5.28. Age by Education

Table 5.29. Living and Accommodation

Table 5.30. Work and income

Table 5.31. Household Yearly Income by Ethnicity

Table 5.32. Media use

Table 5.33. Internet Usage* by Yearly Household Income Groups

Table 5. 34. Feelings about the Pregnancy, Pregnancy Dates and Other Experiential Factors

Table 5.35. Rating as a life event: Pearson's correlations

Table 5.36. First awareness by Pregnancy intendedness

Table 5.37 'Pregnancy intendedness' by 'Evaluation of pregnancy worth'

Table 5.38. Arranging pregnancy care

Table 5. 39. Level of risk

Table 5 40. Level of risk by LMC

Table 5.41. Level of risk by Age group

Table 5 42. Pre-existing Health, Mastery and Achievement and Other values, traits and beliefs

Table 5.43. Kahle's List of Values (Universal values)

Table 5.44. Pre-existing influences: Pearson's correlations

Table 5 45. Information Acquisition and Information Processing and Utilisation

Table 5.45a. Antenatal classes* and Topic importance

Table 5.46 Affinity for technology, Pregnancy Health Locus of Control, Pregnancy health motivation and effort, Achievement motivation

Table 5 48. Influences for change to improve health in pregnancy

Table 5.49. Nutrition attitudes, Nutrition practice and Perceived barriers to good nutrition

Table 5.50. Avoids risky foods and Intake against 
Table 5.53. Exercise attitudes, Barriers and interference and Sleep and Rest

Table 5.54. Exercise practices: Planning and Patterns

Table 5 55. Frequency of exercise and 'Has an exercise plan

Table 5.56. Attitude to medication use in pregnancy and medication use

Table 5.57. Tobacco use

Table 5.58. Smoking and pregnancy: Māori vs Non Māori

Table 5.59. Alcohol intake

Table 5.60. Support importance and Perceived support

Table 5.61. Type of support desired

Table 5.62. Coping, Anxiety, Emotions and reactions and Relaxation

Table 5.63. Pregnant body image, Appearance interest and

Celebrity attraction

201

Table 5.64. Transitional self: Pearson's correlations

202

Table 5.65. Satisfaction with accommodation

Table 5.66. Wellbeing, Attachment and Orientation to the future

Table 5.67. Transition success: Pearson's correlations

Table 5.68. Information sub-cluster scale and item mapping to variables and constructs

Table 5.69 Information sub-cluster distribution

Table 5.70. Information sub-cluster: Continuous Measures

Table 5.71. Information sub cluster items/scales put forward to Gravidographic cluster stage

Table 5.72. Other Attitudes and Drivers sub-cluster Scale and item mapping to variables and constructs

Table 5.73. Other Attitudes and Drivers sub-cluster distribution

Table 5.74. Other Attitudes and Drivers sub-cluster continuous measures

Table 5.75. Other Attitudes and Drivers sub-cluster interpretation

Table 5.76. Other Attitudes and Drivers sub-cluster items/scales

put forward to Gravidographic cluster stage

Table 5.77. Nutrition construct sub-cluster scale and item mapping to variables

Table 5.78. Nutrition construct sub-cluster distribution

Table 5.79. Nutrition Sub-Cluster distribution Folic acid intake

Table 5.80. Nutrition construct sub-cluster continuous variables 
Table 5.81. Nutrition construct sub-cluster interpretation

Table 5.82. Nutrition construct sub-cluster items and scales put forward to Gravidographic cluster phase

Table 5.83. Exercise and Rest construct sub-cluster scale and item mapping

Table 5.84. Exercise and Rest construct sub-cluster distribution

Table 5.85. Exercise and Rest construct sub-cluster: Frequency of exercise

Table 5.86. Exercise and Rest construct sub-cluster continuous measures

Table 5.87. Exercise and Rest construct sub-cluster interpretation

Table 5.88. Nutrition construct sub-cluster items and scales put

forward to the Gravidographic cluster phase

Table 5.89. Pregnancy Health Behaviour sub-cluster: Scale and item

mapping to variables

Table 5.90. Pregnancy Health Behaviour sub-cluster:

Sub-cluster distribution

Table 5.91. Pregnancy Health Behaviour sub-cluster:

Smoking patterns since pregnant

Table 5.92. Pregnancy Health Behaviour sub-cluster distribution: Alcohol

Table 5.93. Pregnancy Health Behaviour sub-cluster Frequency of exercise

Table 5.94. Pregnancy Health Behaviour sub-cluster:

Over-the-counter (OTC) medication taken

Table 5.95. Pregnancy Health Behaviour sub-cluster: Continuous measures:

Table 5.96. Pregnancy Health Behaviour sub-cluster interpretation

Table 5.97. Pregnancy Health Behaviour sub-cluster items and

scales put forward to Gravidographic cluster stage.

Table 5.98. Psychosocial SC scale and item mapping to variables

and constructs

Table 5.99. Psychosocial SC distribution

Table 5.100. Psychosocial SC continuous measures

Table 5.101. Psychosocial SC interpretation

Table 5.102. Psychosocial SC items and scales put forward

to Gravidographic stage

Table 5.103. Transition Success SC scale and item mapping to variables and constructs

Table 5.104. Transition Success SC distribution 
Table 5.105. Transition Success SC continuous measures

Table 5.106. Transition Success SC interpretation 239

Table 5.107. Transition Success SC items and scales put forward to Gravidographic stage 239

Table 5.108. Summary table of final variables for Gravidographic clustering 240

Table 5.109. Gravidographic (GC) Cluster Membership 243

Table 5.110. GC Pregnancy Intendedness 244

Table 5.111. GC Evaluation of pregnancy worth

Table 5.112. GC Information acquisition and Information processing and Utilisation 245

Table 5.113. GC Other Pregnancy Attitudes and Drivers

Table 5.114. GC Good nutrition effort 248

Table 5.115. GC Reducing nutrition risk: Folic acid intake 248

Table 5.116. GC Exercise and Rest 'Perceived interference level' and 'Follows recommendations

Table 5.117. GC Exercise rate 250

Table 5.118. GC Smoking patterns since pregnant 250

Table 5.119. GC Alcohol patterns since pregnant 251

Table 5.120 Wearing Safety Belt and Care with Dental Hygiene

Table 5.121: GC Pregnancy State Anxiety, Weight Concern and Pregnant body image

Table 5.122. GC Transition Success

Table 5.123. GCs by Age

Table 5.124. GCs by Ethnicity 258

Table 5.125. GCs by Domicile by size of region 259

Table 5.126. GCs by Educational group 259

Table 5. $127 \mathrm{GC}$ by Occupation 260

Table 5.128. GCs by Household income 260

Table 5.129. GCs by Employment status 261

Table 5.130. GCs by Media Use 261

Table 5.131. GCs by Internet Usage

Table 5.132. GCs by Living with

Table 5.134. GCs by Contact with Baby's Father 262

Table 5.135. Importance of mother for support 263

Table 5.136 . GCs by Happy with living accommodation 
Table 5.137. GCs by Weeks aware of pregnancy

Table 5.138. GCs by Original Intentions Pregnancy 264

Table 5.139. GCs by Type of LMC care

264

Table 5.140. GCs by Higher risk level

265

Table 5.141. GCs by Ovulation/Fertility medication

265

Table 5.142. GCs by Artificial insemination

265

Table 5.143. GCs by IVF

266

Table 5.144. GCs by Pre-existing influences

267

Table 5.145. Gravidographic Clusters and Non-significant

Cross-tabulations and ANOVAs

269 


\section{LIST OF FIGURES}

Page

Figure 1.1. Figure 1.1 Study Research Domain 2

Figure 3.1: Conceptual Framework: The Pregnancy Health Lifestyle 88

Figure 3.2: Conceptual Framework: The Transitional Pregnancy

Health Lifestyle by Sub-domain, Constructs and Attributes and Variables 109

Figure 4.1: Clustering Procedure Leading to the Identification

of Gravidographic Segments

Figure 5.1. Nutrition construct within sub-cluster \% of Folic acid intake

Figure 5.2. Exercise and Rest construct within sub-cluster: Frequency of exercise

Figure 5. 3. Pregnancy Health Behaviour within sub-cluster \%:

Smoking patterns since pregnant

Figure 5 4. Pregnancy Health Behaviour within sub-cluster distribution:

Alcohol Patterns since pregnant

Figure 5.5. Pregnancy Health Behaviour within sub-cluster \%:

Frequency of exercise

Figure 5.6. Pregnancy Health Behaviour within sub-cluster \%:

OTC medication taken

Figure 5.7. Pregnancy Circumstance Gravidographic Cluster variables

Figure 5.8. GC within cluster \%: Pregnancy intendedness

Figure 5.9. GC within cluster \%: Evaluation of pregnancy worth

Figure 5.10. GC within cluster \%: Folic acid intake 
Figure 5.11. GC Within cluster \%: Frequency of exercise

Figure 5.12. GC within cluster \%: Smoking patterns since pregnant

Figure 5.13. GC within cluster \%: Alcohol patterns since pregnant 


\section{CHAPTER 1 INTRODUCTION}

\subsection{INTRODUCTION}

Healthcare marketing first came to the attention of marketers in the 1970's (Willcocks \& Conway, 1998; Andreasen, 2003). Today, health research and behaviour change through social marketing is of great interest to marketing, as it joins with other disciplines to focus on issues that cause problems in regard to health and quality of life, for both individuals and societies. Efforts to reduce costs (both human and financial), borne by health systems and caused through health problems such as obesity, smoking and alcohol abuse, are major drivers of public health and social marketing interventions. Arguably, health lifestyle factors are associated with many health problems specific to pregnancy: excess alcohol consumption (leading to fetal alcohol syndrome), increased risk as a result of demographic/social situations (adolescent pregnancy) and potential danger due to pre-existing physical conditions (gestational diabetes and a 'large for dates' baby).

Health behaviours that make up a person's health lifestyle can vary in a number of ways. They can be health promoting (good nutrition, regular exercise), risk enhancing (smoking, sedentary lifestyle), or carried out for health protection (regular medical check ups, breast cancer screening). They may comprise multiple health and non-health social factors (alcohol consumption) or strong cultural components (dietary habits) (Steptoe \& Wardle, 2001). Emphasis on the role of a 'healthy lifestyle', including altering lifestyle behaviours (such as smoking and weight control), in order to promote and maintain health has increased substantially in recent years (Kraft \& Goodell, 1993). Health lifestyle is a legitimate way to view the structural changes, physiological responses and the psychosocial dimensions of pregnancy. There is no doubt that the lifestyle engaged in by pregnant women, despite pregnancy being a transitional period in a woman's life, has distinct and identifiable patterns of behaviour attached to it, which are repeated and endured over a period of time (Tulman \& Fawcett, 2003; Chomitz, Cheung, \& Lieberman, 1995). Therefore, the application of 'lifestyle' as it is used in marketing is legitimate for the study of a transitional health lifestyle.

Maternal health during pregnancy and the improvement in maternal-fetal outcomes, are also a focus for the health disciplines. Whilst maternal-fetal mortality rapidly reduced in the Western world during the $20^{\text {th }}$ century, morbidity for both mother and baby, caused by preventable maternal behaviours and 
factors (for example, smoking, alcohol, poor nutrition and lack of social support), still impacts significantly on health outcomes.

Increasingly, maternal health practices are recognised as a key contribution to the condition of a baby at birth, with certain practices associated with a high degree of risk for a baby. Building on what became known as the 'The Barker Hypothesis', from work by Barker (1986), recent research points to health practices and the supply of fetal nutrients in-utero as determinant in key health factors, such as the development of heart disease and obesity in later adult life. Vickers, Breier, McCarthy \& Gluckman (2003) report an important link between the in-utero experience and later metabolic and cardiovascular disease, with increasing evidence that, through a process of 'fetal programming', conditions in the fetal environment have long term consequences on metabolic and endocrine patho-physiology in adult life (Vickers et al., 2000; De Boo \& Harding, 2006; Kunz, 2007).

\subsection{BACKGROUND}

This thesis has been inspired by author experience in both marketing and health fields. Maternity care providers have in-depth knowledge of their clients' health situations and needs and must build an effective one-to-one relationship that instils client trust and confidence, often within a short period of time. Marketing has much to offer maternity health professionals and marketers alike, as it provides in-depth knowledge of consumer behaviour and approaches to aggregating consumers into meaningful groupings for the purposes of targeting, information based initiatives and interventions. Other disciplines, such as medicine, predominantly contribute to knowledge about the causation and manifestation of diseases, direct clinical observation and the devising of treatment protocols. Marketing is concerned with understanding the antecedents of consumer decisions around and within these complex situations. Therefore,

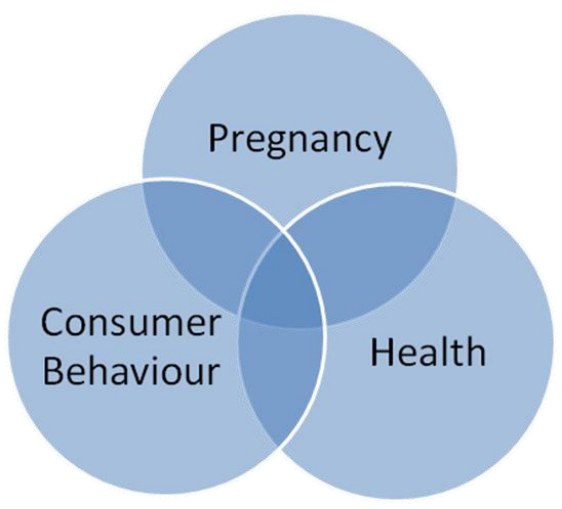
marketing tools, such as segmentation and communication, complement the goals of improving health by understanding the makeup of different consumer groups, in regard to attitudes, behaviours and responses to various communications strategies. The research domain for this study is within the intersection of three bodies of knowledge, pregnancy, health 
and consumer behaviour, as seen in Figure 1.1. There is extensive scope for cross fertilisation between the disciplines that to date has not been fully taken advantage of in order to more fully understand the behaviour of pregnant women. Identifying how both health promoting and non-health promoting behaviours may group together to comprise a particular lifestyle, provides valuable insights for all the disciplines. Furthermore, both health professionals and social marketers attempt to influence and change health and social behaviours to achieve better outcomes through persuasion based communication, whether on an individual level or through mass media delivered campaigns.

As pregnant consumers engage in a highly specific domain of exchange and consumption, it would seem that this group of consumers is relatively homogeneous. However a marked degree of diversity in demography, health status and circumstances in which pregnancy occurs exists in this market (Ministry of Health, 2004). Furthermore, with consumer heterogeneity and several options for maternity care particularly in New Zealand present (Ministry of Health), it stands to reason that significant variation in health circumstances and lifestyles amongst consumers might be found.

Pregnancy healthcare providers (particularly midwives and doctors) work closely with their clients to achieve the goal of a healthy outcome for mother and baby. In order to do this effectively, both parties need to have common expectations, mutual desires and effective strategies planned. In order to achieve a high degree of market orientation, knowledge of consumer attitudes and motivations that drive decisions and behaviours become extremely beneficial.

\subsection{RESEARCH PROBLEM}

The main problem addressed by this study is that although much is known about pregnancy health in regard to single behaviours and lifestyle factors that influence it, there is little research that integrates a range of health influences, attitudes and behaviours to provide a segmentation system by which maternity consumers can be understood and organized holistically.

In addition, this work proposes pregnancy as a transitional lifestyle (particularly in anticipation of a first baby) and as an event that bridges two life stages. It is important to know when and how the journey through this transition occurs, and how changes in attitudes or motivations lead to stable, positive or negative 
changes in behaviour. Whilst much has been undertaken on how a person grows into the motherhood role from a psycho-social perspective (for example, Mead, 1964; Roy, 1997), there is little that provides a holistic overview of the pregnancy course (whether planned or unplanned), particularly during the early stages of pregnancy. If certain health behaviours are to be reshaped to achieve better outcomes for mother and baby, the entire pregnancy course is important, especially given that some critical physiological growth for a baby is dependent on the maternal environment in pre-conception and early stages of pregnancy.

\subsection{RESEARCH PURPOSE, OBJECTIVES AND RESEARCH QUESTIONS}

With the above in mind, the purpose of this exploratory study was to examine the health behaviour of pregnant nulliparous (no previous birth) New Zealand women in transit toward their first birth and motherhood (entry into Full Nest 1 of The Household Lifecycle Model) from a health psychographic perspective. This thesis proposes that a psychographic market segmentation approach is a useful addition to knowledge that can be used within theoretical and practitioner fields and accomplishes significant first steps towards achievement of a robust psychographic typology. The outcome, a 'Gravidographics' typology of nulliparous pregnant women, complements existing methods used to understand the pregnancy market in order to meet the goods and services desired.

The study had three main objectives. The first objective was to comprehensively describe the market in regard to collective values, attitudes, health practices and other factors that comprise the pregnant health lifestyle. This provides better understanding of the meaning and interaction of underlying variables that make up this lifestyle. The second objective was to undertake a health psychographic/lifestyle segmentation and aggregate the market into smaller homogenous health lifestyle groups across selected health dimensions. Identifying segments with both health promoting pregnancy behaviours and those with potentially problematic health attitudes and behaviours can assist in health maintenance/promotion and campaigns targeting health promotion and/or behaviour change. The third objective was to evaluate this gestational period within the framework of a transitional lifestyle so as to identify the main drivers of transit in regard to health behaviour through this period. As part of that objective, evaluation of factors leading to what could be regarded as a successful transition was also undertaken. To the extent possible within the design, the 
study also sought to develop a better understanding of the nature of pregnancy as a health trajectory or turning point within usual life patterns.

\subsubsection{RESEARCH QUESTIONS}

The following are the research questions the study addresses. The research questions are exploratory in nature and aim to weave multiple strands of pregnancy health lifestyle together. As the questions are not specific to one relationship between two or more constructs (as would be the case in a hypothesis testing model), they are not assigned to one particular dimension of the framework only.

1. How can the pregnant nulliparous market be described in regard to collective values, attitudes, health practices and additional factors that make up the pregnant health lifestyle?

2. Do health behaviours of pregnancy cluster in meaningful ways and what are the underlying factors that best characterise the segments identified?

3. Does information as a transition adjuster play a primary role in the way a pregnancy health lifestyle is enacted?

4. Are there 'causal recipes' that lead to certain health behaviour styles?

5. Is 'success' in pregnancy transition associated with any particular lifestyle group over another?

6. What observations can be made in regards to pregnancy as a transitional health life stage, and the extent to which changes made are likely to be permanent or for the transitional period only?

7. Are the health behaviour changes made during pregnancy transition reflective of all life stage transitions, or are there factors that are unique?

8. Assuming clusters can be meaningfully identified, how can each be reached for effective health management, social marketing and health promotion messages that would lead to the adoption of health behaviours beneficial for both mother and baby?

\subsection{CONCEPTUALISATION}

The principles of segmentation theory underpin the conceptualization of this study. Market segmentation is used widely to manage diverse customer needs and to target marketing resources (Weinstein, 2004; La Placa, 1997; McDonald \& Dunbar, 2004) by aggregating consumers into distinct groupings, where similarity exists along nominated dimensions (Kalwani \& Morrison, 1977; Mahajan \& Jain, 
1978). This study uses a psychographic segmentation approach, which divides customers across dimensions such as values, and Activities, Interests and Opinions (AIO).

The study drew on existing knowledge from several other disciplines. The health lifestyle determined in pregnancy is influenced by specific antecedent factors, including socio-demographics, existing values, traits and beliefs, and the circumstance in which pregnancy occurs. In order to transit through pregnancy, changes and adjustments are made to many health practices and behaviours; for example, altering one's diet to cope with nausea and vomiting in early weeks, avoiding foods known to be risky during pregnancy, and seeking emotional support from a partner, are all indicators of adjustment to a new situation.

The conceptual framework developed for the study incorporates elements considered key influences in the pregnancy health lifestyle, including information acquisition and processing as a driver of decision making that leads to adaptation and change. In order to adjust to an altered state, information must be acquired, processed and responded to. As a result, the many facets of a health lifestyle are brought together and enacted within constraints and resources available, with the goal of realising the birth of the baby and the end of the pregnancy. For some, care of health may be of upmost importance, whilst for others it may be seen as a lesser or seemingly unattainable goal.

Focusing on a transitional health state, the study also addressed understanding of progress, adaptation and change and how this translates into successful transition. The concept of success in transition is not easy to define given that the outcome of the pregnancy (that is, birth) is not known and conventional measures that do exist (for instance, labour and birth progress and events, condition at birth and an uneventful post-natal period) are not measured in the study.

\subsection{RESEARCH METHODOLOGY}

In order to achieve the specified objectives and explore the conceptual framework proposed, the previously specified research questions were pursued. A survey design was used to operationalise the conceptual framework. 
The survey phase was executed through a context specific psychographic questionnaire. This consisted of a large set of items and scales designed to examine the influencing variables, transition adjusters (motivations, decision making process, information acquisition and use), and components of the lifestyle itself, that had been drawn from the literature. Measures, assessing the extent to which the transition being undertaken could be seen as successful or not, were also created. The end result was identification of specific 'Gravidographic' segments, including full profiling of the segments to allow clear identification of relevant attributes for health marketers and policy makers.

\subsection{DELIMITATIONS OF THE STUDY}

Health is complex and hence a multi-disciplinary approach was used. The researcher had knowledge of both the marketing and health disciplines which enabled a high degree of integration and synthesis of perspectives from a range of sources, including marketing, health, psychology and gender studies. The study also drew on relevant theoretical perspectives for a new view of pregnancy health lifestyle. These perspectives included, The Household Lifecycle Model, psychographics, transition theory, consumer decision making, and health behaviour/change models from social marketing.

For the purposes of this thesis, the pregnancy consumer market included women only, although it is acknowledged that childbearing events impact on all members of a family unit (or whanau) and almost all women experience childbearing within a wider support network. Many consumption decisions made in pregnancy are likely to be made by a woman in consultation with others members of her family or household. However, at an initial stage of conceptualisation it was believed that focusing on a woman's pregnancy health lifestyle was a very good first step. The study was also confined to nulliparous (no previous viable birth) women only. Although every pregnancy is a new transition journey in some respects, first time transition is believed to demand more new learning and significant adjustment than any subsequent pregnancy.

The study included both low risk and high risk pregnant women. High risk can be determined by medical diagnosis (whether pre-existing or pregnancy induced), but also includes high social risk (for example, poor living circumstance). It is acknowledged that in many situations, high social risk during pregnancy is correlated with certain life patterns and choices and engagement in non-health 
promoting behaviours that impact negatively on health outcomes (Chomitz et al., 1995). The worth of studying the lifestyle of low risk ('normal') women is also supported by several authors who note that, despite low risk pregnancy comprising the majority of all pregnancies, it is high risk situations that receive the most research focus (Smith, 1999; Burrows \& Williamson, 2004; Bondas \& Eriksson, 2001). Therefore, knowledge that leads to understanding of how health is maintained and enables women to make health adjustments during a normal pregnancy can be conceived of as a worthwhile aim to meet customer needs. This knowledge is also valuable as research that leads and informs health behaviour change due to risky behaviours. Medical focus on risk and disease can be over represented in pregnancy studies, leaving a corresponding gap in understanding healthy low risk women and the choices they make (Burrows \& Williamson, 2004; Bondas \& Eriksson, 2001).

Data for this pregnancy health lifestyle study has been sourced from New Zealand women. Although there are some factors in the New Zealand delivery of maternity services that are unique, (for instance $70 \%$ of primary births are attended by midwives without medical supervision; Ministry of Health, 2004), women in other countries (especially Western) are likely to be affected by the same influencing variables on health as New Zealand women (including health consumerism, media proliferation, demographic shifts and increased access to information).

Whilst the study's context is lifestyle during transition into Full Nest 1, its focus is confined to factors pertaining to health-related consumption only. Pregnancy lifestyle is made up of additional dimensions and purchases required to accommodate a new anticipated lifestyle (such as furniture and clothing purchases for the baby). These consumption related activities are, for the most part, not explored in this study.

\subsection{IMPORTANCE AND VALUE OF THE RESEARCH}

\subsubsection{IMPORTANCE OF THE RESEARCH}

Marketing involvement in health is now an important component of many healthcare systems (Willcocks \& Conway, 1998; Andreasen, 2003). By applying a marketing approach (psychographic segmentation) to the context under investigation, new knowledge can be gained about the makeup of the target groups in this market. Ideas discussed in this thesis will be of interest to 
audiences who wish to understand the needs of health consumers better. Improvements in quality of service and cost efficiencies are the goals of all healthcare providers, clinicians, and marketers alike. Furthermore, many countries are moving toward increased independent midwifery practice, which calls for maternity providers to operate in a more commercial manner (Page \& McCandish, 2006) As a means to achieving this, the application of marketing segmentation frameworks, can only benefit understanding of the drivers of pregnant health consumer behaviour.

Despite pregnancy being a common and frequently occurring human event, this particular group has received very little attention from academic marketers and marketing practitioners. It is difficult to explain why pregnancy has not been a research focus for the marketing discipline. It may be because medical dominance of health systems has tightly regulated and controlled research paradigms and/ or health goods and services provided, or perhaps because few academic marketers have found the area compelling as a research focus. This work will help to fill a substantial gap in the healthcare marketing literature by applying marketing theory (particularly healthcare marketing, social marketing and a psychographic segmentation approach) to comprehensively capture the attitudes and behaviours of the pregnancy health lifestyle group. Indeed it is worthwhile to ground this work in a marketing lifestyle paradigm, while acknowledging and incorporating the large body of knowledge that exists in the health literature. A research approach that aggregates pregnancy health lifestyle groups into holistic collectives of variables (including values, attitudes, interests, health behaviours, media preferences, motivation and perceived barriers) is a useful addition to more traditional forms of pregnancy segmentation, such as previous medical and obstetric history and demographics.

Health promotion and social marketing campaigns intended to influence health and other socially relevant behaviours, and/or shift valence or increase accessibility to attitudes and ultimately reshape health behaviours, are dependent on effective communication strategies and audience segmentation. Attention to these processes is essential in order to connect with specific needs, concerns and motivations of certain audiences (Slater \& Flora, 1991; Whyte, Keller \& Slater, 2008). Slater (1996) states that, given the health behaviour problems faced by a number of countries around the world which are urgent and costly, and the difficulty reaching many of these audiences through communication strategies, it is most important not to compromise efforts through impromptu and ad hoc 
segmentation strategies. For this reason, he recommends the continued development and refinement of sophisticated health audience segmentation tools. This study contributes to that ongoing process of development.

\subsubsection{IMPORTANCE OF THE RESEARCH FOR ACADEMICS}

The contribution and value of this proposed work applies equally to both healthcare marketing and professional or clinical health knowledge. The development of a pregnancy health lifestyle segmentation system (Gravidographics) using marketing methodologies contributes to both marketing and healthcare knowledge, by significantly broadening the concept and application of 'lifestyle' as it is currently applied to maternity care. The results of this study will contribute to advancing the segmentation methodologies currently used in pregnancy, and extend existing classifications based on demographics and/or conventional medical diagnostic criteria. Furthermore, pregnancy as a transition period in the lifecycle will be better understood in regard to its main drivers and how it is experienced by women from a health lifestyle perspective. Additionally, this study adds useful conceptual insights to The Family/Household Lifecycle Model (see for example, Bristor \& Quall, 1984)., particularly regarding consumer lifestyles in the 'spaces' (transitions) that carry a person from one lifecycle classification or life stage to another.

\subsubsection{VALUE OF THE RESEARCH FOR PRACTITIONERS}

For practitioners, pregnancy as a transition event in the lifecycle will be better understood in respect to its main drivers and how it is experienced by women. Many changes have occurred in this market over the past few years that make it a potentially lucrative market for providers of health goods and services. Marked consumer heterogeneity in lifestyle, resources and preferences constitute a wide range of opportunity for both health professionals and marketers. Much of the current theory from which practitioners inform their decisions, however, are based on assumptions that are increasingly becoming challenged against a rapidly changing social landscape (for example, same sex parenting) and increasing options for childbearing with increasingly sophisticated birth technologies.

The results of this study will also assist marketers (of both conventional and social products) and maternity service providers to design appropriate products and positioning strategies. The managerial recommendations provided highlight ways in which marketers and health providers can increase their industry's marketing orientation, meet needs well and interact with audiences effectively. 
This will assist in increased perception of quality and customer satisfaction, and will enhance other outcomes (whether measured financially, by improved social and health outcomes, or as service quality and cost effectiveness).

Social marketing practitioners are well aware of the value of understanding their audiences before designing and implementing strategies. This study will be helpful to those practitioners who have an interest in the childbearing market and an in-depth understanding of pregnant health consumers. It will do this by increasing understanding of attitudes, choice criteria and relevant saliencies that will ensure the formation of effective communication strategies for each segment. This may be primarily for information transference as a public service announcement, health promotion and maintenance communications and/or persuasion based communication strategies to target desired behaviour change.

\subsection{CHAPTER 1 SUMMARY}

In Chapter 1 an introduction was given to the context of the Transitional Health Lifestyle of Pregnancy and the rationale for a study that segments women into lifestyle segments or clusters. The knowledge can be used to better understand the market in order to design health goods and services, including tailored communications programmes. The pregnancy health lifestyle is defined as transitional and therefore embedded in knowledge pertaining to lifecycle models, consumer decision making and life stage and transition theories.

Reflecting on the main purpose of this study, the health lifestyle of pregnant women, the chief value of this study, is to contribute knowledge to healthcare marketing and social marketing in the important area of maternal health during pregnancy. The study achieves this through the application of a conceptual framework that encompasses pregnancy as a transitional and complex health event and identifies health lifestyle segments with pregnant nulliparous women.

The thesis consists of five additional chapters. Chapter 2 presents a literature review focusing on the main contribution areas. Chapter 3 discusses the conceptual framework. Chapter 4 explains the study design and operationalisation, whilst Chapter 5 presents results. Finally, Chapter 6 contains a discussion of the findings, along with limitations, managerial recommendations and suggestions for future research. Appendices and attachments follow Chapter 6. Chapter 2, the Literature Review for the study, now follows. 


\section{CHAPTER 2. LITERATURE REVIEW}

\subsection{INTRODUCTION}

This chapter examines and reviews the various literature that informs the study. It consists of several sections, each developing the study's context and relevant themes. Section 2.2 discusses the context of healthcare marketing and social marketing, as this forms the platform for the study approach, investigations and way in which findings are interpreted and applied. Section 2.3 introduces the concept of pregnancy within a consumer context, in which maternity services are consumed. Historical issues that impact on the way pregnancy may be viewed and experienced by women and practitioners today are presented. Section 2.4 discusses the pregnancy health lifestyle, based on transition theory and how consumption needs change as one moves through stages of The Household Lifecycle. Section 2.5 discusses the concept of lifestyle, psychographics and life in transition, including a review of psychographic measurement, a consideration of health lifestyles and more specifically, pregnancy health lifestyle measurement. This section also identifies the components of the pregnancy health lifestyle, physical and psycho-social, and discusses how a successful pregnancy health transition within the life course might be identified. The extent to which pregnancy health lifestyle is a trajectory of usual patterns or one where attitudes and behaviours may be changed permanently is also explored. Lastly, section 2.6, consumer decision making processes in pregnancy health, along with how health communications can be used to influence and change behaviour, are considered and discussed.

The aims of this study demand a wide understanding of numerous concepts. In order to achieve a satisfactory level of understanding and breadth, each section reports the main or seminal works in the area, followed by those conceptual or empirical works that are most relevant to the specified research aims.

\subsection{MARKETING FRAMEWORK: HEALTHCARE MARKETING AND SOCIAL MARKETING}

Whilst this thesis is essentially a lifestyle and segmentation study of a specific consumer group (pregnant women in New Zealand), the influences of healthcare marketing and social marketing form a central part of the enquiry. Healthcare marketing and social marketing perspectives are used to frame the study context, 
review findings and determine final recommendations. Women experience pregnancy while receiving health services within service offerings that can be explained by healthcare marketing. Likewise, many consumers have formed attitudes and gained pre-existing knowledge through exposure to social marketing type endeavours operating in the marketplace.

\subsubsection{HEALTHCARE MARKETING}

Healthcare marketing is the application of marketing principles to health delivery systems, and takes as a given that basic marketing concepts can be applied to the healthcare environment, in order to meet stakeholder needs (Stremersch, 2008).) Healthcare was a relative latecomer to marketing's agenda, possibly because of marketing's initial focus on profit making activity (Kotler, 1979; Stremersch, 2008).). Debate throughout the 1960's and 1970's focused on whether marketing, whose essence was the exchange of mutually satisfactory values, was applicable to both non-profit and profit based organisations (Laczniak, Michie, Donald, Levy, et al., 1979; Levy, \& Kotler, 1969;). Eventually, marketing's appropriateness in healthcare was generally accepted, mainly because of changes in areas of health, such as funding, competition and selfgovernment that support the use of more commercial strategies. In addition, the advent of healthcare consumerism led to the adoption terminology, such as 'healthcare consumer' (Stremersch, 2008). This type of language not only implies there is a marketplace in health where value-based exchange occurs, but also that a healthcare recipient might be actively involved in their care decisions.

In regard to this thesis, the application of existing healthcare marketing literature to the New Zealand context is limited, as much is written about health systems that are markedly different to New Zealand (for instance, 'Managed Care', as is typical in the United States, where much of the literature is generated). Furthermore, there is little specific literature on healthcare marketing in the area of pregnancy and childbirth.

In New Zealand, most maternity care is funded from the public funded by the government with minimal private care and a strong focus on meeting community needs. Over the past twenty years, New Zealand women have, in theory, had increased choice and control over their pregnancy health management through the advent of Lead Maternity Care, and the Midwifery Partnership Model adopted by midwifery as a guiding philosophy for relationships with clients (Freeman, Timperley \& Adair, 2004). This philosophy requires that providers operate in a 
commercial market, and that consumers are informed of service offerings and how expectations are managed and quality assured. In practice, resources are stretched, but the notion of choice remains central to present arrangements.

\subsubsection{SOCIAL MARKETING}

A more recent addition to marketing's involvement in health has been the advent of social marketing, which involves "the application of marketing concepts, tools and techniques to any social issue" (Donovan \& Henley, 2003, p.1). Social marketing has enjoyed a significant growth in both academic attention and uptake in government sector practice and funding over the past few years (Donovan \& Henley, 2003). There are numerous applications of social marketing principles to current societal problems, such as safe driving campaigns, dietary change and sun protection (Donovan \& Henley, 2003). Currently, the emphasis appears to be on health-related issues, particularly smoking and obesity, as they are the some of the most pressing for the future health of societies (Donovan \& Henley, 2003; Fuhrel-Forbis, Nadorff, \& Snyder, 2009).

Social marketing messages are primarily distributed through targeted campaigns and media (brochures and posters), but can also be heard through more mainstream forums, such as television documentaries and, increasingly, reality shows (Dutta-Bergman, 2006). There is little literature on the use of social marketing principles by health professionals in their clinical practice, although this is starting to emerge (Lilley, 2007). Social marketing and health promotion disciplines utilise health behaviour change models to explain how individuals might engage in thinking about and taking action to cease, modify or maintain certain health habits. With such understanding, marketers can devise communications strategies that will be most effective for the target audience. Social marketing draws upon behaviour change models: such as, the TransTheoretical Model of Behaviour Change (Prochaska, DiClemente, \& Norcross, 1992; Prochaska \& DiClemente, 1992, 1993), The Health Belief Model (HBM) (Janz \& Becker, 1984; Risker, 1996; Donovan, 1992), the Protection Motivation Model (Rogers, 1975; Donovan \& Henley, 2003), the Theory of Reasoned Action (TRA) (Ajzen \& Fishbein, 1980), the Theory of Planned Behaviour (TPB) (Ajzen, 1991), and more recently, the Precaution Adoption Process Model (PAPM) (Weinstein \& Sandman, 1992; Weinstein, Lyon, Sandman, \& Cuite, 1998).

The Precaution Adoption Process Model (PAPM) is relevant to this study, given its similarities with the Health Belief Model. It also, however, accommodates 
situations where a person has not become aware or engaged with a potential threat to health or safety. These situations are possible, as some aspects of pregnancy health are novel. Whereas other models assume the person is aware and responsive of a specific threat, PAPM has seven stages: stage 1, complete lack of awareness; stage 2, aware but not personally engaged; stage 3, engaged and deciding what to do; stage 4, deciding not to act; stage 5, deciding to act; stage 6, acting; and stage 7, maintenance (Weinstein \& Sandman, 1992).

While social marketing models are useful in explaining how individuals are motivated to change attitudes or behaviour accordingly, they do not examine or explain what individuals do to maximize potential for gaining and maintaining optimal health, in the absence of any problem. To explain this type of behaviour, frameworks such as the Health Promoting Lifestyle framework (HPL) (Walker, Sechrist \& Pender, 1987) have significant utility, as they categorise the various dimensions of a person's health.

Some authors attempt to combine models that originate from different perspectives. For instance, Risker, (1996) combined the consumer decision model with The Health Belief Model, proposing this as a more accurate analysis of consumer health services purchasing and one that would lead to increased understanding of consumer information search behaviour in health. Donovan and Henley (2003, suggest model choice can be complicated in situations where no one model is completely suitable and that it would be useful to combine differing aspects from several models. The authors advocate a pragmatic or eclectic approach, whereby synthesizing models can more comprehensively address an issue.

Another problem with the wholesale application of any of the change models such as the Health Belief Model is highlighted by Hagoel, Ore, Neter, Silamn, and Rennert (2002), who believe that by focusing on cognitive elements in predicting health behaviours, studies risk focusing on one health problem at a time. This causes a phenomenon to be regarded as distinct and discreet, as opposed to existing within a lifestyle. Chin, Monroe, and Fiscella (2000), claim that because medicine traditionally relies on a rational choice model as a vehicle for promoting health behaviour, it has overlooked social class and health behaviour relationships. Therefore, according to Chin et. al, the use of behavioural change models (for instance, Theory of Reasoned Action, Trans-Theoretical Model and Health Belief Model), based on the perception of the health consumer as rational 
and goal directed, is flawed. They conclude that models that trumpet the ability of the individual to choose, and focus on individual behaviour alone, fail to understand social structures and contexts that can promote unhealthy behaviour.

It would be fair to say that pregnancy has not received any significant attention from social marketers to date, or formed the basis of contributions to the academic literature. For the pregnant consumer, government generated brochures and printed materials provide information to the mainstream (albeit with some ethnic targeting). Issues such as folic acid supplementation, smoking in pregnancy, avoidance of alcohol and unsafe foods, and breastfeeding present the most significant activity in the area. Moreover, these materials are written from an informational perspective and are distributed through healthcare practices, maternity carers and antenatal education organisations. Pregnant women and their partners also have access to various antenatal education programmes offered by childbirth educators. The Internet provides an additional rich arena of both quality and non-quality assured pregnancy health information.

There are potentially some definitional boundaries between social marketing and health promotion used by health specific disciplines. For the purposes of this study, it is recognised that both these applications contribute and have utility in targeting consumers to alter identified non-health promoting behaviours around pregnancy in this multi-disciplinary area.

The singular and combined efforts of healthcare marketing and social marketing contribute to the building of relationships with consumers. These marketing relationships manifest in the one-on-one relationship with healthcare providers. An in depth understanding of consumer behaviour, along with effective segmentation and targeting strategies, contributes significantly to the establishment of these relationships.

\subsubsection{SECTION SUMMARY}

This section of the chapter has introduced health care marketing and social marketing as important background to how this study is positioned within the wider marketing area. Health care marketing is important to the way health providers offer services and build relationships with their clients. Social marketing provides frameworks and models, and is another way in which health behaviours can be understood and modified in order to achieve better health 
outcomes. The next section of the literature review discusses pregnancy within a consumer context.

\subsection{THE CONTEXT OF PREGNANCY}

\subsubsection{INTRODUCTION}

This section examines pregnancy as an event in the life course of women in consumption settings. In comparison to earlier times, pregnant women today are consumers with more autonomy; they have increasing control over decisions and choices that are important to themselves and their babies.

Firstly, key historic factors are explained that are relevant to how pregnancy is conceived within today's marketplace. The section concludes with a discussion of the structure through which maternity care is provided in New Zealand.

The period of a woman's first pregnancy involves an altered state of being, lasting approximately forty weeks. There are considerable physical changes that involve accommodating and supporting the developing baby. There is also an important psycho-social dimension that requires accommodation of a transition that marks the passage between childlessness and motherhood (Mercer, 2004). Pregnancy straddles the period from conception (or perhaps from some consumers' perspective, from a positive diagnosis of pregnancy) to birth. Arguably, an understanding of pregnancy health lifestyle could also include the pre-pregnancy planning period, especially where consumption (be it health services, consumer products or adjustments to health behaviour) occurs in anticipation of an altered health state (Green-Raleigh, Lawrence, Chen, Divine \& Prue, 2005)

Although the majority of Western women today will experience this event relatively infrequently in their lifetime (1-3 times on average), pregnancy is a central and constant part of the community and social landscape. The desired outcome, a healthy mother and baby, is critical to the overall wellbeing and progress of populations. Therefore, much of a woman's potential behaviour is influenced and even critiqued by higher level factors, such as social, cultural and religious norms and taboos (Liamputtong, Halliday, Warren, Watson, \& Bell, 2003 Ayaz and Efe, 2008). Over time, pregnancy and childbirth has also been subjected to political forces. This subjection has directly impacted on how pregnancy is viewed within society and the ways that maternity services have consequently been designed (Belgrave, 1991). 


\subsubsection{HISTORICAL INFLUENCES}

Pregnancy and birth in New Zealand are highly political and several factors have significant influence over the environment in which women form attitudes and beliefs. Changes in practice have shaped obstetric care and consequently consumer attitudes and behaviour for the past hundred years; for instance, legislation covering maternity service provision and the tensions between medicalisation of childbirth versus pregnancy as a natural event. These changes are discussed below.

In New Zealand, a successful redefinition of childbirth by doctors, from a natural event to a pathological process in the early 1900's, resulted in the medicalisation of childbirth. Death during childbirth was an ever present threat and major fear for women up to the early $20^{\text {th }}$ century (Loudon, 2000). The medical profession proposed that maternal mortality and morbidity statistics could be improved, if the control of obstetrics was under the authority of the medical profession; thus, the birth was moved from the home to the hospital and under the care of medical practitioners (Belgrave, 1991). Likewise, the location of maternity care exchanges also changed. The production and consumption of maternity services that had previously occurred in the home, was now provided by hospitals (Stojanovic, 2008). Despite the risk of maternal death in childbirth being now less significant in the developed world, it is still an underlying concern for women during pregnancy (Szeverenyi, Poka, Hetey, \& Torok, 1998; Hofberg \& Ward, 2003). Until recently, the patient-provider relationship was characterised by a lack of autonomy and power for the woman. A tension between doctors and midwives in New Zealand continues today (Haines, 2009), and remains an influence over women's attitudes toward, and experiences of, the health service during pregnancy.

The question of whether pregnancy and childbirth is a natural or pathological event impacts substantially on a woman's perception of her health in pregnancy, and thus, her resulting behaviour. For example, in Britain, legislation for pregnancy is framed within disability and sickness: The Pregnancy Discrimination Act (1978) and Family and Medical Leave Act (1993) (Tulman \& Fawcett, 2003). Women's health in pregnancy has been studied primarily in terms of occurrences of illness or complications (Cunningham et al., 1997; Tulman \& Fawcett, 2003). Many textbooks teach 'recovery' from childbirth, implying that pregnancy is an illness (Tulman \& Fawcett, 2003). If pregnancy is viewed as an event that 
requires close surveillance by the medical profession, this influences the level of choice, autonomy and decision making women are likely to have.

Other historical factors continue to contribute to how pregnancy is perceived. Victorian beliefs and behaviour codes regarded the physiology of childbearing as a private female matter (Flanders, 2003). Public discussion of practices, or activities and consumption associated with childbearing, was often avoided and discouraged. This led to the formation of a situation where membership and consumption of related products was largely private and discreet. Indeed the term 'confinement', used until the third quarter of the $20^{\text {th }}$ century, related to the withdrawing from society expected of women (Flanders, 2003). Market knowledge and access was available only to those who were considered legitimate. Although attitudes toward pregnancy and child rearing matters legitimately occupying a 'public' space have loosened markedly in current times, arguably there are still sensitivities around the behaviour expected of women in this life stage.

\subsubsection{THE NEW ZEALAND MATERNITY CARE SYSTEM}

In New Zealand, maternity services have undergone significant change over the past twenty years (Haines, 2009). Changes to The Nurses Act in 1990 saw the passing of law that enabled midwives to practice independent of a doctor (Guilliland, 1999) and then on to a system of Lead Maternity Carer (LMC), the main health professional designated for women through the childbearing experience. This period has also been evolutionary in terms of service design and configuration for maternity services. Over successive years, this has led to almost all general medical practitioners exiting from primary birth provision. This change corresponded to women, who would normally attend a doctor for pregnancy care, moving to the care of midwives. Many midwives now choose to practice not only independently of a doctor, but mainly outside of hospital employment (Guilliland, 1999).

Furthermore, until the early 1990's, New Zealand maternity services were structured and delivered within a medical model, similar to other parts of the health service. Midwives were also Registered Nurses, who worked under the supervision of a medical practitioner. Prior to 1991, primary obstetric services were provided by a woman's medical practitioner or obstetric specialist referred by her doctor. Midwifery contact occurred in hospitals, during antenatal education classes, inpatient labour support and postnatal care. There was an 
active but small homebirth movement, but this also relied on the inclusion and direction of a consenting medical practitioner in each case. Consumerism, feminism, and opposition to the medicalisation of childbirth by professional and consumer lobby groups, have had significant influence over attitudes and the way providers are now perceived (Donley, 1986; Belgrave, 1991). For the vast majority of New Zealand women, midwives provide total maternity care, based on the predominant 'Midwifery Partnership Model', which identifies how power can be shared without the need for equality (Freeman et al., 2004).

Today $70 \%$ of primary (low risk) women choose a midwife as their Lead Maternity Carer (Leap \& Barclay, 2001; Ministry of Health, 2004). Other options for care available for women include general practitioners, private obstetricians, and District Health Board (DHB) primary and high risk care. All of these methods involve contact with a midwife in some capacity, whether as the LMC, in a shared care arrangement, or within hospital provided care. High risk women are generally cared for by a medical specialist in obstetrics. In addition, providers have themselves become more diverse. The midwifery profession consists of a collective of practitioners, who individually may have very divergent philosophies of care as expressed by variation in preferred modes of practice (Haines, 2009).

Furthermore, regional differences exist as to the type and accessibility of LMCs available. Although there are some problems in the system, (shortages of midwives in some centres, media attention on the quality of midwifery care), this system is the main system of choice and most likely to continue for the foreseeable future (Freeman et al., 2004).

\subsubsection{THE MATERNITY CONSUMER}

The makeup of the pregnancy market has become substantially more heterogeneous when compared to first time motherhood in the preceding decades (Grindstaff, 1996; Hewlett, 2002; Fetto, 2003; Page \& McCandish, 2006), thus making the notion of segmentation very feasible. Even the assumption within Western societies that 'women have babies' is now contestable, with increasing numbers of women within the childbearing bracket opting out (Berryman, 1991; Malson \& Swann, 2003). Several changes have occurred that have altered the composition of this group and these are discussed below.

Firstly, there is now significant diversity in maternal age of first birth, (particularly in regard to extremes of age, from teenage pregnancy to a 'greying' of the 
market). The average maternal age for a first child in New Zealand is steadily increasing (Maternity Report, 2004). It is not unusual for commencement of childbearing to be delayed until the third decade of life. This has subsequent effects on transit into and consumption behaviour in other lifecycle stages (Schnaninger \& Lee, 2002).

With modification of family structures (multiple and dynamic household forms), and cohort variation there is a large variety of childbearing households. For example, a childbearing woman may be a member of the 'baby boomers', 'Generation X' or the 'Echo Boom' cohort. The 'Net Generation' comprising those born between 1977 and 1997 and 'Generation $Y^{\prime}$ ' have now reached or are approaching childbearing age and may have quite different attitudes toward childbearing. The 'Net Generation' is the first generation to grow up with computers (Tapscott, 1998) and may have more positive attitudes toward the use of technology in pregnancy and childbirth than previous cohorts, as found by Zechmeister (2001) and Green and Baston (2007). Within the Western World, this presents a potential conflict with the preceding generation of childbearing women, who campaigned vigorously for natural childbirth, including rejection of medicalisation and technological interference (Stojanovic, 2008).

In New Zealand, several ethnic minorities are increasing in the percentage of the population they make up; in particular, Māori, Pacific Island and Asian populations. The Māori population is considered young, with $42 \%$ of Māori currently aged 17 or under (Te Puni Kokiri, 2000, p.13). Resurgence in Maoridom has led to re-enculturation of many traditional practices into the lifestyle of some Māori (Krisjanous \& Love, 2002), leading to a demand for processes and procedures that are sympathetic to ethnic childbirth organisations such as Nga Maia, a national body representing Māori birthing. The increase in immigration from Asian countries and the ongoing growth of Pacific populations in New Zealand also leads to a 'browning' of the New Zealand ethnic mix. Thus, a significant percentage of the childbearing population undergoes acculturation into a new culture.

Assisted fertility is a major influence in the market and allows for substantial variation in the pathway to pregnancy. This in turn adds to a greater diversity of consumer behaviour and lifestyles around the period of conception, and possibility the whole of pregnancy, birth and the post partum. Hirschman (1991) suggests that the emergence of novel reproductive markets, such as surrogacy and in-vitro 
fertilisation, mix aspects of sacred and profane marketing exchange that historically in most cultures have been taboo. Increasingly, women, who would have previously been unable to conceive or sustain a viable pregnancy, are able to become pregnant and expect a live birth.

\subsubsection{PREGNANCY AS A DRIVER OF CONSUMER DEMAND}

From a marketing perspective, there is an increasing proliferation of goods and services aimed at meeting consumer needs that will support a pregnancy lifestyle: for example, branded maternity clothing, pregnancy diagnosis kits, creams and toiletries, books and magazines and stem cell banks.

In addition, the effects of the reorganisation of many traditional maternity care systems into new modes of delivery (such as midwife only care) over recent years are now evident within contemporary maternity consumer markets (Leap \& Barclay, 2001; Ministry of Health, 2004). The loss of more directive traditional systems has brought about a need for marked changes in maternity consumer behaviour, especially in terms of information search and purchase patterns. It is only in the past two decades, since the advent of independent midwifery practice, that pregnant consumers have been required to participate in the choice of an LMC. In addition, little is known from a marketing perspective about desired provider attributes and the corresponding consumer decision making process engaged in by pregnant women.

To adapt to market changes, the emergence of greater consumer participation and increased consumer access to information (for example, via Internet information and social networking sites) can be observed more generally. Social media is rapidly increasing the interconnectivity of like consumers, allowing rapid and widespread communication of information, attitudes and experiences about health (Lewis, 2006; Lemire, Paré, Sicotte, \& Harvey, 2008). The changing roles of the midwifery and medical professions have changed the dynamics between provider and consumer, with consumer attitudes such as health consumerism becoming more evident. With midwifery also advocating a partnership model with women, this potentially places a higher demand on women to be active information seekers, decision makers and participants (Freeman et al., 2004).

Furthermore, while safety of the mother and baby is still paramount, a relatively reduced risk of morbidity and mortality has led to a higher level of consumer needs and expectations being met; for example, seeking a 'meaningful 
experience' is a somewhat vaguer goal compared to the tangible and measurable outcome of avoiding maternal or infant death in childbirth. Regardless, the former is now very important to many maternity consumers (Halliday, 2000; Halliday \& Hogarth-Scott, 2000). To this end, many goods and services available in the pregnancy marketplace now rely on consumer satisfaction and fulfilment as key outcomes, particularly in competitive markets.

A feature of pregnancy as a health state is that the developing fetus is dependent on the choices and behaviours made by a host person. This makes the underlying motivations and obligations of the pregnant woman unique in regard to consumption activities. For most women, 'wanting the best for baby' is a powerful influence on any decision or choice made in relation to their pregnancy (Freeman et al., 2004).

\subsubsection{SECTION SUMMARY}

This section has discussed the background of pregnancy in New Zealand, and the historic influences that have shaped opinions and confidences about where pregnancy sits in the medical/midwifery paradigms. This is important because it influences women's attitudes and beliefs about themselves and the meaning of pregnancy. Pregnant women, as a consumer group, have changed markedly over the past decades. There is significant diversity of consumer demographics and types of family structure. In addition, consumers' involvement and responsibilities have shifted as a predominantly paternalistic service design has evolved into new models, in particular, the Midwifery Partnership Model.

The next section of the Literature Review discusses pregnancy as a life stage transition.

\subsection{PREGNANCY AND LIFE STAGE TRANSITION}

\subsubsection{INTRODUCTION}

This section of the literature review provides a backdrop to the pregnancy health lifestyle, based on transition theory and how consumption needs change as one moves through stages of The Household Lifecycle. Pregnancy is an event that occurs within the lifecycle of individuals and families. The period of expectation of a first baby leads to significant planning and anticipation, and can be viewed as a significant transitional period between one life stage to another. 


\subsubsection{HOUSEHOLD LIFECYCLE MODELS}

Pregnancy occurs during a transition between stages of the Household Lifecycle Model (HLC) (Young Married to Full Nest 1). HLCs (historically referred to as Family Lifecycle Models) are used in many disciplines, including marketing, economics, sociology and family sciences, to explain consumption at different stages. The HLC applies in this context and helps understand reasons behind consumption and lifestyle changes that result from transitions in the life course, such as pregnancy.

Schaninger and Danko (1993) state researchers have "long been enamoured of the family lifecycle concept because it captures lifestyle, income and expenditure pattern differences brought about by family role transitions" (p.590). Household Lifecycle Models are useful for marketing segmentation because they mark stages or transitions associated with distinctive changes in expenditure for goods and services (Wells \& Gubar, 1966; McLeod \& Ellis, 1983 Wagner \& Hanna, 1983; Schaninger \& Danko, 1993; Wilkes, 1995). With the above factors in mind, and as a way of linking pregnancy to the lifecycle, this section provides a general discussion on Household Lifecycle Models, including their development and utility in explaining transition within the life course.

Early articles, such as Ando and Modigliani (1963), based on an economic lifecycle hypothesis (originally proposed by Rowntree, 1903), became the foundational model for consumption and saving behaviours. Some of the earliest family lifecycle models were proposed by Duvall (1955) and Wells and Gubar (1966). Lansing and Morgan (1955) introduced the lifecycle concept to marketing and incorporated the terms, 'Full nest', 'Empty nest' and 'Solitary survivor'. Wells and Gubar's (1966) Family Lifecycle Model became the traditional model most commonly adopted by marketers. This model included five stages of families, followed over a lifetime: young singles, young married, parenthood (full nest), post-parenthood (empty nest) and dissolution. Reflective of the times, it excluded most non-family and non-traditional households. Traditional models such as Wells and Gubar (1966) were developed during the post World War Two period, when there were high birth rates, low divorce rates, and the nuclear family was considered the norm, if not the ideal. Transition through stages in the traditional models was consecutive, with no recognition that some may 'recycle' to former stages. 
By the late 1970's, The Wells and Gubar (1966) model came under criticism because it considered only households that were traditional and followed a normal pattern of progress through the lifecycle (Gilly \& Ennis, 1982). Western society was rapidly changing, with non-traditional households becoming increasingly common (McLeod \& Ellis, 1983; Wagner \& Hanna, 1983; Schaninger \& Danko, 1993; Wilkes, 1995). Work was undertaken to modernise the family lifecycle by capturing previously excluded arrangements and better reflecting backward or recycled flows.

Increasingly, transition between stages is neither clear cut nor consistent across individuals or households (Schaninger \& Danko, 1993). The most widely referenced modernised family lifecycles are those of Gilly and Ennis (1982), Murphy and Staples (1979) and Wilkes (1995) that attempt to capture more fluid household arrangements and structures. A recent significant modification to the full nest household stages has been the separation of traditional full nest couples from those who delay marriage and childrearing until their third decade, due to the finding of different attitudinal and consumption profiles across these respective groups (Schaninger $\&$ Lee, 2002). With pregnancy as a time or event that marks transition from 'Young Couple' into 'Full Nest 1' (as in Wilkes), this period is well worthy of research focus.

There is now general agreement that these models have to deal with increasing complexity due to the variety of ways people enter and exit stages of the lifecycle, including Full Nest 1 (households where members are parenting at least one child under the age of six; Wilkes, 1995). As stage boundaries are becoming less well defined, and as modernised HLC models attempt to address new stage definitions, it stands to reason that the transitional periods are likely to become more important than the link spanning two discreet stages.

\subsubsection{TRANSITION THEORY AND TRANSITION INTO MOTHERHOOD}

Life stage transitions (which may be brought about by intentional forward movement or by unexpected and unplanned events) have been described as times of personal changes in attitudes and practices, as a result of changes in social networks, health concerns or resources (Borgatta, Bulcroft, Montgomery \& Bulcroft, 1990; Devine, Bove \& Olson, 2000).

The extent to which a person's lifestyle changes over time will be influenced by many things, including material changes due to alterations in resources, status, 
or health. Transitional lifestyles are time bound and linear, meaning they function as a bridge from one life stage into another. As well as pregnancy, other transitional lifestyles that could be considered include marital separation, redundancy, dynamic nest (grown children who come and go) and households with a terminally ill member. 'Synchographics', a relatively recent marketing approach, is a marketing tool used to predict and target 'households in motion' (Goss, 1995) where changes (such as birth) bring about the need for purchase and consumption of products to support changes in life circumstances and corresponding lifestyle.

Transition theory offers an explanation of the processes a person undergoes during adaptation to a new life stage. Whilst the marketing literature pays little attention to transition, the health literature, particularly nursing theory, views transition as a concept central to health (Schumacher \& Meleis, 1994) and is relevant to this work. Within the health literature, Newman's (1986, 1997 'Theory of Health as Expanding Consciousness' describes 'transcendence' (acceptance of an unchangeable situation), and 'life re-orientation and transformation' as processes that occur during life events or changes, particularly in regard to health. Kralik, Visentin and van Loon (2006) provide a comprehensive review of how the term 'transition' has been used in the health literature. They note that transition theory has a long history in other disciplines, particularly in marking rites of passage where stages of human life are marked by socio-cultural rituals (p. 322). They conclude that transition is an important concept which marks movement and passage, and "always beginning with an end" (p. 325). Schumacher and Meleis (1994) state the universal properties of transition are process, direction and change in fundamental life patterns. Conditions that may influence the experience and quality of outcomes are meanings, expectations, level of knowledge and skill, environment, level of planning and physical wellbeing. Indications of a successful transition are subjective well-being, role mastery and the well-being of relationships (Schumacher \& Meleis, 1994). Schumacher and Meleis also offer that at the individual and family level, changes occurring in identities, roles, relationships, abilities and patterns of behaviour constitute transitions. Price (2003) studied professional women's retirement adjustment within the frameworks of identity theory and role theory and as one of the many discontinuities women experience during their lives. They found four stages of transitional growth could be identified during the transition process. A study of first time fathers by Condon, Boyce and Corkindale (2003) examined gender specific factors for psychological 
distress during the transition to parenthood amongst first time fathers and found that the pregnancy period, rather than postnatal, was most stressful. Finally, McDermott, Dobson and Russell (2004) examined changes in smoking behaviour among young women over four life stages and showed that adoption, maintenance and cessation of smoking among young women are strongly related to major life stage transitions.

Within the marketing literature, Lastovicka, Joachimstaller, Erich, Bhalla and Scheurich (1987) studied young male drink drivers, describing how many drivers belonged to a risky driving group while young, but eventually move into another grouping of more responsible drivers ('well-adjusted group') or remain arrested in the 'good timer' group.

Life course and life stage trajectories are a focus for researchers interested in how consumers or persons adjust to changes that need to be made to meet new life demands, and pregnancy can be seen as a distinct trajectory within a woman's life course. As the past provides the basis for experience of current events, the life course perspective includes concepts of continuity and change across the lifespan in a historical and social context (Mead, 1938; Elder, 1987; Devine et al., 2000). Life course trajectories therefore represent a pathway through life; the accumulation of thoughts, attitudes, beliefs and actions as one approaches life transitions (Devine et al., 2000). Devine (2005) and Paquette and Devine (2000) report studies of trajectories in regard to food choices, and define trajectories in this context as "persistent thoughts, feeling, strategies and actions with food and eating developed over the life course" (Devine, 2005, p. 568). Devine lists four keys features regarding behavioural trajectories: first, persistence and stability, with minor adjustments and few turning points; second, the inclusion of thoughts, feelings and strategies; third, accumulation and development over a lifetime, incorporating meaningful experiences; and fourth, trajectories reflect individual development in context, reflecting social and historical changes and availability of resources, and being influenced by where people live their lives. Changes such as life events, change in residence and role shifts often bring about adjustments overtime within existing trajectories (Devine, Connors, Bisogni, \& Sobal, 1998).

Generally the trajectories, in which these adjustments occur, are persistent and embedded in a social and cultural context, with relatively few major permanent changes (Devine, 2005; Paquette \& Devine, 2000). Devine (2005) discusses 
adjustments in food choice over the life course; for example, eating more fish when children leave home. Minor adjustments, such as changing the type of milk consumed, can be relatively easily accommodated and do not demand a major change in lifestyle or self-identity. Turning points in behavioural trajectories occur after drastic life events; for example, life-threatening illness of a spouse (Devine, 2005). Turning points require the reformation of personal identities, causing them to think differently about themselves, with no turning back. Devine (2005) found a relatively low incidence of turning points, occurring in peoples' lives, that influenced major dietary changes.

The pregnancy health lifestyle for the purpose of this study is posited as transitional. Adjustments to lifestyle required at the onset and during pregnancy are likely to be made within the existing trajectory or pathway of a woman's health framework. However, a 'turning point experience' could occur, such as the onset of gestational diabetes that highlights the necessity of change in order to protect from a future health threat, particularly maturity onset diabetes later in life; albeit, such events occur in the minority of women.

The transition to first time motherhood is a significant event in the lifecycle of most women. Availability of information, increased choice through birth control, expectation of a live mother and baby post-partum, less births per woman and a reduction in infant mortality, have led to women regarding pregnancy as a distinct and memorable life event, over which some control is possible. Pregnancy is recognised as a period of evolving social, psychological, behavioural and biological change for the woman that is accumulative in preparation for the approaching reality of motherhood (Devine et al., 2000). New behaviours and changes are also marked by a degree of departure from present lifestyle; for instance, reducing work hours or preparing to cease work, reducing physical activity, modifying leisure pursuits due to tiredness, and having increased interest in meeting and socialising with other families with babies. Höjeberg (1985), discusses how historically lay traditions of pregnancy focus on teaching and warning a woman, helping her to leave her previous life behind, and providing for a new life as a mother. At key points, consumption of goods and services (both health and commercial) occur in order to maintain and assure health. For instance, the engagement of a maternity provider occurs most often within the first trimester and almost certainly by twenty weeks gestation. This is eventually a service provider choice decision on the part of the mother as consumer. 
Although 'pregnancy' starts at conception, traditionally it is a medical person who legitimises the beginning point, by awarding the woman a positive diagnosis of pregnancy. For most women, acknowledgment from an 'authoritative' source (that is, scientific diagnosis) is still considered a key transition marker. The availability of self diagnosis pregnancy kits has now, however, brought the ability to confirm or disconfirm pregnancy to the women themselves with relative ease, and this may well lead to a change in the way women define the beginning of a pregnancy.

Pregnancy transition means moving from inherited family to construction of one's own family, whether by nature, technology or some other means of creation of generational kinship. Those in a pre-pregnant contemplation state may engage in behaviour modifications concerned with pregnancy; for instance, smoking cessation, or the purchase of baby products in anticipation of a pregnancy. Some focus on and prepare for pregnancy for several years, such as those who experience technology aided conceptions (IVF, surrogacy, post-mortem harvesting) and those who vary greatly from the pathway of a natural conception. Therefore, the pregnant lifestyle should incorporate any period where a prepregnant lifestyle has changed to accommodate an anticipated pregnancy. In contrast, an unplanned or unexpected pregnancy that results in a late awareness may offer a much shortened opportunity to transit. Family or state adoption has always been a legitimate pathway to kin or family creation and although there is no physiological gestation period per se, it is not usually without a transitory or preparatory stage. This stage might be considered part of a legitimate prenatal period where unique consumer behaviours and characteristics can be observed.

Rubin $(1967,1975,1984)$ conceptualised the psycho-social work of pregnancy as 'Maternal Role Attainment' (MRA), made up of a matrix of tasks of pregnancy: including, 'ensuring safe passage for self and baby', 'seeking acceptance of and support for self and baby', 'binding into the infant' and 'giving of self'. For example, in seeking safe passage, acceptance of the developing fetus as a dependent being may lead to an increased recognition of responsibility for health. Alterations to health behaviour may be made solely on behalf of the baby that may not be made otherwise. An example of this is cessation of smoking for the pregnancy and resumption once the baby is born. Successful achievement of Rubin's 'tasks' by a woman demonstrate a positive transition into motherhood. 
Mercer (2004) redefined Rubin's 'Maternal Role Attainment' (MRA) with 'Becoming a Mother' (BAM). Mercer (2004) proposes BAM is a more accurate definition of the psychological transition that occurs; whereby movement from a known current reality of self moves to an unknown, new reality (p. 226). Mercer suggests BAM more aptly reflects the process as one that commences at pregnancy and continuing post-partum. The process means that learning and experiencing the role of motherhood is not just one of initial transformation but of continuing growth. This viewpoint extends the notion of transition continuing well into the onset of Full Nest I, the beginning parenting stage present in all Household Lifecycle Models.

The idea of growth as the 'work of pregnancy' is also discussed by Barba and Selder (1995), Bondas and Erikson (2001), and Mercer (2004). For instance, Bondas and Erikson (2001) put forward the outcomes of pregnancy 'work' as 'the perfect baby', 'achieving an altered mode of living' and 'family communion'.

The above paragraphs have reinforced the perspective of the pregnancy health lifestyle as a transition that requires adaptation and adjustment to successfully deal with the physical and in particular, psycho-social demands of pregnancy. The transitional health lifestyle perspective is a central part of this study.

\subsubsection{SECTION SUMMARY}

This section placed pregnancy as a life event that for most is a necessary experience to move into Full Nest 1 within the Household Lifecycle. Consumer behaviours are expressed within a collective or pattern of behaviours that make up a lifestyle. This study is concerned with lifestyle in the context of pregnancy health and transition.

As the first pregnancy experience is novel, adjustments are made to respond to a changing internal and external environment. These adjustments are most likely to occur within an existing trajectory of pattern. However, for some, a turning point experience may occur when permanent changes are made 


\subsection{HEALTH AND HEALTH LIFESTYLE}

\subsubsection{INTRODUCTION}

This section first explores the concept of lifestyle, particularly as it is expressed through values and activities, interest and opinions (AIOs). This then leads into discussion of how lifestyle can be measured through psychographics, and the issues and difficulties inherent in this. The psychographic approach used in this study is then explained. The second part of this section examines health and health lifestyle before proposing why pregnancy health lifestyle can be studied from within a psychographic frame. This part includes a brief discussion on consumer decision making processes. Understanding how consumers make decisions about lifestyle related consumption and choices to meet lifestyle goals is essential to understanding how a certain health lifestyle is adopted in pregnancy.

\subsubsection{LIFESTYLE}

Interest in lifestyle analysis and early conceptualisation of lifestyle can be traced back to Veblen's 'Theory of the Leisure Class' and his analysis of conspicuous consumption (Veblen, 1979/1899) and the work of Weber (1905; cited in Todd \& Lawson, 2001, p.270) and Adler (1927). Adler, termed the phrase 'style of life' referring to the goals people have and the means by which they achieve them Adler (1927). Bourdieu (1984, 1990 \& 1999) suggested a socio-cultural lifestyle theory. Important to his concept of lifestyle were the struggles between class and the resultant power relationships played out in the various lifestyle practices. Bordieu's (1984) concept of 'habitus' uses social class as the main classification scheme for explaining how people perceive and treat their bodies.

Lazer (1963) was one of the first consumer behaviour researchers to define lifestyle as it relates to marketing. The lifestyle concept applied to marketing is concerned with an individual's behaviour patterns that support goals of achieving the desired self image (Solomon, Bamossy, \& Askegaard, 2002, p.504) and "reflected by different ways of living and the patterns of consumption that tend to accompany them" (Todd, Lawson \& Faris, 1998, p.31). That is, the ways in which people live and spend their time and money (Kaynak \& Kara, 2001). Chaney (1996) discusses "patterns of action that differentiate people and in so doing, this makes sense of what people do and why they do it, and what doing it means to them and others" (p. 4). 
Marketing's interest in lifestyle has historically been in the domain of material lifestyle, whereby motivations along with lifestyle constraints and/or choices give rise to the pursuit of certain products and services to support one's lifestyle (Cockerham, 2005). With an increased focus on health marketing and social marketing, the concept of health lifestyle is becoming increasingly relevant (Cockerham, 2005). These two marketing sub disciplines also provide a key background in which lifestyle is explored in this study. The concept of lifestyle measurement is discussed in the next section. Following this, health lifestyle is discussed in detail in Section 2.5.4.

\subsubsection{MEASURING LIFESTYLE}

Segmentation of a consumer market, first proposed by Smith (1956), consists of the breaking down of a larger heterogeneous group and aggregation into smaller, more homogenous clusters. The need for segmentation is based on the premise that if marketers maximize profits through product differentiation, then tailoring marketing and communication efforts to different identifiable groups would be advantageous (Smith, 1956). Market segmentation draws on the conceptual development within social sciences and philosophy over the past seventy-five years, as it emerged against the phenomena of industrial society, where totalitarianism and theories of mass society and crowd psychology were paramount (Slater, 1996).

Today, consumer lifestyle is a well accepted basis for segmentation in marketing (Wells (ed), 1974, Wells, 1975; Sorce, Tyler, \& Loomis, 1989; Mitchell, 1994; Todd et al., 1998); lifestyle in turn is a reliable predictor of consumer demand and activity. Goods and services aspired to and/or purchased are able to be linked to specific lifestyle segments. Lifestyle analysis provides a comprehensive view of behaviour and the motives underlying the purchase of many goods (Todd et al., 1998). With pregnancy health lifestyle segmentation contingent upon an effective system by which to evaluate it, an applicable process is health psychographics. This process originates from earlier psychographic/lifestyle measurement techniques. The next section discusses psychographics as a basis for measuring lifestyle. 


\subsubsection{Psychographics}

This section begins with a summary of the main features of psychographics, before proceeding to a discussion of health psychographics, which is the central approach adopted in this thesis.

The term psychographics was first used by Demby (1974) who combined 'psychology' and 'demographics' in order to put "more flesh onto demographic bones to add the richness of the social and behavioural sciences to demographics" (Vyncke, 2002, p. 447). An early work in contemporary analysis, "Lifestyle and Psychographics" (Wells, 1974) brought several authors together to report their research and further develop the field. Bruwer, Li and Reid (2002) state that "segmentation should be based on identifying consumers with similar behaviour, not necessarily demographic characteristics, so that segments are more actionable from a marketing strategy standpoint" (p. 221).

The most generally accepted view of psychographics is seen as the operationalisation of the lifestyle construct (Bushman, 1982). Grouping consumers into lifestyle classifications is based on the premise that groupings of like consumers share similar attitudes, interests and opinions (AIO) personality traits and/or values (Bushman, 1982; Shufeldt, Oates, \& Vaught, 1998; Solomon, et al., 2002). Individual lifestyle classifications within a schema are comprised of homogenous groups of consumers, which can be targeted for certain products. The term lifestyle can be related to usage of product constellations, clusters of complementary products, specific brands, or more generally, a range of consumption activities. Heath (1995) describes psychographics as linking consumers' "measurable demographic characteristics with the more abstract aspects of attitudes, opinions and interests" (p. 75). Heath suggests five psychographic approaches: lifestyle profiles (demographics, product/media use and psychographic elements), product specific psychographic profiles (consumers profiles on product-relevant dimensions-dependability, styling), personality traits as descriptors (for example, concern for the environment analysed against personality traits such as tolerance and extroversion), general lifestyle segmentation (respondents' answers to questionnaires are grouped in homogeneous groupings), and finally, product specific segmentation (users in a given product category that can be grouped). In this study, a general lifestyle segmentation approach is applicable because it allows for a holistic approach to studying health during pregnancy. 
Vyncke (2002) suggests that there has been 'waves of research' within the field of psychographics. The first research 'wave' was based on personality profiles, while the second moved from personality traits to the lifestyle construct. Within lifestyle, according to Vyncke, there have been two main approaches: firstly, the Activity, Interest and Opinion (AIO) approach; and secondly and more recently, the values approach. Vyncke (2002) believes that the values approach is replacing AIO inventories, as the latter has been found to be dependent on "extensive and burdensome AIO inventories" (p. 448). Other authors suggested other approaches that may be used. Brunso, Scholderer and Grunert (2004), suggest the means ends approach, albeit perhaps an extension of the values approaches. Combination approaches have also been proposed as a means of addressing the need to obtain a mix of dimensions (Gunter \& Furnham, 1992; Wedel \& Kamakura, 2000; Vyncke, 2002; Kamineni, 2005).

For the purposes of this study, a combination approach is best, as it allows for consideration of differing dimensions that underpin a person's health. Empirical studies of lifestyle most usually involve an extensive survey an appropriate quantitative analysis. Psychographic statements can either be standardised, or ad hoc, with choice dependent on objectives of the study (Tam \& Tai, 1998). One or a combination of both can also be used (Wells, 1975).

One of the best known commercially used lifestyle typologies based on values, is the SRI VALS (values and lifestyles) typology. Created first in 1978 by The International Stanford Research Institute (SRI), it was revised in 1989 to render VALS 2, with an emphasis on two dimensions, self orientation and resources, and nine general psychographic segments overall. Each classification contains a rich description of the occupants, who would fit the profile. Classifications have a strong link to demographics. Studies informed by the VALS2 technique have been conducted in New Zealand (Muellar-Heumann \& Fiegler, 1979; Lawson, Rummel, Fiegler, \& Mueller-Heumann, 1990; Lawson \& Todd, 2002), where similar parallels to VALS 2 segments have been found. Studies of the New Zealand population using the SRI VALS, methodology such as Lawson and Todd (2002) are important, as they affirm the value of a strong methodological heritage, as well as providing a more tailor made psychographic typology of New Zealanders.

Today, psychographic lifestyle studies usually include attitude, values, AIO, demographics, and media use. Typically, the first three dimensions are used to 
group people, with the others used to provide a fuller description or profile of each group (Kamineni, 2005).

\subsubsection{Methodological issues in lifestyle research}

Achieving clarity of understanding around the terminology related to lifestyle, psychographics, and the relationship of values, attitudes and AIO is not always straightforward. Lifestyle and psychographic terminology, and what is meant by each concept, can at times be unclear and one of the main issues other authors identify. Heath (1995) says that despite its introduction thirty years ago, psychographics is still one of the least understood and researched concepts in marketing.

The following paragraphs of this section therefore present a brief summary of psychographic measurement and the author's considered conclusions as to the appropriateness to this study.

\section{Conceptual clarity}

As one of the earliest contributors to psychographic research, Wells (1974) gives an extensive summary of the problems associated with lifestyle research. Wells brought together an early collection of readings on the subject, which highlighted a wide array of variation in interpretation. In its early days, the definitional problems were evident. Wells' review of the subject found thirty two definitions in twenty four articles, leading him to comment on the diversity and range of definitions, and sometimes conflicting opinions contributing authors seemed to hold on the topic.

In many ways, this lack of conceptual clarity still exists today. Bushman (1982), states that the term 'lifestyle analysis' leads to serious miscommunication. He warns that the term has no generally accepted definition and is operationally defined by researchers on an ad-hoc basis He also adds that the confusion comes from researchers implicitly subdividing and categorising lifestyles differently. Bushman's observations still appear to be relevant, evidenced in the literature review undertaken for this work.

Some authors appear to regard psychographics and lifestyle as an increasingly interchangeable term. Vyncke (2002) states that "the lifestyle concept has become so central, and the personality concept so marginal to psychographic research, that the latter is currently equated with lifestyle research" (p. 448). 
Psychographics is used by many authors interchangeably with AIOs (Tam \& Tai, 1998). Furthermore, many authors view lifestyle as a construct and psychographics as the operationalism of this construct (Wedel \& Kamakura, 2000).

\section{Robustness of construct and techniques used}

Criticism of lifestyle study generally focuses on opinions that the methods used are purely inductive and not guided by theory (Anderson \& Golden, 1984). A general conceptual problem exists in that central concepts are ill defined and have been criticised for vagueness (Anderson \& Golden, 1984) and whether they are sufficiently robust (Lastovicka, 1973).

Hoek, Gendall, and Esslemont (1996) present a summary of what they see as the inherent dangers of overall segmentation processes used in marketing and include psychographic techniques within the list of approaches. Their main criticisms are centred on flaws in base variables, analysis methods, defining the number and composition of segments, solution validity and problems of interpretation. However, they did not reject outright segmentation as a useful marketing approach. A main conclusion was that although the subjective component in segmentation cannot be avoided, confidence in whether segments identified are real will be increased with demonstrated sound techniques. These include conceptualisation based on adequate theory and data analysis techniques that are robust and appropriate.

\section{Values versus AIO measurement}

Although values studies may themselves be well grounded, the explanatory value to lifestyles and consumer behaviour is not high or well documented. Dichter (1986) discussed some of the 'pitfalls' he saw in using values and lifestyles to segment markets. He felt that "the complex and dynamic, sometimes contradictory nature of personality and attitudes prohibits any facile labeling" ( $p$. 312). It may also be wrong to assume values can be measured the same way across different cultures. For example, Rokeach's values study is regarded by some as reflective of middle class America and having a lack of empirical basis (Vyncke, 2002). 


\section{Stability of lifestyles}

Dichter (1986) points out how quickly lifestyle can change, (for example, following a major stressful life event), and also that one must assume lifestyle has a sociological, cultural, historical and political origin; for example, the lifestyle of women has changed over the years due in part to changes brought about by changed societal attitudes to a woman's role. He warned of values and lifestyle as being not easily observed and registerable classification systems but often misleading oversimplifications. Todd and Lawson (1998) also agree it is difficult to know to what extent groups are stable over time.

\section{Issues around tool development}

In regard to psychographic questionnaire development and item generation, some researchers have found difficulties in item generation and analysis, which includes problems such as lengthy questionnaires and because of the need for a wide range of respondents, questions that are difficult to construct and likely to be ambiguous (Hornik, 1990). Hornik also advises that lifestyle consumer profiles cannot be considered clear and mutually exclusive but rather, "distinct clusters of human qualities" (p. 38). Psychographic questionnaires are frequently expensive to design and collate (Heath, 1995).

Surveys are criticised for being too long, based on data required and scales based on scoring for every item of the questionnaire (González \& Bello, 2002). The length of the surveys results in flagging attention and opting-out. Moreover, as surveys are self administered, there is the added handicap of respondent lack of understanding, due to the often difficult questions (Gondalez \& Bello, 2002). Mitchell (1994) claims that the issues and problems of lifestyle measurement, reliability and validity, issues over scale development and interpretation, are all "magnified greatly if the more predictive bespoke psychological scales which focus on a specific product area are required" (p. 48).

In consideration of the above potential issues and problems, psychographic measurement technique still remains a useful and appropriate approach for this study. Firstly, it provides the most efficient way of measuring lifestyle holistically; secondly, it allows for a parsimonious and relatively seamless combination of health and marketing variables within a single study. The works of authors that highlight inherent dangers and potential traps for validity and robustness have served well in enabling sound and rigorous study design in this 
study. As a first step in that regard, the next section of this chapter discusses the approach to be used for this study.

\subsubsection{Choice of approach for the study}

The literature does not allow a clear picture as to whether a pure values approach, a mix of values and AIO, or an AIO inventory alone, provides the most useful information. In choosing an approach, the author's different viewpoints must be sorted through to reach a conclusion on the most viable and conceptually robust way to investigate pregnancy health behaviour lifestyle. It may be that in an AIO approach, attitudes are used as a proxy for values, or assume recognition of underlying values. Blackwell, Miniard, and Engel (2001), suggests 'lifestyle' is a more contemporary approach than personality and more comprehensive than values. Grunert, Brunso, and Bisp (1993) believe it is more useful to consider lifestyle as an intermediate level of a hierarchy of cognitive categories, with values being the most abstract. Blackwell et al. (2001) and Bruwer, Li, \& Reid, (2002) also advise caution, as values are relatively enduring, whereas lifestyles change more rapidly. Tilkidou and Delistavrou (2001) found that whilst the attitude-behaviour link remains an indisputable reality, personal values can provide a better understanding and lead to a better prediction of consumer behaviour.

Neal, Quester, and Hawkins (2002) provide useful advice. They state that AIO inventories were the initial attempts to measure lifestyle, but although they are an improvement from narrow demographic data, AIOs still proved too constricted. New psychographic or lifestyle studies include attitudes, values, activities and interest, demographics, media patterns and usage rates (within a specific product category). Neal et al. says that most studies use the first two or three dimensions, whereas the other dimensions are used to provide more complete descriptions of each group. Lifestyle measurement can also be undertaken with various degrees of specificity. Very general measures can be undertaken to investigate general lifestyle patterns, whereas other measurements can be activity or product specific. Using Wolburg and Pokrywczynski's (2001) classification of psychographic approaches as a guide, the aims of this study fit a general but context specific lifestyle approach. This means that respondents are classified into relatively homogenous groups to form a typology. Even though the focus of the study is health specific, some general AIO questions are relevant. For instance, Vyncke's (2002) suggested inclusion of media use is useful, given the study's objective of improving health communication. Rodgers, Chen, Duffy, and 
Fleming (2007) also advocate the inclusion of media variables, not typically used in health segmentation are they have been shown to be predictive of health behaviours such as 'diet and exercise'.

Psychographics is taken to be the operationalisation of the lifestyle construct, or the mechanism by which lifestyle is measured (Wedel \& Kamakura, 2000; Bruwer, Li \& Reid, 2002). This view takes into account the diversity and range of different psychographic approaches, whilst keeping lifestyle defined at the construct level. A combined approach that incorporates values, AIO statements, media and related dimensions, especially health, are used in this study, consistent with approaches used by other authors (e.g. Vyncke, 2002).

Because the focus of this study is context specific, several customised items and scales measuring the pregnancy context and health were also imported and incorporated. This narrowing did not widely restrict the inclusion of variables that would appear in more general psychographic studies, if appropriate. For instance, the value 'concern for physical appearance' would be very useful in a study of pregnant women as it may well relate to attitudes toward changing body shape and weight gain.

In conclusion, for the purposes of this study, lifestyle is the various modes of living and the patterns of consumption that go with them (Todd et al., 1998). The concept of lifestyle is useful as a way of examining the transitional health lifestyle of pregnancy, as it allows for a comprehensive approach and deep insights that the research objectives call for. Psychographics is an appropriate approach to measure the complex and multifaceted phenomena within lifestyle. Whilst several potential problems raised by other authors have been discussed above, this does not detract from the potential benefits of careful application of the method.

\subsubsection{MEASURING HEALTH AND HEALTH LIFESTYLE}

This next section describes the various approaches to measuring health. Health lifestyle is also defined, with pregnancy introduced as a health lifestyle.

\subsubsection{Health}

There is no universal agreement on the definition of health. Its meaning, in part, reflects the evolution of societal values and capabilities in terms of individuals, communities and the social environment over time. An individual's health also 
changes as one adjusts and adapts to a changing internal and external environment, including life stage changes. However, there is a broad understanding that health means a harmonious fusion of body, mind and spirit and it is best viewed as a multidimensional construct in its implications (Lowenberg \& Davis, 1994). This study focuses on factors surrounding health of pregnant women within a broad social context, including attitudes, values, transitional behaviour and circumstances surrounding the pregnancy itself.

Over the past fifty years, the World Health Organization's (WHO) definition of health, first formulated in 1946, and amended in 1948, has been the most widely adopted: "Health is a state of complete physical, mental and social well-being and not merely the absence of disease or infirmity" (WHO website). Contemporary criticism regarding the WHO definition now appears to centre around the lack of acknowledgment of social, economic and environmental influences on health. Two other declarations have also been significant. The Alma-Ata Conference on primary care in 1978 made several declarations regarding health: health is a basic right, governments have a responsibility for the health of their people, people should participate in planning and implementation of their healthcare and health is related to productivity. More recently, the Ottawa Charter defined health as "the ability to identify and realize aspirations, to satisfy needs, and to change or cope with the environment. Health is therefore a resource for everyday life, not the object of living. Health is a positive concept emphasizing social and personal resources as well as physical capabilities" (World Health Organisation, 1986).

Initiatives to measure health have been more successful than attempts to define it. With health a subjective state, it has different meanings from one person to another. Although a person may know what health or wellness feels like to them, it is often difficult to quantify, except by reductionalism. The popularity of more reductionalist approaches to health measurement is relatively recent and can be attributed to the medical and scientific models, made popular in the $19^{\text {th }}$ century.

Health lifestyle is the expression of collective attitudes and behaviours held and engaged in by an individual that determine the way they live in regard to their health. Health lifestyle can be examined within the general concept of consumer lifestyles, albeit with a specific emphasis on achievement and maintenance of 'health'. Lifestyle is an extremely useful construct in the health field (Walker et al., 1987), and there has been a significant amount of empirical work conducted 
supporting interrelationships; for example, between 'style of life' and health status.

In the context of health, lifestyle incorporates the behaviours over which an individual theoretically has control, including actions that affect a person's health risks (Ardell, 1977). Health values such as 'health consciousnesses', 'health locus of control' and 'health consumerism' underpin beliefs around health. These constructs provide utility in understanding and measuring an individual's likely involvement and interest in health affairs.

Another useful concept of how a lifestyle comes together is the concept of a 'causal recipe.' A causal recipe can be defined as the various combinations of causally relevant ingredients or conditions that come together to generate a particular outcome (Ragin, 2008). This suggests that consumers may 'mix and match' information, ideas, and health maintenance activities in the pursuit of a given outcome. Added to the mix, however, are the factors over which the consumer has less control, such as opportunities derived from their socioeconomic status.

The work of Bourdieu (1998) builds on the concepts of Weber's 'choice' and 'chance' and has been found to be useful by authors, such as Cockerham, Rutten, and Abel (1997) and Dumas and Laberge (2005), in defining health lifestyle. Cockerham et al. (1997) describe Bourdieu's 'habitus' as "knowledge of social structures and conditions that produce enduring orientations toward action that are more or less routine and when those orientations are acted upon, tend to reproduce structures from which they are derived" (p. 326). Bourdieu's 'habitus' recognises the concept of 'body' as a medium through which individuals act in accordance with their social position (Angus et al., 2007; Bourdieu, 1990; Casey 2001; Crossley, 2001). Likewise, Dumas and Laberge (2005) found the concept of 'habitus' a useful explanatory agent to "demonstrate the correspondence between the ways in which people treat their body and the culture of the social group to which they belong" (p.187).

Cockerham (2005) defines health lifestyle as "collective patterns of health-related behaviour based on choices and from options available to people according to their life chances" (p. 53). In agreement with Bourdieu's concept of 'habitus', Cockerham "assigns priority to chance (structure) over choice" (p. 54). A particular context of Cockerham's work is in defining the Russian health lifestyle 
and the problems generated by the social and cultural environment in Russia that restrict the achievement of health (Cockerham, 2000; 2007). The work is helpful to this study as it makes clear the link between daily lifestyle decisions and actions and subsequent health outcomes.

Several authors have undertaken to qualify the dimensions that make up a person's health, with authors converging on a set of health behaviours that describe an individual's health. Findings reveal that health is multi-dimensional, and corresponding scale developments use sub-scales to measure different dimensions. The scales derive from studies of specific health situations, with general agreement in most instances that they have applicability to the area of health in a specific context. The Health Promoting Lifestyle Profile developed by Walker et al. (1987), is a measurement instrument that spans what the authors propose as the main dimensions of health. The profile has been used successfully in many contexts by other authors studying certain aspects of women's health lifestyles (Sweeney \& Gulino, 1987; Wilson, Deane, \& Ciarrochi, 2005; Bond, Jones, Cason, Campbell, \& Hall, 2002; Hartweg \& Berbiglia, 1996; Duffy, Rossow and Hernandez, 1996). Therefore, as health is a central perspective in this study, with pregnancy the specific health context, several pre-existing measurement scales will be useful.

\subsubsection{Health psychographics}

Conceptual and empirical work to understand how consumers perceive and manage health has been undertaken by numerous researchers across a wide range of disciplines. In particular, these include medicine and allied disciplines (for example, dietetics, nursing, midwifery), sociology, psychology and gender studies. In the interests of brevity, this chapter only discusses studies considered particularly relevant to this study. It was discovered that, in a number of instances, there is theoretical overlap of interest areas that are common to each discipline only. However, in general, interest areas are discussed below within the context of their core discipline.

Many healthcare marketers still use demographics as the main source of consumer information, which is not necessarily a good predictor of similarity of behaviours. Furthermore, segmenting by behaviour alone makes assumptions that consumers are all alike in motivations and beliefs. Therefore, the use of psychographic measurement in health is aimed at understanding health behaviour patterns of a group of people from a broad and holistic perspective. The premise 
behind the use of psychographics in health is that if more is known about people's lifestyle, then the more effectively education, communication and health promotion can be employed to bring about healthier ways of life (Hornik, 1990). Ahmed and Jackson (1979) suggested that psychographics was relevant to the study of health and welfare policy decision making, and campaign construction in favour of demographics, as attitudes concerning welfare are independent of sociodemographic variables. Psychographics therefore is seen as adding additional explanatory power (Schmid, Rivers, Latimer, \& Salovey, 2008) to how consumers form into different groupings.

Health psychographics is the application of psychographic segmentation to the specific context of health and segments consumers along selected health dimensions (Hornik, 1990). This provides an opportunity for healthcare marketers to increase understanding of motivations, facilitators and barriers underlying health behaviour decisions and actions. As in other context specific psychographic studies that focus on certain variables, values and AIO relevant to a health situation are selected. The study may be general (that is a person's total health) or related to a particular health situation or challenge (for example, breast cancer screening, smoking cessation, exercise). Relevant to this study is the combination of factors that surround a particular life stage, and general health issues that are salient to that stage. Successful use of psychographics in commercial marketing has led to growing interest in psychographic research in health communication (Dutta-Bergman, 2004; Dutta \& Youn, 1999)

Hornik (1990) points out that when a psychographic study is devoted to a single behaviour (such as smoking), it is not necessary to depend on item diversity to get useful relationships. Hornik cites suggestions in Wells (1975) that investigators should concentrate on a limited set of relevant behaviour related dimensions. Hornik also suggests that psychographic elements be based on current relevant theories and findings. Anderson and Golden (1984) suggest that it is inefficient to develop psychographic models that ignore relevant behavioural theory. Furthermore, Hornik (1990) cautions that health behaviours (such as smoking consumption) should not be investigated on the psychographic variable alone, but in regard to other possible determinants, such as temporal orientation and demographic characteristics.

There are many factors and potential constructs to take into account when devising a health psychographic tool. Human behaviour is complex and the 
capture of all the influences that contribute to a health attitude or behaviour can be difficult to measure. A single measure may be included for differing reasons. For instance, alcohol intake is not only a health behaviour, but also a social one. This may lead to the inclusion of items measuring alcohol intake as a measure of a person's desire to be socially active. Hence the measure may be applied as a value or personality construct in a particular study, rather than as a measure of alcohol limitation to maintain health.

Furthermore, the meaning of 'lifestyle' in health can be conceived and operationalised differently, dependent on the discipline in which a study originates. The term 'lifestyle' in health is often applied loosely to imply the behaviours an author is interested in only. For example, Dejin-Karlson and Ostergren (2003) only selected lifestyle variables in a pregnancy study and only selected smoking and alcohol consumption in a study of pregnancy 'lifestyle'.

Segmentation approaches used in the elderly market share some commonality with the process by which the pregnancy market can be segmented. 'Gerontographics' (Moschis, 1996), defined as 'a life stage model' was developed to help marketers better understand the heterogeneous older consumer market. This work can be useful in understanding how a typology of pregnancy health lifestyle segments might be formed. First, the elderly market has historically been regarded as homogenous and with little consumption activity (except in health services). Secondly, as a person transits into the elderly classifications of The Household Lifecycle Model, lifestyle is affected not only by beliefs and expectations (and resources), but also health and physical capability. Similar to psychographics, Gerontographics uses psychological items as well as incorporating factors associated with biological, social and experiential aging. Moschis's Gerontographic segmentation (Moschis, 1996), was based on survey responses from 30,000 respondents over three years. Importantly, this work shows that a large set of lifestyle and health-related measures can be assessed in a systematic and insightful manner.

More generally, the academic literature is sparse on health psychographic studies. Hornik (1990) reports a study on time preference, psychographics and smoking behaviour, and Dutta-Bergman (2004) reports a study on healthy eating and social marketing using a psychographic approach. A broader search identified studies that were able to inform the development of this health psychographic/health lifestyle study. These include commercial applications of 
the methodology in health. The studies drawn upon to form this thesis, both academic and commercial studies, are summarised in Table 2.1.

Table 2.1. Health psychographics; empirical and conceptual studies.

\begin{tabular}{|c|c|}
\hline Study & Summary \\
\hline Bloch (1984) & $\begin{array}{l}\text { Examined prevention of health problems and maximization of } \\
\text { personal wellbeing through a lifestyles perspective. Concluded a } \\
\text { lack of work had been done to profile the wellness-oriented } \\
\text { consumer using psychographic measures. }\end{array}$ \\
\hline Hornik (1990) & $\begin{array}{l}\text { Study linked variables time preference, psychographics and } \\
\text { smoking behaviour. Findings demonstrated that psychographic } \\
\text { dimensions provided more meaningful and valid predictions of } \\
\text { smoking intensity. }\end{array}$ \\
\hline $\begin{array}{l}\text { Kraft and Goodell } \\
\text { (1993) }\end{array}$ & $\begin{array}{l}\text { Developed a wellness scale using psychographic measures to } \\
\text { segment the healthcare market. }\end{array}$ \\
\hline Moschis (1996) & $\begin{array}{l}\text { Studied the elderly and formulated Gerontographic segments. } \\
\text { Although not strictly a health psychographic study, his inclusion of } \\
\text { a health variable meant that health was incorporated as a major } \\
\text { influence into how segments were determined. Moschis's work has } \\
\text { been inspirational to the original idea of this study. }\end{array}$ \\
\hline $\begin{array}{l}\text { Trigg, Peterson, and } \\
\text { Meekers (1997) }\end{array}$ & $\begin{array}{l}\text { Studied key socioeconomic, demographic and psychographic } \\
\text { determinants in Zimbabwe to identify high risk sexual behaviour. } \\
\text { The study developed profiles of men and women practicing "risky } \\
\text { sexual behaviour" (as specified by various dimensions around } \\
\text { number of partners and condom use). Study found that excessive } \\
\text { alcohol intake, history of STD and embarrassment in acquiring } \\
\text { condoms were important factors. The authors believe that these } \\
\text { psychographic factors identified were more salient than any socio- } \\
\text { economic or demographic factors and would be useful for targeting } \\
\text { condom use in similar populations. }\end{array}$ \\
\hline $\begin{array}{l}\text { Dolinsky and } \\
\text { Stinerock (1998) }\end{array}$ & $\begin{array}{l}\text { Conducted a study across three cultural groups in the United States } \\
\text { (African-American, Hispanic-American and Anglo-American). } \\
\text { Identified distinct differences in the importance attached to various } \\
\text { healthcare attributes across the three groups. }\end{array}$ \\
\hline $\begin{array}{l}\text { Granzin, Olsen, and } \\
\text { Painter (1998) }\end{array}$ & $\begin{array}{l}\text { Identified segments in relation to health-related offerings. } \\
\text { Referencing Pender's model they suggested that measurement } \\
\text { could not just use health promotion behaviours, but also should } \\
\text { examine the range of health repressive behaviours and } \\
\text { participation and avoidance of these behaviours. }\end{array}$ \\
\hline Potomac (1998) & Profiled the 'wellness segment'. Suggested \\
\hline
\end{tabular}




\begin{tabular}{|c|c|}
\hline & $\begin{array}{l}\text { psychographics study ties behaviours to attitudes, beliefs and } \\
\text { aspirations in a valuable way, psychographic segmentation has a } \\
\text { major drawback for healthcare marketers in that studies are based } \\
\text { on consumer purchasing behaviours not on health behaviours. }\end{array}$ \\
\hline $\begin{array}{l}\text { Porter Novelli; } \\
\text { 'Healthstyles' } \\
\text { (Maibach, Maxfield, } \\
\text { Ladin \& Slater, } \\
\text { 1996). }\end{array}$ & $\begin{array}{l}\text { Commercially generated, "Healthstyles" form part of an annual } \\
\text { survey conducted by Porter Novelli each year. Porter Novelli claims } \\
\text { that the HealthStyles database was developed because "health } \\
\text { decision-making does not follow the same pathway as consumer } \\
\text { purchase behaviours" (Anon b 1998). The 'Styles' collection of } \\
\text { surveys is comprised of 'HealthStyles', 'ConsumerStyles' and } \\
\text { 'YouthStyles'. HealthStyles examines the American public's health } \\
\text { orientation and practices. The HealthStyles database is used within } \\
\text { commercial contexts defining and targeting different health } \\
\text { audiences and is proprietary. }\end{array}$ \\
\hline Hagoe & $\begin{array}{l}\text { Clustered health lifestyles in regard to similar behaviour patterns of } \\
\text { Israeli women. They found three groups, a 'health-enhancing' } \\
\text { cluster, an 'inactive' cluster and an 'ambivalent' cluster. The } \\
\text { authors found the pattern of a three cluster finding replicated the } \\
\text { work of Abel (1991). Of interest was the finding that the group } \\
\text { that revealed the most health promoting behaviours had the most } \\
\text { privileged circumstances (education, work status), those with the } \\
\text { inactive or health compromising patterns had the poorest socio- } \\
\text { cultural circumstances; the ambivalent group (made up of some } \\
\text { heath-comprising behaviours but mixed with health enhancing } \\
\text { behaviours) were incircumstances between the two previous. Their } \\
\text { conclusion was that the data "strongly supported the Weberian } \\
\text { notion that life chances are related, if not determinate of life } \\
\text { conduct, that is, behaviours" (p. 179) }\end{array}$ \\
\hline $\begin{array}{l}\text { Dutta-Bergman } \\
\text { (2003) }\end{array}$ & $\begin{array}{l}\text { Examined the profile of healthy versus non-healthy eating along } \\
\text { demographic, psychographic and communicative variables. Study } \\
\text { found healthy eaters were environmentally conscious and health- } \\
\text { oriented, suggesting an underlying theme of personal and social } \\
\text { responsibility. Healthy eaters also had a greater information } \\
\text { orientation while unhealthy eaters were more entertainment } \\
\text { oriented. }\end{array}$ \\
\hline $\begin{array}{l}\text { SPARC; 'Obstacles to } \\
\text { Action' Physical } \\
\text { Activity and } \\
\text { Nutrition study } \\
\text { (2003) }\end{array}$ & $\begin{array}{l}\text { A recent New Zealand study by Sport and Recreation New Zealand } \\
\text { (SPARC 2003) and the Cancer Society of New Zealand used the } \\
\text { Porter Novelli HealthStyles methodology as a basis for segmenting } \\
\text { physical activity and nutrition behaviours of New Zealanders. The } \\
\text { Physical Activity and Nutrition study investigated health } \\
\text { behaviours, motivations, attitudes, intentions and barriers and } \\
\text { defined six clusters that have informed a social marketing }\end{array}$ \\
\hline
\end{tabular}




\begin{tabular}{|l|l|}
\hline & $\begin{array}{l}\text { campaign 'Push Play'. The Push Play campaign aimed to target } \\
\text { selected segments with specific health communication messages to } \\
\text { increase physical activity. }\end{array}$ \\
\hline Kolodinsky and & $\begin{array}{l}\text { Segmented 'overweight Americans' and found five clusters: highest } \\
\text { Reynolds (2009) }\end{array}$ \\
$\begin{array}{l}\text { Getting Best Results (13\%); and Doing OK (12\%). They suggested } \\
\text { that through the identification of these five segments, social } \\
\text { marketing campaigns can be better targeted. }\end{array}$ \\
\hline
\end{tabular}

In summary, the studies described above provide a useful background as to how values, attitudes and 'styles of living' impact on how health lifestyles are played out. The authors above have demonstrated that in respondents within the context investigated, specific segments with distinct homogeneous attributes could be identified. Health psychographics therefore can be considered a very viable methodology for this study's aims and objectives. The next sub-section discusses health lifestyle and psychographics within the context of pregnancy.

\subsubsection{PREGNANCY HEALTH AS A LIFESTYLE}

Lifestyle factors are already known to be an important variable in the quality of the childbearing experience and health outcomes for mother and infant (Chomitz, Cheung \& Lieberman, 1995; Clissold, Hopkins \& Seddon, 1991). Lifestyle choices during pregnancy have long been of interest to clinical researchers and those charged with improvements in the health outcomes of populations. Quality outcomes are desired on several levels: individual, health service and societal. For example, an individual desired outcome for a woman may be a positive birth experience that achieves goals for labour within her birth plan; whilst societal, may be a reduction in maternal and infant morbidity statistics. Some woman may even view having a baby of a preferred sex, as a 'quality' outcome. Furthermore, the childbearing period is often the first time young healthy women interact with a healthcare system for a continuous and sustained period of time. Their interest and receptivity is often heightened, due to the novel changes they themselves are experiencing and concern for themselves and their child (Gennaro \& Fehrer, 2000).

\subsubsection{Consumer decision making and pregnancy health}

In order to adapt to the transitional period that pregnancy encompasses, pregnant women are called to make decisions about their health lifestyles. Therefore, consumer decision making models are directly relevant to understanding the choices that may be made for pregnancy health lifestyle 
expression. Health psychographic segmentation is a directly applicable and helpful methodology for further refining the various modes of consumer decision making in pregnancy. The outcome of such approaches can then be used to guide strategies and communication initiatives aimed at improving health outcomes.

Consumer decision making models are derived from four main perspectives: economic, passive, cognitive and emotional. The cognitive model views consumers as problem solvers, in an information-processing system (Schiffman, Bednall, O'Cass, Paladino, \& Kanuk, 2005, p.445). However, for many decision making situations, emotional factors are influential (Schiffman, et al, 2005). In the case of pregnancy, which is a deeply personal transitional lifestyle, both cognitive and emotional factors are particularly applicable.

The Consumer Decision Making Process model (CDMP) (Schiffman et al., 2005, p.445) is used extensively in marketing to describe extended (and routine) purchase decisions. The model mainly favours consumers as making rational decisions in complex situations (although a little emotion can be accommodated). Inputs include the socio-cultural environment and marketing activities. Need recognition generates problem solving activity, a search for information, evaluation of alternatives and finally, a purchase decision. The higher the level of situational or enduring involvement or novelty, the more likely the process will be complex. Within the context of this study, it is assumed that for the most part, complex consumer decision making occurs that is rational and embedded in high situational involvement. As a high involvement and novel situation, the transition to first time motherhood implies that a significant degree of information acquisition would be needed in order to negotiate the passage through (Gennaro \& Fehrer, 2000; Hirschman, 1991; McKenzie, 2001).

In fact, Hirschman (1991) argues that a new baby is a major 'discontinuous innovation' with far reaching effects on consumption. The potential types and sources of information sought, media preferences for information, reflection undertaken and perceived responses are considered and evaluated. If the notion of a transitional health lifestyle as a purchase or product adoption is agreed on, the CDMP model can be applied to the pregnancy situation, where a range of novel or first time behaviours are selected over other possible alternatives. In this context, health information, either distributed by health professionals and/or actively sought by women, are aids to decision making and empower consumers 
to make choices. Post purchase evaluation could feasibly be the assessment and reflection engaged in when determining the effect of the changes made, on usual activities of living and lifestyle patterns.

Consumer decision making in health is embedded in a wider context than is typically the case for most other decision making situations. Selection of lifestyle behaviours is an integral part of responses to the socio-cultural environment and existing patterns of a person, as well as the communication fields that interact with the person (Cockerham, 2005). This, therefore, implies that not all consumers may be equally empowered or equipped with capabilities and resources to make efficient decisions. Arguably, however, Cockerham's (2005) notion of structure (chance) over choice in health lifestyle is potentially tempered by the provision of pregnancy related information, increasingly available ethnically targeted services and free public maternity services that provide oneon-one-contact with a health provider in New Zealand. This might, to some degree at least, increase informed decision making opportunity and smooth access to health support and information, in order to make health promoting changes that are usually advantaged by a higher socio-economic status.

\subsubsection{Pregnancy health psychographics}

There have been few segmentation studies of the pregnancy market from a conventional marketing segmentation approach. Miaoulis (1989) conducted a segmentation study on the basis of benefits sought from preadolescent pregnancy, and identified five benefit segments. The author states that by identifying the main underlying motivations for becoming pregnant within each consumer cluster, a foundation can be laid for preventative health marketing strategies. There are no studies evident in the literature of pregnant women that apply a health psychographic orientation by using an extensive application of variables in a general nulliparous pregnant population.

Contemporary obstetric practice categorises or segments 'patients' according to existing clinical classification systems, comprised of variables such as demographics, obstetric status, and pathology or pre-existing health status (see for instance, Roodenburg, Wladimiroff, \& Prechtl, 1991). The continued application of this type of categorisation remains crucial to clinical diagnosis and management. Medical studies however, whilst addressing lifestyle factors as causative agents for morbidity, cannot contribute a comprehensive or holistic evaluation that achieves the goals of healthcare marketing, as a medical 
paradigm focuses primarily on medical or health interventions only. The social sciences, particularly psychology, sociology (family and relationship studies) and gender studies (feminism and the changing role of women and motherhood in society), have contributed much to the background context of the study, and have helped form the foundation premises for the study.

Evidence suggests that studies investigating health behaviour in high risk pregnancy, or factors that impact to make pregnancy high risk, have been studied more than low risk, or normal pregnancy, despite low risk pregnancies been the norm (Smith, 1999). Many of the low risk studies reported are qualitative, using approaches such as interpretative phenomenology to investigate meanings of pregnancy in small respondent samples (Smith, 1999). Indeed a lack of frameworks or theories specific to low risk pregnancy limits the applicability of previous work to the underlying framework for the thesis. This study assumes that low risk pregnancy is a normal developmental life event and not a disease or illness. Therefore, in order to avoid the potential limitations of examining health lifestyle through a medicalised paradigm, a wellness or holistic approach to health has been preferred, with some respondents recognised as falling into the higher risk end of the spectrum. Provision has been made for this by accommodating high risk measures. However, high risk women may share many of the choices for health lifestyle that their low risk counterparts experience. This applies particularly if their condition requires surveillance and monitoring only, rather than direct medical intervention.

The premise that maternity consumer heterogeneity exists, with corresponding variations in transitional lifestyles amongst maternity consumers, is critical to this work. The existence of discrete lifestyle groups is, in all likelihood, not new to the maternity market, as is known intuitively by many practitioners. During the course of practice, practitioners themselves would have experienced many different childbearing consumer contexts and come to recognize patient groupings. One early example would be the 'home birth' group. The philosophies and activities of this group of women heralded resurgence in lobbying and consumer demand for home birth during the 1970's and early 1980's, at a time when medicalised maternity care deemed home confinement unsafe (Leap \& Barclay, 2001; Stojanovic, 2008). It can be argued that this group of women exhibited a unique set of attitudes, opinions and behaviour characteristics. These formed the basis of a discreet lifestyle group, identifiable by their demand for non-medicalised care and hospital confinement. This created a demand for and 
consumption of goods and services that for the time were considered very much niche. Consumer demand from within this market led to the formation of practitioner offerings with appropriate positioning and targeted products (Leap \& Barclay, 2001).

Additionally, within current practice, some midwives actively target specific consumer groups or promote service approaches that cater to specific needs and desires. This meets an evolving and increasingly diverse market that exhibits distinct expectations and preferences for the management of their maternity experience. Māori and Pacific midwifery practices, for example, aim to facilitate an experience that recognises the cultural childbearing traditions of these groups. As a further example, media attention has been given to a group of women referred to as 'too posh to push', named after Victoria Beckham as Posh Spice. Women in this grouping are apparently reluctant to labour, especially through the second stage. By opting for a caesarean section, they are said to believe that they will avoid unnecessary damage to their anatomy. If this group is in fact an identifiable lifestyle cluster, it leads to many interesting questions. If these consumers are ambivalent about birth without intervention, or in fact reject it, their goals are in conflict and compete with what practitioners state they are committed to. If a change in these consumers' attitudes is believed advantageous, services that target this group would need to consider selling the concept of normal birth through a social marketing framework.

Overall, this study sets out to effectively blend pregnancy lifestyle as it has been studied and interpreted in health, with the meaning of lifestyle as it is conceptually defined in marketing. Although the two disciplinary orientations have not been merged from a theoretical standpoint before, in practice settings, it has not been uncommon. For instance, health practitioners have long given advice to pregnant women on non-health consumption decisions (baby clothes and equipment), whilst marketers increasingly promote to consumers products that were once specialised health products (apnoea monitors) and disseminate health information through popular press magazines.

Therefore, the study of the transitional health lifestyle of pregnancy is offered much by a health psychographic approach, as it enables multiple variables to be accommodated and synthesised in a comprehensive manner. It has the ability to accommodate and address a comprehensive range of variables from both marketing and health disciplines. 


\subsubsection{SECTION SUMMARY}

This section has discussed the concept of lifestyle in marketing. The measurement of lifestyle is a key tool marketers use, as lifestyle is expressed through information use and consumption behaviours. Therefore, it has utility as a conceptual framework for this study and as a way of determining different groups of consumers, according to their health lifestyles in pregnancy. Consumer decision making is useful in explaining how lifestyle related consumption decisions are made through rational reasoning and/or emotional and hedonistic inputs. However, because pregnancy for first time motherhood is a novel situation, characterised by high information need, rational and complex decision making has been determined as the dominant influence in the process.

Psychographics is an effective tool for lifestyle measurement. It provides beneficial insights and tends to be inherently holistic in its approach. The methodology is seen to be particularly applicable to pregnancy as a high involvement transitional lifestyle phase of life and equipped to capture the diverse consumer groups that make up this market.

This section also described the various approaches to measuring health, a complex and multi-faceted phenomenon. Health lifestyle was also defined, along with a focus on how health lifestyles can be measured. Holistic measurement comprises of assessing the multiple underlying influences along with manifest behaviours. Cockerham (2005) noted that health lifestyle is not merely an expression of consumer choice, but also heavily affected by life's circumstance. Therefore, several socio-cultural factors will impact on how decisions are made. It was proposed that pregnancy could be seen as a distinct health lifestyle. The components of a pregnancy health lifestyle are discussed in the next section.

\subsection{COMPONENTS OF A PREGNANCY HEALTH LIFESTYLE}

\subsubsection{INTRODUCTION}

Before a psychographic segmentation can be undertaken, it is essential to identify the underlying factors that make up the type of lifestyle in focus, so they can be measured and apportioned appropriately into their discreet dimensions (Moschis, 1996; Wells, 1974). This section discusses the components of the transitional health lifestyle of pregnancy that appear in the multi-disciplinary literature. The literature review reflects on and discusses conceptual and empirical work from 
multiple disciplines, as the study sought to focus on and capture salient variables that together make up the pregnancy health lifestyle. In order to be as parsimonious as possible, the works were categorised into three main groupings. Group one introduces factors that are present before and at the onset of pregnancy, that is, pre-existing dimensions (made up of demographic, preexisting values and traits and those pertaining to the pregnancy event or circumstance). Group two explores factors that influence and drive transition through this life stage (information, main or overarching attitudes toward pregnancy health and potential motivators and barriers or constraints). Group three divides manifest behaviours of pregnancy health lifestyle into two subsections: physical and psycho-social. For clarity, attitudes that relate toward a specific health behaviour are discussed with the behaviour itself, rather than in the broader 'pregnancy attitudes' section. Finally, an understanding of what would make up a positive pregnancy transition, in regard to health and how this could be measured (in the absence of more conventional birth outcome indices), was sought and is discussed.

The author acknowledges that in some areas (for instance, nutrition, smoking, adolescent pregnancy), there are extensive bodies of work beyond the scope of this study to summarise comprehensively. The discussion focuses specifically on sources considered to be relevant to the aims of the study, in particular the measurement of the pregnancy health lifestyle from an integrated health and marketing perspective. The accumulation and interaction of antecedents, information use, attitudes and subsequent health behaviours leads to the transitional pregnancy health lifestyle.

\subsubsection{PRE-EXISTING DIMENSIONS}

\subsubsection{Demographics}

Several demographic factors are known to influence pregnancy health (Chin, Monroe \& Fiscella, 2000; Joseph, Liston, Dodds, Dahlgren, \& Allen, 2007). Demographic attributes impact upon lifestyle choices, as they can define and shape the resources through which such choices are made. Age, ethnicity, family structure and socio-economic position (including income, education and work) all have relevance to pregnancy health measurement (Chin et al., 2000).

There is an age range believed to be the best for pregnancy and childbirth, both physically and socially (Gustafsson, 2001). Whilst this is controlled and dictated 
by biology to a large extent, changes have occurred over the past decades, particularly at the age extremes. The average maternal age of first child has steadily risen in the Western world over the past two decades (Ministry of Health, 2004), with the average age of first maternal age at birth in New Zealand increasing. An increased age at first baby may be associated with lifestyle choice, availability of effective contraception and abortion, importance of establishing a career in early working life and other economic factors. Technology also allows for fertility treatment in older women who would not normally be able to conceive. Older motherhood can be a risk due to the possibility of biological disadvantages complicating or causing stress in a pregnancy (Stein \& Susser, 2000). In regard to social impacts, Friese, Becker, and Nachtigall (2008) discuss technology producing a unique group, who facing motherhood for the first time at the end of their reproductive cycle, are forming a 'new middle age'. The redefinition of Full Nest I by Schnaninger and Lee (2002), into normal and late onset of Full Nest 1, acknowledges that consumption differences exist between the two groups. Adolescent pregnancy is often associated with negative health factors, such as increased social risk (Cagampang, Barth, Korpi, \& Kirpi, 1997; Miaoulis, 1989; Dobrzykowski \& Noerager Stern, 2003; Myers, Heazell, Jones \& Baker, 2006; Amy \& Loeber, 2007) and substance abuse (Berry, Shillington, Peak \& Hohman, 2000).

Ethnicity can be linked to health outcomes due to association with particular socio-economic position and disparities. In New Zealand, Māori have less optimal pregnancy and birth outcomes than 'white' infants, as assessed by birth weight (Ministry of Health, 2004). This is similar to the United States where infants of African Americans do not fare as well as 'white' infants (Geronimus, 1996). Although persisting disparities in outcome across ethnicities is essentially unexplained, low socio-economic groups and the 'cycle of poverty' are evident.

Diversity of household and family structure, in which a pregnant woman lives, is evidenced by the complexity of modernised Household Lifecycle Models (Gilly \& Ennis, 1982; Murphy \& Staples, 1979). Living situation is an influential variable in health lifestyle as a potential enabler or barrier to engaging in pregnancy health promotion behaviours, and as a source of support or stress.

Household socio-economic level is a significant influential variable in lifestyle, as it can dictate a person's access to information, education and resources (for essential and discretionary expenditure), as well as being influential in 
educational attainment and employment opportunities. Low socio-economic status has been repeatedly linked to poor outcomes in several health contexts, including maternal/fetal morbidity and prosperity (Joseph, et al., 2007). Chin et al., (2000) claim that if lifestyle and behavioural risk factors were not influenced by societal factors, they would be evenly distributed throughout the population. They note further that little attention has been given to why lifestyle behaviours cluster according to social class. Social circumstance can influence health lifestyle through multiple pathways: limited opportunities for self-fulfillment, financial constraints, health beliefs, self-efficacy and social support.

Because of social changes and economic demands on households most women remain in employment well into their first pregnancy (Lindsay, 2004). Decisions regarding reduction or cessation of work can be complex, particularly as these are made at a time of life stage change, associated with major expenditure (entry into Full Nest 1). The type of work, a woman is employed in, may affect employment decisions; such as, a potentially dangerous or demanding physical environment, high degree of physical energy required, inability to reduce hours of work without resigning, or a partner's unstable employment situation. Work environments also expose the woman to another socialisation group and potential support group beyond family and friends. Lindsay (2004) examined pregnancy and 'spaces of everyday work'. $85 \%$ of women now work in pregnancy and, although the benefits of pregnancy and work are mainly positive for the infant, there are large discrepancies on the effect of work for women.

A person's wider environment and community can also have significant influence over health status and behaviour and therefore act as influencing variables. Geography and domicile have proven to be useful considerations in general psychographic studies (Gunter \& Furnham, 1992). Access to professional resources may vary across urban and rural regions, thus influencing choices and decision making. Like consumers tend to congregate within regions and therefore access and consume similar products and services.

In summary, age, household structure and socio-economic status, ethnicity, work and domicile all impact in one way or another on pregnancy lifestyles. A robust conceptualisation needs to take these factors into account when segmenting and profiling women in this transitional lifestyle. 


\subsubsection{Pre-existing values and traits}

This sub-section discusses additional values and personality traits that have been shown to impact on health lifestyle and the motivations behind actions. There were no studies found that have applied these values specifically to a pregnancy context, and the studies reported are all based upon non-pregnant populations.

\section{Values and health}

There are several values identified that can indicate the meaning of health to a person. 'Health as a value' reflects the importance placed on health; whereas, 'Health Consciousness' is a measure of intrinsic and enduring involvement (Park \& Mittal, 1985; MacInnis, Moorman, \& Jaworski, 1991; Dutta-Bergman, 2003). 'Health Consumerism' reflects a consumer oriented philosophy and commitment to actively participate in consumption choices (Dutta-Bergman, 2003). It also drives a person's behaviour and the extent to which they will control both their relationship with healthcare providers and their care. Health Consumerism is made up of three behaviours: reading, questioning and complaining (DuttaBergman, 2003; Root \& Browner, 2004). A person who has a high level of 'Health Consumerism' would be expected to have high involvement, participate in the provider client relationship and willing to critique the quality of their care.

'Health Locus of Control' (HLOC) has proven to be a useful framework to measure the extent to which people see themselves responsible for their own health; or whether they perceive forces external to them are responsible for control over their health (Wallston, Wallston \& DeVellis ,1978). Health Locus of Control can be divided into Internal and External factors (Powerful Others and Fate) that would a-priori influence health and are thought to remain relatively stable through life. A woman's Health Locus of Control may well be dependent on whether she perceives pregnancy as a natural event over which an individual has a high level of control and responsibility (thereby high Internal), a state where health provider oversight and intervention is essential (high External: Powerful Others), or dependent on fate (high External; Fate). A woman's HLOC may also be seen as an antecedent to information seeking, with self-efficacy an influencing variable in a woman's confidence in her ability to manage her own pregnancy health.

Extension of the HLOC concept has been undertaken by Labs and Wurtele (1986) to develop a 'Pregnancy Health Locus of Control' (PHLOC) and a 'Fetal Health Locus of Control' (FHLOC). Haslam, Lawrence, and Haefeli (2003) found that a high Internal FHLOC was associated with more positive health behaviours during 
pregnancy, concerning intention to breast feed, taking iron and vitamin supplements and not smoking. Kruse, Zweig, and LeFevre (1988) investigated health behaviours during pregnancy, and found that a Powerful Others HLOC was associated with a variety of social and demographic characteristics and with several health related behaviours during pregnancy. The HLOC construct appeared central to this study's objectives and was incorporated into the underlying conceptualisation.

Higher level values operate as guiding principles to achieve desired goals or end states and are held to be relatively enduring. High level values are thought to remain stable through life changes and events; therefore, would remain stable through pregnancy. Kahle's List of Values (LOV) (1983) was found to be the most useful scale for the measurement of core values, in a health situation, as it is related to family and social interactions and based on Maslow's Hierarchy of Needs (Divine \& Lepisto, 2005). Divine and Lepisto (2005) utilised Kahle's LOV in a study of the 'healthy lifestyle consumer'. They compared the more hedonistic values of fun, enjoyment and excitement, with regular exercise and healthy diet, as dimensions of a health promoting lifestyle; they found excitement to be significantly negatively linked.

'Time orientation' is a personality trait that relates to a consumer's marginal rate of substitution between consumption in any two time periods: now and the future, with a positive preference favoured if a gain is preferred earlier rather than later (Hornik, 1990). A time preference model involves concepts of risk and uncertainly, as the future is unpredictable. Ability to plan ahead, including time on hand and less role overload and stress, has also been found to be a key factor in the pursuit and maintenance of a healthy lifestyle (Hornik, 1990; Divine \& Lepisto, 2005)

Dutta-Bergman (2003) suggested that 'environmentalism' is a significant factor in the health conscious consumer with an enduring involvement, as someone high in the latter would be interested in the impact of environmental events on health. An environmentally conscious attitude would also favour environmentally friendly products and practice pro-environmental behaviour. Tilikidou and Delistavrou (2001) found those high in materialism (the importance of and the level to which people pursue the things they want) were lower in inclination to recycle (an 'environmentally friendly' activity). Materialism value and possession orientation may also be significant due to the potential economic impact of a baby on 
disposal income and future potential wealth accrual. Delayed Full Nesters (Schnaninger \& Lee, 2002) may be more possession oriented, having enjoyed a longer phase of wealth accrual.

The drive to reduce the gap, between current self-image and ideal or desired selfimage, is a forceful motivator in the pursuit of products and services which are perceived as closing the gap. For women with a strong orientation to weight control, a desire to align with a societal view on the ideal sexual female shape, or a high concern for physical appearance, a situation that takes control away (particularly those directly due to a gravid uterus) could be regarded as threatening.

The strength of a person's belief, in regard to certain values and concepts, has been found to be influential over health as they can be strong reinforcers of behaviours and guiding influences. 'Religiosity', the extent to which a person believes religion influences their life, appears to be positively associated with health promoting behaviour (Schieman, Nguyen, \& Elliot, 2003). Religiosity can also be an important influence on beliefs and practices in childbirth (circumcision, abortion, childbearing outside of marriage) and one's world view (Ellison \& Levin, 1998; Ellison, Boardman, Williams, \& Jackson, 2001).

In a related sense, superstitious beliefs can strongly influence consumer behaviour (Rudski, 2003). Those high in superstition are likely to be higher on Fate or Chance Locus of Control (Mowen \& Carlson, 2003). Liamputtong et al., (2005) emphasised the relationship between culture and the importance of adhering to superstitious practises, often expressed as rituals. Examples of superstitious practices in pregnancy are avoiding foods that cause problems in labour (for example, eating a whole banana will cause the baby to become obstructed; Liamputtong et al., 2005, p.143), or the couvade in Slavic societies (Oinas, 1993).

\section{Personality traits and dispositions and health}

'Mastery' and 'Self-efficacy' are considered conceptually very similar (Ellison et al., 2001; Schieman et al., 2003). They demonstrate the perceptions one has of their ability to perform a certain action that will result in facilitating a certain goal (Pearlin and Schooler, 1978; Turner and Llloyd, 1999; Ellison et al., 2001; Schieman et al., 2003; Pearlin, Nguyen, Schieman and Milkie, 2007). The concepts are used extensively in health because of their "robust capacity to 
protect health and well-being" (Pearlin et al., 2007, p. 165). Self-efficacy in pregnancy is particularly important, as it is related to a woman's confidence in her ability to achieve goals and make effective health decisions.

'Trait anxiety' is a person's predisposition to anxiety, which can affect health lifestyle by increasing one's tendency to worry (Spielberger, 1983). A person with a high trait anxiety could be more at risk for anxiety-related (state anxiety) and depressive illness during pregnancy. 'Approach-avoidance' (Elliot, 2006) is defined as the extent to which a person will approach or avoid a potentially stressful or fearful situation. Those with a high approach-avoidance would therefore not willingly seek or wish to gain information or experience that carries a threat of danger. Approach-avoidance may moderate a woman's need to know about her particular status, in contrast to a desire to avoid a potentially stressful or fearful situation by avoidance of information. Some women may also not wish to have information that they can do little about; they would rather wait until birth to know that something is wrong with the baby, if little can be done during the pregnancy. A 'dislike of change' could also affect how readily one accepts the changes pregnancy heralds.

'Achievement motivation' is defined as a trait type disposition that affects how one engages in, appraises, and performs at an achievement situation (Sideridis, 2007; Elliot, 1997). Although achievement motivation is usually associated with academic or work pursuits, the relevance of goal setting in pregnancy and need to achieve desired outcomes for birth, could feasibly be mediated by it Failure to achieve goals may result in disappointment if the pregnancy and birth course deviate, and this has been linked to post-partum stress (Salmela-Aro, 2009).

\section{Cultural practices, family and female role orientation}

Childbirth has many embedded cultural norms and practices; a woman's affiliation and commitment to a particular culture can be an important influence on values, beliefs, attitudes and practices (Ayaz \& Efe, 2008; Liamputtong et al., 2005). Many practices observed or learnt vicariously in pre-childbearing years can provide strong cues for roles and behaviour, as well as regulating lifestyle and health practices. Similarly, interaction with one's culture means that a woman is exposed to a social or support network with specific viewpoints and practices. This may produce conflict or tensions if a woman attempts to practice traditional methods in a health system where her culture is not the dominant one (Liamputtong et al., 2005). 
With regard to acculturation, McGlade, Saha, and Dahlstorm (2004) discuss a paradox, whereby Latino (Mexican) immigrants to the United States should be an at risk group for pregnancy outcomes, based on their low socio-economic rating. However, the statistics shows despite disadvantage, their outcomes are the same as for non-Latino women. This is thought to be due to the informal prenatal care delivery system consisting of friends, community members and lay health they access. They are also influenced by 'The Healthy Migrant Theory' (McGlade, Saha, \& Dahlstorm, 2004). McGlade et al. suggest that the Mexican culture has protective factors, such as healthy traditional dietary practices, and a norm of 'selfless devotion' to maternity that enables new migrants to resist the temptation to adopt negative behaviours in a host country, such as smoking and drug abuse. As the level of acculturation increases, this system will not be as significant and disparity will be shown. Bond et al. (2002) reported similar findings in a study of Hispanic women, where women considered bicultural exhibited lower scores on health promoting behaviours than women with lower levels of acculturation.

New Zealand is a multi-cultural society (Ward \& Masgoret, 2008). and the ability to express one's cultural preferences in childbearing is gaining increasing focus with the emergence of culturally positioned service offering. For example, the emergence of Māori midwifery services whose goals are to facilitate and empower Māori women to practice traditional Māori childbirth practices (Papps \& Ramsden, 1996). Sensitivity to bi-culturalism and the delivery of health services that accommodate different cultural needs, as a means to improved health outcomes, is a well documented objective of the healthcare sector and current Government policy, through The Treaty of Waitangi (Durie, 1989).

A woman's more intimate environment, the family and important others, can play key roles as influencing agents in health attitudes and practices. Social support has been shown to have a protective effect on health (Almeida, Molnar, Kawachi, \& Subramanian, 2009) and is often generated by a person's family group. Changing household structures can influence the makeup of familles and the dynamics associated with interactions, felt commitment and expectations (Yoo, 2006). The concept of 'familism' is related to the strength of family ties (Villarreal, Blozis, \& Wildaman, 2005; Luna et al., 1996; Steidel \& Contreras, 2003), with a person higher in familism more likely to act in accordance with what would please the family. 
A woman's perception of a female's role in society can have an effect on values, family orientation and lifestyle (Tam \& Tai, 1997; Sin \& Yau, 2004). Female role orientation can also influence attitudes and beliefs related to obligation to produce and nurture children, right to independence and achievement, and work combined with motherhood (Tam \& Tai, 1997; Sin \& Yau, 2004). Over the past decades many 'taken for granted' expectations and roles have been challenged, particularly since the growth of the Women's Rights Movement of the 1960's (Engelhardt, \& Prskawetz, 2004; Mason \& Lu, 1988).

In summary, this sub-section has reviewed the antecedent variables that exert influence over a woman at the time of pregnancy. As many of these variables are value based or personality traits, they are enduring and can exert a strong influence over present and future behaviour.

\subsubsection{The circumstances around the pregnancy}

\section{Pregnancy intendedness or wantedness}

A major issue in the literature is how to define pregnancy intention, with many studies undertaken to measure the multi-dimensionality of pregnancy intendedness (Speizer, Santelli, Afable-Munsuz, \& Kendall, 2004; D'Angelo, Gilbert, Rochat, Santelli, \& Herold, 2004). Authors believe that it is a complex and often inconsistent construct that reflects ambivalence toward transition into motherhood for many. Conventional measures of pregnancy intendedness measure pregnancy by unwanted (unintended) or intended, or having a pregnancy wish of some description; such as, 'around the right time' or mistimed (Speizer, Santelli, Afable-Munsuz, \& Kendall, 2004). D'Angelo et al. (2004) discuss that most studies on intended versus unintended pregnancy, combine mistimed versus unwanted together as the category unintended. Based on their findings, they cautioned against this categorisation, as it masks apparent differences. Pulley, Klerman, Tang, and Baker (2002) found similar results, leading them to conclude that mistiming is not a 'unitary' construct; they recommended that any future research on pregnancy intention examine the extent of mistiming and consider alternatives to traditional definitions of intendedness. They also suggested that the three broad classifications of pregnancy, intended, mistimed and unwanted, might not fully describe a women's feelings about pregnancy, as moderately versus seriously mistimed could lead to different outcomes. They found a substantial range in mistiming, from 24 to 60 months, with pregnancies among younger women; never married women and 
African American women mistimed by significantly more months than other women.

Stress and challenges to self-esteem are known to be factors in infertility treatment, particularly if it is prolonged (Schneider \& Forthofer, 2005; McQuillan, Greil, White, \& Casey, 2003). Moreover, although a successful treatment outcome and subsequent award of a low risk pregnancy status may resolve or lessen stress, ongoing recognition of a precious pregnancy may result in an increase of protective health behaviours. Taylor (2000) discusses how women who had experienced infertility, and had become used to frequent scanning, found it unsettling to not have the same frequency of scans once the pregnancy was out of the gaze of the fertility specialist.

The wantedness or intendedness of the pregnancy appears to have an important relationship on health behaviour, in areas such as maternal infant birth weight, smoking and initiation of prenatal care, smoking and alcohol use (Altfeld, Handler, Burton, \& Berman, 1998). After adjusting for socio-demographic influences, Altfeld et al (1997) found that wanted pregnancies were associated with less cigarette and alcohol consumption; however, there was no difference in the uptake of first trimester care across the two groups, thus wantedness and sociodemographic characteristics were significant. There is substantial evidence that some pre-pregnancy health modifications are known to positively affect pregnancy outcomes. For instance, dietary folic acid supplementation commenced three months prior to conception is known to substantially reduce the likelihood of neural tube defects (Green, 2002). A-priori is most likely to be taken up by the women planning pregnancy. Negative pregnancy outcomes and unfavourable maternal behaviour and outcomes for mother and child are more common in unwanted pregnancies as opposed to mistimed (Speizer et al., 2004). An unplanned course is considered potentially more problematic to service providers, both from physical and social perspectives.

For nulliparous women, residual attitudes or feelings related to prior pregnancies may be an influential variable on the attitude toward the present pregnancy. The ease or difficulty experienced in falling pregnant, or prior experience of pregnancy loss, will have formed perceptions carried over into the present event (Armstrong \& Hutti, 1998; Stinson, Lasker, Lohmann \& Toedter, 1992). Each of these factors could influence a woman's psycho-social health, whether through reduced confidence, regret (for previous terminated pregnancies) or carrying a 'precious' 
pregnancy (Armstrong \& Hutti, 1998; Bergart, 2000; Stinson et al. (1992). A previous spontaneous abortion of pregnancy may alter their locus of control in the current pregnancy, eg., a greater reliance on external forces; both Powerful others and Fate or Chance; Wheatley, Brugha, \& Shapiro, 2003). Correspondingly, it may increase the tendency to make positive lifestyle changes.

\section{Risk status}

A woman may be classified as high risk for a number of reasons. Whilst a large proportion of high risk pregnancy may be attributed to a previous childbirth event, a nulliparous woman may be high risk due to a pre-existing medical condition or circumstances arising out of the present pregnancy. The experience of being high risk can be associated with increased contact with health systems, a need for increased vigilance and compliance to prescribed health actions, increased concern for self and the baby and a lack of an assured positive outcome, including a successful birth (Gupton, Heaman, \& Cheung, 2001).

This sub-section has discussed how the circumstances of the pregnancy impact on the way a woman may relate to the pregnancy. In particular, the concept of pregnancy intendedness is a complex construct and many studies have drawn a distinction between unwanted and mistimed pregnancies. Although a mistimed pregnancy is 'unplanned', it may not necessarily be unwanted. Infertility and risk were also discussed as factors that might be salient in a woman's perception of the pregnancy.

\subsubsection{DRIVERS OF TRANSITION}

Sawyer (1999) labelled active preparations and protection of self and baby in response to pregnancy commencing as 'engaged mothering', which may commence when a woman tries to become pregnant, becomes pregnant, has a pregnancy confirmed, or decides to continue the pregnancy. Initiating Lead Maternity Care can also be seen as a confirmation of preparation. Theoretically, women in New Zealand have choice in their Lead Maternity Carer. Also, the decision of whom to engage is dependent on what their desire and expectations are and the extent of their market intelligence and ability to access the LMC with the characteristics they seek. Taylor (2000) states; "when a woman plans and contracts for a particular kind of prenatal care, for a particular type of birth, she is (to the extent that such a thing is possible) engaging to purchase a particular kind of pregnancy experience" (p. 406). Therefore, the philosophies of the carer contracted will play an important role in the way the pregnancy is overseen and 
its management arranged. It is important that provider and client philosophies, of childbirth and pregnancy related goals, are in harmony.

\subsubsection{Information}

Information, involvement and referral to attitudes for cues (and/or the formation of context specific attitudes) are seen as key influences in adjustment and progression within the pregnant health lifestyle. Pregnancy is a novel experience for most nulliparous women, during which a number of significant personal, social and biological changes coincide (Smith, 1999). Women who have experienced a previous pregnancy that terminated in the first trimester will have some prior knowledge and experience, but this will not extend past the first few weeks of the current pregnancy (Smith, 1999).

\section{Information seeking activity}

Seeking information about pregnancy health is a key way of reducing an acknowledged gap in their knowledge resources and as a means of preparing for motherhood (McKenzie, 2001). It may be the greatest source of transition stimulus, given a lack of experience and existing referral cues. Gennaro and Fehrer (2000) found that pregnant women have a strong interest in information about pregnancy and the post partum.

Deutsch, Ruble, Fleming, Brooks-Gunn, and Stangor (1988) concluded that nulliparous women actively sought information and used it to construct identities incorporating motherhood as a way of self-socialisation. Dutta-Bergman (2003) examined general health consumers' intention to seek out health information beyond a doctor. Demographic variables that played a significant role were age (intent increasing with age), education (as a positive ingredient for a knowledge gap theory and information being sought to reduce the gap) and gender (women were more likely to seek out health information than males). The three psychographic variables of health consciousness, health consumerism and environmental consciousness accounted for most of the variation (cited in order of significance).

Information seeking leads to increased self-responsibility. Maher (2003) states that individual responsibility for health outcomes has been an increasingly prevalent aspect of public health discourse and the contemporary health experience in developed countries. The proliferation of health information (books and the Internet) has led to an increasing expectation of health surveillance. 
Maher (2003), presents an interesting conundrum whereby women are exercising their consumer rights in how they do [sic] pregnancy and birth, "they are in a position to challenge medical dominance as the informed and assertive patient" (p. 143). However, such structures also have the effect of reinforcing women's responsibilities for these outcomes; thus, they have reinforced attention to scientific and medical information as the best way to take this responsibility. The availability of information comes with an imperative to access and use the information; therefore, as stated by Maher (2003) : "for the women interviewed, the process of preparing for birth was very much seen as a research project purchasing and comparing books, taking all available pamphlets, listening to health professionals and being skeptical and informed consumers" (p. 143).

\section{Information sources}

Information related behaviour encompasses a range of activities including seeking, processing, evaluation of source usefulness, forming or referring to response outcome beliefs. Women can receive formal and informal information from numerous sources. Historically, health professionals have been a main source of information transfer to women. With a high level of source credibility and reliability, their information can be considered influential. However, today it competes with several other sources, including the Internet. Source credibility is an important influence in message persuasiveness and as pregnancy is often a new situation for women, information sources need to be determined for reliability and usefulness to them. The next few paragraphs provide an overview of the main channels through which information might be obtained.

McKenzie (2001) found that for pregnant women, a wide variety of information seeking practices existed in 'Every Day Information Seeking' (ELIS), made up of behaviours such as environmental scanning, chance encounters, lay referral and connecting by proxy. Critiques of this term, such as Erdelez (1999), believe that the label 'information seeking behaviour' is a misnomer because passive and opportunistic information seeking (such as environmental scanning or information 'encountering') resembles gathering more than hunting (as the term seeking implies). For some women, intuition or 'listening to one's body' is also an important source and guide in decision making.

Opportunities for vicarious learning about childbearing are thought to be reduced in today's Western societies, where pregnancy rates are declining and family structure makes it less likely for a woman to be living with or close to a pregnant 
family member. However, as the pregnancy progresses, knowledge accumulated from a bank of experiences will be available to the woman, some of which will remain relevant for the duration of the pregnancy (Deutsch, Ruble, Fleming, Brooks-Gunn \& Stangor, 1988).

Close friends and family are a source of information and usually very influential. Dunn, Pirie, and Hellerstedt (2003) found that $91 \%$ of women used a confidant (usually a mother) and compared it with advice given by the doctor. The doctor's advice was usually the most persuasive and then followed by the confidants. A pregnant woman's body can often be the stimulus for an acquaintance or relative stranger to offer advice. This can be welcome or unwelcome (McKenzie, 2001).

Books and health providers are the most important sources of advice (Aaronson, Mural, \& Pfoutz, 1988). Clissold, Hopkins, and Seddon (1991; cf. Dunne et al., 2003) found that few mothers reported that information, given to them by health professionals, influenced their behaviours during the pregnancy and puerperium. In fact, women in higher socio-economic status groups relied more on books and less on family and friends. The higher the level of perceived support from a provider, the more important they were as a source of information.

Since the late 1990's the role of the Internet in the provision of health information has become substantive (Lemire, Paré, Sicotte, \& Harvey, 2008). Pregnancy information sites, (including those operated by well known product brands), provide chat rooms for Internet users to converse with each other, as well as weekly baby developmental diaries or updates and pages that give medical information. Social networking also provides a wide opportunity to share experiences and interact, as do sites such as YouTube which provide opportunities to watch a growing bank of birth videos posted by women (Agazio \& Buckley, 2009).

Lifestyle and specialist magazines, such as Parents Centre and Cosmopolitan Guide to Pregnancy, discuss a wide range of topics; such as, nutrition and weight gain, safety in pregnancy, antenatal tests and healthcare. This medium, along with other media (Television, Internet, cinema), communicates information about celebrities and their behaviour in pregnancy, which can be persuasive and aspirational for some women through source attractiveness. Soap operas can deliver health messages, intended and unintended (Dutta-Bergman, 2006). Based on the results of an experiment, Dutta-Bergman (2006) found that, 
although soap operas were more likely to be viewed, and therefore 'reached', by younger women in lower socioeconomic groups (where poorer health statistics are generally found), they did not retain health messages as effectively as those in higher socio-economic groups.

Antenatal classes have been a part of maternity services for women for many years. In more recent years, it has become the norm to also involve partners. Specialisation, targeting particular segments (for instance ethnicity, youth) is now also a feature of the recognition and desire to service particular needs. Completion of an antenatal course could be seen as a 'rite of passage' for women, as it confers on them 'readiness' for labour. Studies promote some form of antenatal education as beneficial for women: it informs women on what to expect, can reduce fear and anxiety and bring about good antenatal self-care. By introducing women to a network in their community, it has a positive effect on support and may help establish enduring social networks into the postnatal period.

The type of media preferred pre-pregnancy will influence the type of information women have been exposed to. Vynkce (2002) recommended that media be included in psychographic studies as an important lifestyle factor. It is ideal that many of the pregnancy health messages reach women before pregnancy (for example, folic acid use), therefore it is useful to know what media is used and the preferences of different groupings. Pre-pregnant media can have an important attitude formation and socialisation effect (McKenzie, 2001). The media, particularly television, can exposes audiences to images of childbearing that are often dramatic and used to grow ratings. This can influence attitudes in women at a young age, as to how they view a future potential event. This 'learning' may well be drawn upon and influence impressions when the time comes.

\section{Strategies used to process information}

Responses to information, decision-making and choice selection are influenced by antecedent factors, and also pregnancy health beliefs and attitudes. Behaviour change models, such as The Health Belief Model (Risker, 1996), are useful in suggesting mechanisms by which decisions about change might be decided and acted upon. The processing ability of the woman is also dependent on cognitive functioning, ability to understand the 'language' information is transferred by, familiarity and comfort with the media source and their capability to work through potentially conflicting information received. Some may have a preference for an 
abundance of information which leads them through a complex evaluation of choices, whilst others would prefer reduction of complexity, pre-packaged advice and heuristic cues that reduce decision making effort. Root and Browner (2004) suggest that pregnancy is characterized by a "split subjectivity in which women straddle the authoritative and the subjugated, the objective and the subjective and the haptic as well as the optic" (p. 126), positing that pregnant women attempt to fuse the range of information by applying a broad spectrum of compliance and resistance to the many (often contradictory) bio-medically derived prenatal norms they encounter.

A positive response to information will involve a degree of planning and goal setting. How actively goals are pursued and the saliency of each goal depends on individual motivations and drive for accomplishment, access to resources, confidence in oneself and the meaning of not achieving the goal.

In conclusion, this sub-section has identified several variables influential in the information gathering, processing and subsequent response that occurs in pregnancy. Some of the information is actively sought whilst a significant amount is acquired more passively. A range of media is available to women, with much coming from reliable medical sources. However, the public press and Internet also provide several options for a woman to obtain information. Whilst the reliability and quality of information from public press and the Internet can be argued, these may be more readily digested sources than through formal channels.

\subsubsection{Pregnancy health attitudes}

Attitudes are important predictors of behaviour and are used in marketing as an indication of likely resultant 'purchase' behaviour in consumer situations, based on whether evaluation of attitude 'toward an object' is positive or negative (Bagozzi, 1992). Pregnancy attitudes provide a guide for the establishment of pregnancy health patterns by women, for instance, attitudes toward smoking, and alcohol intake in pregnancy (Godin, Valois, Lepage \& Desharnais, 1992). Many women have thought about the prospects of pregnancy in their life course and formed impressions at an early age, either through formal human development education in school, socialisation and exposure to primary experiences and/or media exposure (Larson, 1996). 
Indeed, it can be argued that a substantive amount of pregnancy relevant beliefs and attitudes might exist well before diagnosis of pregnancy, especially if the pregnancy is planned (Hellerstedt, Pirie, Lando, Curry, McBride, Grothaus, et al., 1998). Furthermore, a woman practicing high levels of pre-pregnant health promoting behaviours may well be expected to practice a high level of positive health behaviour in pregnancy (Hellerstedt et al.). In contrast, it may be the first time she has really thought about her health. Some women may find pregnancy a stimulus to engage in more concerted health management practices and behaviours that reduce risk (for example, cessation of smoking; Kost, Landry \& Darroch, 1998).

The tension between medical intervention and promotion of pregnancy as a natural event has been felt for many years (Stojanovic, 2008). According to proponents of non-intervention, medicalisation increases the perception of pregnancy as an illness. This leads to a legitimisation of technology as a necessary surveillance aid and that over observes (in low risk pregnancy) and helps to set up an intervention cascade, whereupon more and more observation and intervention becomes indicated (Stojanovic, 2008). However, much of the present cohort of women has grown up with technology as an everyday part of life (Tapscott, 1998), particularly with the increasing use of social networking through the Internet, which may well affect their attitudes to its use in pregnancy (Flanagin \& Metzger, 2001). Taylor (2000) discusses how sonograms (ultrasound), a widely used form of non-invasive prenatal testing, has come to offer benefits for the mother, along with medical screening and diagnostic information health providers seek. Increasingly, it has come to be a way for women to interact with their baby. By visualising their baby it provides an opportunity to 'see' the baby, bond with it emotionally, learn its sex, and be reassured it appears normal. A picture produced from the sonogram is tangible evidence of the baby which women can take home.

\subsubsection{Motivations and Barriers}

In order to make changes to maintain or practice the level of health promoting behaviour desired, perceived barriers must be addressed and constraints resolved. Many stimuli (both intrinsic and extrinsic) may be powerful motivators for a woman to pursue desired goals. Alternatively, barriers (internal and external) may be perceived as too restrictive to allow for positive sustained action (Harvey \& Faber, 1993). Factors that may lead to a low level of response can be poor self-efficacy, a perceived lack of support from others or physical conditions 
(for instance, fatigue and nausea). The SPARC (2003) "Obstacles to Action" study found (in a non-pregnant population) that external environmental factors, such as poor weather and fear of neighbourhood dogs, reduced outside activity. Fowles and Feucht (2004) assessed barriers to practicing healthy nutrition in pregnancy; they found access and cost (including transportation), unwillingness to give up enjoyable high fat foods and poor preparation resources (for example, 'the stove doesn't work where I live') to be significant factors. Lack of time can also be a constraint (SPARC).

One of the most important influences over a pregnant woman's lifestyle decisions and actions, is concern for the baby's present and future well-being (Rubin, 1987; Mercer, 2004). For a time, one human's present and future health is utterly and directly dependent on the behaviour of another. Considerations for the baby must be factored into assessment of maternal motivations and drivers of pregnancy health behaviours. Motivations and health attitudes generated by concerns for the self may be secondary to concern for the baby. This can be seen in behaviour such as avoidance by some women of simple pain relief or over-thecounter remedies (for example, Panadol) because of their suspicion that they may be dangerous for the developing baby (Dunlop, Gardiner, Shellhaas, Menard \& McDiarmid, 2008).

In summary, this sub-section has discussed the ways attitudes toward pregnancy health are formed. The onset of pregnancy heralds the start of forming these attitudes to a large extent. Motivations and barriers serve as enablers or detractors from practicing health in pregnancy. 


\subsubsection{PHYSICAL AND PSYCHO-SOCIAL DIMENSIONS OF PREGNANCY}

Pregnancy is as much a physical transition as a psycho-social one, with both domains being well discussed in the literature on pregnancy. Firstly, this section presents the physical behaviour dimensions from the literature identified as salient for this study (nutrition, exercise, sleep and rest, risk reduction and substance use). Attending physical check-ups regularly, included in many health measurement frameworks as evidence of health-promoting behaviour, is noted but not included in the study design, as participants were identified through their healthcare provider and assumed to be in regular contact. Discussion of psychosocial aspects of pregnancy within the literature is substantial and contributed to by several disciplines. The psycho-social dimensions identified in the literature as significant to fetal/maternal outcomes and positive transition into the post partum period are the pregnant self image, support networks, emotional changes and coping and rest and relaxation activities.

\subsubsection{Physical dimensions}

Major physical dimensions in pregnancy health are similar to those that make up health lifestyle generally. Additionally, however, there are some needs that are unique to pregnancy (such as recommendations for alcohol cessation to maximize positive outcomes) and these needs are discussed below.

\section{Nutrition}

Nutrition in pregnancy generally covers the concepts of maternal weight gain and nutritional intake (Chomitz et al., 1995). A third area, less discussed in the literature, but becoming increasingly relevant, is the need for specific food avoidance and how interruptions to normal eating patterns are dealt with. Kaiser and Allen (2008) recommend good nutritional status through "a lifestyle that optimises maternal health and reduces risks of birth defects, sub-optimal fetal growth and development and chronic health problems in their children" (p. 553). Good nutrition is key to increasing the likelihood of positive outcomes for mother and baby (Kaiser \& Allen, 2008).

During pregnancy, nutritional needs alter, with increased requirements for calories and nutrients (particularly protein, iron, folate and other B vitamins) to meet the requirement of both the fetus and expanding maternal tissues (Chomitz et al., 1995). There is an emerging body of evidence that suggests the in-utero environment may be responsible for long term effects, such as some childhood illnesses and conditions. These effects include atopy (Dean et al., 2007) and may 
extend to disease in later life such as obesity, diabetes and heart disease (Brown, Murtaugh, Jacobs, \& Margellos, 2002; Vickers et. al, 2003; De Boo \& Harding, 2006; Revington, 2004; Gluckman \& Hanson, 2004).

Poor nutrition may be due to a number of factors: demographic risk (for example, adolescence), voluntary restrict intake through dieting or calorie restriction, insufficient food budget, unbalanced diet, poor nutritional knowledge, cooking skills or equipment (Chomitz et al., 1995). Several clinical studies have been conducted that consistently link prenatal weight gain with birth weight. Low maternal weight gain and poor quality of nutrition is a major factor in low infant birth weight or being 'small for dates', a condition correlated with a poorer health prognosis (Ashdown-Lambert, 2005; Brown et al., 2002; Fowles \& Feucht, 2004). Factors apparent in women most likely to have poor weight gain and poor nutritional intake are lower income and associated other unhealthy behaviours, such as smoking (Ashdown-Lambert, 2005; Brown et al., 2002; Fowles \& Feucht, 2004).

The benefits of vitamin supplementation such as folic acid to reduce the incidence of neural tube defects is now well established (Green, 2002; De Walle \& De Jongvan den Berg, 2007. Numerous studies have been conducted on dissemination of information and reported levels of knowledge along with motivation and compliance with recommendations and guidelines (Green, 2002; De Walle and De Jong-van den Berg, 2007). Folic acid supplementation can also be viewed as at risk reduction health behaviour, as well as a nutritional behaviour. The recommendation of a therapeutic intake of folic acid three months prior to conception and in the first trimester has been proven to drastically reduce neural tube defects (Langley-Evans \& Langley-Evans, 2002); albeit, only likely to occur if a woman is planning a pregnancy in the near future. Langley-Evans and LangleyEvans (2002) surveyed 300 pregnant British women and found $67 \%$ consumed folate containing supplements in the first trimester of pregnancy and $43 \%$ reported taking folate before becoming pregnant. Non-compliance amongst this sample was reported most commonly in those under the age of 21 years, in smokers and those from lower socio-economic groups. Knudsen et al. (2004) found lower compliance with folate recommendations in Danish women planning pregnancy, even following an information campaign the previous year. Only $22 \%$ of women interviewed had followed that full recommendations for periconceptional folic acid. Young age, low education and smoking were identified as factors that determined non-compliance. One of the recommendations coming 
from both of these studies is that food be fortified with folic acid, not only in view of the incidence of unplanned pregnancy, but also because of relatively low compliance rates. It can then be assumed that the timely intake of folic acid is an important positive health behaviour in pregnancy.

In the early weeks of pregnancy, barriers to healthy eating are often attributed to problems with nausea and vomiting or disinterest in food preparation due to lack of appetite and nausea (Quinla \& Hill, 2003). As the pregnancy develops, cravings or 'pica' can also challenge optimal eating patterns and food choices (Cooksey, 1995; Mikkelsen, Andersen \& Olsen, 2006). More recently, features of pregnancy have become the need to avoid foods or food handling practices that might lead to diseases such as toxoplasmosis or listeria (Ross, Jones, \& Lynch, 2006; Bondarianzadeh, 2007; Delgado, 2008; Antoniou et al., 2007) and ingestion of foods contaminated by substances such as mercury (Xue, Holzman, Rahbar, Trosko \& Fischer, 2007). There are no studies reporting the effects of food avoidance on lifestyle or levels of compliance.

\section{Exercise, sleep and rest patterns}

Exercise in the health literature focuses on the nature and amount of exercise considered to be beneficial, women's beliefs about exercise in pregnancy, changes to normal patterns of exercise, the types of exercise engaged in and barriers to exercise. Moderate exercise in pregnancy is believed to be very beneficial for both mother and baby (Chomitz et al., 1995).

Clarke and Gross (2004) studied women's behaviour, beliefs and information sources about physical exercise in pregnancy and found that physical activity was reduced during pregnancy due to physical changes and a combination of social and psychological processes. Rest and relaxation were perceived as being significantly more important during pregnancy than regular exercise or the maintenance of an active lifestyle. Participants who reported receiving advice about physical activity at least once in pregnancy were significantly older, more educated and of a higher activity level pre-pregnancy. Evenson, Savitz and Huston (2004) examined leisure time physical activity amongst non-pregnant versus pregnant women during the previous month, in order to assess prevalence of and characteristics associated with participation. They found the most common leisure activity for pregnant women was walking, followed by activities such as swimming, weight lifting, gardening and aerobics. The most common exercise beliefs during the post partum were that exercise controls weight gain 
and a lack of time obstructed exercise participation. Symons and Hausenblas (2004) found the most common exercise beliefs during pregnancy were that exercise improves mood and that physical limitations (for example, nausea) obstructed exercise participation. Additionally, a woman's partner and family members most strongly influenced their pregnancy and post partum exercise behaviour and women exercised more before they were pregnant than during pregnancy and post partum.

Among pregnant women, any physical leisure activity was significantly higher for those with higher education, younger age and excellent or very good health. Pregnant women reported less leisure activity than non-pregnant women of the same age (Evenson, Savitz \& Huston, 2004). Symon-Downs and Hausenblas (2003) examined women's exercise intention and behaviour from their second to third pregnancy trimester using The Theory of Planned Behaviour (TPB), with self reporting on the dimensions of their exercise attitude, subjective norm, perceived behavioural control, intention, and behaviour. They found that attitude was the strongest determinant of exercise intention, followed by perceived behavioural control, and subjective norm.

Sleep and rest patterns can be significantly disturbed in pregnancy, particularly in the last few weeks. Sleep problems most commonly reported are restlessness, waking frequently to go to the toilet, leg cramps and 'restless legs syndrome' (Kirsch \& Avidan, 2005; Tunc, Karadag, Dogulu, \& Inan, 2007; Hensley, 2009).

\section{Risk reduction}

Many theories of transition to motherhood encompass a woman's efforts to reduce risk to ensure the safety of herself and the baby. Rubin (1967) defines this as 'ensuring safe passage', whereby a woman will engage in protective behaviours, aimed at eliminating controllable threats. Heaman-Gupton and Gregory (2004) explored perceptions of risk amongst pregnant women, in which women described factors they considered in making personal risk assessments. Themes that emerged were related to self image, history, healthcare and the unknown. Higher risk women voiced greater risk perceptions and specific risks, whilst low risk women raised perceptions of risk that were diffuse and hypothetical. Risk reduction can involve numerous activities: such as, obtaining healthcare and avoidance of potentially dangerous substances (alcohol and smoking, including passive smoking). The avoidance of environmental danger 
may include avoiding physical harm, trips and falls, domestic violence, or the wearing of safety equipment (seat belts) (Mercer, 2004, Rubin, 1987).

\section{Screening tests}

Avoidance of harm from both the real and 'unknown' may lead a woman to undertake screening and tests that are invasive or non-invasive. Women may also decline recommended tests if the fear potential risk to the baby (Liamputtong et al., 2003). The avoidance of risky foods and folic acid supplementation (discussed above) may also be considered as risk reduction behaviours.

\section{Dental hygiene}

Dental hygiene is considered an important aspect of prenatal care and might not usually be included in a framework for measurement of a health lifestyle. It is important due to the hormonal effects of oestrogen in softening gum tissue and complications arising from this, such as pre-term birth (Scannapieco, Bush \& Paju, 2003). Lydon-Rochelle, Krakowiak, Hujoel, and Peters (2004) found that $58 \%$ of pregnant women had no dental care during pregnancy. Of the group who reported no problems or did not attend a dentist, they were more likely to smoke, be obese and receive no dental counselling. Whilst recommendations would be to visit a dentist at least once during the pregnancy, barriers of cost, and time could be perceived as significant.

\section{Violence}

Partner violence toward a pregnant partner is a common environmental risk facing women. Chomitz et al., (1995) report that depending on the population surveyed, domestic violence is estimated to be between 8 and $17 \%$ (US figures). Violence is potentially dangerous for the victim (including life-threatening injuries, placental abruption); it can lead to intra-uterine growth retardation (IUGR) and subsequent low birth weight through various mechanisms, such as a partner's demand that the woman not gain weight (Campbell, 2001). Moreover, violence can cause 'battered syndrome', resulting in behaviour such as delayed seeking of prenatal care. Abuse during pregnancy is a complex problem and puts women at risk for a variety of physical and mental problems (Campbell, 2001). For instance, the more women are abused, the more likely they are to smoke, eat poorly and gain less weight (Campbell). 


\section{Substances}

Smoking, alcohol and drug abuse are three important factors viewed to have detrimental health effects on the developing fetus and potentially continuing impact post birth. The avoidance or reduction of these in pregnancy is an important risk reduction behaviour that a women theoretically has control over. Several studies (see for instance Chomitz et al., 1995; Butler, Williams, Paterson, \& Tukuitonga, 2004; Dejin-Karlson \& Ostergren, 2003; Stewart \& Streiner, 1994) have been conducted in these areas, not just on fetal outcomes but also on the lifestyles, motivations and attitudes of the mothers concerned.

Smoking is a major risk issue in pregnancy and its detrimental effects to the health of the developing fetus have been known since the 1970's (Chomitz et al., 1995) because of its associations with low birth weight, intra-uterine growth retardation and other negative outcomes. Dejin-Karlson and Ostergren (2003) found smoking in pregnancy appeared to be associated with socioeconomic, demographic and clinical risk factors. In smokers, there appeared to be substantial clustering of risk factors. Haslam and Lawrence (2004) found that pregnant smokers were less likely to increase folic acid intake, take vitamin and iron supplements and feel personally responsible for the health of the fetus. The authors suggested that infants of smokers might be placed at an intrauterine disadvantage, not only due to smoking, but also poor nutrition. Smoking, and its links to other problems, is also supported by a New Zealand study of smoking within a pregnant Pacific Island sample (Butler, Williams, Paterson, \& Tukuitonga, 2004). The study found that approximately, one quarter of the women smoked during pregnancy and smoking was significantly associated with several factors, including indicators of disadvantage and degree of Westernisation.

Excessive alcohol consumption in pregnancy is linked to congenital problems described as Fetal Alcohol Syndrome. The safety of alcohol intake in pregnancy remains controversial and recommendations vary from country to country. The New Zealand Ministry of Health (2006) recommends no alcohol in pregnancy. Alcohol intake in pregnancy has significantly decreased amongst higher educated women in the past decades; however the same rate of reduction has not been demonstrated in lower socio-economic groups (Chomitz et al., 1995). Stewart and Streiner (1994) examined alcohol drinking in pregnancy to determine the prevalence of regular drinking during the second half of pregnancy, the attributes that differentiate drinkers from non-drinkers and what characterises prenatal patients who drink seven or fewer standard drinks per week and those who drink 
more than seven. The seven plus a week drinkers were more likely to be under 21 years of age, poorly educated, unemployed, unmarried, to have unplanned pregnancies, emotional problems, eat unhealthy diets, smoke, use illicit drugs, and be physically abused. It was also found that these drinkers were more likely to 'meet psychiatric case status' and to believe that chance rather than 'internal control' affected the health of their fetuses as measured by the Fetal Health Locus of Control (FHLOC). However, contrary to these findings, Morris, Swasy and Mazis (1994) found in a study of women who drink in pregnancy, that they tended to be older, had less trouble becoming pregnant than abstainers and viewed alcohol in pregnancy as less risky than others. They reported: "this segment of women who drink appeared to have a more sophisticated approach to 'understanding' or 'rationalising' the risks of alcohol for the unborn child" ( $p$. 142).

The use of illicit or illegal drugs in pregnancy is an important issue not just because it is likely to co-exist with other risky health behaviours, but because the fetus will also suffer addiction, from which withdrawal will occur following birth (Chomitz et al., 1995). However, the collection of information on any type of substance abuse, or other behaviour that has a stigma effect using self-report, is potentially problematic (Chomitz et al.). Therefore, such information may not be easily gained on enquiry.

\subsubsection{Psycho-social}

The psycho-social dimension is a key dimension of holistic health. Positive adaptive psycho-social changes occurring in pregnancy make a key contribution to a successful transition.

\section{Body image}

The 'pregnant self image' is a woman's feelings and impressions of herself during pregnancy. This self image is shaped by pre-pregnant self-image, beliefs about pregnancy and others' attitudes and responses toward the pregnant woman. A positive self image in pregnancy can lead to increased enjoyment and coping and appears to have a positive influence and association with other aspects of health (Earle, 2003).

There is little empirical work on self image in pregnancy and it is often assumed that the usual objectification and comodification of feminine attractiveness does not extend to pregnant women (Earle, 2003). While it is generally believed that 
pregnancy allows the woman to step outside the 'tyranny of slimness', Earle (2003) argues against this, suggesting the opposite may be true; that is, how a woman looks during pregnancy is important, and women believe pregnancy is not an opportunity but a temporary state. Some women may believe that changes to their physical appearance are irreversible (for instance, stretch marks) and they view pregnancy as a time when physical appearance is irrevocably altered for the worse. Clark and Ogden (1999) developed a theme of relaxed boundaries around the body, finding women complained that other people felt able to touch their pregnant body without regard for the normal respected boundaries. Whilst for some women this attention may be accepted or welcomed, for others it may be unwelcome and increase their feelings of being somewhat of a spectacle (Clark \& Ogden, 1999).

The concept of the 'wayward' body has been applied to pregnancy, with gestation likened to a specific body episode which belies the modern Western conviction that one's body can be possessed and molded accordingly (Warren, 2004). Some may invest in 'the body project', constituted by the efforts to sculpt their bodies in culturally acceptable ways. However, research findings are contradictory (Johnson, Burrows, \& Williamson, 2004). Some authors argue that pregnancy represents the greatest deviation from a woman's ideal body image (Strang \& Sullivan, 1985) and that as the pregnancy progresses, women become more negative about their appearance (Drake, Verhulst, Fawcett, \& Barger, 1988; Strang \& Sullivan, 1985). However, alternative research suggests that pregnant women are less dissatisfied with their bodies than non-pregnant women (Clark \& Ogden, 1999). Devine et al. (2000) contend that weight gain in pregnancy is for some distressing, for others liberating; for some, a feeling of loss of control is unpleasant, whilst for others it leads to great enjoyment, causing them to "Iuxuriate in their materiality" (p. 202). In the marketing of products and ideas, the use of attractive models and celebrities as important sources is well established and it is conceivable that the same mechanism may operate during pregnancy. Numerous actresses and role models have been featured pregnant in lifestyle magazines over recent years (Longhurst, 2005. Celebrities may also influence other women in regards to their 'control' over their body; for instance, many celebrities, such as Heidi Klum and Victoria Beckhem appear to regain their pre-pregnant body shape quickly after birth. 


\section{Support}

Social support influences a person's sense of well being. Social network members, through which support is drawn, can have a negative or positive impact or influence (Rubin, 1987). Authors such as Rubin (1987) discuss the need for ongoing support and acceptance of the pregnancy and fetus by a woman's support network with seeking the support of others as an important factor in achieving the goals of the transitional tasks of pregnancy. Social support can influence engagement in health promoting behaviours through its role as a modifying factor, with growing literature to suggest that social relationships have a positive impact on physical health and psychological wellbeing (Feldman, Dunkel-Schetter, Sandman, \& Wadhaw, 2000; Dejin-Karlson \& Ostergren, 2003). Social support has been proposed as an influence on infant birth weight, with poorer support associated with 'small for gestational age' (SGA) babies (Feldman et al., 2000) and may also mediate other behaviours such as smoking and alcohol consumption (Dejin-Karlson \& Ostergren, 2003).

As early pregnancy often causes an alteration to a person's perceptions of wellbeing and usual state, family or household members are often sought for support, both emotionally and practically (for instance, food preparation during morning sickness) (Florian \& Mikulincer, 1995). Important others are often drawn into decision making, particularly in the selection of locality and preparation for birth. Increasingly, many households consider changes in the planning process well prior to conception. At this time the quality of primary relationships is important (Florian \& Mikulincer, 1995; Lederman, 1996).

As well as acceptance and support from the 'most significant other' (usually the woman's partner), friends and extended family provide support and can be an important source of information. The woman's relationship with her mother has been studied by a number of authors who describe first pregnancy as a social as well as physical event, where a mother's support is often sought. However, it is also a time when a woman's own mothering is re-examined and evaluated, potentially leading to a consolidation of one's relationship with their mother, and more equality, where they seek to draw closer and internally reconcile with their mother (Walzer, 1995,; Valentine, 1982 in Walzer, 1995). Lederman (1996) found that women who had more conflict in relationships with their mothers experienced difficulty and a mother who is critical, unsupportive, or unavailable may undermine a woman's confidence. For some women, the advice of a mother may be welcomed as affirmation of involvement and interest, but not necessarily 
regarded as credible. A friend who has recently had a baby may play a relatively more important role as an information source.

Pregnancy as a precursor to a new life stage may activate the seeking out of new contacts and lead to formation of relationships with those in a similar stage (Murphy Tighe, 2010). Antenatal contact through pregnancy classes or through mediums such as the Internet and discussion boards may start this process, with contact continued into the post partum in an effort to reduce potential feelings of isolation as women adjust to a new role of motherhood (Murphy Tighe). For some, this represents the first extended period of time at home and away from paid employment.

\section{Stress and coping}

Many theorists view pregnancy from a stress, or crisis perspective (Bondas \& Eriksson, 2001) and stress is regarded as playing a major part in determining physical health and illness (Selye, 1950; Cassidy, 2000). Concerns over safety, new behaviours for nutrition and exercise, and taking over a nurturing role can place affective demands on attention (Stark, 2001). An excessive amount of stress and anxiety may increase the risk of a SGA baby due to stress hormones. Women who report higher levels of social support have less circulating levels of stress hormones (ACTH and cortisol) (Dejin-Karlson \& Ostergren, 2003. In a study of women who feared vaginal birth, Salmela-Aro, Nurmi and Halmesmaki (2001) found that anxiety, neuroticism, vulnerability, poor partner relationship and lack of social support influenced the level of fear and affected a woman's attitude to the approaching delivery. Walker, Cooney, and Riggs (1999) explored the relationship of psycho-social and demographic variables to health behaviours in early pregnancy, with results showing that higher depressive symptoms, lower internal locus of control for fetal health and lower family income were related to poorer health behaviours in the first trimester of pregnancy.

Huizink, Robies de Medina, Mulder, Visser, \& Buitelaar (2002) state that significant research has been undertaken on high risk women (eg., substance abusers and adolescents) and coping (often through avoidance and aggressive responses). Little is known, however, about coping in nulliparous low risk women, who in the main are exposed to an average level of stress throughout pregnancy. These authors looked at the occurrence of emotionally focused problem coping, problem focused coping (planning, information seeking and finding solutions) and avoidance (coping through distraction activities or seeking 
social diversion). They found that although respondents felt that problem focused coping was more effective, they sought social support more, particularly if the situation is considered uncontrollable. As a strategy to cope in pregnancy, decisions that are rationally or emotionally based may be used; emotion focused coping and problem focused coping may be employed (Huizink et al., 2002). The ability to relax and use recreational activities as a stress reduction tool is an important health promoting behaviour in most health lifestyle frameworks. As women may find that their usual stress reduction activities are not appropriate for pregnancy (physical sport, alcohol), they will need to find new but effective activities for the pregnancy.

In summary, this sub-section has investigated the important physical and social aspects of pregnancy health and how numerous and complex strands weave together to form the health lifestyle of a woman. Each of the aspects discussed are relevant for this study, as they are determinant of the pregnancy health and/or pregnancy outcomes.

\subsubsection{TRANSITION PROGRESS AND OUTCOMES}

A positive transitional health lifestyle is accumulative in terms of experience and knowledge growth and progressive in that it moves in a forward direction from one point to another, where one arrives transformed and ready for the tasks at hand. It demonstrates adequate role mastery of the tasks at hand (attachment to baby and positive anticipation of a new life stage; Rubin 1987; Mercer 2004). A healthy response as pregnancy progresses theoretically leads to healthy outcomes in the post natal period and possibly a reduction in the risk of postnatal depression.

The non-static nature of transition adds to the complexity of evaluating transition in non-longitudinal studies or snap shot approaches; even a simple linear explanation of transition movement may, in itself, not do justice to complex health adaptations. Even though physical development in pregnancy is linear, some authors believe that psycho-social development progresses in a spiraling or widening manner (Kegan, 1982; Rubin, 1984). This suggests the possibility of 'cycling' or 'rhythms' throughout the term of the transitional lifestyle and the need to remain open to non-linear effects over time.

The transition from childlessness to motherhood requires many changes, which can lead to conflict. Lederman (1996) and Smith (1999) discuss possible selves 
that may emerge from pregnancy, as a result of identity development during transition to motherhood. The transition period of pregnancy can be potentially disorganising or a developmental opportunity and an opportunity to grow or regress.

\subsubsection{Transition markers}

The works of Rubin (1987), Mercer (2004), and Schumacher and Meleis (1994) present a picture of what the markers of successful transition in pregnancy would comprise; this includes factors such as maternal/fetal attachment, perceptions of wellness and a readiness to enter a new life stage.

Emotional attachment to the baby is an important concept, as it indicates positive acceptance and acknowledgement that the pregnancy is producing an infant, or person in their own right (Schumacher \& Meleis, 1994). Behaviours that indicate emotional attachment are anticipation of motherhood, excitement about the baby, getting things 'ready for baby', talking or singing to the baby and fanaticizing about the baby (Schumacher \& Meleis, 1994). A low level of attachment can signal potential problems in the post-natal phase and an increased risk of post-natal depression (Priel \& Besser, 2001. Maternal-fetal attachment scales have been developed by several authors; such as Müller's Maternal Attachment Inventory (1994) and Condon \& Corkindale's Maternal Antenatal Attachment Scales (1997), measuring quality of and pre-occupation with attachment to the unborn baby (Priel \& Besser, 2001).

A general sense of wellbeing reflection and one that encompasses both physical and psycho-social considerations can be a positive indication that a positive transition is being experienced (Wallace, et al., 2009). Schumacher and Meleis (1994) believe successful transition occurs when feelings of distress or disorganization are replaced with feelings of wellness.

Anticipation of motherhood and a 'new normal' requires a new self identity, which is formed during the transitional period of pregnancy (Mercer, 2004). The change from being without children, to having responsibility for an infant, is without doubt one that requires significant adjustment and role change. This can be accompanied by grief for the roles given up that will not be compatible with a new life stage. The process of maternal self-socialization made during pregnancy involves creating a new self-image (that is, as a mother), seeking out new networks, leaving old ways behind, practicing and testing new roles, and planning 
for a new 'normal' (Deutsch, Ruble, Fleming, Brooks-Gunn, \& Stangor, 1988). These types of perspectives reinforce the value of using a transitional lifestyle framework as the integrating idea for this study. It is holistic, yet allows for multiple dimensions to capture the richness and complexity of the phenomenon under investigation.

Preparation for a new normal commences with recognition that the pregnancy period will end with a birth event. The timing and extent to which a woman plans and engages in goal setting and attainment is dependent on many factors and considerations: resources, information available, ability to confront fears and anxieties and prepare for the inevitable, and the degree to which the woman is autonomous, or works with her partner or care provider (Mercer, 2004; Rubin, 1987; Schumacher \& Meleis, 1994). Recognition and realization of the approaching new life stage with a positive anticipation and with relationships intact is a signal of successful transition (Schumacher \& Meleis, 1994). Keeping mementos, memory markers or souvenirs (CTG strip, scan photographs, 'belly casting'), keeping a diary (for self or others) and celebrating rites of passage (notably the baby shower) demonstrate both the valuing of pregnancy and acknowledgement that the life stage will end (Schumacher \& Meleis, 1994.

\subsubsection{A TEMPORARY OR TRANSFORMED LIFESTYLE?}

If a woman departs from pregnancy into motherhood successfully, there is no doubt that a degree of transformation will have occurred during the pregnancy transition period that has enabled her to make this life course transit (Rubin, 1987; Mercer, 2004). In consideration of this, authors suggest that this period may well be a fertile time for permanent health behaviour change messages, as women are clearly reactively engaged and involved in their health (Smith, 1999). Smith found that after birth, the changes that occur in pregnancy can contribute to subsequent transformation of the woman's priorities and life options:

After birth, as women adjust to the presence of a new child, attention turns again to their own life projects. The process of pregnancy and becoming a mother can facilitate a transformation in these plans. The changing view of work during pregnancy, together with the growing involvement with key others, may help effect a longer term shift in the woman's perceptions and priorities. (p. 294) 
Some health behaviours that are known to be detrimental to health (smoking and excessive alcohol use) are often stopped or at least minimized during the pregnancy and a long term application would indeed be beneficial (Gennaro \& Fehrer, 2000). However, there is some evidence that changes in pregnancy might not carry over into a new life stage, but have been made only for the period of the pregnancy. For instance, in regard to smoking cessation, once the pregnancy is over many women relapse (Chomitz et al., 1995), with as many as $80 \%$ of those who stopped smoking in pregnancy restarting in the first year postpartum (Chomitz et al., 1995; Gennaro \& Fehrer, 2000).

Devine et al. (2000) also examined whether behaviour changed much over a life course trajectory. They examined weight concerns and weight management as life course trajectories in the transition to motherhood and the strategies women use to deal with weight changes (such as exercise and dieting). They found four main segments: relaxed maintenance, exercisers, determined and unhurried. Post pregnancy orientations to body weight changes remained the same as prepregnancy orientations, indicating that there may be a resumption of usual patterns of health behaviour in the post partum, thus giving support to a life course trajectories proposition. These findings reinforce further the adoption of a transitional lifestyle framework, which allows for significant permanent change in many health related behaviours and the possibility of reverting back to previous (sometimes adverse) behaviour post partum.

\subsubsection{SECTION SUMMARY}

This section has comprehensively explored the literature for physical and psychosocial dimensions of pregnancy health. Both dimensions are perceived as equally contributory to an understanding of holistic pregnancy health, and particularly helpful to a health marketing/consumer behaviour frame. Although the usual way to assess pregnancy health outcomes would be an assessment of the condition of mother and baby at home, and growth and development of the dyad in the early weeks post-partum, for the purposes of this study, alternative transition markers have been discussed and chosen. These markers are attachment, a general sense of wellbeing and preparation for a new normal. This section also discussed the extent to which health changes in pregnancy would be continued post partum. Some authors feel that the process of becoming a mother can lead to changes in some priorities and a transformation in some areas. However, for some there is evidence that some behaviours given up for the pregnancy (for example, smoking) may be resumed post partum. 


\subsection{CHAPTER 2 SUMMARY}

Bringing together the numerous factors that underscore the transitional health lifestyle of pregnancy results in a relatively complex discussion where several existing bodies of knowledge and ideas must be woven together so that interactions can be explored. An understanding of concepts such as lifecycle, lifestyle, transition and health are essential so that their combined contribution to a study definition of transition lifestyle and health lifestyle can be applied to pregnancy.

The sub-disciplines of marketing, healthcare marketing and social marketing contribute to the marketing relationship that is active in the study context. Marketers and health professionals play out both unique and dual roles in the part of provider/consumer interactions. The role of marketing is not only to assist in the delivery of quality service delivery but also to inform, design and implement behaviour change campaigns alongside health communicators who work in clinical settings.

In the review, the study context of pregnancy was discussed. Historic and socially embedded beliefs and traditions, that have been important influences on contemporary health services, are described. The increasing role of health consumerism and empowerment means that pregnant women have the opportunity of a more active role in the health course of their pregnancy. The history of maternity care in New Zealand is one where different views and ideologies have led to tensions between stakeholder groups. Today pregnant consumers theoretically have choice, but yet this leads to responsibility and an expectation that they manage their health in an informed fashion.

Examining the health lifestyle of pregnancy from a comprehensive and holistic approach revealed the presence of numerous studies from multiple disciplines, in particular those that focus on the physical and psycho-social dimensions of pregnancy health. The goals of each of these are similar in that they aim to better understand the role of context, motivations, values and attitudes of individual and groups and responses to the physical and psycho-social changes pregnancy brings. A large portion of these studies are designed to identify problem behaviour so that ultimately intervention will lead to a reduction in poorer pregnancy outcomes. The context of low risk pregnancy has been less 
studied, but works in this area are helpful in illuminating the transition course of 'normal' pregnancy.

This literature review has identified a significant gap in the literature that describes the transitional lifestyle holistically from a health perspective and within the approach of a marketing understanding of lifestyle segmentation. By applying marketing segmentation variables together with more traditional demographic, health diagnostic and socio-cultural variables (for example, life stage transitions), a greater understanding of the role of lifestyle in the course of pregnancy health can be offered.

The extensive review of components of pregnancy health style enables a guiding conceptual framework to be devised to enable an exploratory investigation to be conducted. This framework is explained in the next chapter. 


\section{CHAPTER 3. CONCEPTUAL FRAMEWORK AND RESEARCH QUESTIONS}

\subsection{CHAPTER INTRODUCTION}

This chapter provides the author's conceptualisation of the Transitional Pregnancy Health Lifestyle, based on the literature. Firstly, the main understandings applied to the development of the framework are outlined, and the conceptual framework is presented diagrammatically. An overview of each major dimension is then provided, followed by a specification of the full set of variables that underlies each dimension. This includes some discussion of rationale for variable inclusion, and the main theoretical work from which they are derived.

The conceptual framework does not provide a formal model for testing, although the specific measurement categories are carefully specified. The research questions are therefore not posed as formal hypotheses, but the full variable set was analysed to explore and characterise the pregnancy lifestyle as a basis for segmentation using a two-stage approach to cluster analysis.

\subsection{BACKGROUND}

The literature review examined and synthesised findings and research approaches relevant to the maternity consumer context, the marketing/provider relationship within that context and health psychographics. The literature review then drew together the multiple facets of what would make up a pregnancy health lifestyle and one considered to be in transition. The idea of transition success, as made up of a collective of health promoting psychosocial factors that demonstrate positive transition, was also explored in order to determine the extent to which that could be determined prior to the birth of the baby. Finally, the concept of segmentation profiling and interventions targeting specific groups was discussed. Although a number of theories informed the dimensions of health behaviour chosen in this study, Pender's Health Promoting Lifestyle II (1987) was notably influential in choosing the specific sub-domains used to operationalise the conceptual framework, as it provided a comprehensive set of attributes and behaviours that have been tested in both general health contexts and situation specific, including pregnancy. Four main theoretical ideas, grounded in the literature, underpinned the conceptual framework: Psychographics, Transition Theory, The Precaution Adoption Process Model and the Consumer Decision 
Making Process. Their influence can be seen in the selection and organization of variables within the overall conceptual framework.

\subsection{THE TRANSITIONAL PREGNANCY HEALTH LIFESTYLE CONCEPTUAL FRAMEWORK}

The conceptual framework at its broadest level is made up of three dimensions that are sequential in time: Influencing Lifestyle Factors, Life in Transition and Adaptation. Each dimension in turn contains sub-domains and sub-domain constructs and attributes operationalised as discreet groups of variables.

The conceptual framework is presented graphically in Figure 3.1, showing the framework dimensions and sub-domains. Table 3.2 summarises the dimensions, sub-domains, constructs/attributes and variables of the conceptual framework. A detailed discussion of each dimension, sub-domain, construct and attribute set and the variables assigned to the constructs/ attribute sets follows Table 3.2.

FIGURE 3.1. CONCEPTUAL FRAMEWORK: THE PREGNANCY HEALTH (PREGHEALTH) LIFESTYLE

Influencing Lifestyle Factors Life in Transition Adaptation

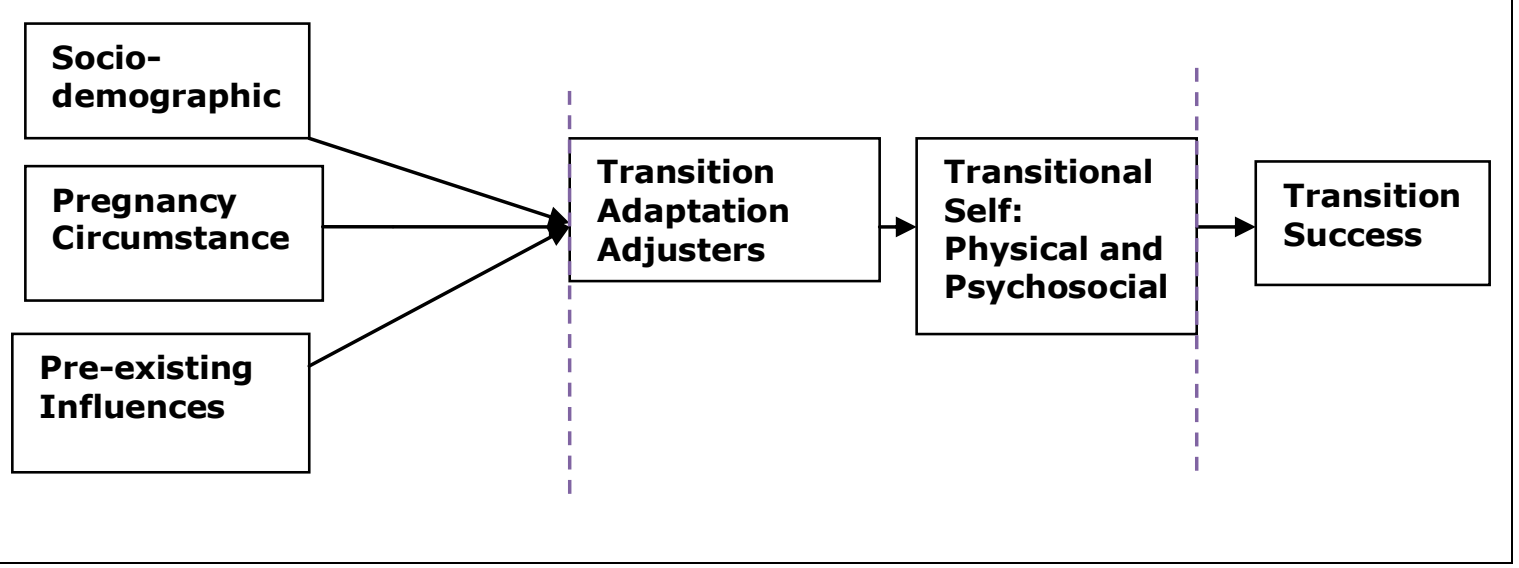


TABLE 3.2. CONCEPTUAL FRAMEWORK:

DIMENSIONS, SUB-DOMAINS, CONSTRUCTS AND ATTRIBUTES AND VARIABLES

\begin{tabular}{|c|c|c|c|}
\hline Dimension & Sub- domain & Constructs and Attributes & Variable/s \\
\hline \multirow[t]{7}{*}{$\begin{array}{l}\text { 3.4.1. Influencing } \\
\text { Lifestyle Factors }\end{array}$} & 3.4.1.1. Socio-demographic & Socio-demographics Factors & $\begin{array}{l}\text { Age, Ethnicity, Household income, Occupation, Employment, Education, } \\
\text { Living situation, Domicile, Media use }\end{array}$ \\
\hline & $\begin{array}{l}\text { 3.4.1.2. Pregnancy } \\
\text { Circumstance }\end{array}$ & Feelings about the Pregnancy & $\begin{array}{l}\text { Pregnancy intendedness, Original intentions for the pregnancy, Evaluation } \\
\text { of pregnancy worth, Rating as a life event }\end{array}$ \\
\hline & & Pregnancy Dates & Weeks now, First awareness \\
\hline & & Other Experiential Factors & $\begin{array}{l}\text { Level of risk, Knowing sex of baby, Previously pregnant, Fertility assistance } \\
\text { required }\end{array}$ \\
\hline & 3.4.1.3. Pre-existing Influences & Pre-existing Health & Health as a value, Health consumerism, Pre-pregnancy health level \\
\hline & & Mastery and Achievement & Sense of mastery, Feeling toward change \\
\hline & & Other values/ Traits and Beliefs & $\begin{array}{l}\text { Trait anxiety, Universal values, Time orientation, Materialism, } \\
\text { Environmentalism, Concern for physical appearance, Religiosity, Cultural } \\
\text { strength, Level of superstition, Female role, Familism }\end{array}$ \\
\hline \multirow[t]{3}{*}{$\begin{array}{l}\text { 3.4.2. Life in } \\
\text { Transition }\end{array}$} & $\begin{array}{l}\text { 3.4.2.1. Transition Adaptation } \\
\text { Adjusters }\end{array}$ & Information Acquisition & $\begin{array}{l}\text { Information seeking activity, Information sources and usefulness, Antenatal } \\
\text { classes }\end{array}$ \\
\hline & & $\begin{array}{l}\text { Information Processing and } \\
\text { Utilisation }\end{array}$ & $\begin{array}{l}\text { Information processing self efficacy, Perceived information benefits and } \\
\text { outcomes, Difficulties and barriers processing information, Change as a } \\
\text { result of information, Arranging pregnancy care }\end{array}$ \\
\hline & & $\begin{array}{l}\text { Other Pregnancy Attitudes and } \\
\text { Drivers }\end{array}$ & $\begin{array}{l}\text { Affinity for technology in pregnancy, Pregnancy Health Locus of Control, } \\
\text { Pregnancy health effort and motivation, Achievement motivation, Approach- }\end{array}$ \\
\hline
\end{tabular}


3.4.2.2 a. The Transitional Self Nutrition (Physical)

\section{Exercise and Rest}

Substance Use

Risk and Risk Reduction

3.4.2.2 b. The Transitional Self Support (Psycho-social)

\section{Coping}

Experiencing the Pregnant Body
Nutrition attitudes, Nutrition practices, Reducing nutritional risk. Barriers to good nutrition

Attitudes and knowledge about exercise, Exercise practices, Barriers and interference to exercise, Sleep and rest patterns

Tobacco use, Alcohol intake, Medication use, Illicit drug use

Dental hygiene, Pre-natal screening, Environmental safety

Sources support, Satisfaction with living environment

Anxiety, Emotions and reactions, Relaxation and stress reduction Pregnant body image, Appearance interest, Celebrity attraction

Perception of wellness

Maternal/fetal attachment

Leaving childless days behind, Looking forward to the future 


\subsection{FRAMEWORK DIMENSIONS, CONSTRUCTS AND VARIABLES EXPLAINED}

The Influential Lifestyle Factors dimension contains three sub-domains of constructs and attributes present at the outset of pregnancy: socio-demographic factors, pre-existing influences (values and personality traits) and circumstances surrounding the pregnancy. The Life in Transition dimension encompasses constructs and attributes that represent the manifest active part of the transition journey: 'Transition Adaptation Adjusters' and 'The Transitional Self'. The Adaptation dimension contains constructs reflecting 'Transition Success'.

This following section discusses each dimension of the framework and its constituent elements in a consecutive fashion. For easy reference, constructs and attributes that represent groups of variables assigned to them are bolded and italicized and variables are in double quotation marks. If during the course of discussion about a variable any items or scales used later for measurement are also discussed, these are denoted by single quotation marks.

\subsubsection{INFLUENCING LIFESTYLE FACTORS DIMENSION}

Constructs and attribute sets assigned to the Influencing lifestyle factors dimension exist at the outset of pregnancy. Socio-demographic factors contribute to the diversity in the pregnancy market found particularly in ethnic make up and increasing age range for a first pregnancy. Additional socio-demographic factors were identified that can influence aspects of life in transition. The Pregnancy circumstance or events surrounding the pregnancy may also strongly influence attitudes and motivations which are manifested in behaviour based on choice and/or circumstances. Each sub-domain is now discussed in detail.

This dimension includes values and personality traits that are believed to have an impact on lifestyle choices that might be made during pregnancy transition. Measurement of relevant traits and values is important, as they evaluate perceived and self rated interest and commitment to health, along with ability to control and manage personal life affairs in general. As traits and values would most likely exist at the pregnancy commencement and remain relatively stable, they may have a predictive value for how a person's pregnancy health behaviour will be enacted. This sub-domain of Influential lifestyle factors contains two constructs and a set of eleven other values and traits presented as an attribute set, rather than a construct. 


\subsubsection{Socio-demographic sub-domain}

This sub-domain is comprised of the socio-demographic attribute set made up of several socio-demographic makers that related to a person's life situation.

\section{Socio-demographic Factors attribute set}

This section of the framework contains socio-demographic factors, operationalised as variables that are important for profiling and also because of their known association with certain risks and outcomes of pregnancy. "Age", particularly at the extremes of the fertile years, can increase types of physical and social risk (Dobrzykowski et al., 2003; Myers et al., 2006; Amy \& Loeber, 2007; Friese et al., 2008). "Ethnicity", is associated with certain health practices and health status (Lu \& Halfon, 2003). "Household income", "Employment" and "Education" have an association with health consumerism and self-efficacy as they have all been shown to be associated with health lifestyle, health outcomes and access to resources (Chin et al., 2000; Lindsay, 2004; Joseph et al., 2007).

"Living situation" is made up of factors related to the type of situation in their home that influences living arrangements and processes. For instance, the 'Length of time in New Zealand' and the 'Language spoken at home' can be an indication of the level of acculturation, known to influence pregnancy beliefs and practices. Other factors regarded as influential to a pregnancy health context are the 'Contact with the baby's father, and the 'Household structure'. These factors are of interest, as they may provide an indication of intimate social support structure, parenting responsibilities (for example the partner's children) and access to resources (Gunter \& Furnham, 1992). They may also act as barriers or facilitators to health.

"Domicile" refers to the type of region a woman lives in can produce intraregional similarities and between region differences in health resources and lifestyle due to geographical boundaries and cultural similarities.

"Media use" is defined as the usage frequency of different media used by the women. Media use preferences and the frequency women access selected media influences exposure to difference types of information and demonstrates preference for how it is presented and acquired. It is included here rather than in the pregnancy information section as it pertains to usual patterns of behaviour, not just pregnancy access to information. Media use has been found to be an important facet of lifestyle (Vyncke, 2002). 


\subsubsection{Pregnancy Circumstance sub-domain}

The sub-domain of pregnancy circumstance is concerned with factors pertinent to how the pregnancy has occurred. It is made up of two constructs, Feelings about the Pregnancy and Pregnancy Dates and an attribute set, 'Other Experiential Factors'.

\section{Feelings about the Pregnancy}

"Pregnancy intendedness'" the measure of intention to become pregnant, can act as an influence over how one cares for their own health and watches over the baby's development. It also has an association with maternal-fetal attachment (Altfeld, Handler, Burton, \& Berman, 1997; Pulley, Klerman, Tang, \& Baker, 2002). For the purposes of this study, a division has been made between intended (at the time or a time before), mistimed (unplanned but not unwanted) and unwanted (most often unplanned as well). This is proposed to be a truer reflection of how women feel about the origins of pregnancy (Altfeld et al., 1997; Pulley et al., 2002; Speizer et al., 2004) as opposed to more traditional ways of intention segmentation: planned or unplanned, in which mistimed would be measured as unwanted. Some authors also believe that mistimed pregnancies have more in common with wanted planned pregnancies than with unwanted pregnancies (Pulley et al., 2002).

"Original intentions for the pregnancy" is a measure of contemplation to not enter into motherhood at the current time and includes options of termination and adoption contemplation. "Evaluation of pregnancy worth"" is how one appraises the pregnancy at this point in time and a "Rating as a life event" 'relates to the appraisal of the value of the pregnancy in their life. This will allow comparison back to pregnancy intendedness and resolve from earliest feelings. Pregnancy meaning has been found to have an influence over health lifestyle (Altfeld et al., 1997; Speizer et al., 2004; Pulley et al., 2002; D'Angelo et al., 2004). Therefore the 'Feelings about the pregnancy' variables are each potentially important influencers over how a woman approaches decisions about her pregnancy health lifestyle.

\section{Pregnancy Dates}

"Weeks now" is the respondent's current weeks of gestation. This variable indicates how far into transition a respondent has travelled and is also included because of differences in both physical and psycho-social status and adaptation 
that occur across the three trimesters (Tulman \& Fawcett, 2003). Clarification of these weeks of gestation is essential when making statements related to findings and comparisons within and between any clusters that may be found. "First awareness" the weeks at which respondents were first aware of their pregnancy' is included as a potential differentiator and one that might have linkages to other variables, such as intendedness. Knowledge of when the target audiences of social marketing messages are usually first aware of pregnancy is important for timing of messages and channel selection.

\section{Other Experiential Factors attribute set}

This set of attributes contains a pool of additional factors arising from the pregnancy circumstance that will be influential to the pregnancy transitional lifestyle. The "Level of risk" assigned to the pregnancy is a significant variable in a woman's perception (or reality) of how the pregnancy might threaten or reduce health for both herself and her baby (Gupton et al., 2001). Those who have a high level of physical risk will be under the increased surveillance of the health care system and a high social risk may flag potential problems in health maintenance and/or psycho-social adjustment (Gupton et al., 2001. "Knowing sex of baby", is the respondent's desire or interest to know the sex of the baby, and may be associated with distinct attitudes and behaviours (such as superstition, technology and approach-avoidance) and potentially attachment behaviours. "Previously pregnant" defined as 'whether one has ever been pregnant before the current pregnancy is included, as a previous pregnancy loss may influence pre-existing pregnancy knowledge or increase anxiety during the current pregnancy (although it is not determined in this study), if the previous pregnancy was an induced or spontaneous termination, which may result in different residual states. "Fertility assistance required", whether assistance by a health professional to facilitate conception was required, includes a range of intervention from simple advice to more major interventions, such as in-vitro fertilization. Difficulty conceiving naturally may potentially increase anxiety and may also lead to a higher level of risk and concern a woman has for her health during the pregnancy because of a 'precious' pregnancy (Balen, Naaktgeboren \& Trimbos-Kemper, 1996; Bhalla, Sarala \& Dhaliwal, 1992).

\subsubsection{Pre-existing influences sub-domain}

The Pre-existing influences sub-domain is made up of values, existing attitudes and personality traits that give rise to patterns of behaviour that will in some way 
continue to shape and influence the course of the pregnancy. It contains two constructs and one attribute set.

\section{Pre-existing Health}

Health values are defined firstly by "Health as a value" which is the importance placed on health, level of involvement and pursuit of health, signaling the level of involvement and value of health to a person (Dutta-Bergman, 2003). Higher levels indicate that care is taken with health and pursuit of good health.

"Health consumerism" is the extent to which an individual partakes in activities around health consumption that involve seeking information, participation in health matters and the level to which they will control relationships with a health provider (Park \& Mittal, 1985; MacInnis et al., 1991; Dutta-Bergman, 2003). It may be indicative of how involved, assertive and active a pregnant woman will be in the pursuit of the care they perceive to be needed for themselves or their baby. A high level of both "Health as a value" and "Health consumerism" has been positively linked to health promotion and maintenance attitudes and activities in previous studies (Dutta-Bergman, 2003).

"Pre-pregnancy health level" reflects the measure of one's personal health in areas such as nutrition, exercise and psycho-social health. This variable was informed by Cassidy (2000) at to what makes up health maintenance activity. A pre-pregnant level of health provides an anchor point from which to compare how a woman's health has moved or changed in pregnancy. Those with a low score for pre-pregnancy health may be challenged to obtain a high level of health in pregnancy compared to a woman who had been actively maintaining a high level of health pre-pregnancy.

\section{Mastery and Achievement}

"Sense of Mastery" is a person's perceived ability to control personal situations and direction in life'(Pearlin \& Schooler, 1978; Turner \& Donald, 1999; Ellison et al., 2001; Schieman et al., 2003; Pearlin et al., 2007). It is associated with a capacity to protect health and well being and a factor that would influence the effectiveness and belief a woman has in her ability to achieve desired goals and outcomes. These values are likely to be stable in a woman's existing selfperception and will be transferable in some way to the new situation of pregnancy 
As transition involves a period of significant change, "Feeling toward change" is defined as the like or dislike a person has toward change in their life was included as it is also likely to influence the way the pregnancy is embraced.

\section{Other values, traits and beliefs attribute set}

"Trait anxiety" is defined as a personality trait that determines a person's predisposition to anxiety which can affect health lifestyle by increasing one's tendency to worry (Spielberger, 1975). A high trait anxiety person is more prone to worry and is thought to have a greater predisposition to develop anxiety type illnesses (Spielberger, 1975). A predisposition to anxiety can arguably lead to increased state anxiety in novel experiences, which have an element of the unknown and are presented as potentially threatening (Spielberger, 1975). As the study focuses on transition to first birth as a novel experience physically and emotionally, it is feasible that those with high trait anxiety will develop higher pregnancy and related life state anxiety when compared to lower trait counterparts.

Values are defined as deeply held feelings about what is important in life (Goldsmith, Freiden, \& Henderson, 1995). "Universal values" are defined as high level values that guide behaviour (Kahle \& Kennedy, 1988). The Excitement, dimension in Kahle's List of Values (Kahle, 1984) has been linked to health maintenance (Kraft \& Goodell, 1993; Bearden, Netemeyer, \& Teel, 1989; Divine \& Lepisto, 2005; Dutta-Bergman, 2003). Those who value excitement highly, generally have a lower level of commitment to health in the longer term. "Time orientation", the extent to which one is prepared to substitute the future for immediate gains (Hendrix, 1984; Hornik, 1990) has been shown to indicate that those who plan ahead are more likely to maintain a healthy lifestyle and are more likely to forsake activities such as tobacco smoking (Hornik, 1990).

"Materialism", the importance of and the level to which people pursue possessions and money for personal happiness and progress, has been negatively linked to pro-environmental attitudes (Tilikidou \& Delistavrou, 2001; Schnaninger \& Lee, 2002), which in turn have been linked to positive health attitudes in consumers. Those high in materialism may also be more concerned with potential loss of earnings through disruption to work patterns that parenthood may bring. "Environmentalism", is defined as the extent to which a person is concerned about the caring for and preserving environmental resources. A high level of environmental consciousness has been positively associated with health 
consciousness consumer, as demonstrated by high levels of positive nutritional attitudes and practices (Dutta-Bergman, 2003) and may influence products that are purchased to pursue pregnancy health (Dutta-Bergman, 2003). "Concern for physical appearance", defined 'as a concern for one's physical appearance' (Netemeyer, Burton, \& Lichtenstein, 1995) may influence the use of maternity appearance products used to reduce a woman's real-to-desired self image gap. Those with a high level of concern for physical appearance may feel resistance to acceptance of the physical changes that pregnancy brings, particularly if they normally strive to maintain the stereotypical image of ideal female shape.

A high degree of "Religiosity" the extent to which a person believes religion influences their life, has been found to be linked to positive health practices and effort and mastery (Schieman, et al., 2003), and may be a factor in the degree to which a person holds health to be controlled by an External Health Locus of Control (Wallston et al., 1978). "Cultural strength" is defined as the degree to which a woman's affiliation and commitment to a particular culture informs and influences values, beliefs and practices about childbearing and direct preferences for the type of healthcare sought (Liamputtong et al., 2005; Ayaz \& Efe, 2008). The importance to which someone wishes and is able to live according to their culture can facilitate or frustrate the experience of pregnancy. Adherence to one's original culture may be a protective agent in pregnancy (McGlade et al., 2004). "Level of Superstition", the extent to which a person believes superstitious beliefs and practices influences their life (Rudski, 2003) will form part of the complex backdrop that makes up a woman's beliefs and fears about pregnancy. A relationship between 'cultural adherence strength' and the importance of adhering to superstitious practices has also been found (Liamputtong et al., 2005; Rudski, 2003).

Attitudes toward the "Female role'" defined as beliefs about the role and priorities of women in society' can guide a woman's attitudes and behaviour ( $\operatorname{Sin} \&$ Yau, 2004). It can be a possible influencer in how pregnancy is rated by a woman and how the pathway toward motherhood is negotiated, particularly from a psychosocial perspective. Likewise, "Familism"" the strength of family bonds and loyalty to the family (Luna et al., 1996), may influence the felt and expected obligations between family during pregnancy and the meaning and type of support sought. 


\subsubsection{LIFE IN TRANSITION DIMENSION}

The dimension Life in Transition contains the sub-domains of Transition Adaptation Adjusters, and the Transitional Self. This dimension further reflects the sequential passage through the transitional lifestyle and is the period when major adjustment is occurring and manifesting in behaviours that are pregnancy specific or related.

\subsubsection{Transition Adaptation Adjusters sub-domain}

The Transition Adaptation Adjusters sub-domain contains constructs and variables considered to be key drivers of transition; factors that propel a person along the transition course in a forward motion and thus lead to some form of change and adjustment. There are three major constructs. Information Acquisition and Information Processing and Utilisation are concerned with the way in which women gain knowledge and information related to pregnancy, and then process and make decisions, or pursue certain courses of action that will maximize their wellbeing. The third construct Major Attitudes and Drivers is comprised of variables that are formed in response to their situation and motivations, barriers and facilitators that impact on behaviour.

The Information constructs presume that in this context, information sought and used is underscored, mainly, by rational consumer decision making and consumer choice. In order to make changes, a consumer needs to acquire relevant information, process it effectively and then determine the range of alternatives that are possible. To do this, consumers typically evaluate alternatives and decide on a course of action. At the same time, evaluation of the benefits and drawbacks, (including barriers to change), and decision options (such as in the Precaution Adoption Process Model) will provide extra cues as to the course of action that is best for the individual. Seeking and using information is a key driver in adaptation to the pregnant health lifestyle.

As in complex decisions made in a consumer decision making framework, the ability to understand the range of options, determine and evaluate between them and then make a purchase decision is dependent on quality of information available and the motivation and ability one has to process it in a meaningful way. In a consumer decision making context, existing attitudes contribute significantly and can assist as cues where prior experience is minimal. New information and persuasion may also be helpful in decision making. Too much or 
confusing information may confound the ability to process coherently. Preexisting attitudes and those formed for a specific context can be changed as a result of information or persuasion. The combination of these factors, along with motivations, will guide behaviour.

The potential for consumers in this context to engage in rational or experiential decision making has been accommodated within the framework, by recognizing that consumers do not only determine a course of action through external information sources, but additionally utilize other ways of knowing. As discussed in the literature review, this includes for instance, 'listening to one's body' as a means of adaptation. In addition, to information related factors, several other pregnancy motivations and drivers will underpin adaptation.

\section{Information Acquisition}

Although there will be an existing level of knowledge at the beginning of pregnancy, "Information seeking activity", (McKenzie, 2001) the level of interest and activities engaged in to acquire pregnancy information and reduce an acknowledged knowledge gap commences in the early part of the pregnancy and continues throughout the duration of pregnancy, with different needs and focus at different stages of pregnancy progression. For instance, the need for information about labour and birth becomes more important in the third trimester. Information seeking activity will be underscored by a woman's belief in the importance of information and felt responsibility for gaining it (Deutsch et. al, 1988; Maher, 2000; McKenzie, 2001 Dutta-Bergman, 2003). Information acquisition is acknowledged through many 'Information sources' and 'Source usefulness'. These measures relate to is the processes of acquiring information, and the level of usefulness perceived by a woman that a particular source has as a pregnancy health informant and confidence in the value of the message type (Dunn et. al, 2003). "Antenatal classes" is defined as the transfer of knowledge regarding pregnancy issues by a teacher (usually a health professional) in the antenatal period. It has a more formal delivery and organization than spontaneous interaction. Whilst usually occurring in a group situation, it can also be held in a one-to-one setting. A woman may have different information needs, which she wishes these classes to meet, and for that reason will select from the various offerings available. 


\section{Information Processing and Utilisation}

Whilst a vast amount of information reaches women during pregnancy, it needs to be effectively processed in order for it to be useful as a Transition Adaptation Adjuster. Many factors, both internal and external, will impact on the woman's ability to undertake this. "Information processing self efficacy" is the confidence one has to receive and process information (Aldoory, 2001; Dunn et al., 2003; Wu and Chung, 2003). "Perceived information benefits and outcomes" make up the beliefs about advantages of having and using information in the pregnancy period. Likewise, "Difficulties and barriers processing information" are internal and external factors that detract from information processing and potentially interfere with usage effectiveness. "Change as a result of information" are the changes to pregnancy health attitudes and behaviour undergone as a result of information gained' The degree to which these are positive may be dependent on several factors (Aldoory, 2001; Levy, 1979).

"Arranging pregnancy care" is regarded as an outward expression of acknowledgement of the pregnancy. It is defined as the activities a woman engages in to assure pregnancy healthcare and a place to give birth are arranged. Some of decisions made, such as type of care and intended place of birth, may have been elaborated on during a pregnancy planning stage. However decisions for most women will be complex and elaborated on with some degree of care and referral to others for guidance. The process and degree of ease reported may also be a function of regional access to care, geography and the type of provision available in the region where a woman lives. The 'Intended place of birth' reflects the preference for birth as a home or hospital event. Arguably, this variable could also be assigned just as well as a risk reduction behaviour as it is a health promoting behavior here. For instance, regular visits to the doctor are regarded as a component of health maintenance (see for example, Pender 1987). However, given that all the women in this sample were receiving pregnancy care (having been accessed through the health system) this variable has been assigned to the Information Processing and Utilisation construct. 


\section{Other Pregnancy Attitudes and Drivers}

Variables contained within the Other Major Attitudes and Drivers construct serve as stimuli for how behaviour patterns develop in response to the demands of transition. "Affinity for technology in pregnancy" is the acceptance of and reliance a woman places on surveillance of the pregnancy through technology (Taylor, 2000). "Pregnancy Health Locus of Control", based on Multi-dimensional Health Locus of Control work (Wallston et al., 1978) and Fetal Health Locus of Control (Labs \& Wurtelle, 1986; Kruse et al., 1988), demonstrates the extent to which one feels the power over pregnancy health is internally or externally controlled. "Pregnancy health effort and motvation" is defined as the level of effort and focused activity engaged in to promote health in pregnancy. High levels of motivation and effort are known to be enablers in facilitation of goal driven action and direct behavioural outcomes and engagement in good health practices. "Achievement motivation"" is the drive a person feels and the intensity to which one strives to achieve goals (Allport, 1955; Cassidy, 2000; Sideridis, 2007). This is likely to affect the energy put into pursuing goals and feelings about achievement or non-achievement. For someone with a high degree of achievement motivation, goal failure, (whether through internal or external factors) could bring about a significant sense of disappointment. Likewise, "Approach/avoidance" motivation, defined as the extent to which a person will approach or avoid a potentially stressful or fearful situation (Elliot, 2006), would influence the desire to be involved in decision making and knowing if something untoward is occurring with the pregnancy or developing baby. "Enablers and barriers to change" are the factors which act as enablers or barriers in making health changes. "Reasons for health changes" are the reasons that cause a response to health change in a positive or negative direction; these may be welfare of self generated, response to others generated or welfare of baby generated.

\subsubsection{The Transitional Self sub-domain}

This is the attitudinal and behavioural expression and outworking of the preceding constructs and attributes already discussed. Attitudes that are specific to a particular behaviour (for example, nutrition and exercise) are shown as part of the Transitional Self, as compared to the preceding stage where more general pregnancy health attitudes were assigned.

The Transitional Self sub-domain is made up of both physical and psychosocial factors and for readability and clarity, there are discussed under two headings: 
physical and psychosocial. As this framework has a mainly health rather than an illness disease avoidance focus, informing health maintenance frameworks (for example, Pender) have been preferred for this study's conceptual foundation more so than disease-centric models. Pender's Health Promoting Lifestyle II (1987) contains six dimensions, across which health promoting behaviours are enacted; responsibility, physical activity, nutrition, spiritual growth, inter-personal relations and stress management are all incorporated within the conceptual framework of this study. However, a health promoting lifestyle framework needs to be adapted to the pregnancy situation, as it does not allow for the physiological and psychological changes inherent in the developing gravid state and period of transition. Therefore, pregnancy specific variables were identified and developed to complement other known factors within the overall lifestyle framework.

This sub-domain is made up of constructs related to physical and psycho-social components of the Pregnancy Health Transitional framework. Life in transition is made up of manifest behaviours and in this section included with them for clarity attitudes directly associated with that behaviour. The physical constructs and attributes include Nutrition, Exercise and Rest Substance Use and 'Risk Reduction'. Psychosocial constructs include Support, Coping and Experiencing the Pregnant Body. The inclusion of all these factors leads to a more holistic approach to lifestyle measurement than if only one or two of these constructs had been investigated. The assignment of variables has been undertaken for measurement purposes; however the author acknowledges that, arguably, many of these constructs in reality cannot be separated easily as strictly physical or psycho-social manifestations. For instance, violence in pregnancy holds severe known risks to both the physical and emotional safety of a woman. Likewise, exercise in pregnancy has both beneficial physical and stress reduction characteristics.

\subsubsection{The Transitional Self (Physical)}

\section{Nutrition}

The Nutrition construct contains a number of variables that reflect the range of aspects this construct encompasses. It measures attitudes and beliefs about the outcomes of good nutrition, as well as actual practices increasing the likelihood of optimal fetal nutrition. Factors that make up "Nutrition attitudes" are made up of attitudes that underscore nutrition and outcome beliefs; Positive attitudes increase the likelihood of positive practices (Chomitz et al., 1995; Fowles \& Feucht, 2004). The experience of eating pattern disturbance from nausea, 
vomiting and cravings, as well as attitudes toward weight gain, will have consequences for practices. "Nutrition practices" are defined as factors that incorporate nutritional effort afforded to eat well and actual intake against recommended pregnancy nutrition guidelines. "Reducing nutritional risk" involves the extent to which recommendations for foods to be avoided in pregnancy because of the risk of toxoplasmosis and listeriosis are adhered to and folic acid vitamin supplementation. Folic acid deficiency is associated with neural tube defects (Bondarianzadeh, 2007; Delgado, 2008; Ross et al., 2006) and considered an important vitamin in the early part of pregnancy. Arguably also, this variable could be within the Risk and Risk Reduction construct, but has been accommodated here because of its fit with the Nutrition construct), "Barriers to good nutrition" contains intrinsic and extrinsic influences, such as feeling unwell, time, poor cooking facilities (SPARC, 2003; Fowles \& Feucht, 2004) can act as constraints, potentially reducing the ability to undertake good food practices.

\section{Exercise and Rest}

Exercise is known to be beneficial to a healthy lifestyle and moderate exercise is recommended in pregnancy as a means of maintaining health (Chomitz et al., 1995; Clarke \& Gross, 2004; Symons et al., 2004; Ministry of Health, 2004). Exercise I variables include "Attitudes and knowledge about exercise". This variable is comprised of perceived benefits but counter balanced by factors such as dangers they feel the baby might encounter through maternal exercise. In order to feel confident about planning an exercise regime, information about exercise must also be accessible and sufficient. "Exercise practices" can be determined by type, frequency and whether there is an exercise plan that formalizes and organizes a woman's approach to exercise "Barriers and interference to exercise" are factors that reduce one's ability to exercise and can be internal (such as fatigue) or external, (such as lack of resources or weather). "Sleep and rest patterns", the patterns and amount of sleep and rest a woman adopts during pregnancy are generally increased from non-pregnant patterns. Patterns can be disrupted due to physical or structural discomforts and/or psycho-social concerns, such as anxiety.

\section{Substance use}

Substance use incorporating "Tobacco use" "Alcohol intake", "Medication use" and "Illicit drug use" refer to attitudes and usage and are of particular interest, due to the potential teratogenic and damaging effects these agents can have on fetal growth and development (Chomitz et al., 1995). Avoidance of tobacco, illegal 
drugs and a moderate intake of alcohol are behaviours recommended within a health-promoting lifestyle. In pregnancy in New Zealand, a health promoting lifestyle includes abstinence from alcohol for the pregnancy period and care with medication, including Over-The-Counter, natural or homeopathic and prescription medication (Chomitz et al., 1995; Butler et. al, 2004; Haslam \& Lawrence, 2004; Ministry of Health, 2006; Stewart \& Streiner, 1994).

\section{Risk and Risk Reduction}

"Dental hygiene", the extent to which one cares for periodontal and dental health during the period of pregnancy is an important part of health maintenance in pregnancy and included due to the potential for gum inflammation and periodontal disease that can arise during and from this period (Lydon-Rochelle et al., 2004).

"Prenatal screening" is defined as medical and/or technological surveillance and tests to screen for any situation that may potentially be harmful to a mother or baby, so that monitoring or intervention can occur. Although pre-natal screening is primarily a risk reduction and surveillance activity, the increased sophistication of technology and sharing of information between a provider and patient means that scanning performs other functions in the pregnancy health lifestyle for a woman (Taylor, 2000). It is also important to note that the benefits of pre-natal ultra sound scanning are controversial and some providers and pregnant women view ultra-sound scanning of the fetus as an unnecessary and/or potentially dangerous practice (Maršál, 2010).

"Environmental safety", the care taken to reduce threat or injury brought about by the environment is also an important component of health promoting lifestyle frameworks and behaviours in pregnancy, as a means of ensuring safe passage (Rubin, 1967; Heaman-Gupton \& Gregory, 2004). For example, 'Wearing a safety belt' in a car can be seen as a specific risk reduction activity. Environmental risk may also be associated with violence. Threats to safety or injury (physical, emotional or both) can lead to acute situations such as placental abruption. Violence can also be associated with poorer outcomes, such as low birth weight due to associated health lifestyle behaviour (for example, delaying care) (Chomitz, et al., 1995). The 'Experience of violence' measured within the environmental safety variable is a woman's actual experience of perceive threats to personal safety through violence. 


\subsubsection{The Transitional Self (Psycho-social)}

\section{Support}

A good level of support is linked to positive outcomes and may mediate other adverse behaviours (Feldman et al., 2003; Dejin-Karlson \& Ostergren, 2003; Tulman \& Fawcett, 2003). Quality of support will influence the type of emotions and enjoyment of life felt during the pregnancy. Support can consist of a range of factors, including emotional support, activities concerned with preparation for birth and the arrival of the baby, practical support (for example, housework) and financial assistance. "Sources support" is defined as 'the level of support desired and the perceived level received from those significant to a woman's life during pregnancy'. "Satisfaction with living environment" is defined as 'the level of satisfaction a woman has with her physical surroundings and accommodation and the type of social environment and networks with which she lives'. It is of interest as accommodation difficulties related to comfort and resource access may affect the ability to maintain healthy lifestyle and feel positive about the future (Chomitz et al., 2003).

\section{Coping}

Because of change a woman encounters during this transitional lifestyle, coping is an important function of psycho-social health. Coping resources are dependent on several factors; such as pregnancy risk level, a history of avoidance, depression and low self esteem (Walker, Cooney, \& Riggs, 1999; Huizink et al., 2002). "Anxiety", as used within the coping construct, refers to the level of anxiety directly related to the pregnancy itself (for example, apprehension about the birth) or due to a present stressful life situation, derived from broader concerns generated as a result of present circumstance'. Anxiety can cause difficulty with coping, transition adjustment and may lead to poorer outcomes, such as a baby 'small for gestational age' (Salmela et al., 2001; Dejin-Karlson \& Ostergren, 2003) as high levels of circulating cortisol may pose a risk to the developing fetus (Cassidy, 2000; Walker et al., 1999; Stark, 2001).

"Emotions and reactions" is defined as the feelings evoked and responses made to react to a particular situation. Pregnancy is a time where women report feeling changes to their usual responses to and being more emotionally labile (Huizink et al., 2002). This may also alter their 'problem solving' style. As a strategy to cope in pregnancy, decisions that are rationally or emotionally based may be used and emotion focused coping or problem focused coping may be employed (Huizink et al., 2002). 
"Relaxation and stress reduction", the activities a woman engages in to relax or reduce stress is fundamental to stress reduction and enjoyment and a healthy lifestyle in pregnancy. A focus on stress reduction and relaxation by women is a way of assisting in their adjustment to the changes and demands brought about by pregnancy. Several relaxation activities may be engaged in.

\section{Experiencing the Pregnant Body}

"Pregnant body image", is defined as the extent to which one enjoys their pregnant state and feels themselves attractive (Devine et al., 2000). If one thinks they are attractive, psychosocial wellbeing is likely to be enhanced (Earle, 2003; Strang and Sullivan, 1985; Johnson et al., 2004). "Appearance interest", the level of interest shown in increasing one's perceived attractiveness in pregnancy' is defined for this study as a positive response to a positive body image, whereby feeling good about oneself increases the interest taken to appear attractive. An intense degree in appearance interest could be an indication of extreme vanity, or another factor causing distortion. This could expose a woman to stress brought about through attempts to gain control over her gestating body. "Celebrity attraction", the extent to which one relies on celebrities for image cues, serves as a potential source of vicarious learning and behaviour adoption, but may interfere with the setting of realistic goals, and satisfaction with self (Longhurst, 2004).

\subsubsection{ADAPTATION DIMENSION}

The Adaptation dimension is comprised of the sub-domain 'Transition Success'; those factors that epitomise what is considered a healthy response and adaptation, particularly as informed by Schumacher and Meleis (1994), to the Transitional Health Lifestyle of Pregnancy.

\subsubsection{Transition Success sub-domain}

The dimension of Adaptation incorporates one sub-domain: Transition success. The conceptual basis for this sub-domain is informed by Schumacher and Meleis, (1994), as being indicative of positive adaptation in a life transition and modified for a pregnancy transition situation. While the measures of transition success are indicative only (being taken during the time of pregnancy), they are included to allow for at least a provisional assessment of how well the woman was adapting to the pregnancy transitional lifestyle. They hold potential to complement measures in future research taken after the actual birth of the child (for instance, birth weight, clinical assessment at birth and 'thriving' in the post-partum), in a 
longitudinal study of maternal and child development. The variables within this sub-domain are discussed below.

\section{Wellbeing}

A "Perception of wellbeing" is generally accepted as a sign of successful transition as it occurs when feelings of distress or disorganization are replaced with holistic feelings of wellness (Wallace et al., 2009). Schumacher and Meleis (1994) believe that in pregnancy, this construct extends to both physical and psychological perceptions. Wellbeing is therefore defined as a sense of holistic wellness.

\section{Relationship with the Baby}

During pregnancy, a growing positive relationship with the baby is important for emotional wellbeing and to ensure the mother is motivated to provide an optimal intrauterine environment for the baby (Müller, 1994). "Maternal-fetal attachment" is indicative of 'the growing attachment to and positive relationship with the baby' (Müller, 1994), whereby positive emotional attachment to the fetus is indicative of anticipation of a baby and motherhood. A low score may be indicative of problems in the post-natal period (Müller, 1994; Boyce \& Corkindale, 1997).

\section{Orientation to the Future}

Looking positively to the future can be regarded as a positive transition signal and forward progress (Rubin, 1987; Mercer, 2004; Barba, \& Selder, 1995; Schumacher \& Meleis, 1994; Devine et al., 2000). The variables in this construct are "Looking forward the future'", which is defined as 'positive anticipation of a new life stage' and "Leaving childless days behind", which is contentment that one is leaving a life stage where there was no responsibility for children'

\subsection{CONCEPTUAL FRAMEWORK SUMMARY}

The main stages of the conceptual framework are those that are pre-existing and therefore are influential at the beginning of and during the pregnancy course. These include pre-existing values and traits, demographics and the circumstances of the pregnancy. Women adjust to pregnancy during transition and make decisions regarding their pregnancy based on a range of information, acquired through various sources. Information acquired must be processed effectively before it is used to inform and influence decisions, particularly those that may result in modification or changes to health lifestyle as a result. The extent to which information underpins attitude formation and action depends on several factors, including intrinsic motivations and perceived enablers and constraints. 
Attitudes include Health Locus of Control (dictating the extent to which a woman believes her pregnancy health is within her control or another's) and attitudes toward certain behaviours (such as exercise and tobacco use). The pregnancy lifestyle is enacted through various behaviours, many of which, but not all, are common to other health promoting frameworks. These are divided between physical and psycho-social dimensions. Lastly, transition success can be measured using factors which are known to demonstrate a positive adaptation and direction toward readiness for entry into a new life stage.

As a summary view, the conceptual framework for the study is presented diaphragmatically in Figure 3.2. Figure 3.2 provides a similar summary to Table 3.1 , but is represented in a graphical format for quick reference when reading subsequent chapters. 
Figure 3.2 Conceptual Framework: The Transitional Pregnancy Health

\section{Lifestyle by Sub-domain, Constructs and Attributes and Variables}

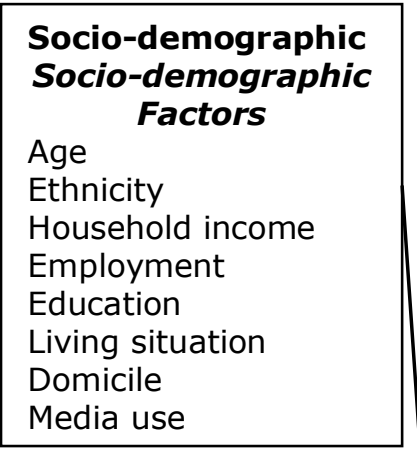

\begin{tabular}{|l|}
\multicolumn{1}{c|}{ Pregnancy } \\
Circumstance \\
Feelings about the \\
Pregnancy \\
Pregnancy \\
intendedness \\
Original intentions for \\
the pregnancy \\
Evaluation of \\
pregnancy worth \\
Rating as a life event \\
Pregnancy Dates \\
Weeks now \\
First awareness \\
Other Experiential \\
Factors \\
Level of risk \\
Knowing sex of baby \\
Previously pregnant \\
Fertility assistance \\
required
\end{tabular}

\begin{tabular}{|l|}
\multicolumn{1}{|c|}{ Pre-existing } \\
Influences \\
Pre-existing Health \\
Health as a value \\
Health consumerism \\
Self-reported health \\
Mastery and \\
Achievement \\
Sense of mastery \\
Feeling toward change \\
Other Values/ Traits \\
and Beliefs \\
Trait anxiety \\
Universal values \\
Time orientation \\
Materialism \\
Environmentalism \\
Concern for physical \\
appearance \\
Religiosity \\
Cultural strength \\
Level of superstition \\
Female role \\
Familism \\
\hline
\end{tabular}

\section{Transition Adaptation Adjusters}

\section{Information}

Acquisition

Information seeking activity

Information sources and usefulness

Antenatal classes

Information

Processing and Utilisation

Information processing self-efficacy

Perceived information benefits and outcomes Difficulties and barriers processing

Changes as a result of information

Arranging pregnancy care

\section{Other Pregnancy}

Attitudes and Drivers

Affinity for technology

Pregnancy Health Locus of Control

Pregnancy health effort and motivation

Achievement motivation

Approach-avoidance

Enablers and barriers to change

Reasons for health

changes
Transitional Self (physical)

\section{Nutrition}

Nutrition attitudes

Nutrition practices

Reducing nutritional risk

Barriers to good nutrition

Exercise and Rest

Attitudes \& knowledge about

exercise

Exercise practices

Barriers and interference to exercise

Sleep and rest patterns

Substance use

Tobacco use

Alcohol intake

Medication use

Illicit drug use

Risk and Risk Reduction

Dental hygiene

Prenatal screening

Environmental safety

Transitional Self

(Psychosocial)

\section{Support}

Sources support

Satisfaction with living

environment

\section{Coping}

Anxiety"

Emotional and reactions

Relaxation and stress reduction

Experiencing the Pregnant

Body

Pregnant body image

Appearance interest

Celebrity attraction

\begin{tabular}{|l|}
\multicolumn{1}{|c|}{ Transition Success } \\
Wellbeing \\
Perception of wellness \\
Relationship with the \\
Baby' \\
Maternal/fetal attachment \\
Orientation to the Future \\
Leaving childless days behind \\
Looking forward to new life \\
stage
\end{tabular}




\subsection{COMMENT ON ADDITIONAL FACTORS CONSIDERED IN THE DESIGN OF THE FRAMEWORK}

Although most of these phenomena of interest were identified from the health and marketing literature, many variables for the Pregnancy Health Framework had not been tested specifically in a pregnancy context. Therefore, the framework was developed in part to explore a number of factors that could be influential over pregnancy health lifestyle. These included assessing the appropriateness and fit of such factors for a Pregnancy Health Lifestyle tool for future research.

Development of a pregnancy health lifestyle typology, while perhaps appearing straightforward, poses some complex conceptual considerations. The purpose of this study was not to measure a respondent's level of health per se, or to illuminate only those respondents who are engaging in a health promoting lifestyle. Rather it sought to cluster attitudes and behaviours into meaningful aggregations, whether they be health promoting, health detracting or a combination of both. It needs to be made clear that there was no objective clinical evaluation of each woman's health, or application of measures that would enable scientific comparison in terms of, for example fetal growth, blood pressure recording or physical fitness levels. However, the clustering methodology employed is well suited to a holistic approach, with a capacity to systematically handle a large variable set in identifying heath lifestyle segments.

Many phenomena of interest overlap across both the marketing and health disciplines. For instance, 'body image' and its influences over an individual are applicable to both clinical and marketing lifestyle studies. Extreme involvement and vulnerability toward body image and self esteem can lead an individual to practice poor nutrition behaviours that lead to an eating disorder or, for a marketing consumer striving to obtain their ideal body image, to engage in heavy consumption of body enhancing products in an effort to bridge the perceived gap between the real and ideal. Therefore, for this study, purchase of goods to support health behaviour (for example, dietary supplements and education) are seen just as rightly as health behaviours as the amount of fat intake in a diet. Furthermore, assignment of variables to one location within the framework can also be problematic. Some health-related behaviours (for instance, alcohol intake) may also be social behaviours and therefore some of the variables selected in this study could very rightly have been allocated to more than one 
position in the framework. Ultra-sound scanning could be included in 'affinity for technology' as well as 'screening for risk reduction'. It is not an insurmountable difficulty however, as the more pressing concern was to ensure that variables were at least included in the framework. It is hoped that, with the wide ranging and cross disciplinary literature review undertaken, all critical constructs and variables were included.

This study relies heavily on respondent's perception of their own health lifestyle. Although, there can be some statements made about pattern or idiosyncrasy assigned to different groups, care needs to be exercised in not hastily characterizing groups as 'good' or 'bad', except by what can be referred back to evidence in the literature from other sources or studies. A further consideration is that what is health promoting behaviour in pregnancy, in some instances, depends on a person's own philosophy and point of view. For example, having a scan could be seen by some women as an essential risk reduction activity, where for others, the scan could be seen as an unnecessary intervention with potentially unknown negative consequences in the long term. Overall, it is believed that the conceptual framework has provided significant insights and an important contribution towards an understanding of pregnancy as a transitional health lifestyle.

\subsection{USE OF THE CONCEPTUAL FRAMEWORK TO GUIDE ANALYSIS}

The conceptual framework guides subsequent analysis by assuming order through the transitional life stage of pregnancy. The framework assumes that pre-existing circumstances may well influence how the pregnancy lifestyle is enacted. The gathering of data related to pre-existing circumstances allows for comparisons to be made during data analysis. Likewise, information is posited as a key adjuster to the new demands of lifestyle adjustment. Whilst pre-existing circumstances are present at the threshold of a woman's pregnancy health lifestyle, information related to pregnancy is mostly obtained substantially during planning for pregnancy and then more directly during the pregnancy (McKenzie, 2002; Root \& Browner, 2004). It is acknowledged that women will have some pre-existing attitudes toward pregnancy, formed at earlier stages; however, this study does not specifically focus on distinct separation of the two information source stages. Information processing and application is assumed to influence the enactment of health behaviours. Again, much of the measurement extracts information that enables it to be compared with what is exemplary of a health promoting 
pregnancy lifestyle, as determined through the literature review and one that will ensure maximum positive outcomes for mother and baby. Transition success is the final sub domain specified. Its aim is to provide a degree of closure to the enquiry and provide some estimation of which path through the pregnancy health lifestyle is most associated with the most positive transition success scores.

In summary, the study's framework is an attempt to respond to one of the persistent criticisms of lifestyle that is atheoretical (Wells, 1975; Anderson \& Golden, 1984). The framework devised is a first attempt to look for holistic collectivities of progression paths. The precise predictive capability of this framework is not tested by hypothesis in this exploratory study

\subsection{CHAPTER 3 SUMMARY}

This Chapter has presented the conceptual framework, which includes the stages through which pregnancy health lifestyle transition occurs. An introduction to the framework was given, followed by presentation and discussion of the framework. Research questions were then framed that explore the conceptual framework. Sub-domain variables have been defined with referral to other author's work that informed the construction of variable definitions.

The following Chapter 4 explains the study design and how the conceptual framework was operationalised, the collection of data and analysis of results. 


\section{CHAPTER 4 STUDY DESIGN}

\subsection{INTRODUCTION}

In the previous chapter the conceptual framework and research questions were developed and explained. The purpose of this chapter is to detail cross-section survey design to address the range of research questions developed in Chapter 1.

\subsection{STUDY APPROACH}

Integration and synthesis of the existing literature led to several emergent dimensions and variables that have been brought together in the conceptual framework for the Transitional Health Lifestyle of Pregnancy described in Chapter 3. The full impact of variables, their interaction and the extent of their influence could not be determined prior to data collection. Therefore, this study is ex-post factor and exploratory rather than confirmatory or experimental.

In order to obtain the data required to measure variables that would efficiently explore the research questions, the design selected for this study was a crosssectional survey. A study using a cross-section design involves the collection of data on more than one case at a single point in time. It is undertaken in order to collect a body of quantifiable data in connection with two or more variables, which are then examined for patterns of association (Bryman \& Bell, 2003).

Survey methodology is widely adopted in social science and business related research (Malhotra, 2007; Cavana, Delahaye, \& Sekaran, 2001). It allows for a large amount of data to be collected for a large representative sample of people. A survey is defined as 'a research strategy in which quantitative information is systematically collected from a relatively large sample taken from a population. A questionnaire is an efficient and economic way to capture data in a survey. Questionnaires are a very common way to carry out a survey (Malhotra, 2007; Cavana, Delahaye, \& Sekaran, 2001). The questionnaire is developed whereby the researcher knows what information is needed to be gathered about each variable. The measurement instruments were developed as part of the survey. Survey design is a very acceptable way of obtaining psychographic data and the data sought is most commonly collected by questionnaire (Wells, 1975). 


\subsection{OVERVIEW OF THE DESIGN}

A cross sectional design was selected for this study. Although a longitudinal study would have been beneficial for its offer of unique insights and process into change and continuity of phenomena over time (McGrath, 2003; Wadsworth et al., 2003) and therefore the end outcome of a life course transition, it was rejected. This was due to the desired goal of obtaining respondent perceptions as they were directly experiencing pregnancy. Although a longitudinal design would have meant that birth outcomes would have been known, and thus more conclusive evidence of the outcome of behaviours, there would have been timing and resource constraint issues. Furthermore, the experience of labour and birth could have coloured pregnancy impressions. In addition, response was sought as pregnancy was being experienced and not as a reflected historical event. The early first few weeks postnatal, particularly after the birth of a first baby, are known to be busy and the collection of a long questionnaire would have been difficult. If it was left too long, however, the finer detail of pregnancy heath may have been lost through memory decay.

\subsection{ETHICS AND ACCESS}

Ministry of Health ethics approval was required due to respondents being 'patients' in the health system, as primary access to them was through client bookings with health providers. In addition, in order to obtain as representative a sample as possible, both in terms of demographics and risk level, it required a somewhat complicated approach to many different healthcare providers.

Ethics approval was obtained through the New Zealand Multi-region Ministry of Health Ethics Committee, the forum for which New Zealand health research is considered. After initial consent, the process remained somewhat iterative, as each District Health Board approval gained required the approval of the committee chairperson.

The New Zealand Multi-region Ministry of Health ethics submission form was accompanied by information sheets and consent forms. This information to patients gave assurance that the researcher was not employed by a hospital, midwife or doctor and that participation would in no way affect their provider care. Reassurance was also given that the research was academic and not being undertaken for commercial gain. The researcher responded to committee 
questions and feedback by personally attending a committee meeting where the application was being considered.

A multi-site approval was sought due to the need to recruit widely and outside of Wellington to obtain sufficient distribution of questionnaires (based on estimations of a $20 \%$ response rate and minimum numbers required). Additionally, this would also increase geographical diversity (by size of town) and provide access to many more health providers. Confidentiality and treatment of raw data were arranged to meet the requirements within the ethics approval.

Ethics approval received from the Ministry of Health is acceptable to the Victoria University of Wellington (VUW) Human Ethics Committee. A full copy of the application was sent to the chairperson of the VUW ethics committee. Ethics consent was updated as per requirements of the Multi-region Ministry of Health Ethics Committee every twelve months.

Each approach to District Health Boards (DHBs) (for access to both primary and high risk clinics) and Primary Health Organisations (PHOs) required application and consideration through their individual research centres. Pursuit of DHB access was essential in order to access high risk women in secondary and tertiary care, achieve representation from the range of care type and reach the increasing number of women returning to hospitals for primary care.

Once an access agreement had been agreed and signed off, the appropriate documentation was forwarded to the chairperson of The Multi-region Ethics Committee for approval and alteration to the existing ethics cover. Where access was denied into a DHB, the reasons given were an existing heavy research load in the relevant departments at the time, or that staff were considered too busy. Access to these organisations was a long and sometimes complex process.

\subsection{CONSULTATION WITH MĀORI}

A requirement of the New Zealand Multi-region Ministry of Health Ethics Committee is that the intended research is consistent with the Treaty of Waitangi. Recognition of the concepts of Partnership, Participation and Protection, the three main governing principles of the Treaty of Waitangi, were acknowledged as an integral part of the development and execution of the study. A staff member from the School of Māori Business, Victoria University of Wellington, was 
consulted regarding wording and cultural appropriateness of the questionnaire, along with the cover image used and discussion regarding the overall suitability of using a Western research paradigm to study Māori. A written letter of support was supplied to accompany the ethics application. The researcher had a collegial relationship with Nga Tapuhi Whakawhanau (Wellington) a group made up of Māori and Pacific midwives who also provided support for the study application to The Ministry of Health. Ongoing consultation was made available by this group during study development, execution and booster sampling phases.

\subsection{FEEDBACK PROVISION FOR PARTICIPANTS}

Participants were invited to tick a box on the consent form they signed, whereupon a summary of results would be made available on study completion, if they desired. Recruiting midwives were also given the option of receiving a summary report. This will be made through posting on a website. Respondents and providers will be contacted via email or post of this upon finalisation and acceptance of the thesis.

\subsection{QUESTIONNAIRE DESIGN}

The quantitative phase of the research was the first stage in which measurement of the conceptual framework, its dimensions, sub-domains and variables was undertaken. In this section, an overview of the quantitative procedure is given, general objectives of the design, questionnaire design, survey distribution and data collection. A self-report questionnaire was used in order to gain understanding of the phenomena of the Transitional Heath Lifestyle of Pregnancy and explore the research questions within the framework of a psychographic type tool. Variables integral to each dimension of the framework were measured.

\subsubsection{Tool development}

This section outlines in detail the different stages in the development of the quantitative survey tool, which was designed specifically for this survey.

The questionnaire was structured into divisions and led respondents in a parsimonious and logical way, thereby providing a sense of order and progress. To achieve this, there was a small amount of re-ordering of variables from the conceptual framework so that the sequence was easier for the respondents. The questionnaire was developed through several iterative processes. 
For the purposes of this study, and using Wolburg and Pokrywczynski's (2001) classification of psychographic approaches as a guide, the aims of this study fit a general but context specific lifestyle approach. This means that respondents are classified into relatively homogenous groups to form a typology. Even though the focus of the study is health specific, some general AIO questions are relevant. Vyncke's (2002) suggested inclusion of media use, for instance, is useful, given the study's objective of improving health communication. Rodgers et al. (2007) also advocate the inclusion of media variables, not typically used in health segmentation, as they have been shown to be predictive of health behaviours such as 'diet and exercise'.

Psychographics is taken to be the operationalisation of the lifestyle construct, or the mechanism by which lifestyle is measured (Wedle \& Kamakura, 2000; Bruwer, 2002). This takes into account the diversity and range of different psychographic approaches, whilst keeping lifestyle defined at the construct level. A combined approach that incorporates values, AIO statements, media and related dimensions, especially health, are used in this study, consistent with approaches used by other authors (see for example, Vyncke, 2002).

Because the focus of this study is context specific, several customised items and scales measuring the pregnancy context and health were also imported and incorporated. This narrowing did not widely restrict the inclusion of variables that would appear in more general psychographic studies, if appropriate. For instance, the value 'concern for physical appearance' would be very useful in a study of pregnant women as it may well relate to attitudes toward changing body shape and weight gain.

The questionnaire was designed following the general format of psychographic studies and contained many AIO type questions. As this psychographic study was context specific, the instrument was constructed specifically for this study. In order to capture all the information that was desired from respondents, the questionnaire could be considered lengthy. Psychographic surveys however conventionally use large questionnaires (Wells, 1975). However, a degree of rationalisation and reduction was necessary to avoid respondent fatigue and abandonment. 
After the initial structure had been determined, informal interviews sourced from personal networks were undertaken with three pregnant women, whose characteristics were similar to the eventual desired target sample. This process provided generation and background for items, specifically item wording regarding health activities (and behaviours) undertaken in pregnancy for variables within the conceptual framework. This also provided a means of ensuring that all significant factors regarding health in pregnancy had been captured by the questions.

During the first stages of questionnaire development, several studies were consulted for questionnaire construction. Shaw (2004) conducted a study on a pregnant New Zealand sample. Some of the scales used by Shaw were relevant to this study, whilst others were not, as Shaw's study focused predominantly on self-efficacy and labour and birth outcomes, rather than pregnancy.

The SPARC 'Obstacles to Action' study (2003) was used to inform the questionnaire design. The SPARC questionnaire had been administered in New Zealand (although to a general non-pregnant market) and boasted a $60 \%$ response rate, despite its length. The SPARC questionnaire provided some items and also proved useful in terms of layout and formatting style. The 'Obstacles to Action' study is based on the original work of Maibach et al. (1996), notable for their original work in The Healthstyles surveys, a large study conducted regularly by Porter Novelli in the United States. The Healthstyles questionnaires are proprietary and were not available for consultation for this study. The SPARC study investigated exercise and nutrition, sourced from The Healthstyles instrument, with some modifications (for instance, appropriate wording for New Zealanders).

As the questionnaire came into form, several more revisions were undertaken. In the second stage of construction, four 'experts' (health professionals) were asked to review the questionnaire, particularly in regard to whether the wording of questions was appropriate and accurate for the context. This resulted in some change of wording (for instance, 'deliver' to 'give birth') and a further clarification of correct contextual phrasing. Guidance on Māori (wording of items and inclusion of any important subject matter was also sought from a Māori midwifery group and a Māori business academic). 


\subsubsection{Considerations specific to the context}

There are several differences between pregnancy and other research models of health that limit the importation of existing scales and measures directly from other health psychographic studies. In pregnancy, many of the usual health dimensions broadly stand, but there are some unique factors that require specificity. Firstly, although the present mode of healthcare for pregnancy involves close surveillance in a health system, it is an altered body state and not a disease. Being a younger demographic, an overwhelming percentage of women are healthy. Pregnancy is also a temporary state involving many alterations to physical structure and function that are peculiar to pregnancy only. Finally, the pregnant women must make health decisions directly impacting two people (the developing baby and herself).

Additionally, in New Zealand, the Lead Maternity Carer (LMC) and midwifery model of care means that the women are theoretically given the responsibility of selection and choice on the provider to choose. This situation is very different to most other countries in the Western world, and models of healthcare. Therefore, pregnant New Zealand women are exposed to a much different system of care than in other countries and this meant that construction of questions needed to capture this to ensure understanding.

\subsubsection{Item and scale generation}

This section contains the process by which items and scales were generated. Scale development procedures are as outlined by DeVellis (2003). Because of the abundance of existing scales, many of which have had extensive psychometric testing in various settings, these were used wherever possible. The use of existing measures enabled measurement to be undertaken with a good degree of confidence, with testing for reliability and validity testing kept to a minimum. Villani and Wind (1975) comment on the popularity of this process:

...the concept of traits or factors that can be quantitatively measured has led to the development of virtually hundreds of personality scales by psychologists. In the search of relationships between personality and consumer behaviour, marketing researchers have frequently used one of these many personality trait measurement instruments. These instruments have been used in two major ways: 1) in totality in their original form; 2) in sections and modified versions. (p. 223) 
However, there is some criticism of using unmodified scales, the main one being that a scale is designed for use in a context specific situation. For example Kassarjan (1971) cites:

the variables that lead to the assassination of a president, confinement to a mental hospital or suicide may not be identical to those that lead to purchase of a washing machine, a pair or shoes or chewing gum. ( $p 415$ ).

This criticism has been a consideration and, along with the increased popularity of using situation specific measures of personality in research (Sagrestano, 1999), led to the use of modified scales in this study, where deemed appropriate.

Kassarjian (1971) cautions against modifying scales stating "items are taken out of context, words are changed, items are arbitrarily discarded, and the test is shortened drastically" (p. 415). Villani and Wind (1971) tested the notion of reliability and validity of modified instruments 'holding'. For instance, scales developed on university students, or patients in a psychiatric hospital may not hold in a general population, particularly post-modification of the scale. However, Villani and Wind (1975) found encouraging results. Their study indicated that modified instruments can be reliable measures of the conceptual traits measured by other instruments:

This study indicated that personality instruments are relatively robust to major modification. Even though the modified instrument were considerably shorter, used different measurement scales and the wording of all the test items was not the same, the reliability of two out of three examined in the study tended to be within acceptable regions. (p. 237)

Villani and Wind (1971) concluded with the following recommendations: "most appropriate modified trait measures would therefore be one which preserves as much as possible, the measurement scale and weight procedure testing scale of the original instrument". They found that reliability measures "did not vary greatly or in any systematic manner depending on the item selection" (p. 238).

Two adjustment procedures were found to lower the inter-correlation of the modified trait measures. Firstly, the adoption of a measurement procedure in the modified instrument that differs conceptually from the original one (for example, forced choice to an indifference point scale) and secondly, weighting of an 
individual item response in the computation of a different trait score for the modified test than was done in the original test (Villani and Wind 1971 p. 238).

With the former in mind, the procedure and cautions taken to modify existing scales followed those recommended by Villani and Wind (1971). Conceptual construction was kept the same (including a balanced and ration reduction of subconstructs in the scale) and the measurement procedure kept the same (that is, likert scales were retained). In some cases the point numbers were increased; that is, an original 5 point scale increased to a 7 point scale. Wording of items was also changed to be more situation specific. All scales, directly imported or modified, had initial Cronbach alphas of 0.7 or above, except in a few instances, where a Cronbach alpha of between 0.65 and 0.69 was deemed to be acceptable.

In some cases existing scales were condensed, due to the potential excessive length of the questionnaire or further tailored to meet the characteristics of this study's context and population. Sagrestano, Rodriguez, Carroll, Bieniarz, Greenberg, Castro et al. (2002) suggest that single-item self-report measures may be more appropriate for certain types of variables than for others. Some health measurement scales required modification as they had been generated from a disease prevention orientation and were not appropriate in their raw state for the context of pregnancy. Likewise, some scales had become dated, where societal shifts in attitudes had changed over time. Suitability of existing scales to the New Zealand maternity context also required consideration, particularly when many health management practices and attitudes are culturally embedded. Language and wording in a few cases had to be changed to be more appropriate for New Zealand. This particularly included changing wording ("Kiwi-ising") or a sentence that better reflected the current New Zealand healthcare situation. Suggestions made after consultation with Māori reviewers were also incorporated into revisions.

In regard to the length of some pre-existing scales, the number of items made full inclusion cumbersome and inappropriate for an already long questionnaire. Sometimes this also meant an inappropriate balance; for instance, a long scale (19 items) was used to measure a minor variable and compared with five items to measure a scale considered key. For some scales, one or two additional items were added or original ones replaced with more appropriate ones. For instance, one item was added to the familism scale to be more consistent with the cultural make up of Māori families and after consultation with Māori experts. Any changes 
made to items were made according to the level of appropriateness and how conceptually sound they were.

As well as scales used in other studies, qualitative studies were useful, especially those where pregnant women were the sample. Bondas and Eriksson (2001) contributed many suggestions for items. The analysis and aggregation of subthemes from respondents in their study, such as 'health as no longer taken for granted', 'changing health behaviour', 'promoting the health of the unborn baby', 'the changing body' and 'ill-health as part of being pregnant', were helpful in informing the formation of variables within sub-domains for this study. In some cases, direct quotes in their article were extremely poignant and strongly influenced the wording of an item. For example, Bondas and Eriksson stated:

I hated to be pregnant, ugh. I thought it was disgusting and I still think so. I was so mad when someone told me I was big. I hated my belly and I would do almost anything so that people wouldn't see that I had that big belly. (p. 829)

This quote provided useful background to the wording of body image and attitude toward weight gain items in this study.

Several additional sources were referred to as a means of informing wording of items or to become familiar with context (for instance, what classes are available for women). Articles from the popular press were used to inform general knowledge of the context. For specific measurement items, recommended publications were consulted (for instance, to measure components of a 'healthy diet' in pregnancy, the Ministry of Health pamphlet given to pregnant women was used as a guideline). This assured that the behavioural measurement items were consistent with the advice and recommendations given by authoritative sources.

\subsubsection{Type of response}

Response was mainly continuous interval nominal items and scales. For interval items and scales, 7 point Likert scales were used. Most items used disagree/agree anchors: strongly disagree, neither agree nor disagree, strongly agree. There were also nominal items and some filtering of questions. Demographic questions were positioned at the end of the questionnaire. Some questions had an option for 'other' and where respondents had marked that, they were asked to 'specify'. 


\subsubsection{Presentation and formatting}

The final questionnaire was an A4 booklet of 24 pages, stapled in the centre. The questionnaire has a photograph on the front cover of an 'attractive' naked pregnant abdomen (in a strip of four identical images graduated in gray scale). This image was concept tested amongst women in the respondent demographic and was the one chosen from amongst other possibilities. The final image was also referred to Māori for approval, given that it showed a naked abdomen and not a whole person. Approval was given. The questionnaire was formatted and styled to make it as appealing to potential respondents as possible.

The questionnaire was broken into sections, which loosely followed the conceptual framework. Breaking the questionnaire into sections provided a logical flow through and also helped give a feeling of progress as one section was completed. In keeping with recommendations of minimising the impact of 'threatening' questions (Alreck \& Settle, 2004), the demographic section was presented at the end of the questionnaire. The first page contained instructions. At the end of the questionnaire the respondent was thanked and instruction given on how to send the completed questionnaire back. Sections are listed below:

- Section A: You as a person

- Section B: Questions about becoming pregnant and being pregnant

- Section C: Attitudes and opinions about pregnancy health

- Section D: Obtaining and responding to information in pregnancy

- Section E: Health behaviours

- Section F: Feelings and emotions

- Section G: About yourself

General layout and formatting of questions was informed by the SPARC 'Obstacles to Action' study (2003). As this questionnaire had been a similar length and had been well received by the target respondent group, it was felt this in some way could be attributed to the clarity and organisation of respondent's passage through and overall presentation. At the beginning of each section, a short explanation of what the section covered was given.

Sections headings were bolded and grey on white shading, which made the pages look attractive as well as provide a clear demarcation between items. This proved beneficial as some pages had rather long sections of questions. As there was a potential for respondent fatigue and abandonment, comments were included to 
encourage completion at the key marker points of the questionnaire; for instance, at the half way point, respondents were reminded they are 'just over halfway through' and thanked for their continued contribution.

\subsubsection{Questionnaire refinement and verification}

In the first stage of pre-testing, the questionnaire was tested for content and face validity. Face validity refers to "whether an item or scale, on the face of it, reads as if it measures what it is supposed to" (Cavana, Delahaye, \& Sekaran, 2000, p. 212). Content validity refers to how well the questionnaire adequately covers the topics that have been defined as the relevant dimension or dimension (Cooper \& Emory, 1995). In order to check content validity the process undertaken by Kaplan, Bush \& Berry (1976) was followed. Leeuw (2008) suggests that pretesting is the only way of assuring that the questions as written do communicate to respondents as intended and that the respondents will be able to answer the questions The questionnaire was therefore tested for understanding, readability, tolerance of length and that instructions were clear and any ambiguous questions identified.

In order for each item to remain in the final scale or as a single item, it has to be acceptable to the reviewers. This ensured the semantic meaning of each questionnaire item was ascribed to its respective dimension. The questionnaire was given to three academic colleagues from Victoria University and four midwives from personal networks who were practicing in the field. Midwives ensured that terminology was correct for the current New Zealand service setting. The questionnaire was then given informally to six women who were either nulliparous and pregnant, or had recently given birth to their first child within the past six months and were within the researchers personal network. One of the women in this group was Māori.

This phase was considered useful as it allowed a retrospective review of the appropriateness and value of the questions. On completion of the questionnaire, a follow-up informal feedback session was conducted with each woman individually, to provide feedback on the ease of the questions, confusion over questions and questions that may be considered offensive or controversial. Gathering feedback from questionnaires in this way is especially advantageous as it identifies any problems before the questionnaire is distributed to the sample proper. If there is difficulty understanding statements, they cannot be clarified in a self-administered questionnaire (González \& Bello, 2002). 


\subsubsection{Pilot Testing}

Finally the questionnaire was formally pilot tested with currently pregnant women for content and face validity and to provide further opportunity for feedback and general impressions of the questionnaire. The women were drawn from personal networks and midwifery contacts.

Twenty-two pilot test respondents, who were similar to the proposed targeted respondent group, and who were pregnant or had given birth within the past six months, were included in this phase. Including women who had given birth was considered advantageous, as they would be familiar with many of the sub-themes under investigation and therefore would be likely to raise some potentially useful issues, drawn from experience. Within the group, two respondents were Māori, one Chinese (New Zealand born) and a respondent who had recently arrived from the United Kingdom. Whilst this did not provide an extensive, cross-cultural testing of the survey tool, it was considered adequate. In addition, one respondent was 16 years of age (also Māori). One woman was anticipating a home birth.

The questionnaires were easily understood and able to be completed within the sixty minutes suggested. As a result of feedback, there were three items modified to more reflect the current New Zealand maternity care arrangements and terminology. Two respondents felt that the questionnaire was long (it took on average fifty minutes to complete) but if they broke it up it was felt to be easily achievable. This was added as an instruction. The questionnaire was also felt to be suitable by the Māori respondents.

\subsubsection{FINAL QUESTIONNAIRE MEASURES}

The following section explains the operationalisation of dimensions and the final measures for constructs/attribute sets and the variables within them. Presentation order follows the conceptual framework outlined in Chapter 3. Because of the large number of variables included in a study of this nature, the variable specifications are presented in tabular form at the end of each subdomain discussion. Definitions for variables have been provided in Chapter 3, so are not repeated here.

Each sub-section is entitled by the sub-domain on which discussion follows. Within the text, constructs and attribute sets are shown in bold italics. Variables are contained in double quotation marks and items and scales in single quotation 
marks. Items and scales are differentiated also, with scales italicized. The original source of scales/items and an indication of any treatment applied to original authors' work is also given. In the tables parentheses in the Item/scale column state the number of items and also whether the scale is new or existing. An (E) denotes the scale was existing, an (N) a new scale. Unless specified, all interval items were Likert scales, measured with a 7 point response.

\subsubsection{Socio-demographic}

"Age" was measured as a nominal single item, asking their years. "Ethnicity'"options were derived from the SPARC Obstacles to Actions study (2003) groupings used and were therefore reflective of the ethnic make-up of New Zealand population at the current time. More than one option could be selected. For European New Zealanders, the classification 'Pakeha' was used. "Household income" rather than individual income was sought as it was thought that most respondents would be living in a dual income household. For "Employment", 'Usual occupation', 'Employment status' and 'Leave from work plans' were nominal items. "Living situation" was made up of nominal items to address measurement of social support structures, parenting responsibilities (for example, for partner's children) and access to resources. "Domicile" was a single item broken into categories by small town to city. There was not a measurement for nominating the nearest town or city centre. "Media use" questions were primarily researcher generated but informed by Vynkce (2002).

Table 4.1 Socio-Demographic Measures*.

\begin{tabular}{|c|c|c|c|c|}
\hline Q no & Construct and its Variables & $\begin{array}{l}\text { Item } \\
\text { /scale }\end{array}$ & Measure & Anchors \\
\hline G1 & Age & Item & Nominal & Years \\
\hline G2 a-k & Ethnicity & Item & Nominal & Multi-option \\
\hline \multirow[t]{2}{*}{ G13 } & Household income & Item & Nominal & Select option \\
\hline & Employment & & & \\
\hline G11 & - 'Occupation' & Item & Nominal & Occupation \\
\hline G12 & - 'Employment status' & Item & Nominal & Select option \\
\hline G14 and 15 & $\begin{array}{l}\text { - 'Leave from work } \\
\text { plans' }\end{array}$ & Items (2) & Nominal & Weeks \\
\hline \multirow[t]{2}{*}{ G6 } & Education & Item & Nominal & Select option \\
\hline & Living situation & & & \\
\hline G3 & - $\quad$ Time in New Zealand & Item & Nominal & Years \\
\hline G4 & - Language & Item & Nominal & Language \\
\hline G7 & - $\quad$ Living arrangement & Item & Nominal & Select option \\
\hline G9 & $\begin{array}{l}\text { - Contact with father of } \\
\text { baby }\end{array}$ & Item & Nominal & Select option \\
\hline
\end{tabular}




\begin{tabular}{|c|c|c|c|c|c|}
\hline G10 & $\begin{array}{ll} & \text { Responsibility } \\
& \text { children }\end{array}$ & for & Item (2) & Nominal & Select option \\
\hline G5 & Domicile & & Item & Nominal & Select option \\
\hline A5 a-k & Media use & & Items (11) & Interval & Frequency \\
\hline
\end{tabular}

*All item researcher generated and informed by SPARC obstacles to Action study (2003)

\subsubsection{Pregnancy Circumstance}

This section contains measurement of the three constructs: Feelings about the pregnancy, Pregnancy dates and Other experiential factors.

\section{Feelings about the Pregnancy}

This construct comprised of four variables, which aimed to address how the respondent felt at the time of first awareness and then feeling currently about the pregnancy. "Pregnancy intendedness" was informed by D'Angelo et al. (2004). The second variable, "Original intentions for the pregnancy" and the third, "Evaluation of pregnancy worth" were modified from the Attitude to Pregnancy question by Gurung, Dunkel-Schetter, Collins, Rini, \& Hobel (2005). The final variable in this construct; "Rating as a life event", was scored on a continuum of 1 (worst)-10 (best). This item was based on the Appraisal of pregnancy measure by Huizink et al. (2002).

\section{Pregnancy Dates}

The Pregnancy Dates construct contained two variables: "Weeks now" and "First awareness". These variables determined how pregnant the respondents were, both at the time of completing the questionnaire and when they were first aware they were pregnant (by weeks of gestation). Both items were researcher generated.

\section{Other Experiential Factors}

Other Experiential Factors variables included "Level of risk" which was a filter question. Those respondents who specified they were high risk were forwarded to a following question and asked to indicate from a range of options why they were high risk. "Knowing the sex of the baby" and "Previous pregnancy" were single items. "Fertility assistance required' consisted of two questions. The first was a filter, where those who ticked yes to assistance where directed to another question asking for the type of assistance they had received. All the items in this construct were researcher generated. 
Table 4.2. Pregnancy Circumstance Measures.

\begin{tabular}{|c|c|c|c|c|}
\hline Q no & Construct and its Variables & $\begin{array}{l}\text { Item } \\
\text { /scale }\end{array}$ & Type & Anchors \\
\hline & Feelings about the & & & \\
\hline & Pregnancy & & & \\
\hline B1a-d & Pregnancy intendedness & Item & Nominal & 1 option \\
\hline$B 2 a-d$ & Original intentions & Item & Nominal & 1 option \\
\hline B3a-c & Evaluation of pregnancy worth & Item & Nominal & 1 option \\
\hline \multirow[t]{2}{*}{ B4 } & Rating as a life event & Item & Interval & $1-10$ rating \\
\hline & Pregnancy Dates & & & \\
\hline B8 & Weeks now & Item & Nominal & Weeks \\
\hline \multirow[t]{4}{*}{ B7 } & First awareness & Item & Nominal & Weeks \\
\hline & Other Experiential & & & \\
\hline & Circumstances & & & \\
\hline & Level of Risk & & & \\
\hline B12 & - Level of risk & Item & Nom/Filter & 1 option \\
\hline \multirow[t]{2}{*}{ B13 } & - $\quad$ Risk type & Item & Nominal & Multi-option \\
\hline & Knowing sex of baby & & & \\
\hline B9 & - Knowledge of sex of baby & Item & Nominal & 1 option \\
\hline $\mathrm{C} 100$ & - $\quad$ Attitude toward knowing & Item & Interval & Agreement \\
\hline B5 & Previous pregnancy & Item & Nominal & 1 option \\
\hline B6a-e & Fertility assistance required & Item & Nominal & Multi-option \\
\hline
\end{tabular}

\subsubsection{Pre-existing Influences}

In this section, respondents were asked to score the questions as how they normally feel, that is, when they are not pregnant. Their measures are almost exclusively questions in Section $A$ of the questionnaire. Tables at the conclusion of each sub-section summarise the scale and item specifications.

\section{Pre-existing health}

"Health as a value" was measured as a single item, existing from Dutta-Bergman (2003). "Health consumerism" was measured with a four item scale from DuttaBergman (2003) and comprised of three dimensions: reading, questioning and complaining (Cronbach alpha 0.85). The measure for "Pre-pregnancy health" was modified from the Health Consciousness scale of Dutta-Bergman (2003) (Cronbach alpha 0.72) and a Physical Health Rating scale (Cassidy, 2000) (Cronbach alpha, 0.84.). 


\section{Mastery and Achievement}

"Sense of mastery" was measured with a modified Mastery Index, a well known sociological instrument originally defined by Pearlin and Schooler (1978), measuring personal, social and structural processes (Cronbach's reliability coefficient; 0.74). The degree of "Feeling toward change", which could potentially influence the ability to embrace changes pregnancy brings, was researcher generated.

\section{Other Values, Traits and Beliefs}

"Trait anxiety" was measured using a reduced form of the State Trait Anxiety Inventory (Spielberger, 1983), as the unmodified form was considered too long for the questionnaire. This scale has a reported Cronbach alpha of 0.85 . "Universal values" was measured with Kahle's (1984) List of Values (LOV). "Time orientation" was a single item measure from Divine and Lepisto (2005). "Materialism" was measured with a single item used by Tilikidou and Delistavrou (2001). "Environmentalism" was measured with a three item scale modified from Tilikidou and Delistavrou (Cronbach alpha; 0.72). "Concern for physical appearance' was a sub-scale made up of Physical Concern items from the Vanity scale (Cronbach alpha; 0.91) developed by Netemeyer, Burton \& Lichenstein, (1995). The sub-scale consisted of five items for this study was reduced to three, with the removal of two items which seemed very similar to others.

The "Religiosity" measure consisted of a two item scale developed by Schieman et al. (2003) (Cronbach alpha; 0.83) was reduced to two items from four for this study. The level of "Superstition" a respondent felt they had was measured with a single item, similar to the approach used by Rudski (2003). Mowen and Carlson (2003) measured antecedents and consequences of superstition using sixteen constructs. However, this approach was considered too long for this questionnaire and a shorter scale was preferred. The "Cultural adherence strength" measure was informed by the work of Liamputtong et al., (2003).

"Female role" was measured with the scale developed by Sin and Yau (2004); Female role Orientation. The scale had a reported Cronbach alpha of 0.5, which Sin and Yau considered satisfactory within Exploratory Factor Analysis. Although this alpha was low and the scale development based on Confucian values, it was still regarded as appropriate for use in the study. However, several modifications were made to reduce the length of the scale and to better reflect the cultural and social environment of New Zealand. 
The "Familism" measure was modified from Steidel et al's (2003) Familism scale, with a Cronbach alpha of 0.83 . The scale has most commonly been utilised in Hispanic family contexts and there is no published use of it in a New Zealand context. Three items were added to the Familism scale. One item concerned the closeness of families relating at a 'distance' to each other'. The second was that 'friends can feel more like family'. A further item was added reflecting the importance of grandparents in childrearing following consultation with Māori. 
Table 4.3. Pre-existing Influence measures

\begin{tabular}{|c|c|c|c|c|}
\hline Q no & Construct and its Variables & Item/scale & Type & Anchors \\
\hline & Pre-existing health & & & \\
\hline A1 d & Health as a value & Item & Interval & Agreement \\
\hline A1 e-h & Health consumerism & Scale (4) (E) & Interval & Agreement \\
\hline \multirow[t]{2}{*}{$A 6 a-j$} & Self-reported health & Scale $(10)(E)$ & Interval & Quality \\
\hline & Mastery and Achievement & & Interval & \\
\hline A2 a-g & Sense of mastery & Scale (7) (E) & Interval & Agreement \\
\hline \multirow[t]{2}{*}{$A 1 b$} & Feeling toward change & Item & Interval & Agreement \\
\hline & Other Values, Traits and Beliefs & & Interval & \\
\hline A2 i-o & Trait anxiety & Scale $(7)(E)$ & Interval & Agreement \\
\hline A3 a-i & Universal values* & Items (9) & Interval & Importance \\
\hline A1 a & Time orientation & Item & Interval & Agreement \\
\hline A1 I & Materialism & Item & Interval & Agreement \\
\hline A1 $p-q$ & Religiosity & Scale (2) (E) & Interval & Agreement \\
\hline A1 m-o & Environmentalism & Scale (3) (E) & Interval & Agreement \\
\hline A1 i-k & Concern for physical appearance & Scale (3) (E) & Interval & Agreement \\
\hline A1 c & Cultural adherence strength & Item & Interval & Agreement \\
\hline $\mathrm{A} 2 \mathrm{~h}$ & Level of Superstition & Item & Interval & Agreement \\
\hline A4 a-e & Female role & Scale (5) (E) & Interval & Agreement \\
\hline A4 f-r, $p$ & Familism & Scale (13) (E) & Interval & Agreement \\
\hline
\end{tabular}

*9 point Likert measure.

Measures for the Life in Transition sub-domains (Transition Adaptation Adjusters and the Transitional Self) and their respective constructs are now discussed.

\subsubsection{Transition Adaptation Adjusters}

This section explains measures for the variables of the Transition Adaptation Adjusters constructs: Information Acquisition, Information Processing and Utilisation and Other Pregnancy Attitudes and Drivers.

\section{Information Acquisition}

This construct measured "Information seeking activity", "Information sources and usefulness" and "Antenatal classes". "Information seeking activity" was measured with a new scale; 'Information receipt interest'. This was informed by McKenzie, 2001 Deutsch et al., 1988; Dutta-Bergman, 2003; Maher, 2000). For "Information sources and usefulness", 'Ways of knowing' that a woman may place value on was informed by Root and Browner (2001). 'Usefulness of knowledge sources' items were informed by Aldoory (2001) and Dunn et al. (2003). Respondents were also asked how useful they considered each source. "Antenatal classes" items were researcher generated and covered the broad 
range of classes offered in New Zealand and type of topics most valued. Whilst most of the offerings are generic, some specialist classes are available (for instance, yoga and home birth classes).

\section{Information Processing and Utilisation}

"Information processing self-efficacy" measured the confidence respondents felt they had in their ability to receive, understand and use the information acquired. The measure for this variable was informed by Aldoory (2001) and Wu and Chung (2003). "Perceived information benefits and outcomes" was measured with a new scale, 'Perceived positive information outcome', which assessed beliefs about advantages of having and using information women have and was also informed by Aldoory (2001). Likewise, "Perceived difficulties and barriers" and "Changes due to information" were researcher generated but had been informed by Aldoory (2001). "Arranging maternity care" items were generated by the researcher and based on the current options available to women for care. Issues involved in arranging care were suggested by expert opinion as factors that impacted on a woman's choice and the satisfaction she felt with the choice she had made.

\section{Other Pregnancy Attitudes and Drivers}

Items from a qualitative study by Kornelsen (2002) and insights from Taylor (2000) informed the 'Affinity for technology in pregnancy" items. Five items were created that reflected the use of technology in pregnancy. It was hoped that these items might form a new sub-scale for the existing Health Locus of Control (HLOC) scale, following the suggestion of Wallston (2005), who has encouraged other empirical work to develop variations of the original HLOC scale.

In consideration of "Pregnancy Health Locus of Control", Wallston et al. (1978) constructed The Multidimensional Health Locus of Control, based on earlier work on Locus of Control by Rotter (1954). The Multidimensional Health Locus of Control scales are defined by Wallston (2005) as a 'family of instruments' that includes two "more or less equivalent forms $A$ and $B$ that are health-focused. These scales contain three six-item subscales: Internality (IHLC), Powerful Others Externality (PHLC) and Fate (chance) Externality (CHLC). For this study, The 'Fetal Health Locus of Control' scale (Labs \& Wurtle, 1986) was used, but required modification to adequately reflect the New-Zealand context. Cronbach alphas reported by Labs and Wurtle (1986) were IHLC; 0.88 , PHLC; 0.67 and CHLC; 0.83 . 
The "Pregnancy Health Effort and Motivation" variable consisted of two questions: a motivation and effort scale and questions regarding their level of concern about making changes. The 'Pregnancy health effort and motivation' scale was researcher generated. "Approach/avoidance" and "Achievement motivation" items were researcher generated. "Enablers and barriers to change" included items for measuring self efficacy for making health changes, the ability to practice cultural beliefs and enablers and constraints, such as time and money. "Reasons for health changes made" measured possible reasons for making changes that were welfare of self generated, response to others generated or welfare of baby generated and influence motivations. These items were researcher generated.

Table 4.4 Transition Adaptation Adjusters measures.

\begin{tabular}{|c|c|c|c|c|}
\hline Q no & Construct and its Variables & $\begin{array}{l}\text { Item } \\
\text { /scale }\end{array}$ & Type & Anchors \\
\hline & Information Acquisition & & & \\
\hline & Information seeking activity & & & \\
\hline $\mathrm{D} 1 \mathrm{c}, \mathrm{d}, \mathrm{f}, \mathrm{jandp}$ & - Ways of knowing & 5 item Q & Interval & Agreement \\
\hline D1a-b & $\begin{array}{l}\text { Information receipt } \\
\text { interest }\end{array}$ & Scale $(2)(N)$ & Interval & Agreement \\
\hline$D 2 a-x$ & $\begin{array}{l}\text { Information sources and } \\
\text { usefulness }\end{array}$ & 24 item Q & Interval & Usefulness \\
\hline \multirow[t]{3}{*}{ D4a-h } & Antenatal classes & 8 items Q & Nominal & Multi-option \\
\hline & $\begin{array}{l}\text { Information Processing and } \\
\text { Utilisation }\end{array}$ & & & \\
\hline & $\begin{array}{l}\text { Information processing self } \\
\text { efficacy }\end{array}$ & & & \\
\hline \multirow[t]{2}{*}{$\mathrm{DI}, \mathrm{n}$} & $\begin{array}{l}\text { - Information processing } \\
\text { self efficacy }\end{array}$ & Items (2) & Interval & Agreement \\
\hline & $\begin{array}{l}\text { Perceived information benefits } \\
\text { and outcomes }\end{array}$ & & Interval & \\
\hline \multirow[t]{3}{*}{$\mathrm{D} 3 \mathrm{c}, \mathrm{d}, \mathrm{f}, \mathrm{h}$ and D1h } & - $\quad$ Perceived positive & Scale (4) $(N)$ & Interval & Agreement \\
\hline & information outcomes & & & \\
\hline & $\begin{array}{l}\text { Difficulties and barriers } \\
\text { processing information }\end{array}$ & & & \\
\hline $\mathrm{De}, \mathrm{i}, \mathrm{k}, \mathrm{o}$, andD3e & $\begin{array}{l}\text { Perceived difficulties and } \\
\text { barriers processing } \\
\text { information }\end{array}$ & 5 items & Interval & Agreement \\
\hline $\mathrm{D} 1 \mathrm{~g}, \mathrm{~m}$, and $\mathrm{D} 3 \mathrm{~g}$ & $\begin{array}{c}\text { - 'Resistance to change' } \\
\text { Change as a result of information }\end{array}$ & 3 items & $\begin{array}{l}\text { Interval } \\
\text { Interval }\end{array}$ & Agreement \\
\hline D3a and b & $\begin{array}{l}\text { - Information change } \\
\text { result }\end{array}$ & Scale $(2)(N)$ & Interval & Agreement \\
\hline & Arranging pregnancy care & & & \\
\hline
\end{tabular}




\begin{tabular}{|c|c|c|c|c|}
\hline Q no & Construct and its Variables & $\begin{array}{l}\text { Item } \\
\text { /scale }\end{array}$ & Type & Anchors \\
\hline B10a-d & - $\quad$ Intended place of birth & Item & Nominal & 1 option \\
\hline \multirow[t]{4}{*}{ B11a-e } & $\begin{array}{ll}\text { - } & \text { Type of LMC } \\
\text { - } & \text { How care arranged }\end{array}$ & Item & $\begin{array}{l}\text { Nominal } \\
\text { Nominal }\end{array}$ & 1 option \\
\hline & i. How care arranged & Item & Nominal & Multi-option \\
\hline & ii. Issues in obtaining care & Item & Interval & Agreement \\
\hline & $\begin{array}{l}\text { Other Pregnancy Attitudes } \\
\text { and Drivers }\end{array}$ & & $\begin{array}{l}\text { Interval } \\
\text { Interval }\end{array}$ & \\
\hline \multirow[t]{3}{*}{ C1a-e } & $\begin{array}{l}\text { Affinity for technology in } \\
\text { pregnancy }\end{array}$ & Items (5) & Interval & Agreement \\
\hline & Pregnancy Health Locus of & & Interval & \\
\hline & Control & & & \\
\hline $\mathrm{C} 1 \mathrm{f}, \mathrm{h}, \mathrm{l}, \mathrm{m}, \mathrm{n}$ & - $\quad$ i Internal HLOC & Scale (5) (E) & Interval & \\
\hline$C 1 g, i, j, o, r$ & $\begin{array}{l}\text { - } \quad \text { External HLOC; } \\
\text { Powerful others }\end{array}$ & Scale(5) (E) & Interval & Agreement \\
\hline $\mathrm{C} 1 \mathrm{q}, \mathrm{s}, \mathrm{t}, \mathrm{u}, \mathrm{v}$ & $\begin{array}{c}-\quad \text { iii External HLOC: Fate } \\
\text { Preg health effort and motivation }\end{array}$ & Scale $(5)(E)$ & $\begin{array}{l}\text { Interval } \\
\text { Interval }\end{array}$ & \\
\hline$C 1 x, y, z$ and $b b$ & $\begin{array}{l}\text { - Preg health effort and } \\
\text { motivation }(N) \\
\text { - Level of concern }\end{array}$ & Scale $(5)(N)$ & $\begin{array}{l}\text { Interval } \\
\text { Interval }\end{array}$ & Agreement \\
\hline $\mathrm{C} 1 \mathrm{w}$ & i. Worry about what others think & Single item & Interval & Agreement \\
\hline C1aa & ii. Worry only if got sick & Single item & Interval & Agreement \\
\hline $\mathrm{C} 1 \mathrm{cc}$ & Achievement motivation & Single item & Interval & Agreement \\
\hline C1dd & Approach-Avoidance & & Interval & \\
\hline C1II & $\begin{array}{l}\text { i. Desire to be involved } \\
\text { in decision making }\end{array}$ & Single item & Interval & Agreement \\
\hline \multirow[t]{2}{*}{ C1ee } & $\begin{array}{l}\text { - ii. Rather not know if } \\
\text { something wrong }\end{array}$ & Single item & Interval & Agreement \\
\hline & $\begin{array}{l}\text { Enablers and barriers of heath } \\
\text { practices and change }\end{array}$ & & Interval & \\
\hline C1hh, ii, kk & $\begin{array}{l}\text { - Self efficacy for making } \\
\text { health changes' }\end{array}$ & Scale $(3)(N)$ & Interval & Agreement \\
\hline C1dd & $\begin{array}{l}\text { Ability to practice } \\
\text { cultural beliefs }\end{array}$ & Single item & Interval & Agreement \\
\hline \multirow[t]{2}{*}{ C3a-j } & $\begin{array}{l}\text { - Perceived barriers to } \\
\text { change or improvement }\end{array}$ & 10 item Q & Nominal & Multi-option \\
\hline & Reasons for health changes made & & & \\
\hline$C 2 a, d, e, f, g, j$ & $\begin{array}{l}\text { - } \quad \text { Self generated } \\
\text { - } \quad \text { Others generated }\end{array}$ & $\begin{array}{l}7 \text { items } \\
1 \text { item }\end{array}$ & $\begin{array}{l}\text { Interval } \\
\text { Interval }\end{array}$ & $\begin{array}{l}\text { Influence } \\
\text { Influence }\end{array}$ \\
\hline $\mathrm{C} 3 \mathrm{~h}, \mathrm{i}, \mathrm{k}$ & $\begin{array}{l}\text { Welfare of } \\
\text { generated }\end{array}$ & 3 items & Interval & Influence \\
\hline
\end{tabular}




\subsubsection{The Transitional Self (Physical)}

\section{Nutrition}

The Nutrition construct contained five main variables: "Nutrition attitudes", "Nutrition practices", "Reducing nutritional risk" and "Barriers to good nutrition".

"Nutrition attitudes" was made up of questions that included 'Good nutrition outcome beliefs', the extent to which respondents felt good nutrition would achieve particular pregnancy outcomes was informed by Slater and Flora (1991). Questions on 'Eating disturbance' and 'Nutrition and weight gain' were researcher generated. "Nutrition Practices" measured firstly the effort being put into achieving what respondents felt was good pregnancy nutrition: 'Good nutrition effort' (researcher generated) and 'Intake against guidelines', the intake of food group portions against recommended guidelines for pregnant New Zealand women (Ministry of Health, 2006). "Reducing nutritional risk"' asked respondents firstly the extent to which they were 'Avoiding risky food' and then secondly, their 'Folic acid intake' supplementation history'. "Barriers to good nutrition" contained 'Perceived barriers to good nutrition', items, made up of internal and external factors that could interfere with practicing good nutrition. Items for this question were modified from SPARC Obstacles to Action (2003), for a pregnancy context and wording also further informed by the 'Barriers to Health Eating' scale (Fowles \& Feucht, 2004).

\section{Exercise}

Exercise was made up of "Attitudes about exercise" "Exercise practices", "Barriers and interference to exercise" and "Rest and sleep patterns". "Attitudes about exercise" was made up of one question that scoped the beliefs about benefits brought about by exercise. "Exercise practices" contained a filter question asking if they had a planned regime for exercise, and if yes, who had designed that plan. Another items measured 'whether they followed exercise recommendations and guidelines, how often they exercised and thirdly, the activities they engaged in. For "Exercise Barriers" respondents were asked the extent to which pregnancy had interfered with their ability to exercise (a researcher generated 2 item scale) and then secondly, 'Barriers to exercise' (internal and external). These questions were researcher generated, informed by the SPARC 'Obstacles to Action' study (2003). "Sleep and rest patterns" consisted of two questions: first, whether 'Rest requirements had increased' and second, whether they felt their 'Sleep patterns 
had been disturbed' since becoming pregnant. "Sleep and rest" questions were researcher generated.

\section{Substance Use}

The Substance Use construct was made up of variables; "Tobacco use", "Alcohol intake", "Medication use" and "Illicit drug use". "Tobacco use" questions first filtered those who had not smoked tobacco cigarettes in the 12 months prior to the pregnancy Smokers who had both ceased and those who continued were led to a question with items regarding the motivation and beliefs that had influenced them giving up once pregnant or for continuing and their current smoking profile. Items for this section were modified from SPARC Obstacles to Action (2003) for a pregnancy context. The "Alcohol intake" variable included questions with a filter for alcohol use history, then a question regarding intake since pregnant (also a filter question). There was one item asking if pregnant women should drink alcohol. For those who continued to drink, they were led to a question regarding frequency and amount consumed on an average drinking occasion items "Medication use" contained two questions: first, a question about their attitude toward taken medication ( 3 items); second, a question asking whether they had taken certain medications whilst pregnant. Information was sought on usage of Over-The-Counter, homoeopathic and vitamins. The "Illicit drug use" item was sensitively worded to avoid potential respondent resistance to answering.

\section{Risk and Risk Reduction}

Risk and risk reduction consisted of three variables; "Dental hygiene"" "Pre-natal screening'" and "Environmental safety". The "Dental hygiene" item was researcher generated. "Pre-natal screening" was operationalsied as ultrasound scanning questions. "Environmental safety" questions were also researcher generated. The question regarding fear a respondent felt because of the threat of emotional or physical violence was worded sensitively, to cover the spectrum of violence that might be encountered.

Table 4.5. Transitional Self Measures (Physical).*

\begin{tabular}{|c|c|c|c|c|}
\hline Q no & Construct and its Variables & Item/scale & Type & Anchors \\
\hline & Nutrition & & & \\
\hline & Nutrition attitudes & & & \\
\hline E1.c, i, m & $\begin{array}{l}\text { - Good nutrition outcome } \\
\text { beliefs }\end{array}$ & Items $(3)^{*}$ & Interval & Agreement \\
\hline E1.d,f,l1 & - $\quad$ Eating disturbance & Items $(3)^{*}$ & Interval & Agreement \\
\hline
\end{tabular}




\begin{tabular}{|c|c|c|c|c|}
\hline Q no & Construct and its Variables & Item/scale & Type & Anchors \\
\hline $\mathrm{C} 1 . \mathrm{h}, \mathrm{j}, \mathrm{k}$ & $\begin{array}{l}\bullet \quad \text { Nutrition and weight gain } \\
\text { Nutrition Practices }\end{array}$ & Items $(3)^{*}$ & $\begin{array}{l}\text { Interval } \\
\text { Interval }\end{array}$ & Agreement \\
\hline $\begin{array}{l}\text { E1.a,b, } \\
\text { E2.g,h }\end{array}$ & - Good nutrition effort level & Scale $(4)(N)$ & Interval & Agreement \\
\hline$E 2 a-f$ & $\begin{array}{ll}\text { - } & \text { Nutrition } \\
\text { guidelines } & \text { against }\end{array}$ & Items (6) & Interval & Frequency \\
\hline E5 & $\begin{array}{c}\bullet \quad \text { Vegetarianism } \\
\text { Reducing nutritional risk }\end{array}$ & Item & Nominal & 1 option \\
\hline$E 2 i$ & - $\quad$ Risky food avoidance & Item & Interval & Frequency \\
\hline \multirow[t]{2}{*}{ E3 and 4} & - Folic acid intake & Items (2) & $\begin{array}{l}\text { Nom/ } \\
\text { Filter }\end{array}$ & 1 option \\
\hline & $\begin{array}{l}\text { Perceived barriers to good } \\
\text { nutrition }\end{array}$ & & & \\
\hline \multirow[t]{3}{*}{ E1.e,g. E.a-h } & $\begin{array}{l}\text { - Perceived barriers to good } \\
\text { nutrition }\end{array}$ & Items $(10)$ & Interval & Amount \\
\hline & Exercise & & Interval & \\
\hline & $\begin{array}{l}\text { Attitudes and knowledge about } \\
\text { exercise }\end{array}$ & & Interval & \\
\hline$E 7 . c, f, k, n$ & - $\quad$ Benefits & Items (4) & Interval & Agreement \\
\hline E7d & - Danger for baby & Item & Interval & Agreement \\
\hline E7i & $\begin{array}{ll}\text { - } & \text { Information sufficiency } \\
\text { - } & \text { Exercise Practices } \\
\text { - } & \text { Planning }\end{array}$ & Item & Interval & Agreement \\
\hline E8 & - i. Uses plan & 1 item & $\begin{array}{l}\text { Nom } \\
\text { /Filter }\end{array}$ & 1option \\
\hline E9a-f & $\begin{array}{l}\text { - ii. Source of plan } \\
\text { - Patterns }\end{array}$ & Items (6) & Nominal & Multi-option \\
\hline \multirow[t]{2}{*}{$E 7 j$} & $\begin{array}{l}\text { - i.Follows } \\
\text { recommendations }\end{array}$ & Item & Interval & Agreement \\
\hline & $\begin{array}{l}\text { - ii. Type of activities } \\
\text { Barriers and interference to } \\
\text { exercise }\end{array}$ & Items (9) & Nominal & Multi-option \\
\hline E7a-b & $\begin{array}{l}\text { - Perceived interference } \\
\text { level }\end{array}$ & Scale $(2)(N)$ & Interval & Agreement \\
\hline$E 7 . e, I, o, t$ & - Internal Barriers & Items (5) & Interval & Agreement \\
\hline E7. $m, q, r, s$, & $\begin{array}{l}\text { - External Barriers } \\
\text { Sleep and rest patterns }\end{array}$ & Items (4) & $\begin{array}{l}\text { Interval } \\
\text { Interval }\end{array}$ & Agreement \\
\hline E7h & - Rest requirements & 1 item & Interval & Agreement \\
\hline \multirow[t]{4}{*}{$E 7 g$} & - $\quad$ Sleep patterns & 1 item & Interval & Agreement \\
\hline & Substance-Use & & & \\
\hline & Tobacco use & & & \\
\hline & - $\quad$ Attitude toward & & Interval & \\
\hline
\end{tabular}




\begin{tabular}{|c|c|c|c|c|}
\hline Q no & Construct and its Variables & Item/scale & Type & Anchors \\
\hline C1pp & - $\quad$ i. Pregnant women & 1 item & Interval & Agreement \\
\hline C1mm & $\begin{array}{l}\text { - } \quad \text { ii. Others around } \\
\text { - } \quad \text { Smoking activity }\end{array}$ & 1 item & Interval & Agreement \\
\hline E13 & $\begin{array}{l}\text { - i. Activity } 12 \text { months } \\
\text {.prior }\end{array}$ & 1 item & Nom/Filter & 1 option \\
\hline E14 & $\begin{array}{l}\text { - ii. Smoking patterns since } \\
\text { pregnant }\end{array}$ & 2 items & Nominal & 1option \\
\hline E15a-g & $\begin{array}{l}\text { Influences over current smoking } \\
\text { choice } \\
\text { Alcohol use }\end{array}$ & 7 item Q & Interval & Multi-option \\
\hline $\mathrm{C} 1 \mathrm{jj}$ & $\begin{array}{l}\text { - Attitude toward alcohol in } \\
\text { pregnancy } \\
\text { - Alcohol intake }\end{array}$ & & Interval & Agreement \\
\hline E17 & i. Prior & 1 item & Nom/Filter & 1 option \\
\hline \multirow[t]{2}{*}{$\mathrm{E} 18,19,20$} & ii. Since & 3 items & Nominal & 1 option \\
\hline & Medication use & & & \\
\hline \multirow[t]{4}{*}{ C1. $K, p, n n$} & $\begin{array}{lll}\text { - Attitude } & \text { toward } \\
\text { medication } & \end{array}$ & 1 items & Interval & Agreement \\
\hline & - Medication taken & 1 item & Nominal & 4 options \\
\hline & Illicit drug use & 1 item & Nominal & 2 options \\
\hline & Risk and Risk Reduction & & & \\
\hline \multirow[t]{2}{*}{ C1gg } & Dental hygiene & 1 item & Interval & Agreement \\
\hline & Prenatal screening (scanning) & & & \\
\hline C1d & - $\quad$ Attitude toward scanning & 1 item & Interval & Agreement \\
\hline C16 & - Undertook scan' & 1 item & Nom/Filter & 1 option \\
\hline C17a-g & $\begin{array}{l}-\quad \text { Value of findings to self } \\
\text { Environmental safety }\end{array}$ & 7 item & Interval & Importance \\
\hline C1ff & - $\quad$ Safety belt in car & item & Interval & Agreement \\
\hline G8e & - Experience of violence & item & Interval & Agreement \\
\hline
\end{tabular}

*All continuous items measured on 7 pt Likert

\subsubsection{The Transitional Self (Psycho-social)}

The Transitional Self (Psycho-social) contained the constructs Support, Coping and Experiencing the pregnant body'

\section{Support}

Support consisted of 'Sources support' and 'Satisfaction with living environment'. 'Sources support' firstly, measured the 'importance of source support' and then secondly, the 'perceived level of support', the level to which they felt support had been received for each source. Both questions were modified from those of 
Sagrestano, Feldman, Rini, Woo, \& Dunkel-Schetter, (1999).) (1999). The third question asked respondents to rate the importance to them of different types of support: pregnancy and birth related, emotional and enjoyment of life and daily chores/financial. This question was researcher generated, with wording informed by Sagrestano, et al, (1999) and Gurung, Dunkel-Schetter,Collins, Rini, \& Hobel, (2005). 'Satisfaction with living situation' included factors such as transport, warmth and 'getting on with people in the house' and was informed by the SPARC 'Obstacles to Action' (2003) questionnaire.

\section{Coping}

'Anxiety' was measured by 'Pregnancy state anxiety' (PSA). This scale was modified for context fit from Rini et al's. (1999) Pregnancy state anxiety scale (PSA). Rini et al (1999) report a Cronbach alpha for the PSA as 0.72.and further informed by Gurung et al. (2005). Wording for the 'Life situation state anxiety' items were also informed by Gurung et al. (2005). For 'Emotions and reactions' two single item questions were used. The first asked if they considered themselves to be more emotional and the second addressed whether they felt they solved problems rationally. These were researcher generated. For 'Relaxation and stress reduction', activities used to relax were addressed with one question which listed possible options.

\section{Experiencing the Pregnant Body}

'Experiencing the Pregnant Body' contained the variables "Pregnant body image", "Appearance interest" and "Celebrity attraction". 'Pregnant Body satisfaction' measured satisfaction with their gravid body and was researcher generated. 'Weight concerns' contained a question on feelings about weight gain in pregnancy. This was distinct from the question on weight in the nutrition variable, as it was concerned with body image and weight gain, not nutrition and weight gain per se. "Appearance' interest" was measured with items. Measures employed for these three variables were all informed by DiPietro, Millet. Costigan, Gurewitsch \& Caulfield, (2003) during development.

The 'Celebrity attraction' scale measured the level of interest in celebrities' lives and the extent to which a respondent felt their interest influenced their behaviour or goals for body appearance in pregnancy. This scale was researcher generated. 
Table 4.5. Transitional Self measures (Psychosocial).*

\begin{tabular}{|c|c|c|c|c|}
\hline Q no & Variable & Item/scale & Type & Anchors \\
\hline & Support & & & \\
\hline & Sources support & & & \\
\hline F1a-i & - Importance from source & Items (9) & Interval & Importance \\
\hline$F 2 a-i$ & $\begin{array}{l}\text { - Level received from } \\
\text { source }\end{array}$ & Items (9) & Interval & Level \\
\hline \multirow[t]{4}{*}{ F3a-m } & - $\quad$ Type of support desired & Items (9) & Interval & Level \\
\hline & i. Pregnancy and birth related & Items (6) & Interval & Importance \\
\hline & $\begin{array}{l}\text { ii. Emotional and enjoyment of } \\
\text { life }\end{array}$ & Items (5) & Interval & Importance \\
\hline & iii. Daily chores/financial & Items (2) & Interval & Importance \\
\hline \multirow[t]{4}{*}{ G8a-h } & $\begin{array}{l}\text { Satisfaction } \quad \text { with } \quad \text { living } \\
\text { environment }\end{array}$ & & Interval & \\
\hline & $\begin{array}{l}\text { - Satisfaction with living } \\
\text { environment }\end{array}$ & items (7) & Interval & Multi-option \\
\hline & Coping & & & \\
\hline & Emotions and reactions & Item & Interval & Agreement \\
\hline $\mathrm{F} 4 \mathrm{e}$ & $\begin{array}{l}\text { - Changes in emotional } \\
\text { level }\end{array}$ & Item & Interval & Agreement \\
\hline \multirow[t]{2}{*}{$\mathrm{F} 4 \mathrm{q}$} & - $\quad$ Problem solving & Item & Interval & Agreement \\
\hline & Anxiety & & & \\
\hline F4a-h & - $\quad$ Pregnancy state anxiety & Scale $(7)(N)$ & Interval & Agreement \\
\hline F4.s-u & $\begin{array}{l}\text { - Life situation state } \\
\text { anxiety }\end{array}$ & Scale $(4)(N)$ & Interval & Agreement \\
\hline $\mathrm{F} 4 \mathrm{q}$ & $\begin{array}{c}\text { - Problem solving } \\
\text { Relaxation and stress reduction }\end{array}$ & item & Interval & Agreement \\
\hline \multirow[t]{2}{*}{ F5a-i } & Sources of relaxation & Items (9) & Nominal & Multi-option \\
\hline & $\begin{array}{l}\text { Experiencing the pregnant body } \\
\text { - Pregnant body image }\end{array}$ & & & \\
\hline F6. b, f,v,h,a,t, & i. Pregnant body image & Scale $(6)(N)$ & Interval & Agreement \\
\hline F6.m-s & $\begin{array}{l}\text { ii. Weight concerns } \\
\text { - Appearance interest }\end{array}$ & Scale $(7)(N)$ & Interval & Agreement \\
\hline F6.cd, g,i,k,l,l & $\begin{array}{l}\text { i. Appearance interest } \\
\text { - Celebrity attraction }\end{array}$ & Items (6) & Interval & Agreement \\
\hline F6.e,j,u & i. Celebrity attraction & Scale $(3)(N)$ & Interval & Agreement \\
\hline
\end{tabular}

*all continuous items measured on 7pt Likert

\subsubsection{Transition success}

'Transition Success constructs' included "Wellbeing" "Relationship with the Baby" and 'Orientation to the Future'. As subjective wellbeing indicates a successful transition course (Schumacher \& Meleis, 1994), "Perceptions of Wellness" was measured with a scale that reflected the dimensions of wellness in pregnancy. This was developed by the researcher. In this construct, the variable "Maternal- 
fetal attachment" was operationalised with a modified version of Müller's (1994) 'Prenatal Attachment Inventory' (Cronbach alpha 0.81). "Orientation to the future" contained two variables, each containing a single item that reflected a positive transition signal and forward progress. The wording was informed mainly by Mercer (2004), Schumacher and Meleis (1994) and Deutsch et al, 1998).

Table 4.7. Transition Success measures*

\begin{tabular}{|c|c|c|c|c|}
\hline Q no & Variable & Item/scale & Type & Anchors \\
\hline & Wellbeing & & & \\
\hline \multirow[t]{2}{*}{ B18a-f } & Perception of wellness & Scale(6) (N) & Interval & Agreement \\
\hline & Relationship with the Baby & & Interval & \\
\hline \multirow[t]{2}{*}{$F 4 j-p, r$} & Maternal/fetal attachment & Scale(9) (E) & Interval & Agreement \\
\hline & Orientation to the Future & & Interval & \\
\hline $\mathrm{F} 4 \mathrm{w}$ & Leaving childless days behind & $\operatorname{Item}(1)$ & Interval & Agreement \\
\hline $\mathrm{F} 4 \mathrm{x}$ & Looking forward to new life stage & Item(1) & Interval & Agreement \\
\hline
\end{tabular}

*all continuous items measured on 7 pt Likert

\subsubsection{SURVEY IMPLEMENTATION AND DATA COLLECTION}

This section describes the procedure undertaken to gain access to health care providers who would distribute the questionnaire to eligible women throughout the targeted collection sites, along with how best return could be maximized. Sampling issues and inclusion criteria are also discussed, along with rationales. Following this, the response rate calculation is presented. There was also a booster collection phase to capture an increased number of Māori and Pacific women which will be discussed in the final part of this section.

\subsubsection{Access arrangements}

Access to respondents in almost all cases was via health professionals who were Lead Maternity Carers (LMCs): midwives, specialist obstetricians and District Health Board staff (primary and low risk). Access via health professionals was optimal as it assumed the greatest potential for a representative sample in terms of demographics, risk and pregnancy circumstance. In one case, access was obtained through a childbirth education organisation.

Overwhelmingly LMCs approached were very accommodating. In regards to declines, of the five DHBs approached, one large hospital declined due to the amount other research commitments and one other hospital failed to respond to written and phone contact. Four midwives of the 43 approached failed to respond to initial phone messages. All midwives reached were agreeable to assist. All 
medical staff approached $(n=6)$ in GP or private practices responded favourably. The access approaches to each practitioner group are outlined below. All LMCs were assured that there would be no demand on their resources, other than a few minutes of their time introducing the study to clients.

\section{Independent midwives/Antenatal education provider}

In order to obtain a list of midwives practicing in New Zealand, The New Zealand College of Midwives (NZCOM) was approached. This was declined by them. The NZCOM was helpful, however, in allowing the purchase of advertising space in their magazine distributed to its membership (although this resulted in a response from two providers only). A Wellington branch meeting was also attended in the early stages of data collection to raise awareness of the study and build networks.

In order to access midwives in Independent Practice, direct contact was made; in the first instance, this was most often a phone call. In the initial stages, the researchers own networks were used. Some cold calling was also undertaken. Midwives approached were very helpful in offering names of other midwives and through a process of snowballing, a list of potential midwives to be approached was obtained. As most of the researchers networks were in Wellington, this was where most access was organised, although a good level of access was achieved over the country and in both rural and urban centres.

Many midwives worked with two to four other midwives in a range of practice structures, from simple informal arrangements, providing cover and support for each other, to more formal practice structures. This situation proved to be helpful in widening the net of potential contacts. Almost all consenting midwives contacted were also agreeable to take additional questionnaires to hand on to the other midwives they worked with. Midwives could also be working with a woman who was under the care of a private obstetrician, thereby giving potential access to women in 'shared care' arrangements.

Once initial contact had been established and opportunity to proceed to the next stage of LMC consideration had been achieved, a questionnaire pack was delivered or posted, along with written information. The study information sheet contained information already often discussed in the initial contact, but it formalised the process and also provided a reference point. Once an LMC had considered the contents and given their consent to be involved, an indication 
would be obtained as to how many questionnaires they felt they could distribute in the nominated data collection period (predicted on their average first births per month).

\section{General practitioners/Private obstetricians.}

A direct approach was made to these practitioners, in the same way as Independent Midwives were approached. Doctors accessed were mainly in the Wellington region.

\section{District Health Boards and Primary Health Organisations.}

Approach to DHBs (for access to both primary and high risk clinics) and Primary Health Organisations (PHOs) required application and consideration through their research centres. Once an access agreement had been agreed and signed off, the appropriate documentation was forwarded to the chairperson of The Multiregion Ethics Committee for approval and alteration to the existing ethics cover. Where access was denied into a DHB, the reasons given were an existing heavy research load in the relevant departments at the time, or that staff were considered too busy. Access to these organisations was often a complex process. Access to all types of LMCs often included a personal meeting and presentation.

\subsubsection{Sampling design}

The study applied a non-probability sampling approach (Malhotra, 2007; Cavana et al., 2001). In order to obtain valuable research findings within the shortest time possible, and in a cost-effective way, the study focused on a convenience sample of those who met the criteria for inclusion. However, it was important to ensure that as wide a range possible of ethnic groups were sufficiently represented within the sample. The guarantee of the representation of these ethnic minorities enabled a high precision of the sample being close to the population characteristics as possible (Cavana et al., 2001). For two of these groups a quota sample was sought, a second stage of booster sample was carried out to obtain more Māori and Pacific women.

\section{Inclusion criteria}

The following inclusion criteria were applied: pregnant, nulliparous, 20-36 weeks gestation and English language skills sufficient to answer the questionnaire. There were no age restrictions or restriction on risk status. These criteria are discussed below. 


\section{Pregnant}

As respondents were accessed through their Lead Maternity Carer, it was a given that they were pregnant.

\section{Nulliparous}

The study recruited nulliparous (no previous live birth) women. It did not exclude those with previous pregnancies that ended prior to 20 weeks. It would have been counterproductive to search for primigravid (not their first pregnancy) women. This would have eliminated a large number of potentially appropriate respondents, as a significant number of nulliparous women have had a pregnancy loss before 20 weeks gestation, through induced or spontaneous abortion.

Women who had had a previous birth after 20 weeks were excluded as the study aimed to investigate first time transition. The demarcation of 20 weeks was applied rather than a viable birth (of say 23 weeks) cut off as it was considered that after 20 weeks, a significant amount of experience would have been achieved and potentially influenced reporting in the questionnaire.

\section{0-36 weeks gestation}

These weeks of gestation were regarded as the most researchable as it is a time when, for most women, the early problems of pregnancy have passed and therefore not likely to be unduly influential when making responses. If the early weeks of pregnancy were canvassed, it would be very possible that the early problems of pregnancy (for example, morning sickness and fatigue) may have confounded a fair appraisal of health status and current wellbeing (although there were questions relating to early pregnancy, where recall would have been required). Additionally, it was anticipated that by 20 weeks a fair amount of pregnancy, and therefore transitional status, had been experienced that could be recounted.

A cut off of 36 weeks gestation was decided upon as after this period some women find pregnancy more difficult due to structural factors and discomforts, such as tiredness and pressure from fetal descent. As they await the onset of labour, their focus may also change to become introspective. Recruiting within the gestational period was undertaken by LMCs filtering the distribution of questionnaires. At times a respondent might have taken some time returning the questionnaire and give a gestation a little beyond 36 weeks. For these respondents, it was assumed that distribution and engagement had been 
undertaken within the inclusion criteria and the questionnaire was therefore included.

\section{Literacy}

Because of the need for a reasonable literacy standard in order to complete the questionnaire, midwives were asked to avoid offering questionnaires to those with a poor grasp of English (written and oral). Due to budget constraints, there was no translation possible.

\section{Additional considerations}

Locality

Women from urban, town and small town, localities were sought. A greater percentage of distribution was undertaken by LMCs in the wider Wellington region. This is reflective of the researcher's networks and not any intentional bias. Diversity of 'planned place of birth' was also sought, particularly home versus hospital birth.

\section{Representation versus convenience}

The sample was essentially a convenience sample of women who met the inclusion criteria, due to the complexities of accessing the respondent base. However, care was undertaken during networking and distribution of questionnaires to LMCs to ensure the most feasible and appropriate distribution to a sample that would generally represent the makeup of nulliparous births in New Zealand. For a sampling guide, 'Report on Maternity' documentation (Ministry of Health, 2004) was consulted to maximize the possibility that adequate power would be achieved to make generalisations from the prospective sample.

\section{Sample size}

The intention was to recruit a maximum of 1500 women within the inclusion criteria. Based on a $20 \%$ response rate, this would give 300 returned questionnaires, a minimum number needed for analysis. It was estimated that on average, each midwife would have 12 clients within the 20 to 36 week category, estimated on the average workload of 4 births a month (although there was no distinction to the proportion of first versus subsequent birth bookings a midwife would hold possible). 


\subsubsection{Distribution}

Distribution was a two stage process. In regards to LMCs, once consent and access was obtained, questionnaire packs were delivered either by post or personally. The number of questionnaire packs distributed to LMCs depended on the size of their practice and the number of bookings that were likely over the collection period. The average number of packs taken by a midwifery practice with two to three midwives was 24, for an individual 12 and for an DHB 80. In the delivery was also a distributor instruction sheet in case of hand-on to others. Care was taken to give consistent instruction. Following that, two to three phone calls were made to each contact LMC at regular intervals, in order to clarify issues, ascertain how distribution was proceeding and to keep them motivated.

Distribution to respondents was a staggered process and occurred over a number of months. The option determined best by practitioners was that the questionnaire pack be offered at a routine antenatal visit when the client fell into the inclusion criteria. An option of provision of postage material by the researcher was felt less convenient by practitioners than the former option. If the client accepted the questionnaire pack, they then took the package away to open, read and consider.

Completion and return by post was at a place and time suitable to them. Taking the questionnaire home for self-administration and completion in their own time increased the likelihood of well considered opinions, better disclosure and privacy. However, this did mean a potential problem of not being able to get clarification on instructions from the researcher of distributor (Leeuw, 2008) and added length and extra process to the response chain.

Each respondent questionnaire package for potential respondents contained an information sheet, questionnaire, consent form for signing, incentive (pen) and postage paid return envelope. Full confidentiality was assured and consent forms and questionnaires were also separated when opening the returned questionnaires, so no matching of consents forms and questionnaires was possible. Respondents were given full assurance that any decision made to participate or not would have no impact on their healthcare provided by their LMC and that their provider would not be in receipt of any information as a result of their participation. In addition, the consent form contained an option of receiving a future summary of findings and to go into the draw for one of two $\$ 100$ retail vouchers. This meant that respondents also provided a postal or email address. 
Respondents returned the completed questionnaire by mail, along with their consent form in the postage paid envelope provided.

\subsubsection{Changes within the external environment}

As the data collection period ran over several months, changes to the external environment occurring during that time were monitored, in case these influenced respondent answers. In order to do this, the researcher searched online New Zealand media databases for articles with key words such as midwifery, pregnancy, maternity. Throughout the period there was not infrequent media attention, particularly in newspapers regarding the situation of obstetric services in New Zealand. Attention was given to Health and Disability decisions regarding the professional management of cases by midwives that had resulted in less than ideal outcomes on approximately four occasions in the Wellington mass media. (newspapers and as television news items). Shortages of midwives were also reported in the media on three or so occasions in Wellington. There was one television news item discussing Government moves to fortify bread with folic acid, which at the time of finalising the thesis discussion had not been implemented. As questionnaires had been consecutively numbered on return and data entry had occurred in numeric order, it was possible to assess any changing patterns or trends in general response as a result of changes to the external environment. None were observed.

\subsubsection{Follow up letters and contact}

Respondent follow up letters were included in the original drop off for distributors to give to women who had taken a package away at a previous date. As the respondent was in regular contact with their provider, it was suggested the reminder letter be given to the client at their next visit, after at least a two week interval.

Questionnaires were distributed to LMCs as networking progressed, for a period of six months. Return of questionnaires was kept open for a further six months after that, when it became apparent that distribution on to women would take longer than had been thought.

A longer than projected data collection period occurred due to the slower than originally predicted distribution of questionnaires. The reasons for this seem to be threefold. Firstly, networking and gaining access took a longer time than originally thought. LMCs were found to be extremely approachable overall, but 
nevertheless, dates put forward for meetings and consideration periods needed to be fitted in around pre-existing obligations and client demands. Secondly, LMCs could not predict the ratio of bookings for first births compared with second or subsequent births that would occur during the specified data collection period. Some midwives reported only booking in 'multips' (second or more birth) over the period. Thirdly, it became evident on follow up phone calls that some questionnaires had been passed on to other LMCs and the original contact had been lost. Although it was nearly always possible to contact the person questionnaires had been passed to, there is only so much interruption that can be made to busy practitioners without the research involvement potentially becoming a nuisance. In addition, in health research, an incentive for practitioners which may have increased a feeling of reciprocity was not possible due to budget and usually not considered ethical.

\subsubsection{Calculation of response}

There were 489 questionnaires returned by post over the data collection period (included in this calculation are the booster sampling questionnaires returned). Of these, 8 were deemed to most likely be multiparous women by nature of some responses. These were therefore discarded as not eligible. For respondents in the inclusion criteria, a small number $(n=11)$ had fewer than five missing items (due to errors in line matching or item oversight) and were retained. Three questionnaires were considered unusable as they had over one page uncompleted. This usually occurred where a page had been turned and missed.

In order to calculate LMC distribution, a log was kept of the date and number of questionnaires sent to each LMC. Toward the end of the data collection period, LMCs were contacted and asked how many had been given out. There was some difficulty estimating exact numbers. Although many providers had kept accurate records, a large number of questionnaires had been handed on to other practitioners and in approximately seven cases, the midwife who originally accepted the questionnaires had left or was imminently leaving practice. Although LMCs were happy to hand questionnaires on to other providers, it was often difficult to make contact with the nominated provider. One telephone conversation made the researcher aware that some were disposed of accidently, despite regular contact from the researcher.

On receipt of returned questionnaires, achievement of adequate representation by city/town size, age and ethnicity was estimated and compared with statistical 
reports in 'Report on Maternity' (Ministry of Health, 2004) and the Statistics NZ website. It became apparent that the number returned from Māori was borderline and from Pacific women, lower than desired. This resulted in a second phase of data collection being undertaken during over a further three months, when targeted booster sampling was undertaken.

Pacific and Māori midwives consulted had no specific explanations for the poor return from Pacific woman, except perhaps language problems; for some Pacific women, their ability to understand such a lengthy questionnaire (based on language) was marginal. Other ways of distribution that may have been of benefit (for example, through church communities) were not able to be undertaken due to resourcing.

During booster sampling to target Pacific women, 40 further questionnaires were released into the field. Questionnaires were delivered to three midwifery practices in the Wellington region that communicate service offerings specifically to Māori and Pacific women. This collection period was kept open for three months, resulting in 10 questionnaires returned (4 Māori and 6 Pacific). Due to time constraints the data collection was closed before all questionnaires could be given out.

A best estimation was made after discussion with all participating LMCs. At the cut off point there were an estimated $10 \%$ of questionnaires either still with providers or unaccounted for. LMCs were asked to return any questionnaires they still had and approximately $50 \%$ of these were returned. It was not easy to estimate which regions had been better canvassed by LMCs. Table 4.7 provides a breakdown of distribution and response.

Table 4.8. Distribution by LMC Type and region

\begin{tabular}{llll}
\hline & Number & \\
\hline By LMC Type & & By region**** & \\
Midwife & 752 & Wellington and Hutt Valley & 639 \\
Midwife (homebirth) & 29 & Kapiti Coast & 86 \\
DHB (primary and secondary & 381 & Wairarapa & 74 \\
clinics) & & & \\
GP/specialist/medical practice & 28 & Fielding/Palmerston North & 95 \\
Antenatal classes & 149 & Whanganui & 50 \\
Subtotal & 1339 & Auckland & 225 \\
Less $10 \%$ loss & 1205 & Whangarei & 50 \\
$*$ Returned from respondents & & Christchurch & 98 \\
\hline
\end{tabular}




\begin{tabular}{|c|c|c|c|}
\hline $\begin{array}{l}* * \text { Targeted booster sampling; } \\
20\end{array}$ & 1225 & Westcoast/Greymouth & 30 \\
\hline Total returned & & Otago & 12 \\
\hline
\end{tabular}

*The total number of questionnaires distributed was 1339. Ten percent attrition (non-distributed questionnaires) has been calculated and thought to be conservative. This leaves a subtotal of 1205 distributed questionnaires. 468 were returned, for a response rate from women of $39.9 \%$.

**Another 40 questionnaires were distributed to LMCs during booster sampling. Ten questionnaires were returned from respondents. The data collection period was cut off. Twenty of these questionnaires had been distributed by practitioners.

$* * * 11$ returned questionnaires were unusable, so useable responses were $478(38.8 \%)$.

$* * * *$ Of the total questionnaires (primary data collection phase and targeted sampling, 210 questionnaires) had been distributed to Māori /Pacific midwifery practices or areas where there was known Māori /Pacific ethnic density. Initial target sampling and second purposeful target sampling occurred in Wellington Central, Porirua Basin and Waitemata (Auckland).

$* * * *$ Does not include $10 \%$ attrition adjustment. Included is 20 questionnaires distributed during booster sampling. $* *$

The overall summary response rate from women who received the questionnaires cannot be estimated with absolute accuracy. However, conservatively it would be seem at least $40 \%$ and could certainly have reached as much as $50 \%$.

\subsubsection{DATA ANALYSIS}

This next section provides a broad overview of the statistical approach, the various tests and procedures undertaken to analyse data during the quantitative phase.

\subsubsection{Factor analysis}

A robust procedure was used in the initial scale building phase and was carried through into the analysis. The testing of scales was undertaken with specific criteria applied during factor analysis and reliability testing. This included meeting communality thresholds, factor loadings and minimum explained variance.

Confirmatory factor analysis was used to validate scales that were already in existence and had been drawn from the literature. Some existing scales required modification for the present study during the design phase. This was to make them either more relevant to the societal shifts in values that had occurred since their initial development, or more culturally relevant to the context and place of data collection. The scale 'Familism' was for example modified in order to be relevant to the sample. Other scales such as Women's Role (Tam \& Tai, 1998) 
that had been modified substantially from the original source ended up being discarded entirely as a scale during both stages of CFA and EFA, due to failure to meet the required criteria. The individual items were retained, however, because of the particular insights they added to the analysis.

In addition, the religiosity scale was reduced from a four item scale to a two item scale. Whilst all scales except the HLOC scale met the factor loading criteria, this was sometimes achieved by the removal of some items. Differences of opinion exists between what is regarded acceptable for confirmatory factor analysis but in general, minimum factor component loadings of 0.70 or higher are normally considered significant (Hair, Black, Babin, Andersen, \& Tatham, 2006; Nunnally \& Bernstein, 1994).

A large number and mix of variables resulted in a significant level of scale building and testing. As this study was exploratory rather than confirmatory of hypothesis testing, many of the scales could only be explored rather than tested.

There were no predetermined or fixed numbers of factors during this stage (Hair et al., 2006). Decisions to include or discard items from scales were based on factor loadings being at least 0.6 or above on a single factor and less than 0.45 for cross loading on any other factor where the scale was expected to have multidimensional characteristics. Commonalties had to be above 0.5 (Hair et al., 2010). The minimum explained variance was $50 \%$ or above, and eigenvalues had to exceed one for all factors. Varimax rotation was used to reduce complexity of the loadings and improve interpretability (Hair et al., 2010).

Where any item did not reach a specified criterion, it was usually discarded. In some instances, further investigation was carried out to evaluate whether its removal also adversely affected Cronbach's alpha. If this was the case, the item was usually retained. For items omitted during the factor analysis, those thought to be providing some distinctive insight were retained as single items. Satisfaction of all of these criteria led to a scale and variable set that was considered very satisfactory and robust.

\subsubsection{Descriptive analysis and bi-variate analyses}

The descriptive phase of the analysis included means, medians and standard deviations. The literature has pointed to relationships between variables that had been included in the study, based on findings from previous research, in health in 
particular and to a lesser extent, pregnancy specific. In order to understand the data better and highlight associations, cross tabulations: t-tests (testing variable difference between two groups); analysis of variance (ANOVA); testing of significant mean differences in variables among multiple groups and Pearson's correlations; testing of the level of association between two variables were performed (Cavana et al., 2000). As the approximation for chi-square distribution is may not be accurate for small samples in cross-tabulation (Field, 2009) where an expected cell frequency was less than 5, a Fisher's Exact Test (Fisher, 1922) was used, as this provides a method for an exact chi-square statistic when cells numbers are small (Field, 2009).

\subsubsection{Cluster analysis and profiling}

Cluster analysis is an umbrella term used to describe a variety of techniques, similar to exploratory factor analysis but that identifies groupings based on closeness or proximity between respondents in a dataset, rather than correlations (Berry, 2008). Cluster analysis is an appropriate technique used in psychographic studies (Gondález \& Bello, 2002). This study utilised a two-step cluster analysis, using Schwart's Baysian Criteria, as it is a type of clustering technique that can accommodate mixed continuous, ordinal and nominal variables and large data sets with proximities calculated using log-likelihood distances (Berry 2008).

\subsubsection{4.i. Stages of cluster procedure}

The five stages of the clustering procedure comprised of: initial identification of variables for sub-clustering, formation of sub-clusters, selection of variables for the main Gravidographic clustering, formation of Gravidographic clusters and finally, profiling of these. Figure 3.1 provides an overview of the different stages. An explanation of the stages follows. 
Figure 4.1. Clustering procedure leading to identification of Gravidographic segments

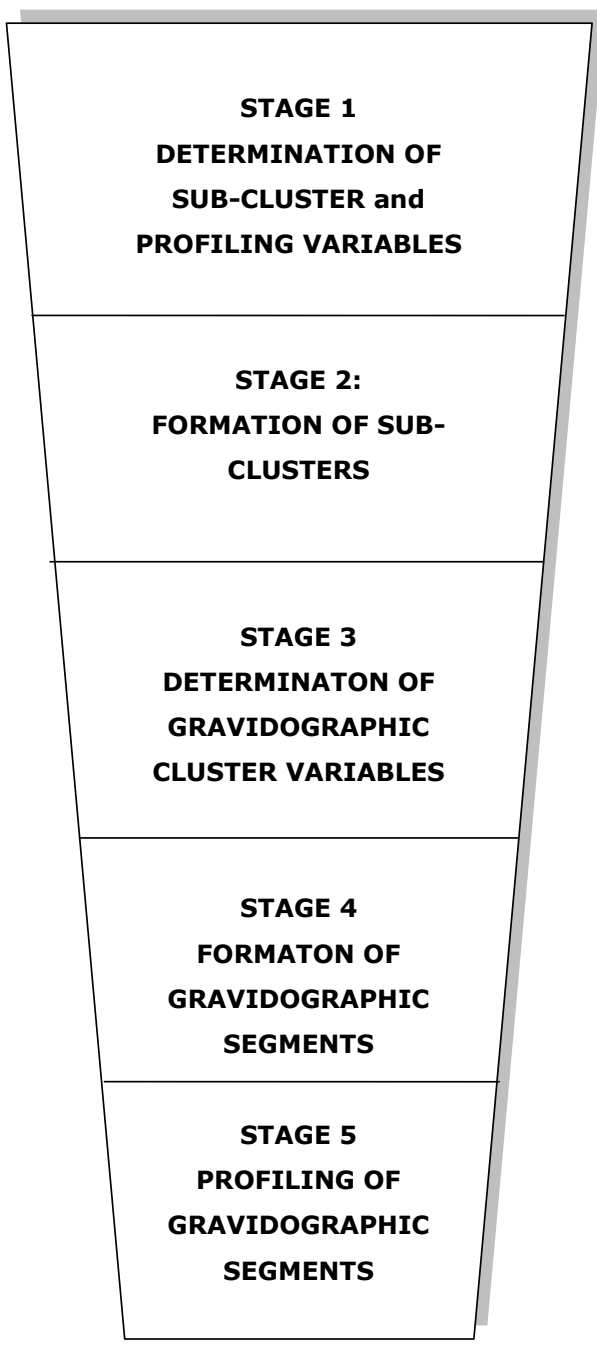

Most salient and/or determinant variables selected from constructs and attributes.

These divided into subclustering variables and those to be held back for profiling.

Assignment of variables and formation of lifestyle subclusters from selected variables. Choice of subclusters was determined by sub-domains of the conceptual framework

Most salient and/or

determinant variables for Gravidographic clustering phase selected from subclusters.

Variables selected in Stage 3 put forward to a final clustering procedure in order to determine Gravidographic segments.

Bi-variate analyses of demographic and other variables held back for profiling. This stage was profiling. This stage was
undertaken to determine what characteristics relate to characteristics relate to
membership of a particula lifestyle group.

\subsubsection{3.i. Sub-clustering phase}

\section{Determination of sub-cluster variables}

Variables from the sub-domains were selected according to their saliency and those that best addressed the research questions pertaining to identification of homogenous groups. Saliency of a variable was based on variability of response in the descriptive stages of analysis, and/or that it was deemed by the researcher to be an important measure of/or influence over lifestyle.

A key element of the five stage procedure involved sub-clustering on specific or focused areas of health behaviour prior to a main cluster procedure. Collectively the sub-clusters measured a comprehensive range of health attitudes and behaviours. This struck a balance between insights and richness available from 
the data without being overwhelmed by its volume. Formation of sub-clusters was therefore used in part as a further data reduction procedure following CFA and EFA. These sub-clusters also allowed for more focused and detailed insights into the main sub-domains of exploration. All sub-clusters were used to guide the determination of variables for inclusion in the Gravidographic clustering.

\section{Determining number within a sub-cluster}

Initially, cluster number was set at 'spontaneous' cluster setting. As the optimal number for any of the clusters was not known initially, and with the intention of choosing the appropriate number objectively, several iterations were testedranging from two to eight groupings. Many clusters fell spontaneously into two groupings only and this was not regarded as useful. Determination of the most appropriate cluster number was based on comparing each to the other. Three has been suggested as a suitable cluster number choice as it gives high, medium and low groupings of attitudes and actions (Berry, 2008). Final choice was based on judgement: meaningful differences between groups and parsimony (Berry 2008). Secondly, statistical criteria were used. These included Schwarz Bayesian Criterion, where agglomeration schedules jump sharply when too many clusters are created (Berry, 2008). Sub-cluster and Gravidographic cluster stability was checked by reordering the data randomly, therefore starting at a different seed point but specifying the same number of clusters (Hair, 2010).

\subsubsection{3.ii. Gravidographic clustering phase}

This included Stage 4 and 5 of the clustering procedure and led to the formation of the final clusters and identification of Gravidographic segments. The same criteria for cluster identification was followed as for the sub-clustering phase.

\section{Gravidographic Cluster Profiling}

The final phase of the clustering procedure was to profile each of the identified Graviodgraphic lifestyle clusters. The aim of profiling was to characterise each cluster in terms of demographics, pre-existing influences and other attributes. Profiling provided further insight into the characteristics of the various clusters and who populates them. For this, bi-variate tests of association ANOVA and cross-tabulations were conducted between clusters and variables that had been reserved for profiling. For example, demographic data was held back from clustering, so that cluster membership was not driven by demographic characteristics (Kolodinsky \& Reynolds, 2009). Likewise, variables from 'pre- 
existing values', variables that describe respondent's personality traits and values were also used for profiling.

\subsection{CHAPTER 4 SUMMARY}

The survey consisted of a health professional distributed survey to pregnant nulliparous women in their care, with a postal reply response by respondents. The quantitative stage was executed first and an analysis commenced before the qualitative phase was commenced. The questionnaire, generated to explore the study conceptual framework, was constructed as a psychographic type survey, and therefore had a large battery of scales and items. AIO type, pregnancy circumstance, direct health behaviour and demographic questions were presented to the respondents divided into sections to enable easier progression through the questionnaire. The analysis determined for this data was exploratory factor analysis for data reduction and scale verification, followed by descriptive analysis, exploration of relationships between variables of interest and finally, a two-step cluster analysis. 


\section{CHAPTER 5. DATA ANALYSIS AND RESULTS}

\subsection{INTRODUCTION}

The purpose of this chapter is to describe the data analysis and results. The quantitative results include an overview description of the respondent group, bivariate analyses and the two-stage cluster analysis. The quantitative instrument for this survey included existing, modified and original scales. Initial attention therefore focused on the performance of scales and items, refining of the data set, and data reduction procedures. The final stage of analysis was the use of cluster analysis as the basis of pregnancy health lifestyle segmentation and associated profiling.

\subsection{QUANTITATIVE RESULTS}

\subsubsection{DATA ENTRY AND CLEANING}

A coding schedule was designed for the questionnaire prior to commencement of data entry. SPSS Version 16 was used to input and code data and then to conduct subsequent quantitative analysis. All data were coded using numeric codes where possible. Open ended responses were recorded on a 'two pass' basis under main groupings of categories. Any data that were 'out of range' were identified and resolved.

\subsubsection{QUESTIONNAIRE VALIDATION AND EXCLUDED QUESTIONNAIRES}

Eight questionnaires were excluded as it was suspected these had been completed by multiparous women, who were not in the target sample. A further three questionnaires were discarded as there were more than two pages of response missed (this appeared to be most often due to missing a page during turning). Some women were identifiable as having twins. This was not considered to be a ground for exclusion as their health was still the focus of study. This led to a total of 478 useable questionnaires (see also Chapter 4 for a discussion of response rates) and a sample with a gestation spread over 20 to 38 weeks. 


\subsubsection{TREATMENT OF EXTREME AND MISSING VALUES}

A missing value analysis (MVA) was carried out in SPSS. Overall, by variable, the range was between 0-4 missing values, with all but 10 variables in the first half of the questionnaire having zero. Employment and income variables had 6 and 9 respectively missing. This may well have been a resistance to answering the question.

Where the MVA count for values was between 6 and 12 ( $n=22,4.5 \%$ of total values), these appeared to be questions with several items related to the question. Upon examination of the database and the questionnaires for these, it was due to respondent's missing complete lines. MVA analysis by case showed that 29 cases had missing values ranging from 1 to 3 . Three cases had 12,22 and 35 missing values respectively. These questionnaires were checked and it was found for the 22 and 35 missing values, a page has been missed. These questionnaires were not withdrawn from the analysis as a large proportion of the questionnaire had been answered well.

Missing values were not given any specific treatment, due to their very small instance overall. There was also no identifiable pattern of missing values attributable to one specific demographic. Outliers were also not given any specific treatment, given the preponderance of Likert scales which avoid the problem of extreme outliers (Hair, 2010).

\subsubsection{TESTS FOR NORMALITY}

Determining normality of the data was undertaken by calculating the skewness and kurtosis of all items. Coakes and Steed (2007) suggest that values for skewness and kurtosis are zero, if the observed distribution is exactly normal. However, acceptable normality for skewness falls between +1 and -1 and +2 and -2 for kurtosis. Analysis showed that 35\% of values show a degree of skewness (ranging from +1 to +2 and -1 to-2) and corresponding kurtosis. For 10 of the values which included items regarding smoking, alcohol and behaviours that would ensure the safety of the baby, skewness was as high as +3 .

As a means of managing skewness and kurtosis, transforming the data was considered. Sharma, Durvasula \& Dillon (1989) and Yanagihara \& Yuan (2005) found that the use of non-transformed data in parametric tests such as t-tests and ANOVA was not detrimental to robustness; therefore, non-transformed data was used in analysis. As a guard against missing any potential problems, 
correlations using both Pearson's and Spearman's tests were performed on all the main scales used. Almost invariably, both led to similar results. As a result, no specific treatment for non-normality of data was undertaken for the main analysis.

\subsubsection{SAMPLE SIZE AND POWER}

Increasing sample size generally reduces sampling error and increases the sensitivity (power) of the test with noticeable effects evidenced to $n=150$ (Hair, Black, Babin, Andersen, \& Tatham, 2010). Based on this criteria, the sample size $(n=478)$ is considered sufficient to perform the statistical procedures undertaken with confidence in the robustness of results.

\subsubsection{FACTOR ANALYSIS AND SCALE VALIDATION}

Initial analysis involved factor analysis for scale testing and building. The testing of scales was undertaken with specific criteria applied during factor analysis and reliability testing. This included meeting communality thresholds, factor loadings and minimum explained variance as detailed in Chapter 4.

Scales that were existing and considered to not have been significantly modified were tested using Confirmatory Factor Analysis procedures. Scales that were treated as "new" (either because they had been substantially modified in wording or form for the context of this study or that were formed during data reduction) were tested with Exploratory Factor Analysis procedures.

\subsubsection{CRONBACH'S ALPHAS}

Scale reliability was tested using Cronbach's alpha. Values between 0.6 and 0.7 are deemed to have the minimal level of acceptability (Hair et al., 2010). Almost all scales retained after factor analysis met this criteria. Five scales had alphas slightly less than 0.7 (three of 10 existing scales and two of the 13 new scales). In regard to the existing scales, as they had been used apparently successful in other previous studies, they were retained despite their low alphas. New scales had no Cronbach's alpha values below 0.6, which is considered acceptable in EFA.

\subsubsection{PRESENTATION OF RESULTS}

Descriptive findings are presented in the sequential order of the questionnaire, which in the main, follows the conceptual framework ordering. Any deviation from this is for reader clarity and conceptual flow. Within tables, italicised entries denote scales. For some cross-tabulations, 'lower cell counts than expected' were 
found but no further aggregation was undertaken as it was believed to be more insightful to keep the groupings as they were. Where expected cell counts were less than five, the Fisher's Exact test was reported. The Fishers Exact test is a way of computing the exact probability of a statistic, designed to overcome the problem of a small sample (Field, 2009).

The sample size for all statistical tests was 478, unless otherwise stated. Likert scales were 7 point, except for the Kahle's List of Values, where item reporting has been kept to the author's original 9 point scale. For all scales and items, the higher the score, the higher the strength of agreement or importance of the statement for items and scales.

\subsubsection{SAMPLE REPRESENTATIVENESS}

The respondent sample was compared against statistics available in Report on Maternity (Ministry of Health, 2004), and The New Zealand Statistics website (NZ Govt) and is believed to satisfactorily represent the New Zealand child bearing population. There is a slight skew toward higher educational attainment as demonstrated by a relatively higher level of Bachelor degree or above than the New Zealand population norm. The response rate from Pacific women is slightly below comparative rates for New Zealand population rate.

\subsection{FACTOR ANALYSIS}

For both Confirmatory Factor Analysis (CFA) and Exploratory factor Analysis (EFA), The Kaiser-Meyer-Olkin measure of sampling adequacy shows values greater than 0.6 for all constructs, therefore, the factorability of the correlation matrix is proven (Hair et al., 2010). Eigenvalues for extraction were set at 1 . Varimax, an orthogonal rotation technique (Hair et al., 2010) was carried out to improve interpretability of the factor matrix. Varimax rotation is the definitive orthogonal rotation recommended by Nunally and Bernstein (1994). It is acknowledged that Pett et al (2003) suggest oblique rotation for health scales, as these authors state that in health, sub scales of interest are rarely uncorrelated from one another. Analysis of three of the scales (Health Consumerism, Prepregnancy Health Level and Environmentalism) was carried out as a caution using both orthogonal and oblique (Promax) techniques. When compared, there was no essential difference in the factors seen that would lead the researcher to be concerned regarding the robustness of the Varimax solution. Therefore, the Varimax solution was the rotation technique used. 
In Chapter 5, scale items are reported by their 'working title'

\subsubsection{Confirmatory Factor Analysis (CFA) of existing scales}

Confirmatory Factor Analysis was used to explore and validate scales that were already in existence and had been drawn from the literature. Most scales performed well against the criteria, despite modifications, in many cases, to fit the context of this study. For instance, the Religiosity scale (Schieman, Nguyen, \& Elliott, 2003) was reduced from four items to two items. Whilst all scales except the Familism and Health Locus of Control sub-scales met the factor loading criteria and Cronbach's alpha reliability, this was often achieved by the removal of items from the original scales. Differences of opinion exist between what is regarded acceptable for CFA but in general, minimum factor component loadings of 0.70 or higher are normally considered acceptable (Hair et al., 2006; Nunnally \& Bernstein, 1994).

Table 5.1. Pre-pregnancy health level scale

\begin{tabular}{lllll}
\hline Item (working title) & Loading & Com* & a & AVE\# \\
\hline General lifestyle & .866 & .750 & .933 & $63.00 \%$ \\
Personal confidence in keeping & .860 & .740 & & \\
Attitude to good health & .848 & .720 & \\
Healthy eating habits & .842 & .708 & \\
Level of fitness & .799 & .638 & \\
Physical health & .797 & .635 & \\
Overall health & .784 & .615 & \\
Time put in & .784 & .614 & \\
Knowledge of health & .682 & .465 & \\
Emotional well-being & .643 & .414 & \\
\hline
\end{tabular}

Note. ${ }^{*}$ Com $=$ Communality; ${ }^{*}$ AVE = Average Variance Explained, a = Cronbach's alpha

Table 5.2. Health consumerism scale

\begin{tabular}{lllll}
\hline Item (working title) & Loading & Com* & a & AVE\# \\
\hline Requests info & .717 & .514 & .688 & $61.72 \%$ \\
Challenges professional & .824 & .678 & & \\
Willing to complain & .812 & .660 & & \\
\hline
\end{tabular}

Note. ${ }^{*}$ Com $=$ Communality; ${ }^{\#}$ AVE = Average Variance Explained, $a=$ Cronbach's alpha

Table 5.3. Environmentalism scale

\begin{tabular}{lllll}
\hline Item (working title) & Loading & Com* & a & AVE\# \\
\hline Environmental issues important & .827 & .683 & .785 & $69.97 \%$ \\
Buy environmentally friendly products & .879 & .772 & & \\
Would not use damaging products & .803 & .644 & & \\
\hline
\end{tabular}

Note. ${ }^{*}$ Com $=$ Communality; ${ }^{\#}$ AVE = Average Variance Explained, $a=$ Cronbach's alpha 
Table 5.4. Concern for physical appearance scale

\begin{tabular}{lllll}
\hline Item (working title) & Loading & Com* & a & AVE\# \\
\hline Looks extremely important & .878 & .770 & .847 & $76.54 \%$ \\
Embarrassed if did not look my best & .870 & .756 & & \\
Looking best is worth the effort & .877 & .770 & & \\
\hline Note. * Com = Communality: ${ }^{*}$ AVE = Average Variance Explained $a=$ Cronbach's alpha
\end{tabular}

Note. ${ }^{*}$ Com $=$ Communality; ${ }^{\#}$ AVE = Average Variance Explained, $a=$ Cronbach's alpha

Table 5.5. Religiosity scale

\begin{tabular}{lllll}
\hline Item (working title) & Loading & Com* & a & AVE\# \\
\hline Am religious person & .967 & .935 & .930 & $93.47 \%$ \\
Beliefs influence how I live & .967 & .935 & & \\
\hline
\end{tabular}

Note. ${ }^{*}$ Com $=$ Communality $;{ }^{*}$ AVE $=$ Average Variance Explained, $a=$ Cronbach's alpha

Table 5.6. Trait anxiety scale

\begin{tabular}{lllll}
\hline Item (working title) & Loading & Com* & a & AVE\# \\
\hline Anxious person & .864 & .747 & .799 & $71.35 \%$ \\
Nervous and restless person & .844 & .713 & & \\
Wish didn't worry as much & .825 & .680 & & \\
\hline
\end{tabular}

Note. ${ }^{*}$ Com $=$ Communality; ${ }^{*}$ AVE = Average Variance Explained, a = Cronbach's alpha

The dropping of items for Trait Anxiety during factor analysis to rid excessive cross-loadings led to a loss of original multi-dimensionality and retention of a unidimensional measure.

Table 5.7. Sense of mastery scale

\begin{tabular}{lllll}
\hline Item (working title) & Loading & Com* & a & AVE\# \\
\hline Feel helpless (rev) & .725 & .525 & .799 & $62.59 \%$ \\
No way to solve problems (rev) & .842 & .709 & & \\
Little can do to change (rev) & .833 & .694 & & \\
Little control over things (rev) & .758 & .575 & \\
\hline Note. * Com = Communality; ${ }^{*}$ AVE = Average Variance Explained, a = Cronbach's alpha
\end{tabular}

Table 5.8. Health Locus of Control (HLOC): Internal scale

\begin{tabular}{lllll}
\hline Item (working title) & Loading & Com* & a & AVE\# \\
\hline Health in preg depends on me & .731 & .535 & .634 & $47.72 \%$ \\
I am directly responsible for health in preg & .696 & .485 & & \\
If look after health in preg will go well & .694 & .482 & & \\
Healthy preg needs effort & .638 & .407 & & \\
\hline
\end{tabular}

Note. ${ }^{*}$ Com $=$ Communality; ${ }^{\#}$ AVE = Average Variance Explained, $a=$ Cronbach's alpha

This subscale did not meet CFA criteria for factor loadings, communalities, or Cronbach's alpha, despite the removal of items that were cross loading. This 
scale was retained, however because of its conceptual importance in health studies.

Table 5.9. Health Locus of Control (HLOC): Fate scale

\begin{tabular}{lllll}
\hline Item (working title) & Loading & Com* & a & AVE\# \\
\hline If meant to be will stay healthy in pregnancy & .637 & .406 & .682 & $51.37 \%$ \\
Fate determines how it goes & .790 & .624 & & \\
No matter what I do will happen & .729 & .531 & & \\
If stay well am lucky & .702 & .492 & \\
\hline
\end{tabular}

Note. ${ }^{*}$ Com $=$ Communality; ${ }^{\#}$ AVE $=$ Average Variance Explained, a = Cronbach's alpha

Table 5.10. Health Locus of Control (HLOC): Powerful Others scale

\begin{tabular}{lllll}
\hline Item (working title) & Loading & Com* & a & AVE\# \\
\hline Only Dr or MW can prevent & .853 & .728 & .531 & $67.88 \%$ \\
Must consult Dr or MW & .784 & .663 & & \\
Dr or MW should make decisions & .826 & .682 & & \\
If excellent care will not get sick & .783 & .642 & & \\
\hline
\end{tabular}

Note. ${ }^{*}$ Com $=$ Communality; ${ }^{\#}$ AVE $=$ Average Variance Explained

This sub-scale of the HLOC did not meet the minimum of 0.7 for Cronbach's alpha. Items were retained individually.

Table 5.11. Familism scale

\begin{tabular}{|c|c|c|c|c|}
\hline Item (working title) & Loading & Com* & $\mathbf{a}$ & AVE\# \\
\hline Factor A ( Sorting Problems) & & & .512 & \\
\hline Help with financial problems & .849 & .722 & & \\
\hline Help with problems & .782 & .651 & & $35.52 \%$ \\
\hline \multicolumn{5}{|l|}{ Factor B (Family ties) } \\
\hline Involve grandparents & .858 & .737 & & \\
\hline Financial support to younger siblings & .802 & .680 & & $34.26 \%$ \\
\hline Total variance & & & & $69.78 \%$ \\
\hline
\end{tabular}

Note. ${ }^{*}$ Com $=$ Communality; ${ }^{*}$ AVE = Average Variance Explained, $a=$ Cronbach's alpha

Factor analysis did not confirm this scale in regard to specified criteria, despite several iterations. The iteration shown, whilst managing to rid the factors of cross loadings, reduced the Cronbach's alpha reliability to below the minimum required of 0.7 . This scale was retained, but results need to be viewed with caution. 
Table 5.12. Maternal-fetal attachment scale

\begin{tabular}{lrrrl}
\hline Item (working title) & Loading & Com* & a & AVE\# \\
\hline Excited about motherhood & 0.831 & 0.690 & .843 & $61.60 \%$ \\
Looks forward to each new stage & 0.824 & 0.679 & & \\
Feels attached to baby & 0.757 & 0.573 & & \\
Body achieving something extraordinary & 0.755 & 0.570 & & \\
Enjoys feeling baby move & 0.753 & 0.567 & \\
\hline
\end{tabular}

Note. ${ }^{*}$ Com $=$ Communality; ${ }^{*}$ AVE = Average Variance Explained, $a=$ Cronbach's alpha

\subsection{Exploratory Factor Analysis (EFA) of new scales}

The following scales were considered new scales, so therefore analysed using the criteria for EFA. This procedure was also undertaken as a data reduction process, whereby items that could be grouped conceptually were explored, an approach advocated by Nunnally and Bernstein (1994). For EFA, loadings of 0.60 or more are considered acceptable (Hair et al., 2010).

Table 5.13. Information receipt interest scale

\begin{tabular}{lllll}
\hline Item (working title) & Loading & Com* & a & AVE\# \\
\hline Actively seek pregnancy information & .894 & .798 & .747 & $79.83 \%$ \\
Interested in receiving pregnancy information & .894 & .798 & & \\
\hline
\end{tabular}

Note. ${ }^{*}$ Com $=$ Communality; ${ }^{*}$ AVE = Average Variance Explained, $a=$ Cronbach's alpha

Table 5.14. Information outcome beliefs scale

\begin{tabular}{lllll}
\hline Item (working title) & Loading & Com* & a & AVE\# \\
\hline Feel better equipped to make choices & .788 & .620 & .766 & $58.79 \%$ \\
More confident and less anxious & .786 & .618 & & \\
Knowing what to expect helps adjust & .778 & .605 & & \\
Makes more confident about choices & .713 & .508 & \\
\hline Note. ${ }^{*}$ Com = Communality; ${ }^{*}$ AVE = Average Variance Explained, a = Cronbach's alpha
\end{tabular}

Table 5.15. Information change result scale

\begin{tabular}{lllll}
\hline Item (working title) & Loading & Com* & a & AVE\# \\
\hline Changed attitudes where needed & .880 & .774 & .708 & $77.39 \%$ \\
Changes practices where needed & .880 & .774 & & \\
\hline Note * Com $=$ Communality * AVE ${ }^{*}$ Average Variance Explained $a=$ Cronbach's alpha &
\end{tabular}

Note. ${ }^{*}$ Com $=$ Communality; ${ }^{*}$ AVE $=$ Average Variance Explained, a = Cronbach's alpha

Table 5.16. Pregnancy health effort and motivation scale

\begin{tabular}{lllll}
\hline Item (working title) & Loading & Com* & a & AVE\# \\
\hline Important to care for health for baby's sake & .826 & .826 & .775 & $59.94 \%$ \\
Important to care for health for own sake & .823 & .823 & & \\
Have put a lot of effort into pregnancy health & .733 & .537 & & \\
Will do whatever it takes & .708 & .708 & \\
\hline Note. ${ }^{*}$ Com = Communality; ${ }^{\#}$ AVE = Average Variance Explained, a = Cronbach's alpha
\end{tabular}


Table 5.17. Self-efficacy for making health changes scale

\begin{tabular}{lllll}
\hline Item (working title) & Loading & Com* & a & AVE\# \\
\hline Too hard to make changes (rev) & .795 & .632 & .641 & $58.24 \%$ \\
Confident can look after self well & .748 & .56 & & \\
Unsure how to look after health (rev) & .746 & .556 & & \\
\hline
\end{tabular}

Note. ${ }^{*}$ Com $=$ Communality; ${ }^{\#}$ AVE = Average Variance Explained, $a=$ Cronbach's alpha

Table 5.18. Good nutrition effort scale

\begin{tabular}{|c|c|c|c|c|}
\hline Item (working title) & Loading & Com* & & a AVE\# \\
\hline Eats well-balanced diet & .850 & .723 & .828 & $66.0 \%$ \\
\hline Takes care to eat healthy diet & .840 & .706 & & \\
\hline Adheres to dietary guidelines & .778 & .606 & & \\
\hline Avoids junk food & .778 & .606 & & \\
\hline
\end{tabular}

Note. ${ }^{*}$ Com $=$ Communality; ${ }^{\#}$ AVE = Average Variance Explained, $a=$ Cronbach's alpha

Table 5.19 Activity interference scale

\begin{tabular}{lllll}
\hline Item (working title) & Loading & Com* & a & AVE\# \\
\hline Pregnancy doesn't interfere with physical ability & .897 & .805 & .757 & $80.47 \%$ \\
Just as active & .897 & .805 & &
\end{tabular}

Note. ${ }^{*}$ Com $=$ Communality; ${ }^{*}$ AVE $=$ Average Variance Explained

Table 5.20 Pregnancy state anxiety scale

\begin{tabular}{lllll}
\hline Item (working title) & Loading & Com* & a & AVE\# \\
\hline Labour and birth not go well & .816 & .666 & .727 & $55.13 \%$ \\
Ability to handle labour and birth & .763 & .582 & & \\
Health problems in pregnancy & .693 & .481 & & \\
Worry about how baby doing & .690 & .476 & \\
\hline Note. ${ }^{*}$ Com =Communality; ${ }^{\#}$ AVE = Average Variance Explained, a = Cronbach's alpha
\end{tabular}

Table 5.21 Life situation state anxiety scale

\begin{tabular}{lllll}
\hline Item (working title) & Loading & Com* & a & AVE\# \\
\hline Changing family relationships & .778 & .605 & .622 & $57.05 \%$ \\
Other stressful life events & .778 & .620 & & \\
Worried about future of career & .698 & .487 & & \\
\hline
\end{tabular}

Note. ${ }^{*}$ Com $=$ Communality; ${ }^{*}$ AVE = Average Variance Explained, $a=$ Cronbach's alpha

Table 5.22. Weight concerns scale

\begin{tabular}{lllll}
\hline Item (working title) & Loading & Com* & a & AVE\# \\
\hline Worry might look fat & .839 & .705 & .747 & $57.09 \%$ \\
Embarrassed whenever weighed & .740 & .548 & & \\
Feel body out of control & .718 & .516 & & \\
Anxious to get back into shape & .718 & .515 & & \\
\hline
\end{tabular}

Note. ${ }^{*}$ Com $=$ Communality; ${ }^{\#}$ AVE = Average Variance Explained, $a=$ Cronbach's alpha 
Table 5.23. Pregnancy body satisfaction scale

\begin{tabular}{lllll}
\hline Item (working title) & Loading & Com* & a & AVE\# \\
\hline Proud to look pregnant & .907 & .823 & .819 & $74.96 \%$ \\
Can look good & .859 & .737 & & \\
Happy about changes & .814 & .662 & & \\
\hline Note. ${ }^{*}$ Com = Communality; ${ }^{*}$ AVE = Average Variance Explained, a = Cronbach's alpha
\end{tabular}

Table 5.24 Celebrity attraction scale

\begin{tabular}{lllll}
\hline Item (working title) & Loading & Com* & a & AVE\# \\
\hline Like seeing or reading about celebs & .726 & .726 & .708 & $63.27 \%$ \\
Likes to copy pregnant celebs & .605 & .778 & & \\
Celebs losing weight after pregnancy an inspiration & .567 & .753 & & \\
\hline Note. * Com = Communality; ${ }^{*}$ AVE = Average Variance Explained, a = Cronbach's alpha
\end{tabular}

Table 5.25. Perception of wellness scale

\begin{tabular}{lllll}
\hline Item (working title) & Loading & Com* & a & AVE\# \\
\hline Pretty good & .831 & .690 & .685 & $61.44 \%$ \\
Unhappy (rev) & .780 & .545 & & \\
Relaxed & .780 & .608 & & \\
\hline
\end{tabular}

Note. ${ }^{*}$ Com $=$ Communality; ${ }^{\#}$ AVE $=$ Average Variance Explained, $a=$ Cronbach's alpha

\subsubsection{PERFORMANCE OF SCALES}

Sub-scale results for the existing scale 'Health Locus of Control', (Wallston, Wallston, \& DeVellis, 1978) are marginally acceptable. The established Health Locus of Control scale (HLOC) (Wallston et al., 1978) demonstrated loadings lower than had been found in prior studies (non-pregnancy), with unacceptably high levels of cross loadings between the sub-scales. The Internal HLOC subscale therefore has low acceptability in respect to criteria, but is retained as it is used in pregnancy health measurement (see for instance, Haslam, Lawrence, \& Haefeli, 2003; Kruse, Zweig, \& LeFevre, 1988). The Powerful Others HLOC subscale proved to be unacceptable but was retained as a set of items as it is regarded to be of conceptual importance. The scale has been cautiously modified to be context specific, but its reliability needs to be viewed with caution.

'Familism' (Steidel \& Contreras, 2003), again modified for the study context, could not be confirmed during factor analysis, suggesting perhaps a lack of fit for the context. This may be because the original conceptualisation for this scale was considered a construct related to the Hispanic culture (Steidel et al., 2003) and developed to reflect the core cultural values of Hispanic families (Villarreal \& Peterson, 2009). Additionally, there is a possibility that the scale may not be 
adequately reflecting the shifts in societal patterns and values affecting contemporary families that have occurred in recent times.

\subsection{DESCRIPTIVE RESULTS AND BI-VARIATE ANALYSES}

Descriptive results are presented in this section, following the order of the conceptual framework. At the beginning of each sub-section, a graphic of the framework is provided to refresh the reader of the sub-domain to which variables being presented fit within the framework.

Cross tabulations, Pearson's correlations and ANOVAs of particular interest are shown in the main body of the thesis, rather than the appendices, for readability and flow. Given the large data set, this also enables understanding of the results of the sample description and relationships as they evolve, rather than presenting large numbers of tables at the end of the chapter text. In addition, as there are many correlations that can potentially be tested within such a large data set, a degree of discrimination has been undertaken.

Correlations are reported where significant association between two variables had been reported in the literature review for the study. In the descriptive results section, correlations are reported that are greater than F .292. Lower levels of correlation are not reported due to the large amount of data unless indicated. Therefore, only those correlations close to or over moderate and with at least 95\% confidence are shown.

\subsubsection{SOCIO-DEMOGRAPHIC DESCRIPTIVE RESULTS}

This chapter sub-section presents descriptive results for respondent socioeconomic data. Results are compared with New Zealand demographic statistics available for this population on the New Zealand Statistics site (NZ Govt) and Report on Maternity (Ministry of Health, 2004), in order to assess whether the sample is representative of New Zealand nulliparous women. The diagram below shows the sub-domain of the conceptual framework in focus highlighted. 


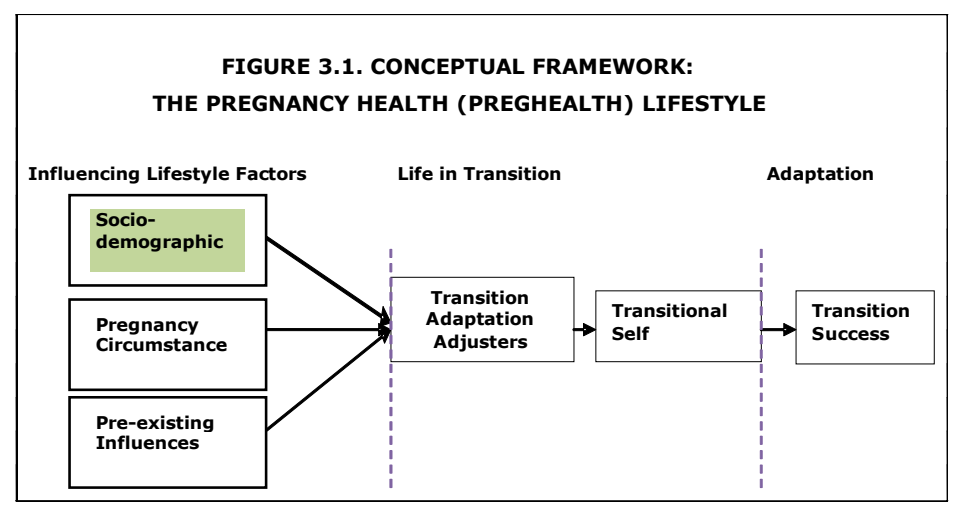

\subsubsection{Age, Ethnicity and Education}

Table 5.2 shows a break down of respondents by age, ethnicity and education.

Table 5.26. Age, Ethnicity and Education

\begin{tabular}{|c|c|c|c|c|c|}
\hline Variable & $\mathbf{n}$ & $\%$ & Variable & $\mathbf{n}$ & $\%$ \\
\hline Age Grouping & & & Education & & \\
\hline $15-19$ & 37 & $7.7 \%$ & College 1-3yrs & 49 & $10.3 \%$ \\
\hline $20-24$ & 70 & $14.6 \%$ & College over 3 years & 98 & $20.5 \%$ \\
\hline $25-29$ & 111 & $23.2 \%$ & Trade cert undergrad dip/ & 110 & $23.0 \%$ \\
\hline $30-34$ & 176 & $36.8 \%$ & Bach or post-grad dip & 161 & $33.7 \%$ \\
\hline $35-39$ & 73 & $15.3 \%$ & Post grad degree & 58 & $12.1 \%$ \\
\hline $40+$ & 11 & $2.3 \%$ & Missing & 2 & $0.4 \%$ \\
\hline Total & 478 & $100 \%$ & Total & 478 & $100 \%$ \\
\hline \multicolumn{6}{|l|}{ Ethnicity* } \\
\hline Pakeha & 308 & $64.4 \%$ & & & \\
\hline Māori & 20 & $4.2 \%$ & & & \\
\hline Māori/Pakeha** & 30 & $6.3 \%$ & & & \\
\hline Pacific/Pakeha*** & 15 & 3.1 & & & \\
\hline British/European & 50 & 10.5 & & & \\
\hline Asian & 26 & 5.4 & & & \\
\hline Other & 28 & 5.9 & & & \\
\hline Missing & 1 & 0.2 & & & \\
\hline Total & 478 & $100 \%$ & & & \\
\hline
\end{tabular}

$*$ Respondents were able to tick more than one category.

**Combined Māori and Māori/Pakeha proportion of $10.5 \%$

***Pacific and Pacific/Pakeha categories combined.

Respondent ages ranged from 15 to 44 years, with a mean of 29 and median of 31. Sixty percent of respondents were between 25-34 years. The largest age group was 30-34 years (37\% of all respondents). Report on Maternity, (Ministry of Health, 2004) reports that average age of NZ mothers as 30.2 years, with $7 \%$ 
of mothers $(n=3845)$ less than 20 years of age. This was not able to be broken into age at first birth or parity.

Within the respondent group, $64.4 \%$ identified themselves as Pakeha. The ethnic representation appears to be broadly acceptable in comparison with the New Zealand population statistics for ethnicity (Statistics New Zealand Census, 2006). Ethnic make up for the total New Zealand population are European 69\% ('Pakeha' in survey), Māori $17 \%$, Asian $8 \%$, Pacific peoples $8 \%$, Other $10 \%$ (NZ Government, Census 2006).

Report on Maternity (Ministry of Health, 2004) reports that the majority of mothers who gave birth in 2003 were European (57.3 percent). The proportion of Māori and Pacific mothers who gave birth (19.8 and 10.5 percent respectively) was higher than the relative proportion of each in the total population of women of reproductive age (15.3 and 6.0 percent respectively) (p. 13). In this study, Pacific women in the survey are slightly below the New Zealand population count for the child-bearing age group. Māori and Pacific women were in the younger age groups in this respondent group. This compares well with Report on Maternity (2004), with Māori and Pacific groups having a lower median age than compared with other ethnic groups, where the median for non-Māori and nonPacific mothers was 30.3 years, compared with 25.8 for Māori and 27.7 for Pacific (although this figure was not available broken down by parity, so age at first birth was not available).

In regard to highest educational achievement, $45.8 \%$ of respondents have a Bachelor degree or above. This means the sample is 'well educated' and probably not educationally representative of the total childbearing population. Statistics New Zealand states that from the 2006 census that in the '20-39' age groups " $80 \%$ have a 'qualification'. Of the total population, $40 \%$ have a post-school qualification. A Bachelors degree is held by $7 \%$ and $5 \%$ hold a higher degree (Statistics New Zealand website). During data analysis it was noted that there were concentrations of occupations, such as 'policy analyst', with an associated Bachelor or above qualification". This is probably a function of the data having been collected primarily in Wellington; as a capital city, Government related work is more prevalent, and Wellington has the highest percentage of degree prepared residents in New Zealand. 
Cross-tabulations of 'Age' by 'Ethnicity' and 'Age' by 'Education' are shown in Tables 5.27 and 5.28. These two tables demonstrate that Māori and Pacific women tended to be younger having their first baby than respondents in other ethnic groups. Collapsed education categories with age groupings shows that the younger a respondent is having a first baby, the fewer educational qualifications have been obtained (although those 15-19 years could not have achieved a University degree at the time of study, based purely on age). The older a respondent is and having a first baby, the more likely she is to have a Bachelor degree or above. Ethnicity and age distribution can be seen more prominently by further condensing ethnic groups as in Table 5.27. Data in this table demonstrates that Māori and Pacific respondents experience their first birth at a younger age than other ethnic groups.

Table 5.27 Age by Ethnicity (reduced categories)

\begin{tabular}{lllll}
\hline Age & Pakeha/European & Maori/Pacific & Asian/Other & Total \\
\hline $15-24$ & 59 & 39 & 9 & 107 \\
& $16.5 \%$ & $60.0 \%$ & $16.7 \%$ & $22.4 \%$ \\
$25-34$ & 231 & 21 & 34 & 286 \\
& $64.5 \%$ & $32.3 \%$ & $63.0 \%$ & $60.0 \%$ \\
$34-40+$ & 68 & 5 & 11 & 84 \\
& $19.0 \%$ & $7.7 \%$ & $20.4 \%$ & $17.6 \%$ \\
Total & 358 & 65 & 54 & 477 \\
& $100.0 \%$ & $100.0 \%$ & $100.0 \%$ & $100.0 \%$ \\
\hline
\end{tabular}

$\mathrm{p}=.000$

Table 5.28. Age by Education

\begin{tabular}{lllllll}
\hline Age group & $\begin{array}{l}\text { College } \\
\text { 1-3yrs }\end{array}$ & $\begin{array}{l}\text { College } \\
\mathbf{3 + y r s}\end{array}$ & $\begin{array}{l}\text { Tradecert } \\
\text { Undergrad Dip }\end{array}$ & $\begin{array}{l}\text { Bach or } \\
\text { Postgrad Dip }\end{array}$ & $\begin{array}{l}\text { Postgrad } \\
\text { degree }\end{array}$ & Total \\
\hline $15-19$ & 20 & 12 & 4 & 0 & 0 & 36 \\
& $40.8 \%$ & $12.2 \%$ & $3.6 \%$ & $0.0 \%$ & $0.0 \%$ & $7.6 \%$ \\
$20-24$ & 13 & 30 & 20 & 6 & 1 & 70 \\
& $26.5 \%$ & $30.6 \%$ & $18.2 \%$ & $3.7 \%$ & $1.7 \%$ & $14.7 \%$ \\
$30-34$ & 4 & 19 & 30 & 46 & 11 & 110 \\
& $8.2 \%$ & $19.4 \%$ & $27.3 \%$ & $28.6 \%$ & $19.0 \%$ & $23.1 \%$ \\
$35-39$ & $16.3 \%$ & $20.4 \%$ & $33.6 \%$ & 81 & 30 & 176 \\
& 3 & 16 & 18 & $50.3 \%$ & $51.7 \%$ & $37.0 \%$ \\
$40+$ & $6.1 \%$ & $16.3 \%$ & $16.4 \%$ & 24 & 12 & 73 \\
& 1 & 1 & 1 & $14.9 \%$ & $20.7 \%$ & $15.3 \%$ \\
Total & 20 & 37 & 4 & 4 & 11 \\
& 49 & 98 & 110 & $2.5 \%$ & $6.9 \%$ & $2.3 \%$ \\
& $100.0 \%$ & $100.0 \%$ & $100.0 \%$ & $100.0 \%$ & $100.0 \%$ & $100.0 \%$ \\
\hline
\end{tabular}

Fisher's Exact Test $p=.000$ 


\subsubsection{Living and Accommodation}

Report on Maternity (2004) specifies most births occur in urban centres (with Counties Manakau the highest). In this respondent group, $81.2 \%$ of respondents live in a 'large' or 'smaller' city'. A total of $86.2 \%$ of respondents live with their partner, although this percentage could be higher, as it is not known how many of those living with their parents also lived with their partner there. Those aged 1524 years are more likely to be living in arrangements other than exclusively with their partner, with $56.7 \%$ of those $15-19$ years living with parent/s or in an extended household (where the partner may or may not also be living). For $91.6 \%$ of respondents, the present expected child would be the only child they would currently have responsibility for (see Table 5.29 below).

Table 5.29. Living and Accommodation

\begin{tabular}{|c|c|c|c|c|c|}
\hline Variable & $\mathbf{n}$ & $\%$ & Variable & $\mathbf{n}$ & $\%$ \\
\hline Region type* & & & Contact with baby's father & & \\
\hline Large city $(>100,000)$ & 302 & $63.20 \%$ & Live together & 433 & $90.6 \%$ \\
\hline Smaller city $\quad(30,000-$ & 86 & $18.00 \%$ & Live apart but regular contact & 21 & $4.4 \%$ \\
\hline 99.999) & & & & & \\
\hline Town $(1,000$ to 29,999$)$ & 69 & $14.40 \%$ & No contact & 17 & $3.6 \%$ \\
\hline $\begin{array}{l}\text { Small town, community or } \\
\text { village ( }<1,000 \text { people) }\end{array}$ & 19 & $4.00 \%$ & Occasional & 7 & $1.5 \%$ \\
\hline Missing & 2 & $0.4 \%$ & Missing & 0 & $0 \%$ \\
\hline Total & 478 & $100 \%$ & Total & 478 & $100 \%$ \\
\hline Living arrangement & & & Care for other children $* * *$ & & \\
\hline With partner & 412 & $86.20 \%$ & Not responsible for any & 438 & $91.60 \%$ \\
\hline With parents & 29 & $6.10 \%$ & 4 days or less & 20 & $4.20 \%$ \\
\hline Extended house/whanau & 15 & $3.10 \%$ & Over 4 days & 18 & $3.80 \%$ \\
\hline On own & 11 & $2.30 \%$ & Missing & 2 & $0.40 \%$ \\
\hline In flat & 11 & $2.30 \%$ & Total & 478 & $100 \%$ \\
\hline Missing & 0 & $0 \%$ & & & \\
\hline Total & 478 & $100 \%$ & & & \\
\hline
\end{tabular}

*Domicile by actual city or town not collected

** Nine respondents ticked more than one category, usually living with partner and parents

$* * *$ Caring for children who may be partners or through adoption process.

\subsubsection{Primary Language at Home}

For $96.7 \%$ of women, the main language spoken at home was English. The next most common language was 'Chinese' and 'Other Asian,' (making up 2.1\% of the total group). Sixty five respondents, (13.6\%) had lived in New Zealand 5 years or less and 18 women (3.8\%) one year or less. New arrivals were mainly 'British 
European' $(n=37)$ and 'Asian' or 'Other'. Pacific Island respondents had all been in New Zealand over 5 years.

\subsubsection{Work and Income}

Table 5.7 shows results for work, occupation and income.

Table 5.30. Work and income

\begin{tabular}{|c|c|c|c|c|c|}
\hline & $\mathbf{n}$ & $\%$ & & $\mathbf{n}$ & $\%$ \\
\hline Occupational group & & & Work cessation & & \\
\hline Clerical & 109 & $22.80 \%$ & Were not working & 77 & $16.1 \%$ \\
\hline Commercial prof/manager & 69 & $14.40 \%$ & Before 27 weeks & 20 & $4.2 \%$ \\
\hline Teaching/health sciences & 68 & $14.20 \%$ & 27-29 weeks & 16 & $3.3 \%$ \\
\hline Home/beneficiary/student & 55 & $11.50 \%$ & 30-32 weeks & 50 & $10.5 \%$ \\
\hline Research/science/technician & 53 & $11.10 \%$ & 33-35 weeks & 82 & $17.2 \%$ \\
\hline Marketing/sales/hospitality & 51 & $10.70 \%$ & 36-38 weeks & 198 & $41.4 \%$ \\
\hline Factory/trade & 40 & $8.40 \%$ & $39-40$ weeks & 25 & $5.2 \%$ \\
\hline Professions & 30 & $6.30 \%$ & Missing & 10 & $2.1 \%$ \\
\hline Missing & 3 & $0.60 \%$ & Total & 478 & $100 \%$ \\
\hline Total & 478 & $100 \%$ & Work 36 wks+*** & $\mathbf{s}$ & \\
\hline Household income $* *$ & & & Professions & 15 & $51.7 \%$ \\
\hline$<19,999 \mathrm{~K}$ & 42 & $8.8 \%$ & $\begin{array}{l}\text { Commercial } \\
\text { prof/manager }\end{array}$ & 42 & $60.9 \%$ \\
\hline $20-29,999$ & 33 & $6.9 \%$ & $\begin{array}{l}\text { Research/science } \\
\text { /technician }\end{array}$ & 32 & $60.4 \%$ \\
\hline $30-39,999$ & 34 & $7.1 \%$ & $\begin{array}{l}\text { Teaching/health } \\
\text { sciences }\end{array}$ & 32 & $47.8 \%$ \\
\hline $40-49,999$ & 43 & $9.0 \%$ & $\begin{array}{l}\text { Marketing/sales } \\
\text { /hospitality }\end{array}$ & 13 & $25.5 \%$ \\
\hline $50-69,999$ & 78 & $16.3 \%$ & Clerical & 89 & $63.9 \%$ \\
\hline $70-99,999$ & 84 & $17.6 \%$ & Factory/trade & 13 & $35.1 \%$ \\
\hline Over 100 & 150 & $32.3 \%$ & $\begin{array}{l}\text { Home/beneficiary } \\
\text { /student }\end{array}$ & 6 & $11.8 \%$ \\
\hline Missing & 14 & $2.9 \%$ & Total work $36+w k s$ & 322 & $47.8 \%$ \\
\hline Total & 478 & $100 \%$ & $\begin{array}{l}\text { (missing from total } \\
\text { sample) }\end{array}$ & 13 & $2.7 \%$ \\
\hline Employment status & & & $\begin{array}{l}\text { Employment status } \\
\text { cont., }\end{array}$ & & \\
\hline Full time & 247 & $51.70 \%$ & Student & 23 & $4.80 \%$ \\
\hline Maternity leave & 74 & $15.50 \%$ & $\begin{array}{l}\text { Unemployed/ seeking } \\
\text { work }\end{array}$ & 7 & $1.50 \%$ \\
\hline Part time & 52 & $10.90 \%$ & Other & 10 & $2.1 \%$ \\
\hline At home & 30 & $6.30 \%$ & Missing & 6 & $1.3 \%$ \\
\hline Sickness benefit & 29 & $6.10 \%$ & Total & 478 & $100 \%$ \\
\hline
\end{tabular}

* More detail of occupation codes and breakdown available on request. Respondents nominated their occupation. NZ Statistics Occupational Grouping codes were initially used to aggregate nominated occupation. However, a female only respondent group meant many occupational and industry 
categories were not relevant and modifications to the classifications were made in order to better represent the range of chiefly feminine occupations.

**Household incomes include wealth generated by partner.

$* * *$ Reported as the percent of each occupational group. For example, $51.7 \%$ of respondents in professional occupational categories work until 36 weeks or more.

Approximately one third, $32.3 \%$ of respondents have a household income of over $\$ 100 \mathrm{~K}$ per year. A cross-tabulation of household income by occupation showed the greater incomes in the top 'professional, managerial, and technical'. Just over half $(51.7 \%)$ of the respondents were still working full time when they undertook the study and $48.7 \%$ had or were planning to work till 36 weeks or more. When age group was cross-tabulated with employment status, findings showed that the younger a respondent was, the more likely she was to be a student or on a sickness benefit. There was no significant difference between the pregnancy risk status and planned or actual gestation of work cessation when cross-tabulated. When work cessation was analysed by occupational group, sales/marketing, hospitality and trade/factory/labourer groups tend to cease work sooner than other occupational groups (excluding 'student' and 'at home').

Māori and Pacific respondents are more likely to live in households with an income less than $\$ 50 \mathrm{~K}$ per year. The New Zealand Deprivation Index reports that a larger concentration of mothers live in areas that are in the lower socioeconomic deciles. As well, these areas tend to be populated more by Māori and Pacific women (Ministry of Health 2004). British/European respondents have the highest household incomes (see Table 5.31).

Table 5.31. Household Yearly Income by Ethnicity (reduced categories)

\begin{tabular}{lllll}
\hline $\begin{array}{l}\text { Household } \\
\text { income }\end{array}$ & Pakeha/European & Maori/Pacific & Asian/other & Total \\
\hline$<29,999 \mathrm{k}$ & 44 & 24 & 6 & 74 \\
$30-49,999 \mathrm{k}$ & $12.60 \%$ & $38.10 \%$ & $11.50 \%$ & $16.00 \%$ \\
& 49 & 13 & 15 & 77 \\
$50-69,999 \mathrm{k}$ & $14.10 \%$ & $20.60 \%$ & $28.80 \%$ & $16.60 \%$ \\
$70-99,999 \mathrm{k}$ & 57 & 9 & 12 & 78 \\
& $16.40 \%$ & $14.30 \%$ & $23.10 \%$ & $16.80 \%$ \\
$>100,000 \mathrm{k}$ & 61 & 12 & 11 & 84 \\
& $17.50 \%$ & $19.00 \%$ & $21.20 \%$ & $18.10 \%$ \\
Total & 137 & 5 & 8 & 150 \\
& $39.40 \%$ & $7.90 \%$ & $15.40 \%$ & $32.40 \%$ \\
\hline$p$ & 348 & 63 & 52 & 463 \\
\hline
\end{tabular}

$\mathrm{p}=.000$ 


\subsubsection{Media use}

Table 5.9 presents respondent 'media usage by frequency'. The Internet and television are media with the highest usage, with $58.3 \%$ of the respondents consider themselves high users of the Internet. As seen in Table 5.33, Internet usage tends to increase with income.

Table 5.32. Media use

\begin{tabular}{lrr}
\hline Type & Mean & SD \\
\hline Television & 5.90 & 1.21 \\
Internet & 5.39 & 1.73 \\
Direct mail & 5.00 & 1.70 \\
DVDs & 4.61 & 1.40 \\
Newspapers & 4.45 & 1.68 \\
Community papers & 4.43 & 1.55 \\
Cinema & 3.91 & 1.36 \\
Women's magazines & 3.87 & 1.65 \\
Lifestyle magazines & 3.23 & 1.67 \\
Business tabloids & 2.42 & 1.51 \\
Playstation & 1.84 & 1.33 \\
\hline
\end{tabular}

Table 5.33. Internet Usage* by Yearly Household Income Groups

\begin{tabular}{lllllll}
\hline Internet Use & $<\mathbf{2 9 , 9 9 9 k}$ & $\mathbf{3 0 -}$ & $\mathbf{5 0 -}$ & $\mathbf{7 0 -}$ & $\mathbf{8 1 0 0 , 0 0 0 k}$ & Total \\
& & $\mathbf{4 9 , 9 9 9 k}$ & $\mathbf{6 9 , 9 9 9 k}$ & $\mathbf{9 9 , 9 9 9 k}$ & & \\
\hline Low & 15 & 13 & 13 & 6 & 10 & 57 \\
& $20.0 \%$ & $16.9 \%$ & $16.9 \%$ & $7.1 \%$ & $6.7 \%$ & $12.3 \%$ \\
Mod & 34 & 24 & 22 & 21 & 35 & 136 \\
& $45.3 \%$ & $31.2 \%$ & $28.6 \%$ & $25.0 \%$ & $23.3 \%$ & $29.4 \%$ \\
High & 26 & 40 & 42 & 57 & 105 & 270 \\
& $34.7 \%$ & $51.9 \%$ & $54.5 \%$ & $67.9 \%$ & $70.0 \%$ & $58.3 \%$ \\
Total & 75 & 77 & 77 & 84 & 150 & 463 \\
& $100.0 \%$ & $100.0 \%$ & $100.0 \%$ & $100.0 \%$ & $100.0 \%$ & $100.0 \%$ \\
\hline $\mathrm{p}=.000$ & & & & & &
\end{tabular}

\subsubsection{Summary of socio-demographic profile and representation}

The respondent profile was largely representative of the population and therefore discussion can proceed with a degree of confidence. Also most of the distribution occurred in Wellington, distribution to several other centres outside of Wellington led to a wide range of response from different geographical region size. Distribution sites, such as youth antenatal classes led to a good representation of women under twenty years of age in the sample, which may have been difficult to gain otherwise. 
A high level of graduate education in the group may have impacted on the scores for some variables. An influencing factor for this is that New Zealand has a higher level of graduates than other countries (Statistics New Zealand n.d.) and increasing age for first pregnancies does mean that the second decade of life is freer for completion of education than in previous decades for women, when the age of first baby was considerably lower.

In regard to ethnic representation, a lower response than population representation was achieved from Pacific women. This response level was achieved despite efforts undertaken in the initial wave of data collection and then again in booster stages. It is not known whether the response level was a factor of low levels of offer by LMC's or non-return from Pacific women holding questionnaire packs.

\subsubsection{PREGNANCY CIRCUMSTANCE DESCRIPTIVE RESULTS}

This section of the data collection presents findings pertaining to the pregnancy circumstance. The diagram below shows the sub-domain of the conceptual framework in focus highlighted

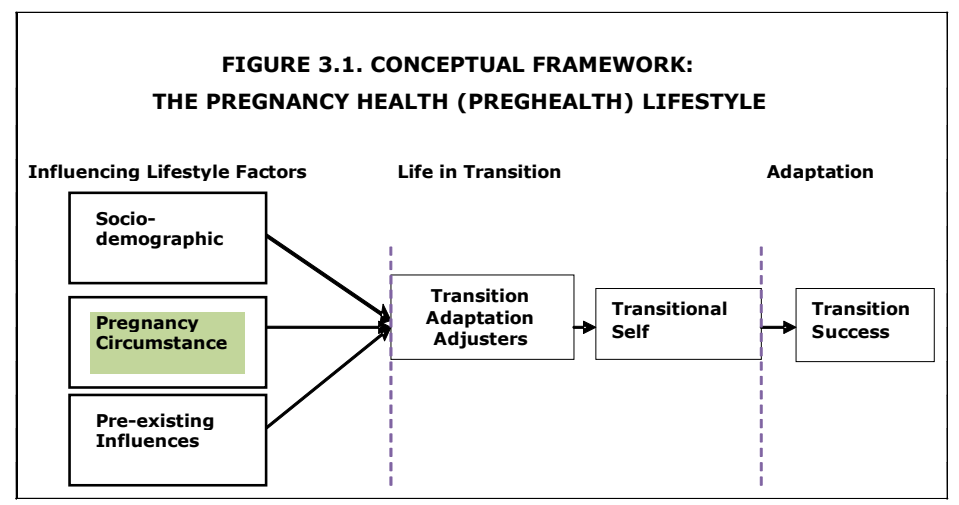

\subsubsection{Feelings about the pregnancy, Pregnancy dates and Other experiential factors.}

This sub-section presents results for the constructs of Pregnancy Circumstance and their variables. These constructs are; 'Feelings about Pregnancy', Pregnancy dates and 'Other experiential factors.

Table 5.34, shows that $70.1 \%$ of the sample had 'planned' pregnancies (this includes 'wanted' and 'wanted to become earlier'). As the minimum time of gestation for this study was 20 weeks, many unwanted pregnancies may have been terminated, so this figure cannot be generalised for all unwanted/unplanned 
pregnancies in the childbearing population. Most women rated their pregnancy as a positive life event.

Table 5. 34. Feelings about the Pregnancy, Pregnancy Dates and Other Experiential Factors.

\begin{tabular}{|c|c|c|c|c|c|}
\hline Variable & $\mathbf{n}$ & $\%$ & Variable & $\mathbf{n}$ & $\%$ \\
\hline Preg. Intendedness & & & First awareness & of & preg \\
\hline Wanted to get pregnant & 259 & $54.20 \%$ & By 6 weeks & 379 & $79.3 \%$ \\
\hline Not right away & 127 & $26.60 \%$ & 7 to 12 weeks & 89 & 18.65 \\
\hline Wanted earlier & 76 & $15.90 \%$ & 13 to 20 weeks & 8 & $1.7 \%$ \\
\hline Did not want & 16 & $3.30 \%$ & After 20 weeks & 2 & $0.4 \%$ \\
\hline Total & 478 & $100 \%$ & Total & 478 & $100 \%$ \\
\hline $\begin{array}{l}\text { Original intentions for } \\
\text { the pregnancy } * * *\end{array}$ & & & Knows sex of baby & & \\
\hline Never & 385 & $80.50 \%$ & Yes & 265 & $55.4 \%$ \\
\hline For a moment & 50 & $10.50 \%$ & No & 213 & $44.6 \%$ \\
\hline Frequently & 24 & $5.00 \%$ & Total & 478 & $100 \%$ \\
\hline Occasionally & 19 & $4.00 \%$ & Previously pregnant & & \\
\hline Total & 478 & $100 \%$ & Yes & 144 & $30.1 \%$ \\
\hline Eval. of preg. worth & & & Missing & 1 & $0.2 \%$ \\
\hline Really wants & 405 & $84.7 \%$ & Total & 478 & $100 \%$ \\
\hline Is $\mathrm{OK}$ & 71 & $14.9 \%$ & Prev. pregnant by & age & (n 144) \\
\hline Not wanting & 2 & $0.4 \%$ & $15-19$ & 10 & $27.0 \%$ \\
\hline Total & 478 & $100 \%$ & $20-24$ & 27 & $38.6 \%$ \\
\hline Rating as life event $* *$ & & & $25-29$ & 31 & $28.2 \%$ \\
\hline Worst thing-5 & 19 & $3.9 \%$ & $30-34$ & 50 & $28.4 \%$ \\
\hline 6 & 24 & $5.0 \%$ & $35-39$ & 23 & $31.5 \%$ \\
\hline 7 & 58 & $12.1 \%$ & $40+$ & 3 & $27.3 \%$ \\
\hline 8 & 120 & $25.1 \%$ & Total & 144 & $100 \%$ \\
\hline 9 & 123 & $25.7 \%$ & Weeks now & & \\
\hline Best thing & 133 & $27.8 \%$ & 25 or below & 107 & $22.4 \%$ \\
\hline Missing & 1 & $0.2 \%$ & $26-30$ & 133 & $27.8 \%$ \\
\hline Total & 478 & $100 \%$ & $31-35$ & 152 & $31.8 \%$ \\
\hline Fertility assistance* & & & 36 or above & 86 & $18.0 \%$ \\
\hline
\end{tabular}




\begin{tabular}{llllll}
\hline Variable & $\mathbf{n}$ & $\mathbf{\%}$ & Variable & $\mathbf{n}$ & $\mathbf{\%}$ \\
\hline $\begin{array}{l}\text { Advice/med tests } \\
(n=140)\end{array}$ & 64 & $13.4 \%$ & Total & 478 & $100 \%$ \\
Ovulation meds $(n=133)$ & 19 & $4 \%$ & & \\
Artificial insem $(n=133)$ & 6 & $1.3 \%$ & & \\
IVF $(n=133)$ & 15 & $3.1 \%$ & & \\
Other $(n=133)$ & 15 & $3.1 \%$ & & \\
\hline
\end{tabular}

*As at time of pregnancy occurrence

** Rating as a life event categories Worst-5 scores combined due to the small number of responses. Mean for this item is 8.44 and the Median 9.

*** In response to whether they considered ending the pregnancy

In regard to previous pregnancy, 333 respondents $(69.7 \%)$ had not been pregnant before, with 144 (30.1\%) having had an earlier pregnancy (in this study women who had not passed 24 weeks in a prior pregnancy were within the inclusion criteria). There was no differentiation made between spontaneous or induced abortion in the questionnaire due to potential sensitivities. The percentage rate of previous pregnancies to no previous pregnancies showed no significant difference among all age groups, even within the 15-19 year group.

Table 5.35 shows Pearson's correlations with 'Rating as a life event' and items from the Psychosocial and Transition Success sub-domains. There is a moderate degree of positive correlation.

Table 5.35. Rating as a life event: Pearson's correlations**

\begin{tabular}{lll}
\hline Item/scale & with & r \\
\hline Rating as a life event & Body satisfaction & .419 \\
& Dimensions of wellness & .326 \\
& Looking forward to a new life stage & .420 \\
& Happy to leave childless days behind & .354 \\
\hline
\end{tabular}

** All Pearson's correlations reported here significant at the .01 level (two tailed)

Table 5.36. shows that respondents in the 'Pregnancy intendedness' categories of 'wanted' or 'wanted to become earlier' have higher levels of early awareness (0-6 weeks) than 'did not want' or 'wanted but not right away'. 
Table 5.36. First awareness by Pregnancy intendedness

\begin{tabular}{llllll}
\hline Weeks & Wanted & $\begin{array}{l}\text { Wanted to } \\
\text { become earlier }\end{array}$ & $\begin{array}{l}\text { Not right } \\
\text { away }\end{array}$ & $\begin{array}{l}\text { Did not } \\
\text { want }\end{array}$ & Total \\
\hline $0-6$ & 229 & 66 & 75 & 9 & 379 \\
& $60.4 \%$ & $17.4 \%$ & $19.8 \%$ & $2.4 \%$ & $100.0 \%$ \\
$7-12$ & 30 & 9 & 45 & 5 & 89 \\
& $33+$ & $10.1 \%$ & $50.6 \%$ & $5.6 \%$ & $100.0 \%$ \\
& 0 & 1 & 7 & 2 & 10 \\
Total & $0.0 \%$ & $10.0 \%$ & $70.0 \%$ & $20.0 \%$ & $100.0 \%$ \\
& 259 & 76 & 127 & 16 & 478 \\
& $54.2 \%$ & $15.9 \%$ & $26.6 \%$ & $3.3 \%$ & $100.0 \%$ \\
\hline
\end{tabular}

Fisher's Exact Test p .001

Table 5.37. shows that 'wanted but not right away' has a higher level of 'is OK' than 'wanted' or 'wanted earlier'. 'Rating as a life event' (collapsed into 3 groups) was also cross-tabulated with 'Pregnancy intendedness'. The 'wanted to become pregnant earlier' group has the highest level of pregnancy appraisal scores (810).

Table 5.37 Pregnancy intendedness by Evaluation of pregnancy worth

\begin{tabular}{llllll}
\hline Evaluation & Wanted & Wanted earlier & Not right away & Did not want & Total \\
\hline Really wants & 239 & 74 & 86 & 6 & 405 \\
& $92.3 \%$ & $97.4 \%$ & $67.7 \%$ & $37.5 \%$ & $84.7 \%$ \\
Is OK & 20 & 2 & 40 & 9 & 71 \\
& $7.7 \%$ & $2.6 \%$ & $31.5 \%$ & $56.3 \%$ & $14.9 \%$ \\
Not wanting & 0 & 0 & 1 & 1 & 2 \\
& $0.0 \%$ & $0.0 \%$ & $0.8 \%$ & $6.3 \%$ & $0.4 \%$ \\
Total & 259 & 76 & 127 & 16 & 478 \\
& $100.0 \%$ & $100.0 \%$ & $100.0 \%$ & $100.0 \%$ & $100.0 \%$ \\
\hline
\end{tabular}

Fisher's Exact Test p .000

\subsubsection{Arranging pregnancy care}

The results presented here are assigned to the 'Arranging pregnancy care' variable of the 'Information processing and utilisation and Utilisation' construct of the Transition Adaptation Adjusters sub-domain. However, for flow and ease of reading, they are presented with 'Pregnancy Circumstance' results. Table 5.38 shows that $86.2 \%$ of respondents intended to give birth in a hospital and, when a birthing unit is included, the figure is $91.2 \% ; 5.6 \%$ intend to give birth at home, and $69.2 \%$ of respondents chose a Midwife as their LMC. These figures are consistent with current New Zealand statistics where approximately $7 \%$ of births are recorded as occurring at home and $70 \%$ of primary birth is under LMC Midwife care (Ministry of Health, 2004). Nearly all respondents (98.7\%) had had 
a scan. Of the five respondents who had not had a scan, three were in the earliest gestational bracket and two were planning a homebirth. Using a personal recommendation from a friend or contact was the most often used way to engage an LMC, with $42.3 \%$ using it alone or in combination with other search behaviours. This could be expected somewhat due to the high degree of credence a personal source may have for women who have had no previous experience in the area. Responding to an advertisement was the least used.

For issues obtaining care, there was a range of responses for the item 'hard to find a midwife who was unbooked', with a spread across responses (42.8\% of respondents 'agree a little' to 'strongly agree' that it was 'hard to find an unbooked midwife'). A cross tabulation of age group by 'hard to find a midwife who was unbooked' found no significant difference. Those in cities find it more difficult to find an unbooked midwife than those in towns. For other items, $49 \%$ essentially disagree that they have a lot of choice. Most report feeling 'comfortable' with their carer. A substantial number of respondents do not find there is clear information on how to obtain an LMC $(43.4 \%$ fall into agreement categories). In regard to the item 'there was lack of clear information regarding care', there is no relationship found with any other variable, including 'Ethnicity', 'Age', 'Education', 'Health Consumerism', 'Mastery' and 'Health Locus of Control'.

Table 5.38. Arranging pregnancy care

\begin{tabular}{|c|c|c|c|c|c|}
\hline & $\mathbf{n}$ & $\%$ & & $\mathbf{n}$ & $\%$ \\
\hline Intended place & of & birth & Engaging LMC care* & & \\
\hline At home & 27 & $5.6 \%$ & Personal recommendation & 202 & $42.3 \%$ \\
\hline Hospital & 412 & $86.2 \%$ & LMC contact list & 125 & $26.2 \%$ \\
\hline Birthing centre & 24 & $5.0 \%$ & Pamphlet & 114 & $23.8 \%$ \\
\hline Undecided & 15 & $3.1 \%$ & $\begin{array}{l}\text { Referral to GP or med } \\
\text { specialist }\end{array}$ & 73 & $15.3 \%$ \\
\hline Total & 478 & $100 \%$ & Listing in telephone book & 52 & $10.9 \%$ \\
\hline LMC & & & 0800 number & 50 & $10.5 \%$ \\
\hline Midwife & 331 & $69.20 \%$ & Attached to GP's rooms & 44 & $9.2 \%$ \\
\hline $\begin{array}{l}\text { MW/ Private } \\
\text { obstetrician }\end{array}$ & 71 & $14.90 \%$ & Info on website & 43 & $9 \%$ \\
\hline DHB team & 39 & $8.20 \%$ & Conducted interviews & 23 & $4.8 \%$ \\
\hline MW/GP & 36 & $7.50 \%$ & Advertisement & 14 & $2.9 \%$ \\
\hline Not sure & 1 & 0.2 & Other & 32 & $6.7 \%$ \\
\hline
\end{tabular}




\begin{tabular}{llllll}
\hline & $\mathbf{n}$ & $\mathbf{\%}$ & & $\mathbf{n}$ & $\mathbf{\%}$ \\
\hline Midwife & 331 & $69.20 \%$ & Issues arranging care & Mean & SD \\
Had a U/S scan & & & Hard to find unbooked & 3.97 & 2.22 \\
Yes & 472 & 98.7 & Uncomfortable & 2.81 & 1.68 \\
No & 5 & 1.0 & Found a lot of choice & 3.39 & 1.88 \\
Missing & 1 & 0.2 & Felt unsure how to & 3.63 & 1.88 \\
Total & 478 & $100 \%$ & Found process stressful & 3.27 & 1.95 \\
& & & Unable to get type & 2.45 & 1.74 \\
& & & Clear info available & 4.26 & 1.88 \\
\hline
\end{tabular}

*More than one treatment category may have been ticked by respondents.

\subsubsection{Risk}

This subsection presents results for pregnancy risk. Table 5.39 shows that the percentage of high risk pregnancies in the sample is $13.9 \%$. The most common reason for a high risk status is a pre-existing medical condition and then pregnancy related problems. It is not known how many classify their risk as high due to physical, mental or social reasons. A high risk status is often determined after a first pregnancy and birth, as a result of occurrences in the first pregnancy and/or birth event. For that reason, the incidence of reported high risk in this sample may be lower than the overall incidence of high risk in childbearing women, due to a primiparous sample. As many respondents at time of response have not reached the third trimester of their pregnancy where hypertensionrelated problems such as pre-eclampsia present most, this may also be influential in the result.

Table 5. 39. Level of risk

\begin{tabular}{llllll}
\hline Variable & $\mathbf{n = 4 7 8}$ & $\mathbf{\%} *$ & Variable & $\mathbf{n = 6 6}$ & $\mathbf{\%}$ \\
\hline Considered & high & risk* & Reason for high risk*** & & \\
Yes & 66 & $13.9 \%$ & Pre-existing medical & 23 & $33 \%$ \\
No & 338 & $81.2 \%$ & Current pregnancy & 21 & $31 \%$ \\
Not sure & 22 & $4.6 \%$ & Baby's develop or health & 12 & $18 \%$ \\
Missing & 2 & $0.4 \%$ & New medical problem & 7 & $10 \%$ \\
Total & 478 & $100 \%$ & Previous pregnancy & 6 & $9 \%$ \\
& & & Other**** & 14 & $21 \%$ \\
\hline
\end{tabular}

* Could tick multiple options

$* *$ Were asked to specify. Most common reason given was twins 
Table 5. 40 and 5.41 show that the greater percentage of high risk respondents are in the care of a hospital obstetrician. This is not unexpected, as high risk care is secondary level care.

Table 5 40. Level of risk by LMC

\begin{tabular}{lllll}
\hline LMC & No & Yes & Not sure & Total \\
\hline Midwife & 290 & 26 & 13 & 329 \\
& $74.7 \%$ & $39.4 \%$ & $59.1 \%$ & $69.1 \%$ \\
Midwife/GP & 28 & 4 & 4 & 36 \\
Midwife and private & 58 & 13 & 0 & $7.6 \%$ \\
obstetrician & $14.9 \%$ & $19.7 \%$ & $0.0 \%$ & 71 \\
Midwife and hospital & 12 & 23 & 4 & $14.9 \%$ \\
obstetrician & $3.1 \%$ & $34.8 \%$ & $18.2 \%$ & $8.2 \%$ \\
Not sure & 0 & 0 & 1 & 1 \\
& $0.0 \%$ & $0.0 \%$ & $4.5 \%$ & $0.2 \%$ \\
Total & 388 & 66 & 22 & 476 \\
\hline
\end{tabular}

Fisher's Exact Test .000.

Table 5.41 shows that the incidence of risk is increased with age. Highest respondent uncertainty of risk status is occurring in the youngest age groups. Fifty nine percent of hospital obstetric care is high risk (which is not an unexpected finding due to the requirement of obstetrician LMC care for secondary level and above care women). Note the reverse effect for care with a private obstetrician.

Table 5.41. Level of risk by Age group

\begin{tabular}{lllll}
\hline $\begin{array}{l}\text { Awareness of } \\
\text { risk }\end{array}$ & $\mathbf{1 5 - 2 4}$ & $\mathbf{2 5 - 3 4}$ & $\mathbf{3 4 - 4 0 +}$ & Total \\
\hline No & 82 & 247 & 59 & 388 \\
& $78.10 \%$ & $86.10 \%$ & $70.20 \%$ & $81.50 \%$ \\
Yes & 8 & 36 & 22 & 66 \\
& $7.60 \%$ & $12.50 \%$ & $26.20 \%$ & $13.90 \%$ \\
Not sure & 15 & 4 & 3 & 22 \\
& $14.30 \%$ & $1.40 \%$ & $3.60 \%$ & $4.60 \%$ \\
Total & 105 & 287 & 84 & 476 \\
& $100.00 \%$ & $100.00 \%$ & $100.00 \%$ & $100.00 \%$ \\
\hline
\end{tabular}

Fisher's Exact Test .000 . 


\subsubsection{Summary of descriptive results for Pregnancy Circumstance}

Most pregnancies (70\%) are planned and despite many mistimed (unplanned) pregnancies, almost $85 \%$ of all respondents 'really want' their pregnancy at the time of response. Almost $70 \%$ of respondents have not experienced a pregnancy prior to the present one. Women are aware that they are pregnant early in the gestation and by 12 weeks, $80 \%$ are aware. The knowledge of the baby's sex is split approximately $50 / 50$ in the sample. Almost all women elect for a hospital birth, with almost $70 \%$ engaging 'midwife only' care. Personal recommendation is used by almost half the women to engage an LMC, whether alone or in combination with other factors. Ultrasound scanning has been conducted on nearly all of the respondents. Less that $15 \%$ of the women are high risk, with reporting of risk seen more as age increases.

\subsubsection{PRE EXISTING INFLUENCES DESCRIPTIVE RESULTS}

This section presents results for the sub-domain of 'Pre-existing influences' that assessed pre-existing values and traits. The sub-domain of the conceptual framework in focus is highlighted below.

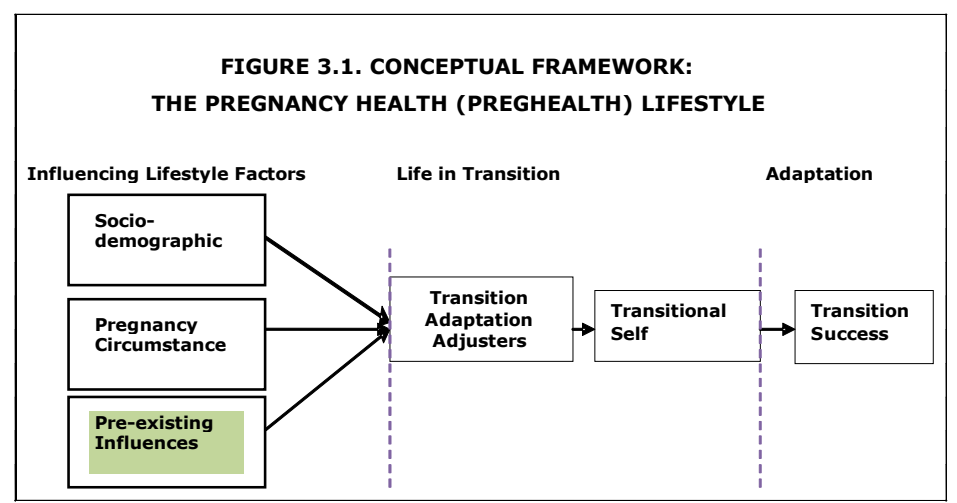

\subsubsection{Pre-existing Health, Mastery and Achievement and Other values, traits and beliefs}

Table 5.42 presents a summary table of findings from this section with commentary. Respondents were asked to respond to the questions are believed they were prior to the pregnancy. 
Table 5 42. Pre-existing Health, Mastery and Achievement and Other values, traits and beliefs

\begin{tabular}{|c|c|c|c|}
\hline $\begin{array}{l}\text { Item /scale (scales are } \\
\text { entered in italics) }\end{array}$ & Mean & SD & Comment \\
\hline \multicolumn{4}{|l|}{ Pre-existing Health } \\
\hline Health as a value & 5.15 & 1.57 & Most respondents value good health highly \\
\hline Health consumerism & 5.43 & 1.06 & \\
\hline Seeks health information & 4.92 & 1.48 & (retained as a single item from CFA Health Consumerism) \\
\hline Pre-pregnancy health level & 5.23 & 1.04 & $\begin{array}{l}\text { 'Level of fitness' and 'Time put in' have the lowest sample means for single items within the } \\
\text { scale. }\end{array}$ \\
\hline \multicolumn{4}{|l|}{ Mastery \& Achievement } \\
\hline Sense of mastery & 5.41 & 1.14 & \\
\hline Feeling toward change & 3.70 & 1.40 & $\begin{array}{l}\text { Most consider themselves quite tolerant of change }(50.4 \% \text { disagree a little or neither } \\
\text { agree/disagree) that they 'don't really like change'. }\end{array}$ \\
\hline \multicolumn{4}{|l|}{ Other Values/Traits } \\
\hline Trait anxiety & 3.61 & 1.42 & \\
\hline Universal Values & & & See Table 5.43 \\
\hline Time orientation & 4.93 & 1.54 & $\begin{array}{l}\text { Most respondents like to plan ahead with } 67.1 \% \text { in the 'agree a little' to 'strongly agree' } \\
\text { categories }\end{array}$ \\
\hline Materialism & 3.92 & 1.53 & Most respondents fall into agree/not agree or agree a little. \\
\hline Environmentalism & 4.68 & 1.14 & $\begin{array}{l}\text { Most agree environmentalism is important. The highest sample mean is for environmental } \\
\text { issues are important (5.14), with decreasing means for 'Buying products that are } \\
\text { environmentally friendly' (4.69) and 'Would not use a product } \\
\text { that damaged the environment' (4.23). }\end{array}$ \\
\hline Concern for physical appearance & 4.53 & 1.23 & \\
\hline Religiosity & 3.00 & 1.90 & $\begin{array}{l}\text { Approx } 73 \% \text { claim to be not religious (disagree a little, to strongly disagree on both items in } \\
\text { scale). There is no correlation between 'Health as a value' and 'Religiosity'. }\end{array}$ \\
\hline
\end{tabular}




\begin{tabular}{|c|c|c|c|}
\hline $\begin{array}{l}\text { Item /scale (scales are } \\
\text { entered in italics) }\end{array}$ & Mean & SD & Comment \\
\hline Cultural strength & 4.88 & 1.48 & $\begin{array}{l}\text { Cultural strength is important for most respondents with } 38.0 \% \text { recording it as very } \\
\text { important in their lives. A cross tabulation with ethnicity showed no significant difference } \\
\text { found between groups. }\end{array}$ \\
\hline Superstition & 3.22 & 1.70 & $\begin{array}{l}\text { This item showed a mixed response. Whilst } 50.2 \% \text { would not agree that they are } \\
\text { superstitious, } 41.8 \% \text { are in the mid-range and } 8 \% \text { feel they are strongly superstitious. }\end{array}$ \\
\hline \multicolumn{4}{|l|}{ Female role items: } \\
\hline Can achieve anything & 6.20 & 1.01 & Strong agreement with statement \\
\hline Balance a major issue & 6.06 & 1.1 .5 & Strong agreement with statement \\
\hline Right to be independent & 6.39 & 0.93 & Strong agreement with statement \\
\hline $\begin{array}{l}\text { Important to have career and } \\
\text { family }\end{array}$ & 4.56 & 1.49 & Most respondent variability from female role items \\
\hline Children most impt role & 3.52 & 1.70 & Most respondents variability from female role items \\
\hline Familism & 3.56 & 0.76 & $\begin{array}{l}\text { Higher means are not for 'obeying' and 'reputation', but more for 'helping with problems'. } \\
\text { Pakeha have the lowest mean for familism and Asian respondents the highest. No significant } \\
\text { difference was found across age groups }\end{array}$ \\
\hline
\end{tabular}


Table 5.43. Kahle's List of Values* (Universal values)

\begin{tabular}{lll}
\hline Value & Mean & SD \\
\hline Relationships & 8.26 & 1.08 \\
Self-respect & 8.25 & 1.09 \\
Fun and enjoyment & 8.12 & 1.1 \\
Security & 8.02 & 1.12 \\
Accomplishment & 7.83 & 1.2 \\
Self-fulfilment & 7.83 & 1.29 \\
Being well respected & 7.67 & 1.28 \\
Belonging & 7.49 & 1.51 \\
Excitement $* *$ & 6.84 & 1.42 \\
\hline
\end{tabular}

*Measured on 9 point Likert scale

Kahle's List of Values showed high means for all items. 'Excitement' was found to have no correlation with 'Health as a Value' or 'Time Orientation'. This item was tested for association with the two latter variables as previous authors had found positive correlations between them.

\subsubsection{Pearson's Correlations with Pre-existing Influences}

Several of the variables are correlated. In particular, 'Pre-pregnancy health level 'is positively correlated to several variables from other conceptual sub-domains; in particular, it has a strong positive correlation with 'Good nutrition effort'. Trait anxiety is positively correlated with other anxiety or concern situations and is strongly correlated with 'Sense of Mastery' in a negative correlation with. 'Concern for physical appearance', 'Materialism', 'Celebrity attraction' and 'Weight concerns' are also associated.

Positive and negative correlations 290 and over are presented in Table 5.44.

Table 5.44. Pre-existing influences: Pearson's correlations**

\begin{tabular}{llll}
\hline items/scales & Same sub-domain & Other sub-domains & r \\
\hline $\begin{array}{l}\text { Pre-pregnancy } \\
\text { health level }\end{array}$ & Time orientation & & .295 \\
& & & .384 \\
& Sense of mastery & & .336 \\
& Trait anxiety & Information processing & and \\
& & utilisation self-efficacy & .354 \\
& & Good nutrition effort & .507 \\
& & Attachment & .384 \\
Sense of mastery & Dimensions of wellness & .387 \\
& & & .384 \\
& & Information receipt interest & .374 \\
& & Dimensions of wellness & .380 \\
\hline
\end{tabular}




\begin{tabular}{llll}
\hline items/scales & Same sub-domain & Other sub-domains & $\mathbf{r}$ \\
\hline Environmentalism & & Good nutrition effort & .369 \\
Concern for & Materialism & & .443 \\
physical & & & .300 \\
appearance & Weight concerns & .323 \\
& Celebrity attraction & .323 \\
Trait anxiety & Superstition & .343 \\
& Feeling toward change & -.408 \\
& Sense of mastery & .339 \\
& Pregnancy state anxiety & .306 \\
\hline
\end{tabular}

** All Pearson's correlations reported here significant at the .01 level (two tailed).

\subsubsection{Summary of Pre-existing Influences descriptive results}

For the respondent group, variables related to health are rated highly. The group is not religious overall. They feel strongly about women's independence and ability to achieve.

\subsubsection{TRANSITION ADAPTATION ADJUSTERS DESCRIPTIVE RESULTS}

This chapter section presents descriptive results for the Transition Adaptation Adjusters sub-domain. The variables within this sub-section include those pertaining to the constructs; 'Information Acquisition', 'Information processing and utilisation and Utilisation' and 'Other Attitudes and Drivers' that are specifically pregnancy-related. The sub-domain of the conceptual framework in focus is highlighted below.

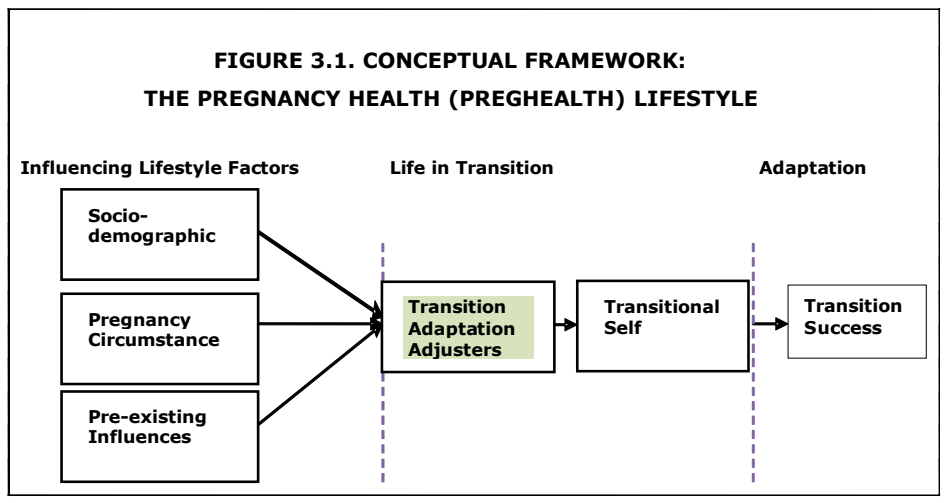

5.4.4.1. Information Acquisition and Information processing and utilisation \& Utilisation

Table 5.45 shows that there were high means for information scales and items. 'Intuition', 'listening to one's body' and 'life experience' are highly regarded as legitimate ways of knowing. There is little resistance to the concept of the value of information. The 'need to balance information with intuition' is very high. There is a higher sample 
mean for 'conflicting information' item than for other items within the 'difficulty and barriers to processing variable group of items.

In regard to the usefulness of sources of information, the midwife and doctor were the most useful sources of information. Medical tests were also highly rated, then pamphlets, then books. Family, friends and mothers were also regarded relatively highly, with little difference between each. Response to the usefulness of partners as an information source showed quite a lot of variation. For those who used the Internet, the search function was regarded as much more useful than chat rooms. Radio documentaries, specialist magazines and lifestyle magazines were of average to low usefulness. Celebrities and soap operas were not regarded as useful sources. Information from strangers and acquaintances had a spread of perceived usefulness. Regarding other health professionals and alternative therapists, these sources were in general infrequently used, with those who did use them reporting low levels of usefulness in general. There was a 'not relevant' option to the question, but responses may also reflect usage as an evaluation of usefulness.

Table 5 45. Information Acquisition and Information processing and utilisation and Utilisation

\begin{tabular}{lll}
\hline Item/scale & Mean & SD \\
\hline Information Acquisition & & \\
Information receipt interest & 6.00 & 0.98 \\
Ways of knowing & & \\
Need to balance with intuition & 5.65 & 1.28 \\
Own body & 4.85 & 1.32 \\
Life experience & 4.39 & 1.56 \\
Vicarious & 3.96 & 1.71 \\
Medical best & 3.58 & 1.46 \\
Usefulness of sources & & \\
Midwife/Dr & 6.61 & 0.84 \\
Medical tests shared by LMC & 6.45 & 0.99 \\
Pamphlets & 6.18 & 1.05 \\
Pregnancy reference book & 5.89 & 1.60 \\
Other family and friends & 5.27 & 1.43 \\
Internet search & 4.89 & 2.20 \\
Mother & 4.77 & 1.97 \\
Antenatal classes & 4.41 & 2.86 \\
TV documentaries & 4.29 & 2.25 \\
Specialist magazines & 3.93 & 2.51 \\
Partner & 3.90 & 2.17 \\
Strangers or acquaintances & 2.86 & 1.10 \\
Radio or TV news item & 2.81 & 2.31 \\
\hline & & \\
& & \\
\hline
\end{tabular}




\begin{tabular}{|c|c|c|}
\hline Item/scale & Mean & SD \\
\hline Lifestyle magazines & 2.55 & 2.24 \\
\hline Dentist & 2.46 & 2.30 \\
\hline Alternative therapists & 2.2 & 2.46 \\
\hline Internet chat rooms & 1.69 & 2.01 \\
\hline Celebrities & 1.61 & 1.61 \\
\hline Yoga instructor & 1.41 & 2.30 \\
\hline Physiotherapist & 1.39 & 2.32 \\
\hline Gym instructor & 1.28 & 2.07 \\
\hline Dietician & 1.24 & 2.20 \\
\hline Soap opera & 1.17 & 1.31 \\
\hline $\begin{array}{l}\text { Information processing and } \\
\text { utilisation }\end{array}$ & & \\
\hline $\begin{array}{l}\text { Information processing and } \\
\text { utilisation self efficacy }\end{array}$ & 5.79 & 0.96 \\
\hline Confident to ask questions & 6.35 & 0.92 \\
\hline Sufficient to make decisions & 5.65 & 1.18 \\
\hline $\begin{array}{l}\text { Perceived information benefits and } \\
\text { outcomes }\end{array}$ & & \\
\hline $\begin{array}{l}\text { Perceived positive information } \\
\text { outcomes }\end{array}$ & 5.77 & 0.80 \\
\hline $\begin{array}{llll}\text { Difficulties } & \& & \text { barriers } & \text { to } \\
\text { processing } & & & \end{array}$ & & \\
\hline Conflicting information & 4.38 & 1.56 \\
\hline Too much can be a bad thing & 3.84 & 1.79 \\
\hline Confused by choices & 3.14 & 1.53 \\
\hline Difficult to make decisions after info & 2.56 & 1.43 \\
\hline Difficult obtaining easily understood & 2.46 & 1.44 \\
\hline Resistance to information & & \\
\hline No interest in changing as result & 2.10 & 1.32 \\
\hline Too many restrictions by LMC & 2.07 & 1.25 \\
\hline Info doesn't make interested in change & 2.02 & 1.20 \\
\hline Change as a result of information & 5.36 & 1.07 \\
\hline Arranging pregnancy care & & \\
\hline See Table 5.38 & & \\
\hline
\end{tabular}

Table 5.45a shows that labour and birth and care of the baby after birth are considered important topics. Getting the father involved is also seen as an important function of antenatal classes. 
Table 5.45a. Antenatal classes* and Topic importance

\begin{tabular}{llllll}
\hline Type & $\mathbf{n}$ & $\mathbf{\%}$ & Importance of topics & Mean & SD \\
\hline Classes by agency & 201 & 42.1 & Care of baby after birth & 6.56 & 0.98 \\
Offered by hospital & 155 & 32.4 & Labour and birth & 6.45 & 1.01 \\
Midwife group & 151 & 31.6 & Baby feeding & 6.42 & 1.19 \\
Doesn't want & 37 & 7.7 & Father's involved & 5.08 & 1.45 \\
Specialist topic & 23 & 4.8 & Caring for baby in pregnancy & 5.88 & 1.59 \\
Midwife one to one & 14 & 2.9 & Caring for self in pregnancy & 5.54 & 1.65 \\
Classes fully booked & 9 & 1.9 & Meeting others & 5.43 & 1.67 \\
\end{tabular}

*Could tick more than one option

\subsubsection{Other Pregnancy Attitudes and Drivers}

Table 5.46 shows a high sample mean for 'Pregnancy should be treated as a natural event'. Pearson's correlation between 'Trust nature' and 'Natural event' $r=0.287$ ( $p .=$ 0.01 ) are positively correlated. However, there is a high level of affinity for technology, with respondents believing 'You should trust nature rather than technology' to assess how a pregnancy is going. However, it is not known whether there was a universal understanding of the meaning of 'technology'.

As seen in the Table 5.46, there is a high mean for 'Needing a scan' and 'Technology makes pregnancy safer'. For ultra-sound scanning, higher means for needing a scan were 'To see if the baby was $\mathrm{OK}^{\prime}$ ', is significantly higher at $95 \%$ confidence that any other item. 'To see when the baby was due' was also scored highly. Items relating to validation of a real baby also show high means, with inclusion of the partner as well in this.

Table 5.46 shows a moderately high Internal Health Locus of Control. However single items retained from the discarded EHLOC subscale have high means. This may be indicative of the 'Partnership' Midwifery Model used in New Zealand, as the items with highest means are ones that are consultative. Additionally, although pregnancy is also a normal healthy event for most women, it does involve close interaction with a health system as an expected component of caring for oneself and baby.

Overall respondents have a very high 'desire to be involved in decisions' regarding their pregnancy health, with $86 \%$ of respondents marking in the 'disagree a lot/strongly disagree' boxes to not knowing if something was wrong. The item regarding the notion that pregnant women can 'Live unhealthily and still have a healthy baby' was dropped during exploratory factor analysis. This item was compared with 'smoking profile' and 
'effort put into health'. Both measures of association were not significantly different to zero.

Table 5.46 Affinity for technology, Pregnancy Health Locus of Control, Pregnancy health motivation and effort, Achievement motivation

\begin{tabular}{|c|c|c|}
\hline Item/scale & Mean & SD \\
\hline \multicolumn{3}{|l|}{ Technology versus nature } \\
\hline Treat as a natural event & 5.73 & 1.25 \\
\hline Technology makes birth safer & 5.69 & 1.27 \\
\hline Need a scan & 5.2 & 1.69 \\
\hline Trust nature & 3.3 & 1.51 \\
\hline \multicolumn{3}{|l|}{ Reasons for having a scan } \\
\hline See if baby is Ok & 6.85 & 0.51 \\
\hline Enable the partner to see the baby & 5.53 & 1.70 \\
\hline See when baby due & 5.26 & 1.63 \\
\hline See if the baby is real & 5.22 & 1.86 \\
\hline Obtain a photo & 4.27 & 2.00 \\
\hline Increase feelings for the baby & 4.25 & 2.08 \\
\hline Learn sex of baby & 3.12 & 1.99 \\
\hline \multicolumn{3}{|l|}{ Health Locus of Control } \\
\hline Internal HLOC & 4.65 & 0.93 \\
\hline External HLOC: Fate & 3.81 & 1.16 \\
\hline Live unhealthily and still having a healthy baby* & 4.08 & 1.59 \\
\hline \multicolumn{3}{|l|}{ Eternal HLOC Powerful others items $* *$} \\
\hline - Must follow advice of Dr or midwife & 5.14 & 1.24 \\
\hline - $\quad$ Only Dr or MW can prevent & 4.19 & 1.54 \\
\hline - $\quad$ Must consult Dr or MW & 2.84 & 1.39 \\
\hline - $\quad$ Dr or MW should make decisions & 2.92 & 1.40 \\
\hline - If excellent care & 2.39 & 1.37 \\
\hline \multicolumn{3}{|l|}{ Pregnancy health motivation and effort } \\
\hline Pregnancy health effort and motivation*** & 6.08 & 0.70 \\
\hline Achievement motivation & 5.00 & 1.25 \\
\hline \multicolumn{3}{|l|}{ Approach/avoidance motivations } \\
\hline - $\quad$ Rather not know if anything is wrong & 1.62 & 1.28 \\
\hline - Desire to be involved in decisions & 6.59 & 0.76 \\
\hline Important to practice cultural beliefs & 4.70 & 1.24 \\
\hline Good to know sex of baby & 3.50 & 1.78 \\
\hline
\end{tabular}

**Item dropped from Fate sub-scale during EFA.

$* *$ This sub-scale did not meet criteria set in EFA and sub-scale items are now reported as stand alone items.

$* * *$ Care for sake of baby had highest mean of items

Table 5.47 shows significant associations between variables. Mastery, motivation and self efficacy for change have an association in a positive direction with good pregnancy 
nutrition effort and transition in regard to attachment toward the baby and looking forward to a new life stage.

Table 5.47. Transition Adaptation Adjusters:

\begin{tabular}{lll}
\hline Item/scale & Correlated with & R \\
\hline Information receipt interest & Sense of mastery & .374 \\
& Good nutrition effort) & .341 \\
& Self-efficacy for making health changes & .354 \\
Perceived positive information outcomes & Looking forward to a new life stage & .349 \\
& & .354 \\
Self-efficacy for making health changes & Information receipt interest & .468 \\
& Good nutrition effort level (pregnancy) & \\
Achievement motivation & & .306 \\
Pregnancy health effort and motivation & Maternal-fetal Attachment & .429 \\
& Pregnancy motivation and effort & .401 \\
Pregnancy motivation and effort & Achievement motivation & .429 \\
\hline
\end{tabular}

** All Pearson's correlations reported here significant at the .01 level (two tailed)

As shown in Table 5.48, 'Wanting the best for the baby' is a particularly high motivation for change. 'Time and pressure from commitments' was an important barrier affecting $43 \%$ ('impacts a little' to 'impacts a lot') of respondents in some way. Respondents were asked to specify if they had marked the 'other category'. The main reason was work.

Table 5 48. Influences for change to improve health in pregnancy

\begin{tabular}{lll}
\hline Item & Mean & SD \\
\hline Reasons for health changes made & & \\
Self-oriented & & \\
Feels physically better & 5.91 & 1.18 \\
Important to follow advice & 5.73 & 3.02 \\
Maximise own wellbeing & 5.72 & 1.40 \\
Will enjoy pregnancy more & 5.68 & 1.36 \\
Scared of becoming unwell & 5.19 & 1.71 \\
Pre-pregnancy changes * & 3.29 & 2.45 \\
Good reason to start & 2.76 & 2.37 \\
Felt pressured by others & 2.69 & 1.70 \\
Baby-oriented & & \\
Want the best for the baby's wellbeing & 6.83 & 0.54 \\
Scan, hearing heartbeat motivated & 6.01 & 1.41 \\
Something might happen to baby & 5.46 & 1.66 \\
\hline
\end{tabular}




\begin{tabular}{lll}
\hline Item & Mean & SD \\
\hline Enablers and barriers to change & & \\
Self-efficacy for making health changes & 5.79 & 0.96 \\
Other & 4.39 & 2.65 \\
Time & 3.79 & 1.93 \\
Feeling unwell & 3.33 & 1.82 \\
Sticking to changes & 2.92 & 1.75 \\
Lacking motivation & 2.88 & 1.84 \\
Money & 2.64 & 1.97 \\
Unsure how to & 2.27 & 1.47 \\
Transport & 1.89 & 1.50 \\
Poor support & 1.73 & 1.33 \\
Accommodation problems & 1.58 & 1.33 \\
\hline
\end{tabular}

*N/A box available for unplanned pregnancies

\subsubsection{Summary of Transition Adaptation Adjusters Descriptive Results}

Overall, women are highly motivated to care for their pregnancy and wish to be fully aware and involved in decisions regarding the pregnancy. Although self-interest is a major influence, concern for the baby's safety and wellbeing is significantly higher than any other variable, at the $95 \%$ confidence level. Information plays an important role for respondents as a means to adjust to the pregnancy. The importance of gaining knowledge is seen by the high attendance at antenatal classes also. Respondents rely heavily on medical and midwifery sources for information, although friends and family are also regarded highly. Although pregnancy is regarded as 'natural', women in this sample appear to have an affinity for technology, which does appear an oxymoron. The greatest barrier to achieving changes to improve health is time.

\subsubsection{THE TRANSITIONAL SELF DESCRIPTIVE RESULTS.}

This chapter sub-section presents descriptive results for the Transitional Self subdomain. Physical and psychosocial variables are included. The diagram below highlights the conceptual framework sub-domain discussed in this section.

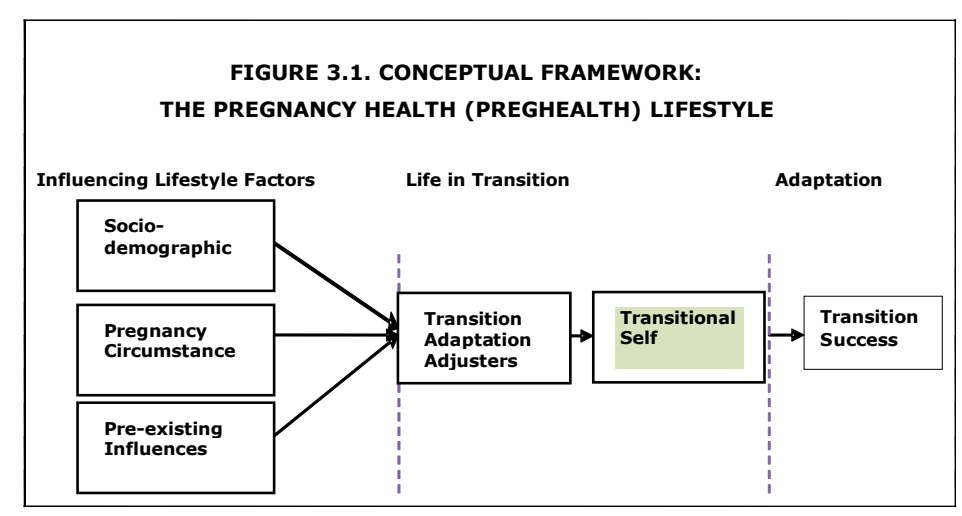




\section{PHYSICAL}

\subsubsection{Nutrition}

The nutrition construct variables are presented in table 5.49 to 5.52. Respondents indicate they make an effort to care for their nutrition, although a moderate degree of disruption occurs from morning sickness and change in liking/disliking for food. Tables 5.49 and 5.50 shows that the highest sample means were for avoidance behavioursavoiding 'more that 3 caffeine' and 'avoids risky food', although this does not reach to avoiding junk food (an item within the 'good nutrition' scale). Most women appear to make a substantial effort to eat well in pregnancy.

Table 5.49. Nutrition attitudes, Nutrition practice and Perceived barriers to good nutrition

\begin{tabular}{|c|c|c|c|}
\hline Variable & Sub-variable and item/scale & Mean & SD \\
\hline \multirow[t]{12}{*}{ Nutrition attitudes } & Good nutrition and outcome beliefs & & \\
\hline & Keeping nutrition advice will allow to stay well & 5.4 & 1.17 \\
\hline & Increases chances of a health baby & 5.38 & 1.22 \\
\hline & Reduces chances of a small baby & 4.58 & 1.34 \\
\hline & Eating pattern disturbance & & \\
\hline & Morning sickness interferes & 4.63 & 2.11 \\
\hline & Likes and dislikes changed & 4.16 & 1.99 \\
\hline & Developed cravings & 3.87 & 2.06 \\
\hline & Nutrition and weight gain & & \\
\hline & Too much gain and health problems after & 4.71 & 1.38 \\
\hline & If diet well-balanced don't mind weight & 4.35 & 1.74 \\
\hline & Need to take care about weight gain & 4.03 & 3.20 \\
\hline \multirow[t]{9}{*}{ Nutrition practices } & Good nutrition effort & & \\
\hline & Good nutrition effort & 5.07 & 1.09 \\
\hline & Intake against guidelines & & \\
\hline & Avoids more than 3 caffeine & 6.22 & 1.42 \\
\hline & Eats 2 fruits & 5.68 & 1.42 \\
\hline & Drinks 6 fluids & 5.58 & 1.54 \\
\hline & Eats 3 milk & 5.31 & 1.56 \\
\hline & Eats 3 vege serves & 5.06 & 1.46 \\
\hline & Low in fat, salt and sugar & 4.50 & 1.45 \\
\hline \multirow[t]{3}{*}{ Reducing nutrition risk } & Risky food avoidance & & \\
\hline & Avoids risky food & 6.30 & 1.22 \\
\hline & Folic acid intake (see Table 5.51 and 5.52) & & \\
\hline \multirow[t]{5}{*}{ Barriers to good nutrition } & Perceived barriers to good nutrition & & \\
\hline & Time pressures & 2.99 & 1.79 \\
\hline & Would like to care more & 2.91 & 1.71 \\
\hline & Hard to know how to eat healthily in pregnancy & 2.53 & 1.54 \\
\hline & Poor appetite & 2.52 & 1.76 \\
\hline
\end{tabular}




\begin{tabular}{llll}
\hline Variable & Sub-variable and item/scale & Mean & SD \\
\hline & Living situation & 2.08 & 1.65 \\
& Lack of money for food & 1.96 & 1.64 \\
No real interest in changing diet & 1.84 & 1.31 \\
Lack of skill in food preparation & 1.82 & 1.64 \\
Poor cooking facilities & 1.51 & 1.13 \\
Other** & 4.36 & 2.85 \\
\hline
\end{tabular}

$*$ Avoiding junk food has the lowest sample mean of all items

** 'Other' category most specified was work.

Table 5.50. Avoids risky foods and Intake against guidelines recommendations*

\begin{tabular}{|c|c|c|c|}
\hline Sub-variable /items/scale & Low (\%) & Mod (\%) & High (\%) \\
\hline \multicolumn{4}{|l|}{ Avoids risky foods } \\
\hline Avoids risky food & 5.3 & 9.0 & 85.4 \\
\hline \multicolumn{4}{|l|}{ Intake against guidelines } \\
\hline Avoids more than 3 caffeine & 8.6 & 9.0 & 82.0 \\
\hline Eats 2 fruits & 9.6 & 27.9 & 63.0 \\
\hline Drinks 6 fluids & 12.2 & 25.9 & 61.5 \\
\hline Eats 3 milk servings & 15.9 & 29.9 & 53.9 \\
\hline Ensures diet well balanced & 10.1 & 37.6 & 51.9 \\
\hline Eats 3 vegetable servings & 16.6 & 39.5 & 43.5 \\
\hline Low in fat, salt and sugar & 24.7 & 48.9 & 26.1 \\
\hline
\end{tabular}

*Daily food portions as given in MOH Guidelines for Pregnant Women (2006)

** Low ('none' to 'less than some of the time'), moderate ('some of the time' to 'more than some of the time') and high ('most often' or' all of the time'), categories reduced as given. 
A folic acid supplement was taken by $90.8 \%$ of women during the pregnancy. However, of the 434 women who took folic acid, only $47.3 \%$ (less than half) took it before becoming pregnant, which is the optimal time to have the supplement.

\section{Table 5 51. Folic acid intake*}

\begin{tabular}{lll}
\hline Item & $\mathbf{n}$ & $\mathbf{\%}$ \\
\hline Before pregnant & 226 & 47.3 \\
Once pregnancy confirmed & 208 & 43.5 \\
Did not take & 42 & 8.8 \\
Missing & 2 & 0.4 \\
Total & 478 & $100 \%$ \\
\hline
\end{tabular}

* Can be considered both a nutrition and risk reduction behaviour

Table 5.52 shows those who are in 'planned' pregnancy categories ('Wanted' and 'Wanted earlier" have a higher pre-pregnancy uptake of folic acid than others. (Note: Folic acid may also have been included in the vitamin supplement or prescribed medication categories by respondents).

Table 5.52. Timing of folic acid supplementation

\begin{tabular}{llllll}
\hline Timing & Wanted & $\begin{array}{l}\text { Wanted } \\
\text { earlier }\end{array}$ & $\begin{array}{l}\text { Not right } \\
\text { away }\end{array}$ & $\begin{array}{l}\text { Did not } \\
\text { want }\end{array}$ & Total \\
\hline Did not take & 10 & 2 & 23 & 7 & 42 \\
& $3.9 \%$ & $2.6 \%$ & $18.1 \%$ & $43.8 \%$ & $8.8 \%$ \\
Before pregnant & 161 & 55 & 10 & 0 & 226 \\
& $62.6 \%$ & $72.4 \%$ & $7.9 \%$ & $0.0 \%$ & $47.5 \%$ \\
Once pregnancy & 86 & 19 & 94 & 9 & 208 \\
confirmed & $33.5 \%$ & $25.0 \%$ & $74.0 \%$ & $56.3 \%$ & $43.7 \%$ \\
Total & 257 & 76 & 127 & 16 & 476 \\
& $100.0 \%$ & $100.0 \%$ & $100.0 \%$ & $100.0 \%$ & $100.0 \%$ \\
\hline
\end{tabular}

Fisher's Exact Test $p=.000$

\section{Vegetarianism}

It was noted that 29 respondents were in the 'yes' category for being vegetarian, $6.1 \%$ of the total sample.

\subsubsection{Exercise and Rest}

Table 5.53 shows that there are high means for attitudes toward benefits of exercise in pregnancy. The belief that exercise is dangerous in pregnancy has a relatively low mean. Negative internal barriers are generally stronger than external ones over exercise. High sample means were found for 'doesn't sleep as well' and 'needs to rest more'. 
Table 5.53. Exercise attitudes, Barriers and interference and Sleep and Rest

\begin{tabular}{|c|c|c|c|}
\hline Variable & Sub-variable and Item/scale & Mean & SD \\
\hline \multirow[t]{9}{*}{ Exercise attitudes } & Benefits & & \\
\hline & Regular exercise good for health & 6.09 & 0.95 \\
\hline & Helps in labour and birth & 5.86 & 1.14 \\
\hline & Helps relax and unwind & 4.99 & 1.54 \\
\hline & Controls weight & 5.17 & 2.00 \\
\hline & Danger for baby & & \\
\hline & Is dangerous for baby & 2.40 & 1.33 \\
\hline & Information sufficiency & & \\
\hline & Has right amount of information & 5.01 & 1.45 \\
\hline \multirow{12}{*}{$\begin{array}{l}\text { Barriers } \\
\text { exercise }\end{array}$} & Perceived interference scale & 3.56 & 1.68 \\
\hline & Internal barriers & & \\
\hline & Too tired or unwell & 3.89 & 1.78 \\
\hline & Difficult finding time & 3.77 & 1.86 \\
\hline & Activity uncomfortable & 3.33 & 1.80 \\
\hline & Confused about what to do & 2.77 & 1.67 \\
\hline & Never exercises anyway & 2.10 & 1.48 \\
\hline & External barriers & & \\
\hline & Work active so no need & 2.56 & 1.65 \\
\hline & People put pressure on to exercise & 2.39 & 1.75 \\
\hline & Living environment not conducive & 2.26 & 1.40 \\
\hline & Others discourage & 1.90 & 1.30 \\
\hline \multirow[t]{2}{*}{ Sleep and rest } & Needs to rest more & 6.19 & 1.17 \\
\hline & Doesn't sleep as well & 5.78 & 1.6 \\
\hline
\end{tabular}

By far the most common form of exercise undertaken was walking, followed by swimming or aquarobics. As in Table $5.54,81.2 \%$ did not have a planned exercise programme, but respondents were more likely to exercise more frequently if they had a planned exercise programme. For those respondents who had a plan, most relied on themselves as the plan designer. Frequency of exercise was cross-tabulated with gestational group and there was no significance found. This means that the rate of exercise is maintained throughout pregnancy despite increasing gestation and growth. 
Table 5.54. Exercise practices: Planning and Patterns

\begin{tabular}{|c|c|c|c|c|c|}
\hline Item & $\mathbf{n}$ & $\%$ & Item & $\mathbf{n}$ & $\%$ \\
\hline Planning & & & Patterns & & \\
\hline Has an exercise plan & & & Frequency of exercise* & & \\
\hline Yes & 86 & 18.0 & Low & 87 & 18.2 \\
\hline No & 388 & 81.2 & Moderate & 199 & 41.6 \\
\hline Missing & 4 & 0.8 & High & 189 & 39.5 \\
\hline Source of plan* $(n=86)$ & & & Missing & 3 & 0.6 \\
\hline Self & 51 & 60 & Activities * & & \\
\hline Book or pamphlet & 32 & 38.1 & Walking & 442 & 92.5 \\
\hline Gym or trainer & 27 & 32.1 & Swimming/Aquarobics & 121 & 25.3 \\
\hline Dr or Midwife & 18 & 21.4 & Yoga or meditation & 70 & 14.6 \\
\hline Physiotherapist & 5 & 5.9 & Pregnancy-programme & 39 & 8.2 \\
\hline \multirow[t]{6}{*}{ Other } & 7 & 8.3 & Weight training & 29 & 6.1 \\
\hline & & & Team sports & 17 & 3.6 \\
\hline & & & Aerobic classes & 11 & 2.3 \\
\hline & & & Other & 57 & 11.9 \\
\hline & & & & Mean & SD \\
\hline & & & Follows exercise recommendations & 4.66 & 1.46 \\
\hline
\end{tabular}

* Could answer as many options as relevant

Table 5 55. Frequency of exercise* and Has an exercise plan

\begin{tabular}{llll}
\hline Frequency group & No & Yes & Total \\
\hline Low & 84 & 2 & 86 \\
Moderate & $21.7 \%$ & $2.3 \%$ & $18.2 \%$ \\
& 167 & 31 & 198 \\
High & $43.2 \%$ & $36.0 \%$ & $41.9 \%$ \\
& 136 & 53 & 189 \\
Total & $35.1 \%$ & $61.6 \%$ & $40.0 \%$ \\
& 387 & 86 & 473 \\
& $100.0 \%$ & $100.0 \%$ & $100.0 \%$ \\
\hline
\end{tabular}

p. $=.000$

*Grouped as low, moderate and high.

\subsubsection{Substance Use}

As shown in Table 5.56, the mean for avoiding medication during pregnancy is high (84.9\% 'agree quite a lot' or 'strongly agree'). There is some variation with attitudes toward homeopathic remedies and 'over-the-counter' medication. Further profiling with cross tabulation shows a significant percentage of respondents believe that homeopathy is in someway beneficial, with relatively few respondents holding strong attitudes against them $(17.2 \%)$. There is a mixed response to taking OTC medication: $29.9 \%$ in disagreement, $33.9 \%$ more moderate, and $35.9 \%$ in agreement (a lot or strongly). There is a low declared rate of party pills/ marijuana. 
Table 5.56. Attitude to medication use in pregnancy and medication use

\begin{tabular}{lll}
\hline Item & Mean & SD \\
\hline Attitude & & \\
Avoid taking medication & 6.36 & 1.26 \\
Would take OTC meds & 4.51 & 1.91 \\
Homeopathic beneficial & 4.35 & 1.38 \\
Medication usage (n has taken)* & $\mathbf{n}$ & \\
Vitamin supplements & 371 & \\
Over the counter (OTC) & 277 & \\
Prescribed medication & 174 & \\
Homeopathic or herbal & 106 & \\
Marijuana & 12 & \\
Party pills & 6 & \\
\hline
\end{tabular}

*Could tick as many boxes as desired

\section{Tobacco use and Alcohol intake}

Tables 5.57 to 5.59 present results for 'Tobacco use' in pregnancy and 'Alcohol intake'. Attitudes against alcohol and smoking are strong.

In regard to smoking, there is slightly more tolerance toward others smoking around pregnant women than pregnant women themselves. In the 12 months preceding pregnancy, $26.9 \%$ of respondents were smoking. Of those respondents, $7.7 \%$ continue to smoke in the pregnancy. Almost all continuing smokers claim they have cut down, with most smoking less than five tobacco cigarettes per day.

Influences over choices regarding smokers (includes those who then ceased and those who continued) were highest for not wanting to harm the baby. By far the greatest influence over the decisions a respondent who smoked made in pregnancy regarding discontinuance or continuance is the wish not to harm the baby ( $95 \%$ confidence). It is not known whether the moderate only rate of influence by the midwife or doctor is that health professionals do not have a strong influence over tobacco cessation or reduction decisions, or, although less likely, there was no communication on the matter (and therefore it does not act as an influence).

Whilst the mean for 'don't want to damage health' and 'don't want to harm baby' is higher for those who cease over those who continue, it is not statistically significant at $95 \%$. One way ANOVA between time orientation and smoking profile showed significant difference at $95 \%$ confidence with non-smokers having a higher timer orientation mean (for planning ahead rather than living one day at a time) than for smokers (both ceased 
at pregnancy and continue to smoke). In regard to 'smoking profile' and 'Health as a value', although the mean goes down between non-smokers, ceased smoking and continues to smoke, there is no statistical difference at $95 \%$. There is some association between 'continuing to smoke' and 'continuing to drink alcohol' $(p<.013)$.

Table 5.57. Tobacco use

\begin{tabular}{lll}
\hline Item & Mean & SD \\
\hline Attitudes about smoking * & & \\
Pregnant women shouldn't smoke & 6.59 & 1.13 \\
Others shouldn't smoke around pregnant women & 5.93 & 1.54 \\
Smoking patterns $\mathbf{1 2}$ months before to onset & $\mathbf{n}$ & \\
Not smoking before & 349 & \\
Stopped smoking at pregnancy onset & 92 & \\
Continues to smoke & 37 & \\
Smoking change since pregnant**(n=129) & & \\
None now & 95 & \\
Cut down & 30 & \\
About the same & 3 & \\
Smoking more & 1 & \\
Influences over smoking choices** $(\mathbf{n}=\mathbf{1 2 9})$ & Mean & $\mathbf{S D}$ \\
Don't want to harm baby & 6.52 & 1.36 \\
Doesn't want to damage own health & 4.62 & 2.28 \\
Family and friends don't want me to smoke & 4.4 & 2.58 \\
Information in brochures and ads & 4.1 & 2.65 \\
Midwife or Dr suggested I stop & 3.31 & 2.81 \\
Helps reduce stress & 3.04 & 2.61 \\
If control number will be OK & 2.07 & 2.35 \\
\hline
\end{tabular}

*Answered by all respondents, **Answered by smoking at onset of pregnancy respondents

Table 5.58 shows there was a higher rate of smoking at the onset of pregnancy and continuance in Māori compared with non-Māori women.

Table 5.58. Smoking and pregnancy*: Māori vs Non Māori

\begin{tabular}{llll}
\hline Smoking profile & Non-Maori & Maori & Total \\
\hline Not smoking before & 324 & 24 & 348 \\
& $75.9 \%$ & $48.0 \%$ & $73.0 \%$ \\
Stopped at pregnancy & 79 & 13 & 92 \\
& $18.5 \%$ & $26.0 \%$ & $19.3 \%$ \\
Continues to smoke & 24 & 13 & 37 \\
& $5.6 \%$ & $26.0 \%$ & $7.8 \%$ \\
Total & 427 & 50 & 477 \\
& $100.0 \%$ & $100.0 \%$ & $100.0 \%$ \\
\hline p. $=.000$. & & &
\end{tabular}


Most respondents were not drinking alcohol in the pregnancy $(82.6 \%)$ as seen in Table 5. 59: Alcohol intake. A cross tabulation of alcohol intake in pregnancy by age group revealed that those in the 35-39 year age group have the greatest propensity to continue drinking in pregnancy. The 30-34 age group are the next largest group who continue to drink. ( $p=.000$, with 2 cells less than 5 .). By ethnicity, it appears to be British/European and 'other' ethnicities who are most likely to continue drinking.

Table 5.59. Alcohol intake

\begin{tabular}{lll}
\hline & & \\
\hline Attitude toward drinking in pregnancy & Mean & SD \\
Shouldn't have alcohol & 6.19 & 1.42 \\
Alcohol intake & $\mathbf{n}$ & $\%$ \\
Alcohol patterns since pregnant & & \\
Stopped altogether & 319 & 66.7 \\
Have cut down & 83 & 17.4 \\
Was not drinking & 76 & 15.9 \\
Total & & 100 \\
Frequency of alcohol consumption* & & \\
Never now & 314 & 65.7 \\
Monthly or less & 45 & 9.4 \\
2 to 4 times per month & 39 & 8.2 \\
2 to 3 times per week & 4 & 8.0 \\
Never even before pregnant & 76 & 15.9 \\
Total* & & \\
\hline
\end{tabular}

*There were a few respondents who said they had stopped drinking altogether but also nominated number of drinks, causing total not to add to $100 \%$. It is not known whether this is a genuine respondent error, or whether 'ceasing drinking' still allows for an amount of alcohol intake.

\subsubsection{Risk reduction}

\section{Wears safety belt}

The mean for wearing of safety belt at all times is 6.79 and $89.5 \%$ strongly agreed with the statement. This denotes a high degree of compliance (this is also a legal requirement in New Zealand).

\section{Care with teeth}

The mean is 4.91 (median 5 and SD 1.53), with $17.3 \%$ in the disagreement categories, $44.1 \%$ in 'neither agree' or 'disagree' or 'agree a little' and $37.9 \%$ are in 'agree quite a lot' or 'strongly agree'. This indicates that care with dental hygiene in pregnancy is not an extremely strongly held practice amongst some respondents. 
Note: Prenatal screening (scanning), has been previously reported under Pregnancy Circumstance, but could also be considered a pregnancy health risk reduction behaviour.

\section{PSYCHO-SOCIAL}

\subsubsection{Support}

Perceived support generally compares well with the importance of receiving support from that particular source (Table 5.60). Partners have the highest sample mean, but this is followed very closely by the LMC (no significant difference at $95 \%$ confidence). The mother, family friends and other family members are also important. For importance of partners support, 93.3\% are in 'rather and very important' categories, with $89.3 \%$ stating partner support is 'very important'. For importance of maternal support, $67.2 \%$ are in 'rather and very important' categories and $43.1 \%$ stating maternal support is very important.

Table 5.60. Support importance and Perceived support

\begin{tabular}{llllll}
\hline Item & Mean & SD & Item & Mean & SD \\
\hline $\begin{array}{l}\text { Source } \\
\text { importance* }\end{array}$ & & & $\begin{array}{l}\text { Perceived } \\
\text { support* }\end{array}$ & & \\
Partner & 6.60 & 1.50 & Partner & 6.19 & 1.66 \\
Mother & 5.64 & 1.82 & Mother & 5.68 & 1.86 \\
Family & 5.53 & 1.35 & Family & 5.70 & 1.42 \\
Friends & 5.59 & 1.24 & Friends & 5.81 & 1.31 \\
Midwife/Dr & 5.97 & 1.30 & Midwife/Dr & 6.03 & 1.17 \\
Internet & 1.23 & 1.70 & Internet & 1.10 & 1.86 \\
Other $\quad$ health & 3.50 & 2.65 & Other health & 2.76 & 2.81 \\
professionals & & & & & \\
\hline
\end{tabular}

*Option for father of baby if not partner not included in results as few respondents. N/A option also. Option for 'other" answered by a few respondents (not included in table). Mostly were work colleagues and were rated highly for perceived support.

Table 5.61 shows that all types of support are viewed as important and strongly desired. Pregnancy and birth-related support (that is, support concerned with direct provision for and interest in the development of the baby) have slightly higher means than those concerned with maternal health care.

Table 5.61. Type of support desired

\begin{tabular}{lll}
\hline Item & Mean & SD \\
\hline Pregnancy and birth-related & & \\
Showing interest in the development of the baby & 6.65 & 0.63 \\
Helping with preparation for baby's arrival & 6.36 & 0.9 \\
\hline
\end{tabular}




\begin{tabular}{lll}
\hline Item & Mean & SD \\
\hline Confidence about pregnancy management & 5.96 & 1.1 \\
Helping make pregnancy health decisions & 5.72 & 1.24 \\
Attending appointments and tests & 5.69 & 1.44 \\
Emotional and enjoyment & & \\
Making feel confident and secure about future & 6.57 & 2.9 \\
Talking about feelings & 6.17 & 1.08 \\
Providing physical affection & 6.01 & 1.3 \\
Keeping conflicts to a minimum & 5.75 & 1.43 \\
Helping have fun and a social life & 5.67 & 1.3 \\
Daily chores/financial & & \\
Helping with household chores & 5.74 & 1.34 \\
Providing financial support & 5.15 & 1.78 \\
\hline
\end{tabular}

\subsubsection{Coping}

Respondents found themselves generally 'more emotional' in pregnancy than usual. However, when 'solving problems', most respondents felt they could make rational decisions, if needed. Pearson's correlation 'Pregnancy state' anxiety and 'Life situation' anxiety were positively correlated at.345 (p. 000). This indicates that if a respondent felt anxious about their pregnant state, they might also feel anxious about other 'life' issues.

\section{Table 5.62. Coping, Anxiety, Emotions and reactions and Relaxation}

\begin{tabular}{lll}
\hline Item & Mean & SD \\
\hline Current emotional status & & \\
Pregnancy state anxiety & 4.28 & 1.24 \\
Life situation state anxiety & 3.39 & 1.38 \\
Problem solve rather than becoming emotional & 5.05 & 1.24 \\
More emotional now & 4.88 & 1.74 \\
Confident can give birth to a healthy baby & 5.93 & 1.04 \\
Stress/Relaxation activities* & $\mathbf{n}$ & $\%$ \\
Talking things over & 396 & 82.8 \\
TV or films & 384 & 80.3 \\
Reading & 367 & 76.8 \\
Going out with family/friends & 350 & 73.2 \\
Exercise & 261 & 55.2 \\
Antenatal touch & 127 & 26.6 \\
Meditation & 71 & 14.9 \\
Spiritual & 49 & 10.3 \\
Other** & 58 & $12.1 \%$ \\
\hline
\end{tabular}

* Could tick multiple options

**'Other' comprised of handcrafts and horse riding 


\subsubsection{Experiencing the Pregnant Body}

There is a high sample mean for confidence in ability to 'look good', without sacrificing comfort. There is a lower sample mean for actual pursuit of specific products and information. The mean for celebrity attraction (the degree to which respondents are attracted to celebrity news and lifestyles) is low amongst the respondent group. There was no significant difference between Body Satisfaction and 'Intendedness of pregnancy', except between 'Did not want' and 'Wanted,' 'Wanted earlier' categories, where the 'Did not want' has a significantly, lower mean.

Table 5.63. Pregnant body image, Appearance interest and Celebrity attraction

\begin{tabular}{lll}
\hline Item & Mean & SD \\
\hline Pregnancy body satisfaction & 5.59 & 1.19 \\
Weight worry & 3.55 & 1.38 \\
Celebrity attraction & 2.31 & 1.23 \\
Pregnancy looks/interest in appearance & & \\
Can look good & 5.81 & 1.33 \\
Comfort more important than fashion & 5.58 & 1.4 \\
Worry about stretch marks & 3.83 & 1.82 \\
Important others think look attractive & 3.77 & 1.66 \\
Reads information about looks & 3.11 & 1.74 \\
\hline
\end{tabular}

\section{Transitional self: Pearson's correlations}

As seen in Table 5.64, several Transitional Self variables are correlated with other variables from other conceptual sub-domains. In particular, 'Good nutrition effort', Prepregnancy health level' and information and motivation variables. Likewise, Exercise in pregnancy is positively associated with pre-pregnancy health and 'Good nutrition effort'. 'Pregnancy body satisfaction' is associated with several variables, and importantly, positively with those from the transit success sub-domain. 'Pregnancy body satisfaction is negatively correlated with 'Weight concerns'.

Table 5.64. Transitional self: Pearson's correlations*

\begin{tabular}{lll}
\hline Item/scale & with & $\mathbf{r}$ \\
\hline Good nutrition effort) & Pre-pregnancy self-reported health & .507 \\
& Environmentalism & .369 \\
& Information receipt interest & .341 \\
& Information processing and utilisation & .468 \\
& self efficacy & \\
& Pregnancy health effort and motivation & .401 \\
& & \\
Follows exercise recommendations & Good nutrition effort level (pregnancy) & .437 \\
& Pre-pregnancy health level & .373 \\
\hline
\end{tabular}




\begin{tabular}{|c|c|c|}
\hline Item/scale & with & $\mathbf{r}$ \\
\hline \multirow{3}{*}{$\begin{array}{l}\text { Pregnancy. health \& outcomes state } \\
\text { anxiety }\end{array}$} & Trait anxiety & .339 \\
\hline & Dimensions of wellness & -.322 \\
\hline & Weight concerns & .306 \\
\hline \multirow[t]{6}{*}{ Pregnancy body satisfaction } & Maternal/fetal attachment & .611 \\
\hline & Weight concerns & -.466 \\
\hline & Dimensions of wellness & .406 \\
\hline & Rating as a life event & .419 \\
\hline & Looking forward to a new life stage & .450 \\
\hline & Happy to leave childless days behind & .365 \\
\hline \multirow[t]{4}{*}{ Weight concerns } & Concern for physical appearance & .300 \\
\hline & $\begin{array}{l}\text { Pregnancy health \& outcomes state } \\
\text { anxiety }\end{array}$ & .306 \\
\hline & Celebrity attraction & .344 \\
\hline & Pregnancy body satisfaction & -.466 \\
\hline \multirow[t]{2}{*}{ Celebrity attraction } & Pregnancy weight concerns & .344 \\
\hline & Concern for physical appearance & .443 \\
\hline
\end{tabular}

* All Pearson's correlations reported here significant at the.$\leq 01$ level (two tailed)

\subsubsection{Satisfaction with Living Arrangements}

Table 5.65 shows that $90.2 \%$ are happy with their present accommodation. The largest accommodation problems are transport, warmth and comfort and feeling the place is not suitable for a baby. Two respondents only checked the fear of violence item. It is not known if there was an inhibition response or another reason for a lower than expected response and is substantially less than current rates of domestic violence reported for pregnancy.

\section{Table 5.65. Satisfaction with living arrangements}

\begin{tabular}{lll}
\hline Item & $\mathbf{n}$ & $\mathbf{\%}$ \\
\hline Happy with present accommodation and living situation & 431 & 90.2 \\
Inadequate transport & 33 & 6.9 \\
Place or situation not well suited for a baby & 33 & 6.9 \\
Warmth and comfort not satisfactory & 28 & 5.9 \\
Too many people & 22 & 4.6 \\
Don't get on with others am living with & 3 & 0.6 \\
Concerned for personal safety (violence) & 2 & 0.4 \\
\hline
\end{tabular}

*Respondents could tick multiple options 


\subsubsection{Summary of the Transitional Self descriptive results}

Overall, women made a substantial effort to eat well in pregnancy and avoid 'risky' food. Folic acid intake was high, but timing of intake was mixed. Most respondents were moderate exercisers with walking the most common form of exercise. Alcohol and tobacco cessation was met with a high level of compliance for most women. The safety of the baby was the highest driver of substance avoidance. Respondents desired a high level of emotional support, but their needs were generally met well by their important others and LMC. A number of relaxation activities were engaged in. Overall, respondents felt a high degree of body satisfaction. A positive response to the experience of the pregnant body was associated with other positive expressions of transition.

\subsubsection{TRANSITION SUCCESS DESCRIPTIVE RESULTS}

This section presents results of analysis of the final dimension of the conceptual framework. It represents the variables used to demonstrate a success transition journey within the pregnancy course. The sub-domain in focus is highlighted in the conceptual framework diagram following.

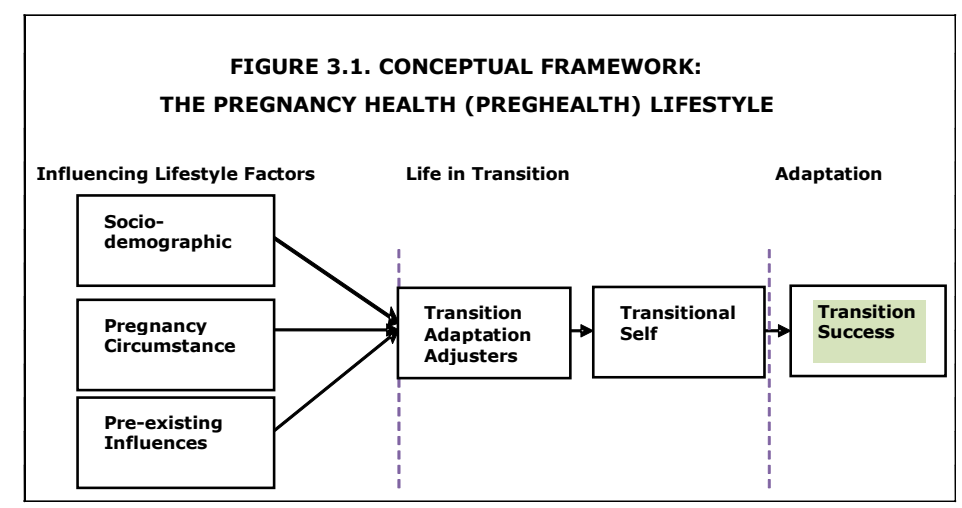




\subsubsection{Wellbeing, Relationship with Baby and Orientation to the Future}

The results show that respondents were generally feeling well. However some interesting specific insights emerged. For instance, $56.5 \%$ of respondents feel tired 'more than some of the time' and there was a low sample mean for 'feeling better than usual', meaning that pregnant women tend not to feel 'better' than they do when notpregnant. Maternal-fetal attachment score is high. Although respondents are looking forward to a new life stage, there are some respondents that are reticent about leaving their childless days behind.

An ANOVA test showed that there was no significant difference between 'wanted' pregnancies and 'mistimed' pregnancies in regard to attachment, meaning that planned and unplanned pregnancies formed the same level of attachment in this sample group. Those with pregnancies reported as 'did not want' have a significantly lower level of attachment that the three other categories of 'Intendedness' $(p<.001) . \quad$ Likewise, comparison with 'Perceptions of wellness' and 'Intendedness' showed that 'did not want' respondents reported significantly lower means for feeling well than other categories of 'Intendedness' $(p<0.01)$.

Table 5.66. Wellbeing, Attachment and Orientation to the future

\begin{tabular}{lll}
\hline Item/scale & Mean & SD \\
\hline Wellbeing & 5.27 & 0.93 \\
Perception of wellness & 4.96 & 1.80 \\
Feeling tired & 2.89 & 1.57 \\
$\begin{array}{l}\text { Better than usual } \\
\text { Relationship with Baby }\end{array}$ & \\
$\begin{array}{l}\text { Maternal-fetal attachment } \\
\text { Orientation to Future }\end{array}$ & 6.29 & 0.83 \\
$\begin{array}{l}\text { Happy to leave childless days behind } \\
\text { Looking forward to a new life stage }\end{array}$ & 4.99 & 1.55 \\
\hline
\end{tabular}

The Transition Success sub-domain variables have several associations with other variable both within their own sub-domain and others (Table 5.67). 'Maternal/fetal attachment' is strongly positively correlated with 'Pregnancy body satisfaction' and 'looking forward' to a new life stage. 'Pregnancy state anxiety' and 'weight concerns' are negatively correlated to perception of wellness. 
Table 5.67. Transition success: Pearson's correlations

\begin{tabular}{|c|c|c|}
\hline Item/scale & Correlated with & $\mathbf{r}$ \\
\hline \multirow[t]{5}{*}{ Maternal/fetal attachment } & Pre-pregnancy health+ & .384 \\
\hline & Pregnancy body satisfaction & $.611^{*}$ \\
\hline & Importance of accomplishing goals & .306 \\
\hline & Looking forward to a new life stage & .600 \\
\hline & Happy to leave childless days behind & .425 \\
\hline \multirow[t]{6}{*}{ Perceptions of wellness } & Pregnancy weight concerns & -.322 \\
\hline & Pre-pregnancy health & .387 \\
\hline & Pregnancy body satisfaction & .406 \\
\hline & Rating as a life event & .326 \\
\hline & Pregnancy state anxiety & -.322 \\
\hline & Sense of mastery & .380 \\
\hline \multirow[t]{5}{*}{ Looking forward to a new life stage } & Maternal/fetal attachment & .600 \\
\hline & Pregnancy body satisfaction & .450 \\
\hline & Rating as a life event & .420 \\
\hline & Happy to leave childless days behind & .523 \\
\hline & Information outcome beliefs & .349 \\
\hline \multirow[t]{4}{*}{ Happy to leave childless days behind } & Maternal/fetal attachment & .425 \\
\hline & Pregnancy body satisfaction & .365 \\
\hline & Rating as a life event & .354 \\
\hline & Looking forward to a new life stage & .523 \\
\hline
\end{tabular}

** All Pearson's correlations reported here significant at the .001 level (two tailed)

\subsubsection{Summary of Transition Success results}

Overall, respondents generally had a feeling of wellbeing, although they report feeling tired and not feeling better than they would normally. They felt strongly attached to their babies and were looking forward to a new life stage. Positive attachment to their baby was also associated with positive feelings about their pregnant body and moving forward to a new life stage.

\subsubsection{DESCRIPTIVE RESULTS SUMMARY}

The descriptive results have set the scene for the next stage of the analysis. The data was analysed and aggregated in a way that describes the range of attitudes and behaviours of women that make up the health lifestyles of this sample of women. It has been shown that while many variables are similar within the women, there are those that point to variation and difference for some groups. The next stage of the analysis will lead to identification of segments with distinct characteristics. 


\subsection{CLUSTER ANALYSIS}

The final component of the data analysis was to segment women into pregnancy health lifestyle groups. This was undertaken in order to identify homogeneous pregnancy health lifestyle segments according to the conceptual dimension variables clustered. The sub-cluster stage also served as a significant data reduction phase. For all clustering procedures, a 'Two-Step Clustering' technique was used (a complete discussion of this process was presented in Chapter 4 ). The clustering procedure was divided into five stages that include; determining variables for inclusion in sub-clusters and for profiling, forming and analysing the sub clusters, selecting variables for the Gravidographic clusters through data reduction and then finally forming and profiling the Gravidographic clusters. Selection of variables for the final Gravidographic segmentation was based on the sub-cluster results, conceptual significance and that they were regarded as salient from the descriptive analysis. Once the Gravidographic clusters had been formed they were profiled against variables that had been withheld from clustering procedures and retained for this purpose. Figure 4.1 has been repeated here to refresh the reader about the stages of the clustering process.

\subsubsection{STAGES OF CLUSTER PROCEDURE}

Results for the stages are presented in this section. Stages 1 and 2 are combined in the presentation of results for clarity and flow.

Stage 1 and 2 assisted in data reduction and also provided an opportunity to assess and better understand how variables clustered within sub-domains and which variables appeared most distinctive as driving sub-cluster formation. Each sub-cluster consisted of the selection of variables from a sub-domain or a

Figure xxxx: Clustering procedure leading to identification of Gravidographic segments

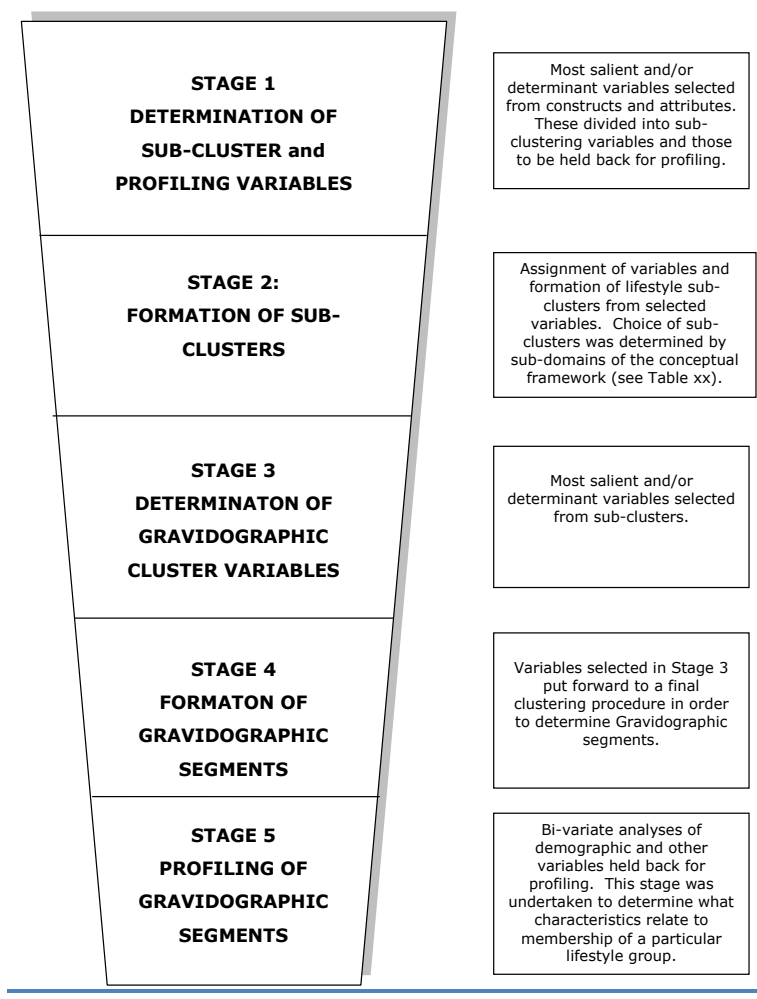
collective of variables (for instance, nutrition and exercise) considered sufficiently important to examine closely due to quantity of contributing variables and/or complexity of the constructs that variables belonged to. This was determined primarily on conceptual salience and researcher judgement. Sub-domains within the Pre-exiting Lifestyle Factors dimension were not sub-clustered. This is because they were held back to be used for the profiling as they are considered stable and enduring characteristics. The one exception to this is the Feelings about the Pregnancy construct, which is added 
at the Gravidographic cluster stage. The rationale for this will be discussed at a later stage. Profiling variables were selected according to their appropriateness to describe and add meaning to the derived Gravidographic clusters. Stage 2, the sub-clustering stage, allowed for the removal of variables that were not distinctive, that is, did not vary significantly in regard to means across derived sub-clusters as advocated by Hair (2010). This action meant that variables put forward to the Gravidographic stage exhibited maximum difference between objects. Any exceptions to this were that a variable (for example, 'Wears a safety belt') that although showing little variation, was considered conceptually critical to the holistic framework of pregnancy health lifestyle and therefore went forward.

The sub-clusters are presented in the sub-section following. Results provided for each sub-cluster include:

- Scale and item mapping to variables and constructs

- Sub-cluster distribution

- Sub-cluster categorical measures

- Sub-cluster continuous measures (sub-cluster means and 95\% confidence statistical difference provided)

- Sub-cluster interpretation

- Sub-cluster items /scales put forward to the Gravidographic stage.

Items and scales are referred to by their working titles or some sub-clusters, where a table describing the sub-cluster groupings (within a sub-cluster) is thought to be useful, this is also provided along with the text within the sub-cluster interpretation section.

\subsubsection{Information sub-cluster (Transition Adjusters sub-domain) Scale and item mapping to variables and constructs}

The constructs and variables selected for this sub-cluster contained all of the Information variables in the Transition Adjusters sub-domain, apart from the variables, Antenatal classes and Arranging pregnancy care. As by far most women had attended Antenatal classes, it was considered to not be distinguishing and therefore of more use to assess decisions made by women (eg., type of classes attended) at the profiling stage. For Information source usefulness, six of the 25 possible source usefulness items were selected (these were those rated the most highly in the descriptive results. 
Table 5.68. Information sub-cluster scale and item mapping to variables and constructs

\begin{tabular}{|c|c|c|}
\hline Constructs/attribute & Variable & $\begin{array}{l}\text { Scale/item by working } \\
\text { title }\end{array}$ \\
\hline \multirow[t]{2}{*}{ Information Acquisition } & Information seeking activity & $\begin{array}{l}\text { Information receipt interest } \\
\text { Ways of knowing } \\
\text { - } \quad \text { Life experience } \\
\text { - Medical is best } \\
\text { - } \quad \begin{array}{l}\text { Need to balance } \\
\text { with intuition }\end{array}\end{array}$ \\
\hline & Information sources and usefulness & $\begin{array}{ll}\text { Source usefulness } \\
\text { - } & \text { Partner } \\
\text { - } & \text { Mother } \\
\text { - } & \text { Alternate therapists } \\
\text { - } & \text { Midwife or doctor } \\
\text { - } & \text { Medical tests } \\
\text { - } & \text { Internet search }\end{array}$ \\
\hline \multirow{5}{*}{$\begin{array}{l}\text { Information Processing and } \\
\text { utilisation }\end{array}$} & Information processing self efficacy & Confidence in decisions \\
\hline & Perceived information benefits and outcomes & $\begin{array}{l}\text { Perceived positive } \\
\text { information outcomes }\end{array}$ \\
\hline & Difficulties and barriers processing information & $\begin{array}{l}\text { Too much a bad thing } \\
\text { Gets conflicting information }\end{array}$ \\
\hline & Resistance to change & Means too many restrictions \\
\hline & Changes as a result of information & Information change result \\
\hline
\end{tabular}

Table 5.69. Information Sub-cluster distribution

\begin{tabular}{llll}
\hline Cluster & $\mathbf{N}$ & \% of Combined & \% of Total \\
\hline 1 & 217 & $47.4 \%$ & $45.4 \%$ \\
2 & 153 & $33.4 \%$ & $32.0 \%$ \\
3 & 88 & $19.2 \%$ & $18.4 \%$ \\
Combined & 458 & $100.0 \%$ & $95.8 \%$ \\
Excluded & 20 & & $4.2 \%$ \\
Total & 478 & & $100.0 \%$ \\
\hline
\end{tabular}

The cluster number was fixed at 3. $\mathrm{C} 1$ is the largest cluster containing almost half of the respondents. $\mathrm{C} 2$ is the next largest, followed by $\mathrm{C} 3$.

\section{Information Sub-cluster: Categorical Measures}

There are no categorical measures in this sub-cluster 
Table 5.70. Information Sub-cluster: Continuous Measures

\begin{tabular}{|c|c|c|c|c|c|}
\hline \multirow[t]{2}{*}{ Item/scale } & \multicolumn{2}{|r|}{ Cluster } & \multicolumn{2}{|l|}{ Means } & \multirow{2}{*}{$\begin{array}{l}\text { Information SC Continuous measures: } 95 \% \text { Confidence } \\
\text { Intervals }\end{array}$} \\
\hline & $\mathbf{1}$ & 2 & 3 & Combined & \\
\hline \multicolumn{6}{|l|}{ Information receipt interest } \\
\hline Information receipt interest & 6.50 & 5.48 & 5.77 & 6.02 & $\begin{array}{l}\mathrm{C} 2 \text { and } \mathrm{C} 3 \text { are below the overall mean. There is no significant } \\
\text { difference between } \mathrm{C} 2 \text { and } \mathrm{C} 3 . \mathrm{C} 1 \text { is significantly different from } \\
\text { the two other clusters. }\end{array}$ \\
\hline \multicolumn{6}{|l|}{ Ways of knowing } \\
\hline Gets from life experience & 4.67 & 3.92 & 4.57 & 4.40 & $\begin{array}{l}\mathrm{C} 2 \text { is below the overall mean and significantly different from } \mathrm{C} 1 \\
\text { and } \mathrm{C} 2 \text {. There is no significant difference between } \mathrm{C} 1 \text { and } \mathrm{C} 3 \text {. }\end{array}$ \\
\hline Medical is best & 3.81 & 3.42 & 3.53 & 3.62 & $\begin{array}{l}\mathrm{C} 2 \text { and } \mathrm{C} 3 \text { are below the overall mean. There is no significant } \\
\text { difference between the clusters. }\end{array}$ \\
\hline Need to balance with intuition & 5.75 & 5.66 & 5.40 & 5.65 & $\begin{array}{l}\text { C3 is below the overall mean. However, there is no significant } \\
\text { difference between the clusters. }\end{array}$ \\
\hline \multicolumn{6}{|l|}{ Source usefulness } \\
\hline Partner & 3.78 & 4.13 & 3.86 & 3.91 & $\begin{array}{l}\mathrm{C} 1 \text { and } \mathrm{C} 3 \text { are slightly below the overall mean and } \mathrm{C} 2 \text { above. } \\
\text { However, there is no significant difference between the clusters. }\end{array}$ \\
\hline Mother & 4.63 & 5.14 & 4.44 & 4.76 & $\begin{array}{l}\mathrm{C} 1 \text { and } \mathrm{C} 3 \text { are below the overall mean. There is no significant } \\
\text { difference between the clusters. }\end{array}$ \\
\hline Alternate therapists & 2.07 & 2.07 & 2.82 & 2.21 & $\begin{array}{l}\mathrm{C} 1 \text { and } \mathrm{C} 2 \text { are below the overall mean and } \mathrm{C} 3 \text { above. There is no } \\
\text { significant difference between the clusters. }\end{array}$ \\
\hline Midwife or doctor & 6.88 & 6.81 & 5.61 & 6.61 & $\begin{array}{l}\text { C3 is below the mean and } \mathrm{C} 1 \text { and } \mathrm{C} 2 \text { above. } \mathrm{C} 3 \text { is significantly } \\
\text { different from } \mathrm{C} 2 \text { and } \mathrm{C} 3 \text {. }\end{array}$ \\
\hline Medical tests & 6.76 & 6.74 & 5.20 & 6.45 & $\begin{array}{l}\mathrm{C} 3 \text { is below the mean and } \mathrm{C} 1 \text { and } \mathrm{C} 2 \text { above. } \mathrm{C} 3 \text { is significantly } \\
\text { different from } \mathrm{C} 2 \text { and } \mathrm{C} 3 \text {. }\end{array}$ \\
\hline Internet search & 6.09 & 3.25 & 4.89 & 4.91 & $\begin{array}{l}\mathrm{C} 1 \text { is above the mean, } \mathrm{C} 2 \text { below and } \mathrm{C} 3 \text { on the mean. Each } \\
\text { cluster is significantly different from the other. }\end{array}$ \\
\hline
\end{tabular}




\begin{tabular}{|c|c|c|c|c|c|}
\hline Item/scale & & Cluster & Means & & $\begin{array}{l}\text { Information SC Continuous measures: } 95 \% \text { Confidence } \\
\text { Intervals }\end{array}$ \\
\hline \multicolumn{6}{|l|}{ Perceived difficulties and barriers } \\
\hline \multicolumn{6}{|l|}{ processing information } \\
\hline Too much is a bad thing & 3.54 & 4.09 & 3.97 & 3.81 & $\begin{array}{l}\mathrm{C} 1 \text { is below the overall mean. However, there is no significant } \\
\text { difference between the clusters. }\end{array}$ \\
\hline Gets conflicting information & 4.39 & 4.57 & 4.18 & 4.41 & $\begin{array}{l}\mathrm{C} 3 \text { is below the overall mean. However, there is no significant } \\
\text { difference between the clusters. }\end{array}$ \\
\hline \multicolumn{6}{|l|}{ Resistance to change } \\
\hline Means too many restrictions & 1.67 & 2.36 & 2.66 & 2.09 & $\begin{array}{l}\text { C1 is below the overall mean and significantly different from C2 } \\
\text { and C3. However, there is no significant difference between C2 } \\
\text { and } 3 .\end{array}$ \\
\hline $\begin{array}{l}\text { Perceived positive information } \\
\text { outcomes }\end{array}$ & & & & & \\
\hline Perceived positive information outcomes & 6.17 & 5.5 & 5.44 & 5.81 & $\begin{array}{l}\mathrm{C} 2 \text { and } \mathrm{C} 3 \text { are below the overall mean and } \mathrm{C} 1 \text { above. } \mathrm{C} 1 \text { is } \\
\text { significantly different from } \mathrm{C} 2 \text { and } \mathrm{C} 3 \text {. }\end{array}$ \\
\hline \multicolumn{6}{|l|}{$\begin{array}{l}\text { Information processing and } \\
\text { utilisation self-efficacy }\end{array}$} \\
\hline Confidence in decisions & 6.22 & 5.32 & 5.60 & 5.80 & $\begin{array}{l}\mathrm{C} 2 \text { and } \mathrm{C} 3 \text { are below the overall mean. There is no significant } \\
\text { difference between } \mathrm{C} 2 \text { and } \mathrm{C} 3 . \mathrm{C} 1 \text { is significantly different from } \\
\text { the two other clusters. }\end{array}$ \\
\hline \multicolumn{6}{|l|}{ Change as a result of information } \\
\hline Positive` behaviour response & 5.65 & 5.13 & 5.11 & 5.37 & $\begin{array}{l}\mathrm{C} 2 \text { and } \mathrm{C} 3 \text { are below the overall mean. There is no significant } \\
\text { difference between } \mathrm{C} 2 \text { and } \mathrm{C} 3 . \mathrm{C} 1 \text { is significantly different from } \\
\text { the two other clusters. }\end{array}$ \\
\hline
\end{tabular}




\section{Information sub-cluster interpretation}

For many of the variables, there is no significant difference between clusters. Where significance difference was found, it was between $\mathrm{C} 1$ and $\mathrm{C} 2 / \mathrm{C} 3$, which tended to be similar. As seen in the descriptive results, there are high means for information search and interest. All clusters value information and change as a result. Cluster 1 is the most mobilised to receive and respond to information and has the highest Internet use.

\section{Contributing variables to Gravidographic cluster stage}

The two variable items below were selected to go forward to the Gravidographic clustering as there was some statistical significance between clusters (Cluster 1 is statistically different from $\mathrm{C} 2$ and $\mathrm{C} 3$ ). These items relate to interest in receiving information and the positive benefits perceived from information utilisation.

Table 5.71. Information sub cluster items/scales put forward to Gravidographic cluster stage

\begin{tabular}{lll}
\hline Construct/attribute & Variable & Item/scale \\
\hline Information acquisition & Information receipt interest & Information receipt interest \\
Information processing & Perceived information benefits and & Perceived positive \\
and utilisation & outcomes & information outcomes \\
\hline
\end{tabular}

\subsubsection{Other Attitudes and Drivers sub-cluster (Transition Adjusters sub- domain)}

\section{Scale and item mapping to variables and constructs}

In this sub-cluster, the variables concerned with attitudes or resources that influenced respondents' choices regarding pregnancy health were clustered. 
Table 5.72. Other Attitudes and Drivers sub-cluster Scale and item mapping to variables and constructs

\begin{tabular}{|c|c|c|}
\hline Construct & Variable & Scale/item by working title \\
\hline \multirow{10}{*}{$\begin{array}{l}\text { Affinity for } \\
\text { technology in } \\
\text { pregnancy }\end{array}$} & Affinity for technology in pregnancy & Pregnancy is a natural process- \\
\hline & & Should trust nature \\
\hline & & Need a Doctor as well as M/W \\
\hline & & Need a scan in pregnancy \\
\hline & & Technology makes birth safer \\
\hline & Internal Health Locus of Control & Internal HLOC \\
\hline & $\begin{array}{l}\text { Powerful others: External health locus } \\
\text { of Control }\end{array}$ & ELOC PO If excellent care \\
\hline & & $\begin{array}{l}\text { ELOC PO Only DR or midwife can } \\
\text { prevent }\end{array}$ \\
\hline & & ELOC PO Must consult Dr or midwife \\
\hline & Fate: External Health Locus of Control & HLOC-Fate \\
\hline $\begin{array}{l}\text { Enablers and } \\
\text { barriers }\end{array}$ & Self-efficacy for making health changes & Self-efficacy for health changes \\
\hline \multirow{3}{*}{$\begin{array}{l}\text { Motivation/involve } \\
\text { ment and effort }\end{array}$} & Pregnancy health effort and motivation & Pregnancy health effort and motivation \\
\hline & Approach/avoidance & Rather not know if something wrong \\
\hline & Goal accomplishment & Importance of accomplishing goals \\
\hline
\end{tabular}

Table 5.73. Other Attitudes and Drivers sub-cluster distribution

\begin{tabular}{lccc}
\hline Cluster & $\mathbf{N}$ & \% Combined & \% of Total \\
\hline 1 & 148 & $31.5 \%$ & $31.0 \%$ \\
2 & 111 & $23.6 \%$ & $23.2 \%$ \\
3 & 211 & $44.9 \%$ & $44.1 \%$ \\
Combined & 470 & $100.0 \%$ & $98.3 \%$ \\
Excluded & 8 & & $1.7 \%$ \\
Total & 478 & & $100.0 \%$ \\
\hline
\end{tabular}

Cluster 3 is the largest cluster. Cluster 2 is the smallest, almost half the size of Cluster 3. Cluster 1 could be considered approximately midway in size between the two other clusters.

\section{Sub-cluster Categorical Measures}

There are no categorical measures in this sub-cluster. 
Table 5.74. Other Attitudes and Drivers sub-cluster continuous measures

\begin{tabular}{|c|c|c|c|c|c|}
\hline \multirow[t]{2}{*}{ Item/Scale } & \multicolumn{4}{|c|}{ Cluster Means } & \multirow[t]{2}{*}{ Information SC Continuous measures: $95 \%$ Confidence Intervals } \\
\hline & $\mathbf{1}$ & 2 & 3 & Combined & \\
\hline \multicolumn{6}{|l|}{$\begin{array}{l}\text { Affinity for technology in } \\
\text { pregnancy }\end{array}$} \\
\hline Is a natural process- & 5.87 & 6.41 & 5.30 & 5.74 & $\mathrm{C} 1$ and $\mathrm{C} 2$ are above the mean. $\mathrm{C} 3$ is below the mean. None of the clusters overlap. \\
\hline $\begin{array}{l}\text { Should trust nature rather than } \\
\text { rely on technology. }\end{array}$ & 3.38 & 4.28 & 2.75 & 3.31 & $\mathrm{C} 1$ and $\mathrm{C} 2$ are above the mean. $\mathrm{C} 3$ is below the mean. None of the clusters overlap. \\
\hline Need a Doctor as well as M/W & 4.51 & 2.87 & 3.65 & 3.74 & $\begin{array}{l}\mathrm{C} 1 \text { is above the mean. } \mathrm{C} 2 \text { is below the mean and } \mathrm{C} 3 \text { on at them mean. None of the } \\
\text { clusters overlap. }\end{array}$ \\
\hline Need a scan in pregnancy & 6.24 & 3.77 & 5.22 & 5.20 & $\begin{array}{l}\mathrm{C} 1 \text { is above the mean. } \mathrm{C} 2 \text { is below the mean and } \mathrm{C} 3 \text { on at them mean. None of the } \\
\text { clusters overlap }\end{array}$ \\
\hline Technology makes birth safer & 6.16 & 4.77 & 5.86 & 5.70 & $\begin{array}{l}\text { C1 and C3 are above the mean and overlap slightly. C2 is below the mean and does not } \\
\text { overlap with any other cluster. }\end{array}$ \\
\hline \multicolumn{6}{|l|}{ Internal HLOC } \\
\hline Internal HLOC & 5.20 & 4.57 & 4.31 & 4.65 & $\begin{array}{l}\mathrm{C} 1 \text { is above the mean and does not overlap with any other clusters. } \mathrm{C} 2 \text { and } 3 \text { are below } \\
\text { the mean and overlap. }\end{array}$ \\
\hline \multicolumn{6}{|l|}{$\begin{array}{l}\text { External HLOC: Powerful } \\
\text { others }\end{array}$} \\
\hline $\begin{array}{l}\text { If excellent care will not get } \\
\text { sick }\end{array}$ & 3.01 & 1.95 & 2.15 & 2.37 & $\begin{array}{l}\mathrm{C} 1 \text { is above the mean and does not overlap with any other clusters. } \mathrm{C} 2 \text { and } 3 \text { are below } \\
\text { the mean and overlap. }\end{array}$ \\
\hline Only Dr or MW can prevent & 4.58 & 3.82 & 4.13 & 4.20 & $\mathrm{C} 1$ is above the mean and overlaps with $\mathrm{C} 3 . \mathrm{C} 2$ and 3 are below the mean and overlap. \\
\hline Must consult Dr or MW & 3.22 & 2.34 & 2.84 & 2.84 & $\begin{array}{l}\mathrm{C} 1 \text { is above the mean, } \mathrm{C} 3 \text { on the mean and } \mathrm{C} 2 \text { below. } \mathrm{C} 1 \text { and } \mathrm{C} 2 \text { do not overlap. } \mathrm{C} 1 \\
\text { and } \mathrm{C} 3 \text { overlap, as do } \mathrm{C} 2 \text { and } \mathrm{C} 3 \text {. }\end{array}$ \\
\hline \multicolumn{6}{|l|}{ External HLOC: Fate } \\
\hline HLOC-Fate & 3.97 & 3.46 & 3.87 & 3.81 & $\begin{array}{l}\mathrm{C} 1 \text { and } \mathrm{C} 3 \text { are above the mean, } \mathrm{C} 2 \text { below. } \mathrm{C} 1 \text { and } \mathrm{C} 2 \text { do not overlap. } \mathrm{C} 1 \text { and } \mathrm{C} 3 \\
\text { overlap, as do } \mathrm{C} 2 \text { and } \mathrm{C} 3 \text {. }\end{array}$ \\
\hline \multicolumn{6}{|l|}{$\begin{array}{l}\text { Self-efficacy for making } \\
\text { health changes }\end{array}$} \\
\hline Self-efficacy for health changes & 5.84 & 6.10 & 5.59 & 5.79 & $\mathrm{C} 1$ and $\mathrm{C} 2$ are above the mean, $\mathrm{C} 3$ below. $\mathrm{C} 2$ and $\mathrm{C} 3$ do not overlap. $\mathrm{C} 1$ overlaps with \\
\hline
\end{tabular}


Item/Scale

Cluster Means

Information SC Continuous measures: 95\% Confidence Intervals

Pregnancy health

motivation and effort

Health motivation and effort

6.53

6.20

5.69

Approach/avoidance

Rather not know if something

1.18

2.61

1.38

wrong

Goal accomplishment

Importance of accomplishing goals
C2 and C3.

6.08 $\mathrm{C} 1$ and $\mathrm{C} 2$ are over the mean, $\mathrm{C} 3$ below. None of the clusters overlap.

1.61 C1 and C3 are below the mean and overlap. C2 is above the mean and does not overlap with any other cluster.

5.00 C1 is above the mean, C2 and C3 below. None of the clusters overlap. 


\section{Other Attitudes and Drivers sub-cluster interpretation}

There is statistical difference in the sub-cluster results for many of the variables. The most significant difference between the sub-clusters is in the 'Affinity for technology' items.

Table 5.75. Other Attitudes and Drivers sub-cluster interpretation

\begin{tabular}{ll}
\hline Cluster & Interpretation \\
\hline $\mathbf{1}$ & Cluster 1 has the highest means for being supportive of technology. Respondents have the highest \\
& mean for HLOC, but also for Powerful Others Health Locus of Control. C1 has the highest mean for \\
& accomplishing goals. \\
$\mathbf{2}$ & Cluster 2 is the cluster least inclined toward technology and significantly different on all the \\
& technology variables from other clusters. It has the highest score for not wanting to know if \\
& anything is wrong. \\
& Cluster 3 shares similarities with Cluster 2, except for measures related to technology, where it is \\
& more similar to C1. It has the lowest scores for motivation and efficacy type measures than the \\
& other clusters
\end{tabular}

\section{Sub-cluster items and scales put forward to Gravidographic cluster stage}

The items and scales for the variables selected to go forward to the Gravidographic clustering stage were from Pregnancy Health Motivation and Effort and Self Efficacy for Change variables. These variables represent respondent commitment to and perceived ability to make changes. Internal Health Locus of Control was also selected because of its frequent use in health studies and conceptual significance. Wallston et al., (1978) suggests that if a reduction of the full HLOC scale is desired, that the Internal HLOC subscale is used and this was decided at this stage. Affinity for Technology was statistically significant between $\mathrm{C} 2$ and the other clusters; however it was decided to retain this for profiling.

Table 5.76. Other Attitudes and Drivers sub-cluster items/scales put forward to Gravidographic cluster stage

\begin{tabular}{lll}
\hline Construct/attribute & Variable & Item/scale \\
\hline $\begin{array}{l}\text { Other pregnancy attitudes } \\
\text { and drivers }\end{array}$ & Pregnancy health effort and motivation & Pregnancy health effort and \\
& & motivation \\
& Pregnancy Health Locus of control & IHLOC \\
& Enablers and barriers to change & Self efficacy for making health \\
& Approach/avoidance & changes \\
& & Rather not know if something \\
\end{tabular}




\subsubsection{Nutrition construct sub-cluster}

\section{Nutrition sub-cluster scale and item mapping to variables}

The construct Nutrition was sub-clustered on its own as it contained a large amount of variables within it. All nutrition measures were included in the sub-cluster, except the vegetarian sub-variable, as the descriptive results showed that very few women were vegetarians in the sample.

Table 5.77. Nutrition construct sub-cluster scale and item mapping to variables

\begin{tabular}{|c|c|c|}
\hline Variable & Measured with & Scale/item by working title \\
\hline Nutrition Attitudes & $\begin{array}{l}\text { Good nutrition and outcome } \\
\text { beliefs }\end{array}$ & $\begin{array}{l}\text { Keeping advice increases chances of } \\
\text { healthy baby } \\
\text { Eating well reduces chance of small baby } \\
\text { Keeping advice improves changes of self } \\
\text { staying well }\end{array}$ \\
\hline & Eating disturbance & $\begin{array}{l}\text { Developed cravings } \\
\text { Likes and dislikes changed } \\
\text { Morning sickness interferes with eating }\end{array}$ \\
\hline & Nutrition and weight gain & $\begin{array}{l}\text { Well-balanced diet doesn't mind weight } \\
\text { Need to take care re weight gain } \\
\text { Too much weight and health problems } \\
\text { after }\end{array}$ \\
\hline Nutrition practices & $\begin{array}{l}\text { Good Nutrition Effort } \\
\text { Intake against guidelines }\end{array}$ & $\begin{array}{l}\text { Good nutrition effort } \\
\text { Eats } 3 \text { vegetable serves } \\
\text { Eats } 2 \text { fruit serves } \\
\text { Eats } 3 \text { milk serves } \\
\text { Diet low in fat, salt and sugar } \\
\text { Drinks } 6 \text { fluids } \\
\text { Avoids more than } 3 \text { caffeine }\end{array}$ \\
\hline Reducing nutritional risk & $\begin{array}{l}\text { Risky food avoidance } \\
\text { Folic acid profile }\end{array}$ & Avoids risky foods \\
\hline Barriers to good nutrition & Perceived barriers & $\begin{array}{l}\text { Hard to know how to eat healthily } \\
\text { Cannot care as well as would like } \\
\text { No real interest in changing diet } \\
\text { Living situation } \\
\text { Lack of money for food } \\
\text { Lack of skill in food prep } \\
\text { Poor appetite } \\
\text { Poor cooking facilities } \\
\text { Time pressures }\end{array}$ \\
\hline
\end{tabular}


Table 5.78. Nutrition construct sub-cluster distribution

\begin{tabular}{|c|c|c|c|}
\hline Cluster & $\mathbf{N}$ & $\begin{array}{c}\% \text { of } \\
\text { Combine }\end{array}$ & \% of Total \\
\hline 1 & 208 & $44.8 \%$ & $43.5 \%$ \\
\hline 2 & 143 & $30.8 \%$ & $29.9 \%$ \\
\hline 3 & 113 & $24.4 \%$ & $23.6 \%$ \\
\hline Combined & 464 & & $97.1 \%$ \\
\hline Excluded cases & 14 & & $2.9 \%$ \\
\hline Total & 478 & & $100 \%$ \\
\hline
\end{tabular}

Clusters were fixed at three. Cluster 1 is the largest cluster. Cluster 2 and 3 are of similar size.

Nutrition construct sub-cluster Categorical Measures

Table 5.79. Nutrition Sub-Cluster distribution Folic acid intake

\begin{tabular}{lllllll}
\hline Cluster & \multicolumn{2}{l}{ Did not take } & \multicolumn{2}{l}{ Before pregnant } & \multicolumn{2}{l}{ Once pregnancy confirmed } \\
\hline & Frequency & Percent & Frequency & Percent & Frequency & Percent \\
$\mathbf{1}$ & 0 & $0.0 \%$ & 208 & $93.7 \%$ & 0 & $0.0 \%$ \\
$\mathbf{2}$ & 16 & $39.0 \%$ & 0 & $0.0 \%$ & 127 & $63.2 \%$ \\
$\mathbf{3}$ & 25 & $61.0 \%$ & 14 & $6.3 \%$ & 74 & $36.8 \%$ \\
Combined & 41 & $100.0 \%$ & 222 & $100.0 \%$ & 201 & $100.0 \%$ \\
\hline
\end{tabular}

Figure 5.1. Nutrition construct within sub-cluster \% of Folic acid intake

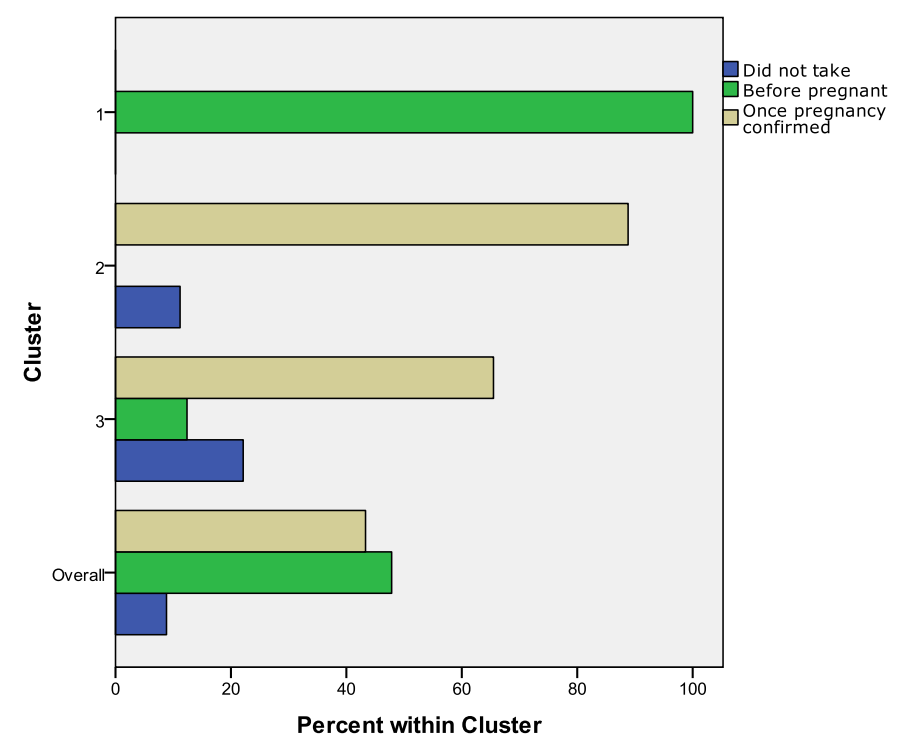

Cluster 1 took Folic acid before pregnancy commenced. Cluster 2 mainly took Folic acid after the pregnancy was confirmed. Cluster 3 is mixed, although most took it once the pregnancy was confirmed. Those who did not take Folic acid are spread between Clusters 2 and 3. 
Table 5.80. Nutrition construct sub-cluster continuous variables

\begin{tabular}{|c|c|c|c|c|c|}
\hline Sub-variable with item/scale below & & Cluster & means & & Information SC Continuous measures: $95 \%$ Confidence Intervals \\
\hline Good nutrition and outcome beliefs & 1 & 2 & 3 & Combined & \\
\hline $\begin{array}{l}\text { Keeping advice increases chances of } \\
\text { healthy baby }\end{array}$ & 5.63 & 5.65 & 4.61 & 5.39 & $\begin{array}{l}\text { C1 and } 2 \text { are above the mean and overlap. C } 3 \text { is below the mean and } \\
\text { significantly different from } \mathrm{C} 1 \text { and } 2\end{array}$ \\
\hline Eating well reduces chance of small baby & 4.76 & 4.63 & 4.13 & 4.57 & $\begin{array}{l}\mathrm{C} 1 \text { and } 2 \text { are above the mean, C3 below. } \mathrm{C} 1 \text { and } 2 \text { overlap. } \mathrm{C} 2 \text { and } 3 \\
\text { overlap. } \mathrm{C} 1 \text { and } 3 \text { are significantly different. }\end{array}$ \\
\hline $\begin{array}{l}\text { Keeping advice improves changes of self } \\
\text { staying well }\end{array}$ & 5.57 & 5.65 & 4.81 & 5.41 & $\begin{array}{l}\mathrm{C} 1 \text { and } 2 \text { are above the mean and overlap. } \mathrm{C} 3 \text { is below the mean and } \\
\text { significantly different from } \mathrm{C} 1 \text { and } 2\end{array}$ \\
\hline \multicolumn{6}{|l|}{ Eating disturbance } \\
\hline Developed cravings & 3.53 & 3.78 & 4.54 & 3.85 & $\begin{array}{l}\mathrm{C} 1 \text { and } 2 \text { are below the mean and } \mathrm{C} 3 \text { above } \mathrm{C} 2 \text { overlaps with the two } \\
\text { other clusters. } \mathrm{C} 1 \text { and } 3 \text { are significantly different. }\end{array}$ \\
\hline Likes and dislikes changed & 3.96 & 3.89 & 4.78 & 4.14 & $\begin{array}{l}\mathrm{C} 1 \text { and } 2 \text { are below the mean and C3 above C2 overlaps with the two } \\
\text { other clusters. } \mathrm{C} 1 \text { and } 3 \text { are significantly different. }\end{array}$ \\
\hline Morning sickness interferes with eating & 4.73 & 4.61 & 4.41 & 4.61 & All clusters overlap. $\mathrm{C} 1$ and two are at the mean, C3 slightly below. \\
\hline \multicolumn{6}{|l|}{ Nutrition and weight gain } \\
\hline Well-balanced diet doesn't mind weight & 4.35 & 4.42 & 4.29 & 4.36 & All clusters are near the overall mean and overlap. \\
\hline Need to take care re weight gain & 4.06 & 3.99 & 3.48 & 3.90 & $\begin{array}{l}\mathrm{C} 1 \text { and } 2 \text { are above the mean, } \mathrm{C} 3 \text { is below the mean. All clusters } \\
\text { overlap. }\end{array}$ \\
\hline Too much weight and health problems after & 4.81 & 4.83 & 4.36 & 4.71 & $\mathrm{C} 1$ and 2 are above the mean, $\mathrm{C} 3$ is below the mean. all overlap. \\
\hline \multicolumn{6}{|l|}{ Good nutrition effort } \\
\hline Good Nutrition Effort & 5.43 & 5.47 & 3.97 & 5.09 & $\begin{array}{l}\mathrm{C} 1 \text { and } 2 \text { are above the mean and overlap. } \mathrm{C} 3 \text { is below the mean and } \\
\text { significantly different from } \mathrm{C} 1 \text { and } 2\end{array}$ \\
\hline \multicolumn{6}{|l|}{ Intake against guidelines } \\
\hline Eats 3 vegetable serves & 5.36 & 5.66 & 3.75 & 5.06 & $\begin{array}{l}\text { C1 and } 2 \text { are above the mean and overlap. } \mathrm{C} 3 \text { is below the mean and } \\
\text { significantly different from } \mathrm{C} 1 \text { and } 2\end{array}$ \\
\hline Eats 2 fruits & 5.95 & 6.06 & 4.73 & 5.69 & $\begin{array}{l}\mathrm{C} 1 \text { and } 2 \text { are above the mean and overlap. } \mathrm{C} 3 \text { is below the mean and } \\
\text { significantly different from } \mathrm{C} 1 \text { and } 2\end{array}$ \\
\hline Eats 3 milk & 5.59 & 5.44 & 4.67 & 5.32 & $\begin{array}{l}\mathrm{C} 1 \text { and } 2 \text { are above the mean and overlap. C3 is below the mean and } \\
\text { significantly different from } \mathrm{C} 1 \text { and } 2\end{array}$ \\
\hline
\end{tabular}




\begin{tabular}{|c|c|c|c|c|c|}
\hline Sub-variable with item/scale below & & Cluster & means & & Information SC Continuous measures: $95 \%$ Confidence Intervals \\
\hline Diet low in fat, salt and sugar & 4.79 & 5.01 & 3.38 & 4.52 & $\begin{array}{l}\mathrm{C} 1 \text { and } 2 \text { are above the mean and overlap. C3 is below the mean and } \\
\text { significantly different from Clusters } 1 \text { and } 2\end{array}$ \\
\hline Drinks 6 fluids & 5.87 & 5.88 & 4.74 & 5.60 & $\begin{array}{l}\mathrm{C} 1 \text { and } 2 \text { are above the mean and overlap. } \mathrm{C} 3 \text { is below the mean and } \\
\text { significantly different from } \mathrm{C} 1 \text { and } 2\end{array}$ \\
\hline Avoids more than 3 caffeine & 6.58 & 6.50 & 5.28 & 6.24 & $\begin{array}{l}\mathrm{C} 1 \text { and } 2 \text { are above the mean and overlap. } \mathrm{C} 3 \text { is below the mean and } \\
\text { significantly different from } \mathrm{C} 1 \text { and } 2\end{array}$ \\
\hline \multicolumn{6}{|l|}{ Risky food avoidance } \\
\hline Avoids risky foods & 6.54 & 6.43 & 5.51 & 6.26 & $\begin{array}{l}\mathrm{C} 1 \text { and } 2 \text { are above the mean and overlap. } \mathrm{C} 3 \text { is below the mean and } \\
\text { significantly different from } \mathrm{C} 1 \text { and } 2\end{array}$ \\
\hline \multicolumn{6}{|l|}{ Perceived barriers } \\
\hline Hard to know how to eat healthily & 2.13 & 2.23 & 3.61 & 2.52 & $\begin{array}{l}\mathrm{C} 1 \text { and } 2 \text { are below the mean and overlap. C3 is above the mean and } \\
\text { significantly different from } \mathrm{C} 1 \text { and } 2\end{array}$ \\
\hline Cannot care as well as would like & 2.56 & 2.52 & 4.00 & 2.90 & $\begin{array}{l}\mathrm{C} 1 \text { and } 2 \text { are below the mean and overlap. } \mathrm{C} 3 \text { is above the mean and } \\
\text { significantly different from } \mathrm{C} 1 \text { and } 2\end{array}$ \\
\hline No real interest in changing diet & 1.51 & 1.48 & 2.84 & 1.83 & $\begin{array}{l}\mathrm{C} 1 \text { and } 2 \text { are below the mean and overlap. } \mathrm{C} 3 \text { is above the mean and } \\
\text { significantly different from } \mathrm{C} 1 \text { and } 2\end{array}$ \\
\hline Living situation & 1.49 & 1.83 & 3.43 & 2.07 & $\begin{array}{l}\mathrm{C} 1 \text { and } 2 \text { are below the mean and overlap. } \mathrm{C} 3 \text { is above the mean and } \\
\text { significantly different from } \mathrm{C} 1 \text { and } 2 \text {. }\end{array}$ \\
\hline Lack of money for food & 1.32 & 1.59 & 3.46 & 1.60 & $\begin{array}{l}\mathrm{C} 1 \text { and } 2 \text { are below the mean and overlap. } \mathrm{C} 3 \text { is above the mean } \\
\text { and significantly different from } \mathrm{C} 1 \text { and } 2\end{array}$ \\
\hline Lack of skill in food prep & 1.25 & 1.37 & 3.32 & 1.79 & $\begin{array}{l}\mathrm{C} 1 \text { and } 2 \text { are below the mean and overlap. } \mathrm{C} 3 \text { is above the mean and } \\
\text { significantly different from Clusters } 1 \text { and } 2\end{array}$ \\
\hline Poor appetite & 2.21 & 2.19 & 3.50 & 2.52 & $\begin{array}{l}\mathrm{C} 1 \text { and } 2 \text { are below the mean and overlap. C3 is above the mean and } \\
\text { significantly different from } \mathrm{C} 1 \text { and } 2\end{array}$ \\
\hline Poor cooking facilities & 1.14 & 1.25 & 2.39 & 1.48 & $\begin{array}{l}\mathrm{C} 1 \text { and } 2 \text { are below the mean and overlap. } \mathrm{C} 3 \text { is above the mean and } \\
\text { significantly different from } \mathrm{C} 1 \text { and } 2\end{array}$ \\
\hline Time pressures & 2.83 & 2.64 & 3.63 & 2.97 & $\begin{array}{l}\mathrm{C} 1 \text { and } 2 \text { are below the mean and overlap. } \mathrm{C} 3 \text { is above the mean and } \\
\text { significantly different from } \mathrm{C} 1 \text { and } 2\end{array}$ \\
\hline
\end{tabular}




\section{Nutrition sub-cluster interpretation}

Clusters 1 and 2 overlap, except for Folic acid intake. Cluster 3 is consistently significantly different from Clusters 1 and 2 .

Table 5.81. Nutrition construct sub-cluster interpretation

\begin{tabular}{ll}
\hline Cluster & Interpretation \\
\hline $\mathbf{1}$ & Cluster 1 is similar to Cluster 2 on almost all measures, except Folic Acid intake. Cluster 1 \\
& respondents took Folic acid before pregnancy, \\
$\mathbf{2}$ & Cluster 2 is similar to Cluster 1 on almost all measures, except Folic Acid intake. Cluster 2 \\
& respondents took Folic acid mostly after pregnancy confirmation \\
$\mathbf{3}$ & Cluster 3 is lowest on nutrition. It is significantly different than 1 and 2. Members report the \\
& highest level of barriers and lower means for nutrition practices. \\
\hline
\end{tabular}

\section{Sub-cluster items and scales put forward to Gravidographic cluster phase}

Good nutrition effort was felt to be reflective of nutrition practices overall and showed similar cluster patterns for nutrition against guidelines items. The two sub-variables within reducing nutrition risk are of conceptual importance as well as demonstrating significant difference between $\mathrm{C} 3$ and the other two clusters. Cluster patterns were the same for both Good nutrition effort and Avoids risky food. It was decided that only Good nutrition effort would go forward to the Gravidographic stage

Table 5.82. Nutrition construct sub-cluster items and scales put forward to Gravidographic cluster phase

\begin{tabular}{lll}
\hline Variable & Sub-variable & Item /scale \\
\hline Nutrition practices & Good nutrition effort & Good nutrition effort \\
Reducing nutritional risk & Folic acid intake & Folic a acid intake \\
\hline
\end{tabular}




\subsubsection{Exercise and Rest construct sub-cluster (Transitional Self sub-domain)}

\section{Scale and item mapping to sub-variables, variables and constructs and attributes}

For the Exercise and Rest sub-cluster, the 'Had an exercise plan' and 'Sources of plan' items were excluded as the majority of women did not have a plan as seen in the descriptive results. When added to the clusters, they strongly influenced the cluster formation, but not in a helpful way. The 'Type of activity engaged in' women undertook variable was excluded, as by far the majority of women had indicated walking as their only activity.

Table 5.83. Exercise and Rest construct sub-cluster scale and item mapping

\begin{tabular}{lll}
\hline Variable & Sub-variable & Scale/item by working title \\
\hline Attitudes about exercise & Benefits & Exercise controls weight \\
& & Regular exercise good for health \\
& & Helps in labour and delivery \\
& & Helps relax and unwind \\
Exercise practices & Information sufficiency & Exercise dangerous for baby \\
Barriers and interference & Patterns & Has right info for exercise \\
& Activity interference & Follows exercise recommendations \\
& Internal barriers and interference & Confused about what to do \\
& & Fctivity interference \\
& & Never exercises anyway \\
& & Too tired or unwell \\
Rest and sleep patterns & Difficult finding time \\
& Rest requirements & Activity uncomfortable \\
& Sleep patterns & Living environment not conducive \\
& External barriers and interference & Work active so no need \\
& & Others discourage \\
& & People put pressure on \\
& & Needs to rest more \\
& & Doesn't sleep as well \\
\hline
\end{tabular}

Table 5.84. Exercise and Rest construct sub-cluster distribution

\begin{tabular}{clll}
\hline Cluster & $\mathbf{N}$ & \% of Combined & \% of Total \\
\hline 1 & 164 & $35.7 \%$ & $34.3 \%$ \\
2 & 142 & $30.9 \%$ & $29.7 \%$ \\
3 & 154 & $33.5 \%$ & $32.2 \%$ \\
Combined & 460 & $100.0 \%$ & $96.2 \%$ \\
Excluded & 18 & & $3.8 \%$ \\
Total & 478 & & $100.0 \%$ \\
\hline
\end{tabular}

The clusters formed spontaneously and were more or less evenly distributed. 
Exercise and Rest construct sub-cluster Categorical Measures

Table 5.85. Exercise and Rest construct sub-cluster: Frequency of exercise

\begin{tabular}{lcccccc}
\hline Cluster & \multicolumn{2}{c}{ Low } & \multicolumn{2}{c}{ Moderate } & \multicolumn{2}{c}{ High } \\
\hline \multirow{3}{*}{ Frequency } & Percent & Frequency & Percent & Frequency & Percent \\
2 & 4 & $4.8 \%$ & 1 & $0.5 \%$ & 159 & $85.9 \%$ \\
3 & 80 & $95.2 \%$ & 36 & $18.8 \%$ & 26 & $14.1 \%$ \\
Combined & 0 & $.0 \%$ & 154 & $80.6 \%$ & 0 & $.0 \%$ \\
& 84 & $100.0 \%$ & 191 & $100.0 \%$ & 185 & $100.0 \%$ \\
\hline
\end{tabular}

Figure 5.2. Exercise and Rest construct within sub-cluster: Frequency of exercise

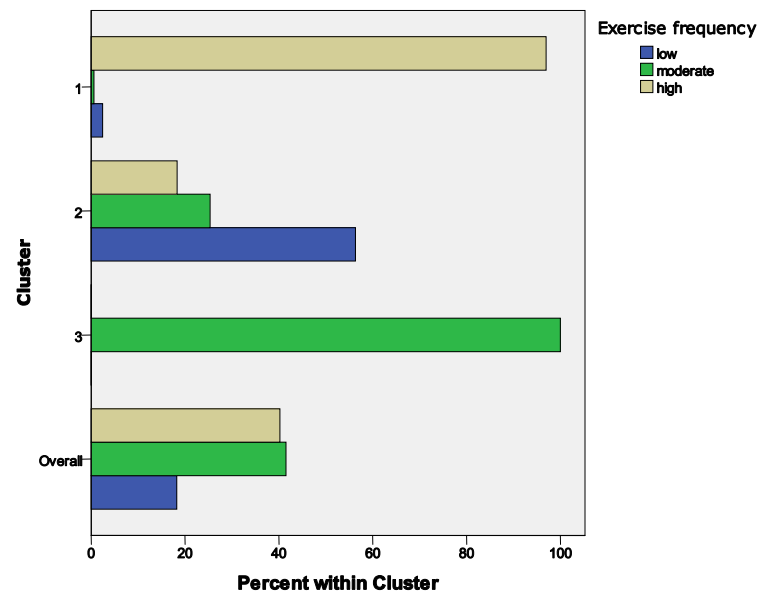

Cluster 1 is made up of mainly high rate exercisers, C2 is mixed, although more low and C3 contains members who exercise moderately. 
Table 5.86. Exercise and Rest construct sub-cluster continuous measures

\begin{tabular}{|c|c|c|c|c|c|}
\hline & \multicolumn{4}{|c|}{ Cluster Means } & \multirow[t]{2}{*}{ Information SC Continuous measures: $95 \%$ Confidence Intervals } \\
\hline Variable with item/scale & $\mathbf{1}$ & 2 & 3 & Combined & \\
\hline \multicolumn{6}{|l|}{ below } \\
\hline \multicolumn{6}{|l|}{ Attitudes toward exercise } \\
\hline Exercise controls weight & 5.59 & 4.51 & 5.18 & 5.12 & $\begin{array}{l}\mathrm{C} 1 \text { and } 3 \text { are above the mean and overlap. } \mathrm{C} 2 \text { is below the mean and } \\
\text { statistically different from } \mathrm{C} 1 \text { and } 3 \text {. }\end{array}$ \\
\hline Exercise is dangerous for baby & 2.10 & 3.01 & 2.14 & 2.40 & $\begin{array}{l}\mathrm{C} 1 \text { and } 3 \text { are below the mean and overlap. } \mathrm{C} 2 \text { is above the mean and } \\
\text { statistically different from } \mathrm{C} 1 \text { and } 3 \text {. }\end{array}$ \\
\hline Helps in labour and delivery & 6.26 & 5.23 & 6.04 & 5.87 & $\begin{array}{l}\mathrm{C} 1 \text { and } 3 \text { are above the mean and overlap. } \mathrm{C} 2 \text { is below the mean and } \\
\text { statistically different from } \mathrm{C} 1 \text { and } 3 \text {. }\end{array}$ \\
\hline Regular exercise good for health & 6.57 & 5.40 & 6.21 & 6.09 & $\begin{array}{l}\mathrm{C} 1 \text { and } 3 \text { are above the mean and overlap. } \mathrm{C} 2 \text { is below the mean and } \\
\text { statistically different from } \mathrm{C} 1 \text { and } 3 \text {. }\end{array}$ \\
\hline Helps relax and unwind & 5.72 & 4.03 & 5.14 & 5.00 & $\begin{array}{l}\mathrm{C} 1 \text { and } 3 \text { are above or on the mean and overlap. } \mathrm{C} 2 \text { is below the mean } \\
\text { and statistically different from } \mathrm{C} 1 \text { and } 3 .\end{array}$ \\
\hline Activity Interference & 3.89 & 3.06 & 3.63 & 3.55 & $\mathrm{C} 1$ and 3 are above the mean. $\mathrm{C} 3$ is below the mean. All clusters overlap. \\
\hline Has right info for exercise & 5.59 & 4.04 & 5.31 & 5.02 & $\begin{array}{l}\mathrm{C} 1 \text { and } 3 \text { are above the mean and overlap. } \mathrm{C} 2 \text { is below the mean and } \\
\text { statistically different from } \mathrm{C} 1 \text { and } 3 \text {. }\end{array}$ \\
\hline \multicolumn{6}{|l|}{ Practices } \\
\hline Follows exercise recommendations & 5.26 & 3.71 & 4.95 & 4.68 & $\begin{array}{l}\mathrm{C} 1 \text { and } 3 \text { are above the mean and overlap. } \mathrm{C} 2 \text { is below the mean and } \\
\text { statistically different from } \mathrm{C} 1 \text { and } 3 \text {. }\end{array}$ \\
\hline \multicolumn{6}{|l|}{ Internal barriers } \\
\hline Confused about what to do & 2.22 & 3.75 & 2.47 & 2.78 & $\begin{array}{l}\mathrm{C} 1 \text { and } 3 \text { are below the mean and overlap. } \mathrm{C} 2 \text { is above the mean and } \\
\text { statistically different from } \mathrm{C} 1 \text { and } 3 \text {. }\end{array}$ \\
\hline Never exercises anyway & 1.41 & 3.08 & 1.90 & 2.09 & $\begin{array}{l}\mathrm{C} 1 \text { and } 3 \text { are below the mean and overlap. } \mathrm{C} 2 \text { is above the mean and } \\
\text { statistically different from } \mathrm{C} 1 \text { and } 3 \text {. }\end{array}$ \\
\hline Difficult finding time & 2.98 & 4.18 & 4.18 & 3.75 & $\begin{array}{l}\mathrm{C} 1 \text { is below the mean and statistically different from } \mathrm{C} 2 \text { and } 3 . \mathrm{C} 2 \text { and } \mathrm{C} 3 \\
\text { are above the mean and overlap. }\end{array}$ \\
\hline Activity uncomfortable & 2.77 & 4.08 & 3.20 & 3.32 & $\begin{array}{l}\mathrm{C} 1 \text { and } 3 \text { are below or on the mean and overlap. } \mathrm{C} 2 \text { is above the mean } \\
\text { and statistically different from } \mathrm{C} 1 \text { and } 3 .\end{array}$ \\
\hline
\end{tabular}




\begin{tabular}{|c|c|c|c|c|c|}
\hline \multirow[b]{2}{*}{ Too tired or unwell } & \multicolumn{3}{|c|}{ Cluster Means } & \multicolumn{2}{|r|}{ Information SC Continuous measures: $95 \%$ Confidence Intervals } \\
\hline & 3.04 & 4.80 & 3.97 & 3.89 & $\begin{array}{l}\text { C1 is below and } 3 \text { on the mean and overlap. C2 is above the mean and } \\
\text { statistically different from } \mathrm{C} 1 \text { and } 3 .\end{array}$ \\
\hline \multicolumn{6}{|l|}{ External barriers } \\
\hline Others discourage & 1.78 & 2.54 & 1.49 & 1.92 & $\begin{array}{l}\mathrm{C} 1 \text { and } 3 \text { are below or on the mean and overlap. } \mathrm{C} 2 \text { is above the mean } \\
\text { and statistically different from } \mathrm{C} 1 \text { and } 3 \text {. }\end{array}$ \\
\hline People put pressure on & 1.82 & 3.05 & 2.40 & 2.39 & $\begin{array}{l}\mathrm{C} 1 \text { and } 3 \text { are below the mean and overlap. } \mathrm{C} 2 \text { is above the mean and } \\
\text { statistically different from } \mathrm{C} 1 \text { and } 3 .\end{array}$ \\
\hline Living environment not conducive & 1.70 & 3.20 & 2.02 & 2.27 & $\begin{array}{l}\mathrm{C} 1 \text { and } 3 \text { are below or on the mean and overlap. } \mathrm{C} 2 \text { is above the mean } \\
\text { and statistically different from } \mathrm{C} 1 \text { and } 3 .\end{array}$ \\
\hline Work active so no need & 2.38 & 3.13 & 2.15 & 2.53 & $\begin{array}{l}\mathrm{C} 1 \text { and } 3 \text { are below the mean and overlap. } \mathrm{C} 2 \text { is above the mean and } \\
\text { statistically different from } \mathrm{C} 1 \text { and } 3 .\end{array}$ \\
\hline \multicolumn{6}{|l|}{ Sleep and rest } \\
\hline Doesn't sleep as well & 5.88 & 5.72 & 5.79 & 5.80 & All clusters overlap. \\
\hline Needs to rest more & 6.13 & 6.15 & 6.31 & 6.20 & All clusters overlap \\
\hline
\end{tabular}




\section{Exercise and Rest construct sub-cluster interpretation}

Cluster 1 and 3 are very similar with difference only in Frequency of exercise. Cluster 2 demonstrates a consistent pattern of falling below Clusters 1 and 2 in regard to exercise attitudes and practices. There is no significant difference found on sleep and rest measures between any of the clusters.

Table 5.87. Exercise and Rest construct sub-cluster interpretation

\begin{tabular}{ll}
\hline Cluster & Interpretation \\
\hline $\mathbf{1}$ & Cluster 1 has almost all the high Frequency of exercise respondents. It is very similar to Cluster 3. \\
& Positive attitudes regarding the benefits of exercise. \\
$\mathbf{2}$ & Cluster 2 has a mixed rate of Frequency of exercise. Respondents have the least positive attitudes \\
& to exercise and perceive significantly higher barriers than Clusters 1 and 3. \\
$\mathbf{3}$ & Cluster 3 is similar to Cluster 1 except it is made up of those respondents whose Frequency of \\
& exercise is moderate.
\end{tabular}

\section{Exercise and Rest sub-cluster items and scales put forward to the Gravidographic cluster phase.}

Variables considered most appropriate for the Gravidographic clustering were first, 'Frequency of exercise', as this was indicative of the actual behaviour engaged in. Secondly, 'Follows exercise recommendations 'demonstrates commitment, whilst activity interference is reflects the extent to which respondents cannot achieve what they would consider a normal level of exercise.

Table 5.88. Nutrition construct sub-cluster items and scales put forward to the Gravidographic cluster phase.

\begin{tabular}{lll}
\hline Variable & Sub-variable & Item/scale \\
\hline Exercise Practices & Patterns & Frequency of exercise \\
& & Follows exercise \\
& & recommendations \\
Barriers and interference to & Activity interference & Activity interference \\
exercise & & \\
\hline
\end{tabular}




\subsubsection{Pregnancy Health Behaviour sub-cluster (constructs derived from Transition Adaptation Adjusters and The Transitional Self sub-domain)}

The Pregnancy Health Behaviour (PHB) sub-cluster is a further progression in reducing the variables in the Physical Health Behaviour sub-domain for the Gravidographic clustering. This sub-cluster chiefly explores how collective pregnancy physical health behaviours cluster. Variables were selected from the Nutrition, Exercise and Rest, Substance Use and Risk and Reducing Risk constructs. In addition, the 'Pregnancy health effort and motivation' variable was also included to explore how this variable clustered amongst overt physical behaviours.

\section{Pregnancy Health Behaviour sub-cluster: Scale and item mapping to variables}

Measures making up this sub-cluster were a collection of variable items and scales that encompassed a holistic view of physical health behaviours present within Physical Transitional Self sub-domain. Therefore, unlike the Exercise and Nutrition sub-clusters that encompassed almost all of the variables within that construct, only a selected few nutrition and exercise variables were taken into the PHB sub-cluster. Folic acid intake was not included in order to see how the clusters formed without it, as it appeared to drive breaking of the clusters in the Nutrition sub-cluster.

Table 5.89. Pregnancy Health Behaviour sub-cluster: Scale and item mapping to variables

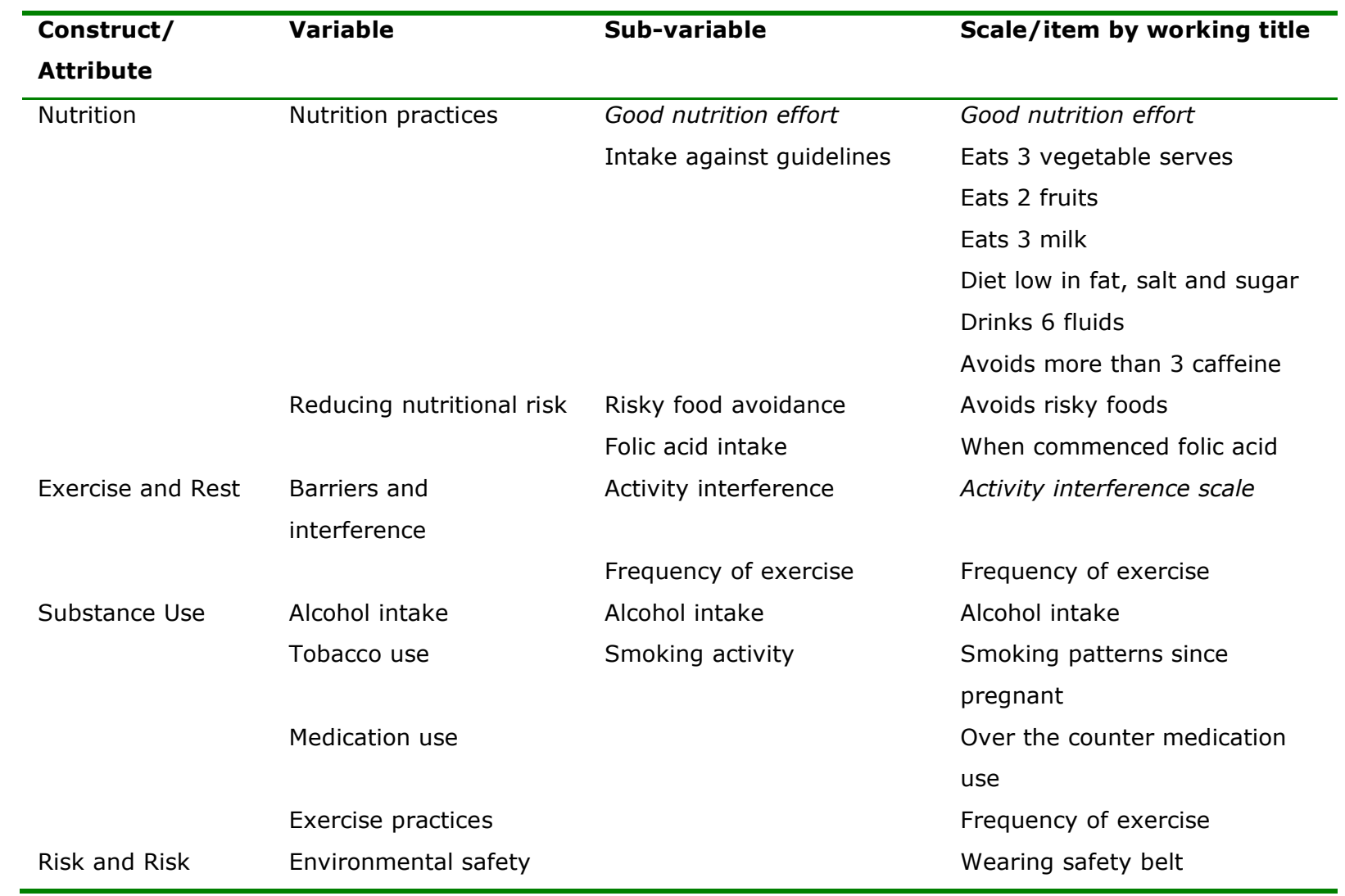




\begin{tabular}{|c|c|c|}
\hline $\begin{array}{l}\text { Construct/ } \\
\text { Attribute }\end{array}$ & Sub-variable & Scale/item by working title \\
\hline \multicolumn{3}{|l|}{ Reduction } \\
\hline & Dental hygiene & Care with dental hygiene \\
\hline $\begin{array}{l}\text { *Major Attitudes } \\
\text { and Drivers }\end{array}$ & $\begin{array}{l}\text { Motivations, } \\
\text { involvement and effort }\end{array}$ & $\begin{array}{l}\text { Pregnancy health effort and } \\
\text { motivation }\end{array}$ \\
\hline
\end{tabular}

*From Transition Adaptation Adjusters

Table 5.90. Pregnancy Health Behaviour sub-cluster: Sub-cluster distribution

\begin{tabular}{clll}
\hline Cluster & $\mathbf{N}$ & $\begin{array}{l}\text { \% of } \\
\text { Combined }\end{array}$ & $\begin{array}{l}\text { \% of } \\
\text { Total }\end{array}$ \\
\hline 1 & 68 & 14.56 & 14.23 \\
2 & 63 & 13.49 & 13.18 \\
3 & 78 & 16.7 & 16.32 \\
4 & 89 & 19.06 & 18.62 \\
5 & 112 & 23.98 & 23.43 \\
6 & 57 & 12.21 & 11.92 \\
Combined & 467 & 100 & 97.7 \\
Excluded Cases & 11 & & 2.30 \\
Total & 478 & & 100 \\
\hline
\end{tabular}

Six spontaneous clusters were derived. Cluster 5 is the largest cluster, whilst C1, C2, C3, C4 and C 6 are a similar size.

Pregnancy Health Behaviour sub-cluster: Categorical measures

Table 5.91. Pregnancy Health Behaviour sub-cluster: Smoking patterns since pregnant

\begin{tabular}{|c|c|c|c|c|c|c|}
\hline \multirow[b]{2}{*}{ Cluster } & \multicolumn{2}{|c|}{ Not smoking before } & \multicolumn{2}{|c|}{$\begin{array}{l}\text { Stopped smoking at } \\
\text { pregnancy }\end{array}$} & \multicolumn{2}{|c|}{ Continues to smoke } \\
\hline & Frequency & Percent & Frequency & Percent & Frequency & Percent \\
\hline 1 & 21 & $6.1 \%$ & 21 & $23.3 \%$ & 26 & $74.3 \%$ \\
\hline 2 & 0 & $0.0 \%$ & 63 & $70.0 \%$ & 0 & $0.0 \%$ \\
\hline 3 & 74 & $21.6 \%$ & 2 & $2.2 \%$ & 2 & $5.7 \%$ \\
\hline 4 & 87 & $25.4 \%$ & 0 & $0.0 \%$ & 2 & $5.7 \%$ \\
\hline 5 & 112 & $32.7 \%$ & 0 & $0.0 \%$ & 0 & $0.0 \%$ \\
\hline 6 & 48 & $14.0 \%$ & 4 & $4.4 \%$ & 5 & $14.3 \%$ \\
\hline Combined & 342 & $100.0 \%$ & 90 & $100.0 \%$ & 35 & $100.0 \%$ \\
\hline
\end{tabular}

Cluster 1 and 2 contains most of those who are smoking at the commencement of pregnancy. Cluster 1 contains almost $75 \%$ of those who continue to smoke. 
Figure 5. 3. Pregnancy Health Behaviour within sub-cluster \%: Smoking patterns since pregnant

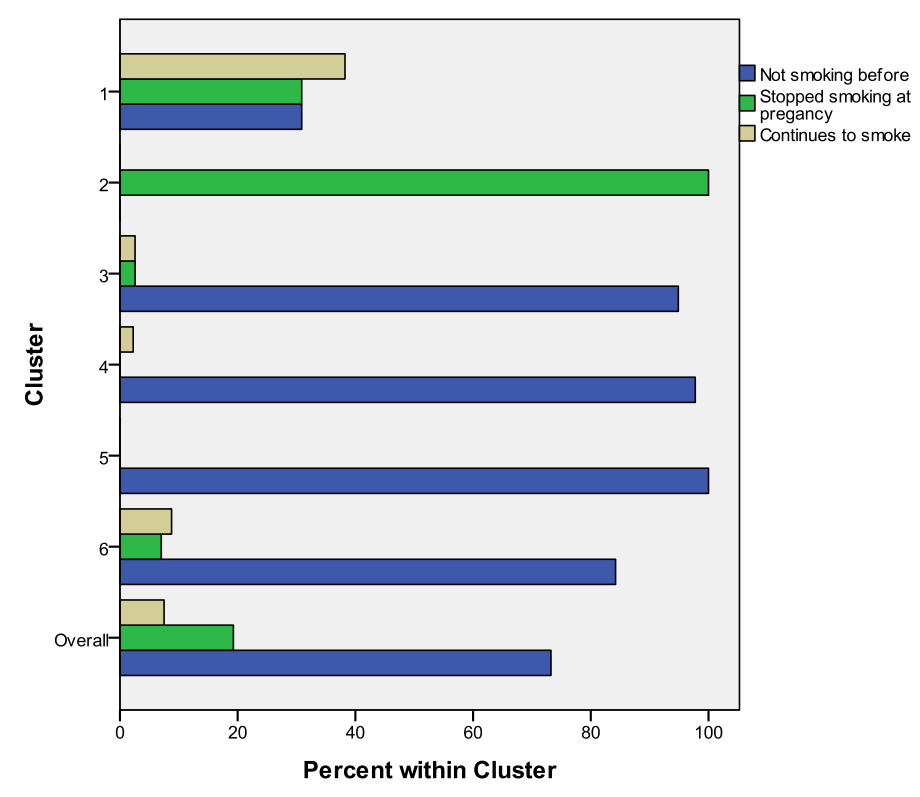

Pregnancy Health Behaviour sub-cluster: Alcohol intake

Table 5.92. Pregnancy Health Behaviour sub-cluster distribution: Alcohol Patterns since pregnant (Drinking or not)

\begin{tabular}{lllll}
\hline & \multicolumn{2}{l}{ Not drinking /stopped } & \multicolumn{2}{l}{ Continues to drink } \\
\hline Cluster & Frequency & Percent & Frequency & Percent \\
1 & 60 & $15.5 \%$ & 8 & $9.9 \%$ \\
2 & 48 & $12.4 \%$ & 15 & $18.5 \%$ \\
3 & 77 & $19.9 \%$ & 1 & $1.2 \%$ \\
4 & 89 & $23.1 \%$ & 0 & $0.0 \%$ \\
5 & 112 & $29.0 \%$ & 0 & $0.0 \%$ \\
6 & 0 & $.0 \%$ & 57 & $70.4 \%$ \\
Combined & 386 & $100.0 \%$ & 81 & $100.0 \%$ \\
\hline
\end{tabular}


Figure 5 4. Pregnancy Health Behaviour within sub-cluster distribution: Alcohol Patterns since pregnant

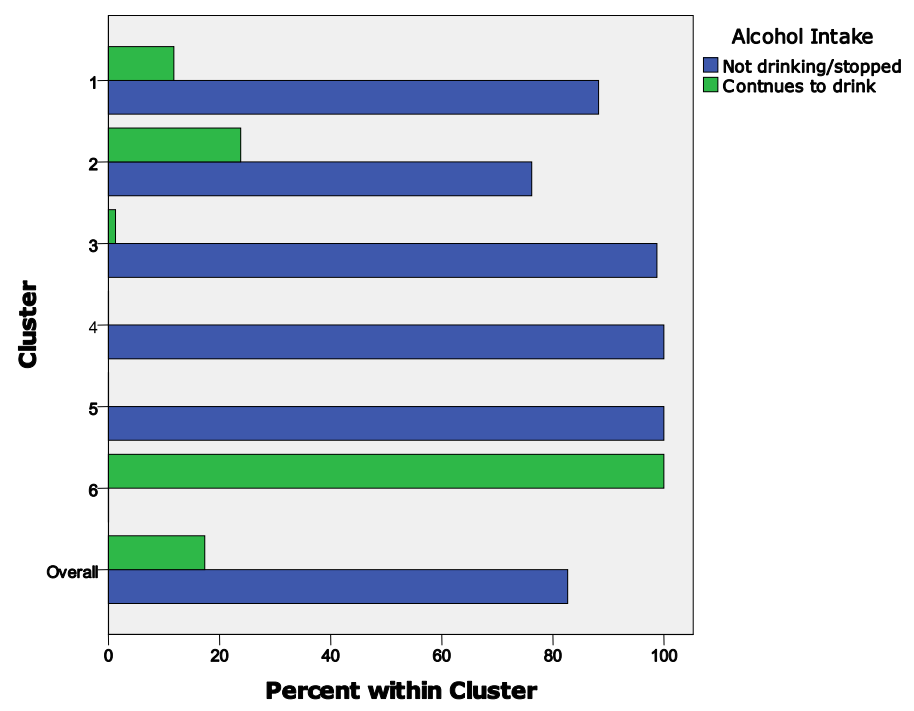

Clusters 2, 3, 4, and 5 have less than $10 \%$ of members who continue to drink alcohol. Cluster 6 makes up $70 \%$ of those who continue to drink.

Table 5.93. Pregnancy Health Behaviour sub-cluster Frequency of exercise

\begin{tabular}{lllllll}
\hline Cluster & \multicolumn{2}{c}{ Low } & \multicolumn{2}{c}{ Moderate } & \multicolumn{2}{c}{ High } \\
\hline \multirow{2}{*}{ Frequency } & Percent & Frequency & Percent & Frequency & Percent \\
1 & 16 & $18.6 \%$ & 30 & $15.3 \%$ & 22 & $11.9 \%$ \\
2 & 14 & $16.3 \%$ & 19 & $9.7 \%$ & 30 & $16.2 \%$ \\
3 & 16 & $18.6 \%$ & 62 & $31.6 \%$ & 0 & $0.0 \%$ \\
4 & 29 & $33.7 \%$ & 60 & $30.6 \%$ & 0 & $0.0 \%$ \\
5 & 0 & $0.0 \%$ & 0 & $0.0 \%$ & 112 & $60.5 \%$ \\
6 & 11 & $12.8 \%$ & 25 & $12.8 \%$ & 21 & $11.4 \%$ \\
Combined & 86 & $100.0 \%$ & 196 & $100.0 \%$ & 185 & $100.0 \%$ \\
\hline
\end{tabular}


Figure 5.5. Pregnancy Health Behaviour within sub-cluster \% Frequency of exercise

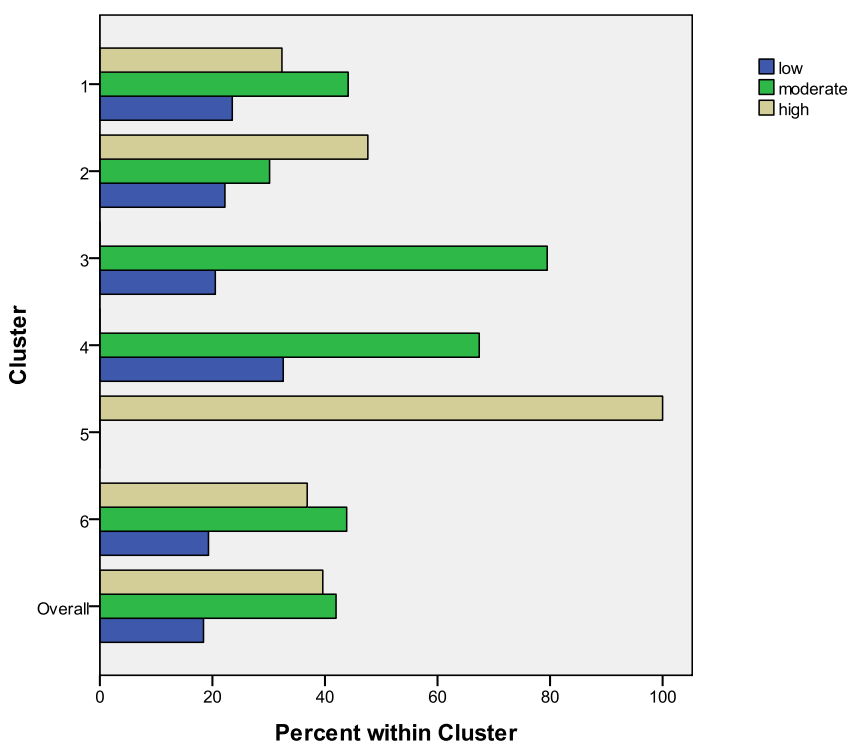

Cluster 5 contains those who have a high frequency of exercise. Clusters 1 and 2 are similar with Frequency of exercise dispersed through the groupings. Clusters 3 and 4 are similar.

Table 5.94. Pregnancy Health Behaviour sub-cluster: Over-the-counter (OTC) medication taken.

\begin{tabular}{lllll}
\hline Cluster & \multicolumn{2}{c}{ No } & \multicolumn{2}{c}{ Yes } \\
\hline & Frequency & Percent & Frequency & Percent \\
1 & 14 & $7.2 \%$ & 54 & $19.9 \%$ \\
2 & 32 & $16.4 \%$ & 31 & $11.4 \%$ \\
3 & 78 & $40.0 \%$ & 0 & $0.0 \%$ \\
4 & 0 & $0.0 \%$ & 89 & $32.7 \%$ \\
5 & 57 & $29.2 \%$ & 55 & $20.2 \%$ \\
6 & 14 & $7.2 \%$ & 43 & $15.8 \%$ \\
Combined & 195 & $100.0 \%$ & 272 & $100.0 \%$ \\
\hline
\end{tabular}


Figure 5.6. Pregnancy Health Behaviour within sub-cluster \% OTC medication taken

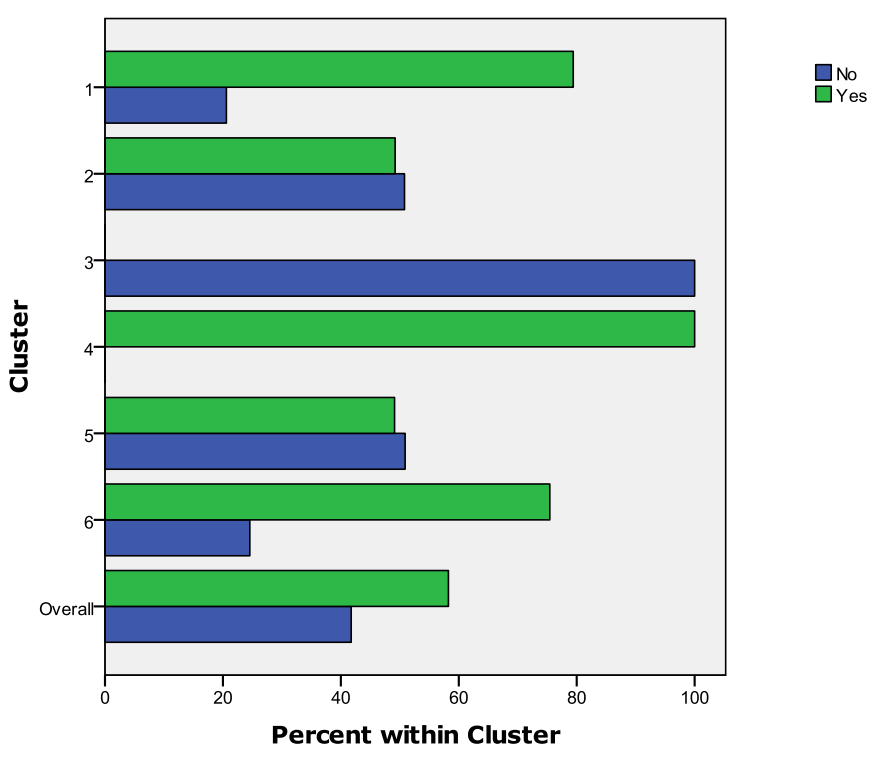

Cluster 4 is made up of those who take OTC medication. Cluster 3 does not take over the counter medication. Cluster 1 and 6 are also more likely to take OTC medication. 
Table 5.95. Pregnancy Health Behaviour sub-cluster: Continuous measures:

\begin{tabular}{|c|c|c|c|c|c|c|c|c|}
\hline \multirow[t]{2}{*}{ Item/scale } & \multicolumn{7}{|c|}{ Cluster means } & \multirow[t]{2}{*}{ Continuous measure: $\mathbf{9 5 \%}$ Confidence Intervals } \\
\hline & 1 & 2 & 3 & 4 & 5 & 6 & Combined & \\
\hline Activity interference & 3.60 & 3.77 & 3.59 & 3.26 & 3.78 & 3.30 & 3.56 & $\begin{array}{l}\text { C4 and } 6 \text { are slightly below the overall mean. However, there is no } \\
\text { significant difference between the clusters. }\end{array}$ \\
\hline Good nutrition effort & 3.81 & 5.32 & 5.37 & 5.06 & 5.45 & 5.21 & 5.07 & $\begin{array}{l}C 1 \text { is significantly different from all other clusters and below the } \\
\text { combined mean. In regard to } C 2,3,4,5 \text { and } 6, C 3 \text { and } 5 \text { have the } \\
\text { highest means. However, there is no significant difference between } C 2 \text {, } \\
3,4,5 \text { and } 6 \text {. }\end{array}$ \\
\hline Eats 3 vege serves & 3.46 & 5.48 & 5.10 & 5.33 & 5.44 & 5.26 & 5.06 & $\begin{array}{l}\text { C1 is below the combined mean and significantly different from all other } \\
\text { clusters. } C 2,3,4,5 \text { and } 6 \text { are not significantly different from each } \\
\text { other. }\end{array}$ \\
\hline Eats 2 fruits & 4.54 & 6.16 & 5.72 & 5.72 & 6.07 & 5.79 & 5.70 & $\begin{array}{l}\mathrm{C} 1 \text { is below the combined mean and significantly different from all other } \\
\text { clusters. } \mathrm{C} 2 \text { and } 5 \text { have the highest means overall. However, } \mathrm{C} 2,3,4,5 \\
\text { and } 6 \text { are not significantly different from each other. }\end{array}$ \\
\hline Eats 3 milk & 4.15 & 5.94 & 5.45 & 5.24 & 5.58 & 5.53 & 5.33 & $\begin{array}{l}\text { C1 is below the mean. All other clusters are above the mean, C1 is } \\
\text { significantly different from all the other clusters, which all overlap }\end{array}$ \\
\hline Drinks 6 fluids & 4.68 & 5.89 & 5.63 & 5.78 & 5.78 & 5.56 & 5.58 & $\begin{array}{l}\mathrm{C} 1 \text { is below the mean. All other clusters are on or above the mean. C2-6 } \\
\text { overlap. }\end{array}$ \\
\hline $\begin{array}{l}\text { Avoids more than } 3 \\
\text { caffeine }\end{array}$ & 4.32 & 6.59 & 6.71 & 6.39 & 6.60 & 6.42 & 6.22 & $\begin{array}{l}\mathrm{C} 1 \text { is below the mean. All other clusters are above the mean, C1 is } \\
\text { significantly different from all the other clusters, which all overlap }\end{array}$ \\
\hline Avoids risky foods & 5.29 & 6.54 & 6.56 & 6.54 & 6.57 & 5.75 & 6.27 & $\begin{array}{l}C 2,3,4 \text {, and } 5 \text { are above the mean and all overlap. } C 1 \text { and } 6 \text { are below } \\
\text { the mean and overlap. }\end{array}$ \\
\hline Wearing safety belt & 6.10 & 6.87 & 6.92 & 6.91 & 6.93 & 6.93 & 6.80 & $\begin{array}{l}\text { C1 is below the mean. All other clusters are above the mean, C1 is } \\
\text { significantly different from all the other clusters, which all overlap. }\end{array}$ \\
\hline Care with dental hygiene & 4.66 & 5.29 & 4.86 & 4.98 & 5.02 & 4.63 & 4.92 & $\begin{array}{l}\mathrm{C} 1 \text { and } 6 \text { are below the mean, C2, 3, } 4 \text { and } 5 \text { are either on or above the } \\
\text { mean. All } 6 \text { clusters overlap }\end{array}$ \\
\hline $\begin{array}{l}\text { Pregnancy health } \\
\text { Effort and motivation }\end{array}$ & 5.68 & 6.38 & 6.17 & 6.12 & 6.13 & 5.86 & 6.07 & $\begin{array}{l}\mathrm{C} 1 \text { and } 6 \text { are below the mean and overlap. } \mathrm{C} 2,3,4 \text { and } 5 \text { are above the } \\
\text { mean and overlap, along with } \mathrm{C} 6 . \mathrm{C} 1 \text { is significantly difference from } \mathrm{C} \\
6 \text {. }\end{array}$ \\
\hline
\end{tabular}




\section{Pregnancy Health Behaviour sub-cluster interpretation}

There is no significant difference between Clusters 2, 3, 4 and 5 in continuous measures. Cluster 1 , is the lowest performing cluster in regards to healthy pregnancy behaviours.

Cluster 6 contains most of those who continue to consume alcohol in pregnancy.

Table 5.96. Pregnancy Health Behaviour sub-cluster interpretation

\begin{tabular}{ll}
\hline Cluster & Interpretation \\
\hline $\mathbf{1}$ & Cluster 1 is lower in most measures than the other clusters and is statistically \\
& different from all the others on many measures. Cluster 1 also contains the highest \\
& number of those who continue to smoke. \\
$\mathbf{2}$ & Clusters 2,34 and 5 are not significantly different on most measures. Cluster 2 \\
& contains the largest number of those who ceased smoking at the commencement of \\
& pregnancy. \\
$\mathbf{3}$ & Clusters 2,34 and 5 are not significantly different on most measures. \\
$\mathbf{5}$ & Clusters 2,34 and 5 are not significantly different on most measures. \\
$\mathbf{6}$ & Cluster 5 respondents all have a high frequency of exercise. \\
& Cluster 6 contains most respondents who drink alcohol. It is more similar to Cluster 1 \\
& than any other clusters
\end{tabular}

\section{Pregnancy Health Behaviour sub-cluster items and scales put forward to Gravidographic cluster stage.}

For nutrition, 'Good nutrition effort' has the same cluster pattern as 'Intake against guidelines' and 'Avoids risky food'. Therefore, 'Good nutrition effort' was selected as to represent nutrition practices. All other variables are held to be salient in the conceptual framework and despite some not being statistically significantly different they are all put forward for the Gravidographic phase. Substance Use again is an importance aspect (especially tobacco and alcohol usage). Over-the-counter medication use appeared relatively distinctive between clusters, and although conceptually perhaps not as salient as smoking and alcohol use, was deemed to be useful for the next clustering phase. Likewise, Risk and Risk Reduction variables and the 'Pregnancy health effort and motivation' variable are extremely salient constructs conceptually. 
Table 5.97. Pregnancy Health Behaviour sub-cluster items and scales put forward to Gravidographic cluster stage.

\begin{tabular}{lll}
\hline Construct/ Attribute & Variable & Item/scale \\
\hline Nutrition & Nutrition practices & Good nutrition effort \\
Substance Use & Alcohol intake & Alcohol intake \\
& Tobacco use & Smoking activity \\
& Medication use & Over the counter medication use \\
Exercise and rest & Attitudes and knowledge & Activity interference \\
& Exercise practices & Follows exercise recommendations \\
& Exercise practices & Frequency of exercise \\
Risk and Risk Reduction & Environmental safety & Wears safety belt \\
& Dental hygiene & Care with dental hygiene \\
Other Attitudes and & Motivations, involvement and & Pregnancy health effort and motivation \\
Drivers & effort & \\
\hline
\end{tabular}

\subsubsection{6 .Psychosocial sub-cluster (The Transitional Self sub-domain)}

\section{Psychosocial sub-cluster scale and item mapping to variables and constructs}

The constructs sub-clustered in the Psycho-social sub-cluster were Coping (Anxiety, Emotional reactions) and Experiencing the Pregnancy Body ('Pregnant body image'). These were considered useful to sub-cluster as they can be considered to be directly related to an individual's own internal resources and attitudes. The constructs Sources support (with variables, Importance from source, Level received from source, Type of support desired, and Living environment) were not included as although they are important influences over health, they are not direct heath behaviours per se. These were kept for profiling of the Gravidographic clusters. 'Appearance interest' was not included as it was felt that a general concept of the pregnant body was sufficiently by captured by 'Pregnant body image'.

Table 5.98. Psychosocial SC scale and item mapping to variables and constructs

\begin{tabular}{lll}
\hline Construct and Attribute & Variable & Items/scale working title \\
\hline Coping & Anxiety & Pregnancy state anxiety \\
& Anxiety & Life situation state anxiety \\
& Emotions & More emotional now \\
Experiencing the Pregnant Body & Pregnant body image & Pregnant body satisfaction \\
& & Weight concerns \\
\hline
\end{tabular}


Table 5.99. Psychosocial SC distribution

\begin{tabular}{llll}
\hline Cluster & $\mathbf{N}$ & \% of Combined & \% of Total \\
\hline 1 & 197 & $41.8 \%$ & $41.2 \%$ \\
2 & 124 & $26.3 \%$ & $25.9 \%$ \\
3 & 150 & $31.8 \%$ & $31.4 \%$ \\
Combined & 471 & $100.0 \%$ & $98.5 \%$ \\
Excluded Cases & 7 & & $1.5 \%$ \\
Total & 478 & & $100.0 \%$ \\
\hline
\end{tabular}

Clusters were fixed at three. Cluster 1 is the largest cluster. Cluster 2 and 3 are similar in size.

\section{Psychosocial SC categorical measures:}

There are no categorical measures in this sub-cluster,

Table 5.100. Psychosocial SC continuous measures

\begin{tabular}{|c|c|c|c|c|c|}
\hline \multirow[t]{2}{*}{ Scale/item } & \multicolumn{4}{|c|}{ Cluster means } & \multirow[t]{2}{*}{ Continuous measures: $95 \%$ Confidence Intervals } \\
\hline & 1 & 2 & 3 & Com & \\
\hline $\begin{array}{l}\text { Pregnancy state } \\
\text { anxiety }\end{array}$ & 3.52 & 5.01 & 4.69 & 4.28 & $\begin{array}{l}\mathrm{C} 2 \text { and } \mathrm{C} 3 \text { are above the overall mean and } \mathrm{C} 1 \text { below. } \\
\text { There is no significant difference between } \mathrm{C} 2 \text { and } 3 . \\
\mathrm{C} 1 \text { is significantly different from the two other clusters. }\end{array}$ \\
\hline $\begin{array}{l}\text { Life situation } \\
\text { state anxiety }\end{array}$ & 2.43 & 4.14 & 4.04 & 3.39 & $\begin{array}{l}\mathrm{C} 2 \text { and } \mathrm{C} 3 \text { are above the overall mean and } \mathrm{C} 1 \text { below. } \\
\text { There is no significant difference between } \mathrm{C} 2 \text { and } 3 \text {. } \\
\mathrm{C} 1 \text { is significantly different from the two other clusters. }\end{array}$ \\
\hline Weight worry & 2.59 & 3.89 & 4.52 & 3.54 & $\begin{array}{l}\mathrm{C} 1 \text { is below the overall mean and } \mathrm{C} 2 \text { and } \mathrm{C} 3 \text { above the } \\
\text { mean. All clusters are significantly different from each } \\
\text { other }\end{array}$ \\
\hline $\begin{array}{l}\text { Pregnancy body } \\
\text { satisfaction }\end{array}$ & 6.22 & 6.13 & 4.30 & 5.58 & $\begin{array}{l}\text { C3 is below the overall mean and Cluster } 1 \text { and } 2 \\
\text { above. } \mathrm{C} 1 \text { and } \mathrm{C} 2 \text { overlap. } \mathrm{C} 3 \text { is significantly different } \\
\text { from } \mathrm{C} 1 \text { and } \mathrm{C} 2 \text {. }\end{array}$ \\
\hline $\begin{array}{l}\text { More emotional } \\
\text { now }\end{array}$ & 4.08 & 6.10 & 4.88 & 4.87 & $\begin{array}{l}\mathrm{C} 1 \text { is below the mean, } \mathrm{C} 2 \text { above and } \mathrm{C} 3 \text { is on the } \\
\text { mean. All clusters are significantly different from each } \\
\text { other. }\end{array}$ \\
\hline
\end{tabular}




\section{Psycho-social SC interpretation}

All the items and scales show patterns that are statistically different between at least one of the clusters and the others.

Table 5.101. Psychosocial SC interpretation

\begin{tabular}{ll}
\hline Cluster & Description \\
\hline C1 & Significantly different from other two clusters on anxiety- experiencing less anxiety/. \\
C2 & C2 is the most emotional, C3 and C2 have similar scores, however all clusters show \\
& significant difference from each tother. \\
C3 & The most concerned regarding weight and with the lowest body image. \\
\hline
\end{tabular}

\section{Psychosocial SC items and scales put forward to Gravidographic stage}

'Pregnancy state anxiety' and 'Life situation state anxiety' share a similar cluster pattern. Pregnancy state anxiety was selected for the Gravidographic clustering as it is more directly related to concerns about pregnancy health. All the other scales and items have been put forward.

Table 5.102. Psychosocial SC items and scales put forward to Gravidographic stage

\begin{tabular}{lll}
\hline Construct/attribute & Variable & Working title \\
\hline Coping & Pregnancy state anxiety & Pregnancy state anxiety \\
& Life situation state anxiety & Life situation state anxiety \\
Experiencing the pregnant body & Pregnant body image & Pregnancy body image \\
& & Weight concerns \\
\hline
\end{tabular}

\subsubsection{Transition Success sub-cluster (SC) (Transition Sucess sub-domain)}

\section{Transition Success SC scale and item mapping to variables and constructs}

As this was a small sub-cluster, data reduction was not applied initially, and all scales and items were sub-clustered.

Table 5.103. Transition Success SC scale and item mapping to variables and constructs

\begin{tabular}{|c|c|c|}
\hline Construct & Variable & Working title \\
\hline Wellbeing & Perception of wellness & Perception of wellness \\
\hline Relationship with baby & Maternal-fetal attachment & Maternal fetal attachment \\
\hline Orientation to the future & Happy to leave childless days behind & $\begin{array}{l}\text { Happy to leave childless days } \\
\text { behind }\end{array}$ \\
\hline
\end{tabular}


Table 5.104. Transition Success SC distribution

\begin{tabular}{llll}
\hline & $\mathbf{N}$ & \% of Combined & \% of Total \\
\hline 1 & 114 & $24.4 \%$ & $23.8 \%$ \\
2 & 187 & $40.0 \%$ & $39.1 \%$ \\
3 & 166 & $35.5 \%$ & $34.7 \%$ \\
Combined & 467 & $100.0 \%$ & $97.7 \%$ \\
Excluded & 11 & & $2.3 \%$ \\
Total & 478 & & $100.0 \%$ \\
\hline
\end{tabular}

Clusters were fixed at three. Cluster 1 is the smallest cluster. Clusters 2 and 3 are similar in size.

\section{Transition Success SC categorical measures}

There are no categorical measures in this sub-cluster.

Table 5.105. Transition Success SC continuous measures

\begin{tabular}{|c|c|c|c|c|c|}
\hline & \multicolumn{4}{|c|}{ Cluster } & \\
\hline & 1 & 2 & 3 & Com & $\begin{array}{l}\text { Transition SC Continuous measures: } \\
95 \% \text { Confidence Intervals }\end{array}$ \\
\hline Perception of wellness & 4.89 & 5.20 & 5.61 & 5.27 & $\begin{array}{l}\text { Cluster } 3 \text { is above the overall mean and } \\
\text { Cluster } 1 \text { and } 2 \text { below. Clusters } 1 \text { and } 2 \\
\text { overlap and are significantly different from } \\
\text { Cluster } 3\end{array}$ \\
\hline $\begin{array}{l}\text { Maternal-fetal } \\
\text { attachment }\end{array}$ & 5.26 & 6.43 & 6.82 & 6.28 & $\begin{array}{l}\text { Clusters } 2 \text { and } 3 \text { are above the overall mean } \\
\text { and Cluster } 1 \text { below. All clusters are } \\
\text { significantly difference from each other }\end{array}$ \\
\hline $\begin{array}{l}\text { Happy to leave } \\
\text { childless days behind }\end{array}$ & 3.58 & 4.53 & 6.48 & 4.99 & $\begin{array}{l}\text { Cluster } 1 \text { and } 2 \text { are below the mean and } \\
\text { Cluster } 3 \text { above. All clusters are significantly } \\
\text { different from each other }\end{array}$ \\
\hline $\begin{array}{l}\text { Looking forward to } \\
\text { new life stage }\end{array}$ & 4.81 & 6.27 & 6.90 & 6.13 & $\begin{array}{l}\text { Cluster } 1 \text { is below the overall mean and } \\
\text { Cluster } 2 \text { and } 3 \text { above. All the clusters are } \\
\text { significantly different from each other. }\end{array}$ \\
\hline
\end{tabular}




\section{Transition Success SC interpretation}

This sub-cluster demonstrates distinctive high, medium and low clusters in regard to significantly different Transition Success measures. However, a degree of caution needs to be taken as although significant difference is demonstrated, in reality some of the mean differences are very small. For instance, means for 'Maternal-fetal attachment' are all above 5 (on a 7 point scale) for all clusters.

Table 5.106. Transition Success SC interpretation

\begin{tabular}{ll}
\hline Cluster & Interpretation \\
\hline $\mathbf{1}$ & Cluster 1 is the smallest. It has the lowest means of all the clusters, apart from perception of \\
& wellness. It is significantly different from all other clusters. It can be said to have the lowest \\
& level of transition success \\
$\mathbf{2}$ & Cluster 2 is the medium of the clusters and maintains this position for all measures. \\
$\mathbf{3}$ & Cluster 3 has the highest means and is significantly different for all measures of Transition \\
& Success. Respondents demonstrate the highest Transition success. \\
\hline
\end{tabular}

\section{Sub-cluster items and scales to Gravidographic cluster stage}

The greatest distinction between the clusters is demonstrated in the anxiety and pregnant body image. These go forward as they are important components of the transition transitional self. 'Looking forward to new life stage' is dropped.

Table 5.107. Transition Success SC items and scales put forward to Gravidographic cluster stage

\begin{tabular}{lll}
\hline Construct/attribute & Variable & Item/scale working title \\
\hline Wellbeing & Perception of wellness & Perception of wellness \\
Relationship with 'baby' & Maternal/fetal attachment & Maternal/fetal attachment \\
Orientation to the future & Leaving childless days behind & Happy to leave childless days behind \\
\hline
\end{tabular}

\section{Final Measures for Gravidographic clustering}

Below is a summary table of the sub-cluster measures extracted from the sub-clustering phase for contribution to the Gravidographic stage. The construct, 'Feelings about the pregnancy' from Pregnancy Circumstance sub-domain is also now included. Items 'Pregnancy intendedness' and 'Evaluation of pregnancy worth' were not involved in the data reduction process of the sub-clustering phase. Although these two constructs have been conceptualised initially as occurring before transition adjustment occurrs, they directly influence and relate to the pregnancy health experience and therefore are salient to the conceptualisation of pregnancy health lifestyle (Speizer et al., 2004; D'Angelo et al., 2004). 
Table 5.108. Summary table of final variables for Gravidographic clustering

\begin{tabular}{|c|c|c|c|c|}
\hline Dimension & Sub-domain & Construct/attribute & Variable & Item/scale working title measure \\
\hline \multirow{3}{*}{$\begin{array}{l}\text { Influencing Lifestyle } \\
\text { Factors }\end{array}$} & Pregnancy Circumstance & Feelings about the Pregnancy & Pregnancy intendedness & Pregnancy intendedness \\
\hline & & & & \\
\hline & & & Evaluation of pregnancy worth & Evaluation of pregnancy worth \\
\hline \multirow[t]{11}{*}{ Life in Transition } & Transition Adaptation & Information Acquisition & Information seeking activity & Information receipt interest \\
\hline & Adjusters & & & \\
\hline & & $\begin{array}{l}\text { Information processing and } \\
\text { utilisation and Utilisation }\end{array}$ & $\begin{array}{l}\text { Perceived information benefits } \\
\text { and outcomes }\end{array}$ & $\begin{array}{l}\text { Perceived positive information } \\
\text { outcomes }\end{array}$ \\
\hline & & $\begin{array}{l}\text { Other Pregnancy Attitudes and } \\
\text { Drivers }\end{array}$ & $\begin{array}{l}\text { Pregnancy health effort and } \\
\text { motivation }\end{array}$ & $\begin{array}{l}\text { Pregnancy health effort and } \\
\text { motivation }\end{array}$ \\
\hline & & & $\begin{array}{l}\text { Pregnancy Health Locus of } \\
\text { Control }\end{array}$ & IHLOC \\
\hline & & & Approach/avoidance & $\begin{array}{l}\text { Rather not know if something is } \\
\text { wrong }\end{array}$ \\
\hline & & & $\begin{array}{l}\text { Enablers and barriers to } \\
\text { change }\end{array}$ & $\begin{array}{l}\text { Self efficacy for making health } \\
\text { change }\end{array}$ \\
\hline & The Transitional self (Physical) & Nutrition & Nutrition practices & Good nutrition effort \\
\hline & & & $\begin{array}{l}\text { Reducing nutritional risk } \\
\text { variable }\end{array}$ & Folic acid intake \\
\hline & & Exercise and rest & Exercise practices & $\begin{array}{l}\text { Exercise patterns } \\
\begin{array}{ll}\text { - } & \text { Frequency of exercise } \\
\text { - } & \text { Follows exercise } \\
& \text { recommendations }\end{array}\end{array}$ \\
\hline & & & $\begin{array}{l}\text { Barriers and interference to } \\
\text { exercise }\end{array}$ & Activity interference \\
\hline
\end{tabular}




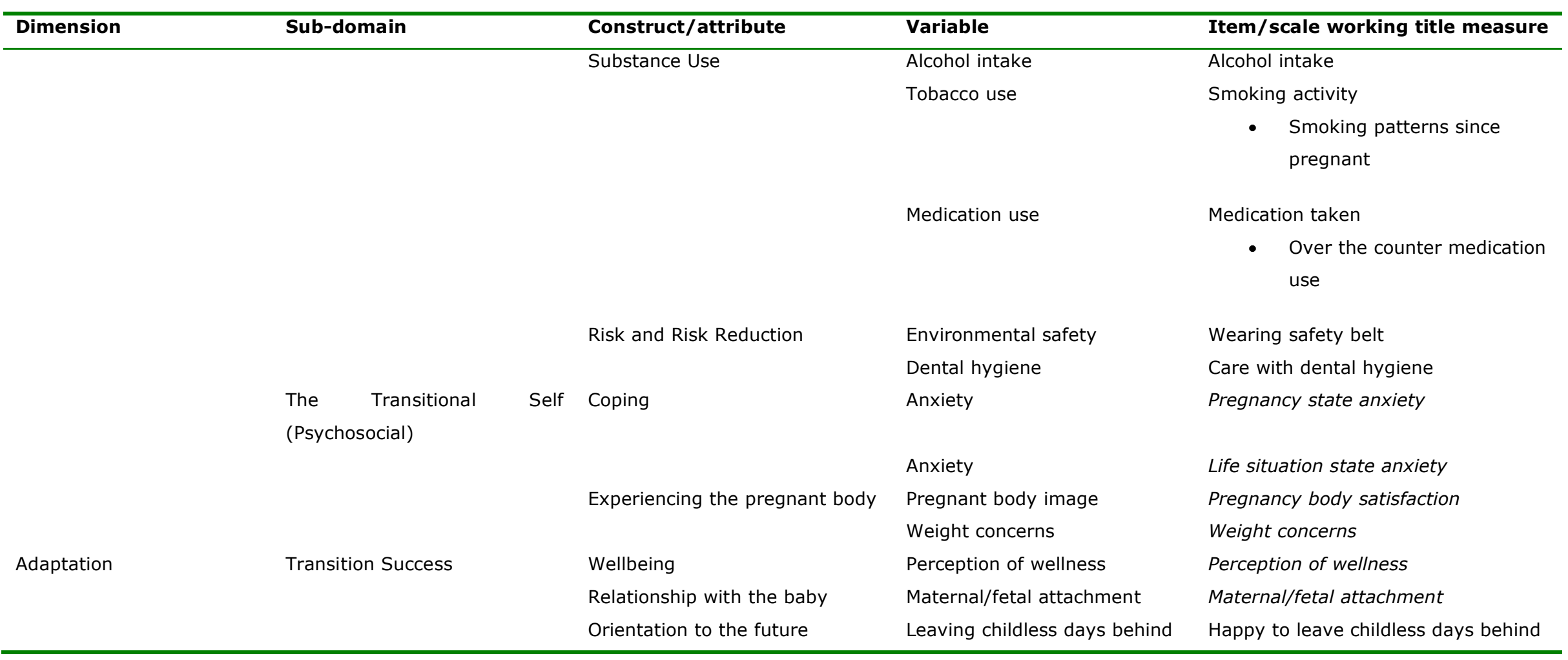




\subsubsection{SUMMARY OF SUB-CLUSTERING PHASE}

The sub-clustering phase was carried out as a way of exploring the data within sub-domains and systems and to gather insights into how health behaviour attitudes and behaviours might cluster. It was also undertaken to reduce the data so that a final clustering phase could be carried out, the Gravidographic stage. This stage was to provide clustering that holistically captured conceptually salient and/or statistically significance variation found during the sub-clustering.

This now ends the section on sub-clustering. The next stage, Gravidographic clustering is presented in Section 5.5.3.

\subsubsection{GRAVIDOGRAPHIC CLUSTERING}

The following sub-section of Chapter 5 presents results of Stages 3, 4 and 5 of the clustering procedure: selection of variables, formation of the Gravidographic clusters and profiling. These pregnancy health lifestyle clusters form the heart of the thesis and segment pregnant women's health lifestyles in a comprehensive yet parsimonious manner. Through data reduction during the sub-clustering phase along with considered judgment, variables that were entered into the Gravidographic cluster stage held a high degree of salience in regard to what comprises the important components of respondent's pregnancy health lifestyles. This enabled identification of segments that had homogeneous characteristics to emerge.

Four Gravidographic lifestyles segments are identified: 'Planners and Pursuers of Health', (Cluster1) 'Mixed Responders', (Cluster 2) 'Lesser Attention to Health' (Cluster3) and 'Slow Starters but Catch Up' (Cluster 4). These clusters will be discussed further at a later stage of Section 5.5.3.

Following the presentation of the statistical results, a description of each cluster is given, followed by profiling of the clusters.

\subsubsection{Gravidographic (GC) Cluster Analysis Results}

The tables following present the cluster distribution for the respondent group, and means and significance for cluster variables. Categorical variables are presented, followed by continuous variables. Four distinct and spontaneous clusters emerged without the need to 'set' or determine the cluster numbers. During the running of clusters, 35 respondents (7.3\%) were excluded. Examination of the data showed no particular pattern for this, other than a random occurrence of 
missing data. Due to the small percentage these made up of the total $(7.3 \%)$, this was considered reasonable. Two clusters ( 1 and 4 ) were almost evenly populated and together held $62.5 \%$ of the sample. Variables used to determine the Gravidographic clusters are presented in order of their placement within the conceptual framework by sub-domains. Categorical variables are presented in tables as percentages. Continuous variables are presented as cluster means. As this stage is the final, definitive stage of the clustering process, some additional information is provided in the sub-cluster stage to aid reader understanding. First, standard deviation scores are given along with cluster means. To aid understanding of how $95 \%$ confidence intervals were determined, error bar charts are given for each continuous variables mean by cluster. The wing bars depict the $95 \%$ confidence limits around each mean.

Table 5.109. Gravidographic (GC) Cluster Membership

\begin{tabular}{ccc}
\hline Cluster & Number & Percentage \\
\hline 1 & 143 & $32.3 \%$ \\
2 & 91 & $20.5 \%$ \\
3 & 75 & $16.9 \%$ \\
4 & 134 & $30.2 \%$ \\
Combined & 443 & $92.6 \%$ \\
Excluded & 35 & $7.3 \%$ \\
Total & 478 & $100 \%$ \\
\hline
\end{tabular}

Cluster 1 and 3 are the largest clusters. Clusters 2 and 3 are smaller and similar in size to each other.

\section{Figure 5.7. Pregnancy Circumstance Gravidographic Cluster variables}

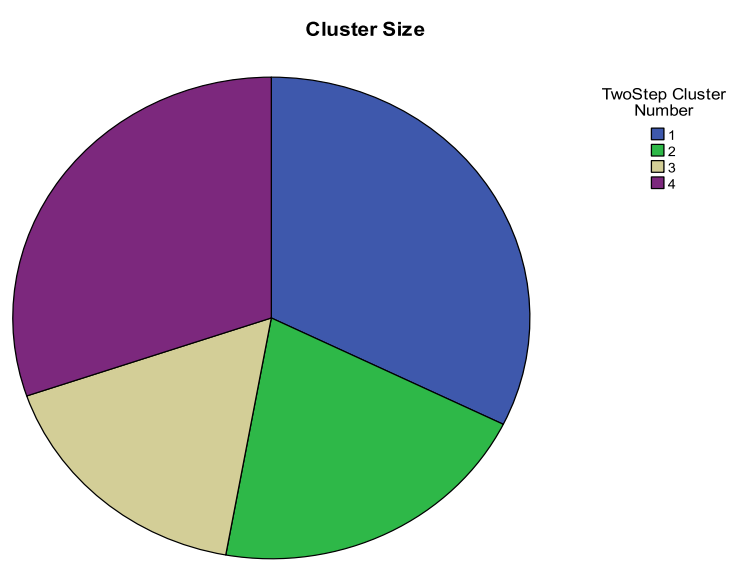


Table 5.110. GC Pregnancy intendedness

\begin{tabular}{|c|c|c|c|c|c|c|c|c|c|c|}
\hline Cluster & Wanted & $\%$ & $\begin{array}{l}\text { Wanted } \\
\text { Earlier }\end{array}$ & $\%$ & $\begin{array}{l}\text { Not } \\
\text { right } \\
\text { away }\end{array}$ & $\%$ & $\begin{array}{l}\text { Not } \\
\text { Want }\end{array}$ & $\%$ & Total & $\%$ \\
\hline 1 & 103 & $72.0 \%$ & 40 & $28.0 \%$ & 0 & $0 \%$ & 0 & $0 \%$ & 143 & 100 \\
\hline 2 & 62 & $68.1 \%$ & 18 & $19.6 \%$ & 9 & $9.9 \%$ & 2 & $2.2 \%$ & 92 & 100 \\
\hline 3 & 6 & $8.0 \%$ & 2 & $2.7 \%$ & 55 & $73.3 \%$ & 12 & $16 \%$ & 75 & 100 \\
\hline 4 & 69 & $51.5 \%$ & 11 & $8.2 \%$ & 53 & $39.6 \%$ & 1 & $0.7 \%$ & 134 & 100 \\
\hline Total & 240 & & 71 & & 117 & & 15 & & 443 & \\
\hline
\end{tabular}

Figure 5.8. GC within cluster \%: Pregnancy intendedness

C1 is made up entirely of 'wanted' or 'wanted to become earlier' pregnancies, that is, those with a pregnancy wish. $\mathrm{C} 2$ is predominately those who 'wanted to become pregnant', or 'earlier' (87.9\%). Those 'wanted, but not right away' were

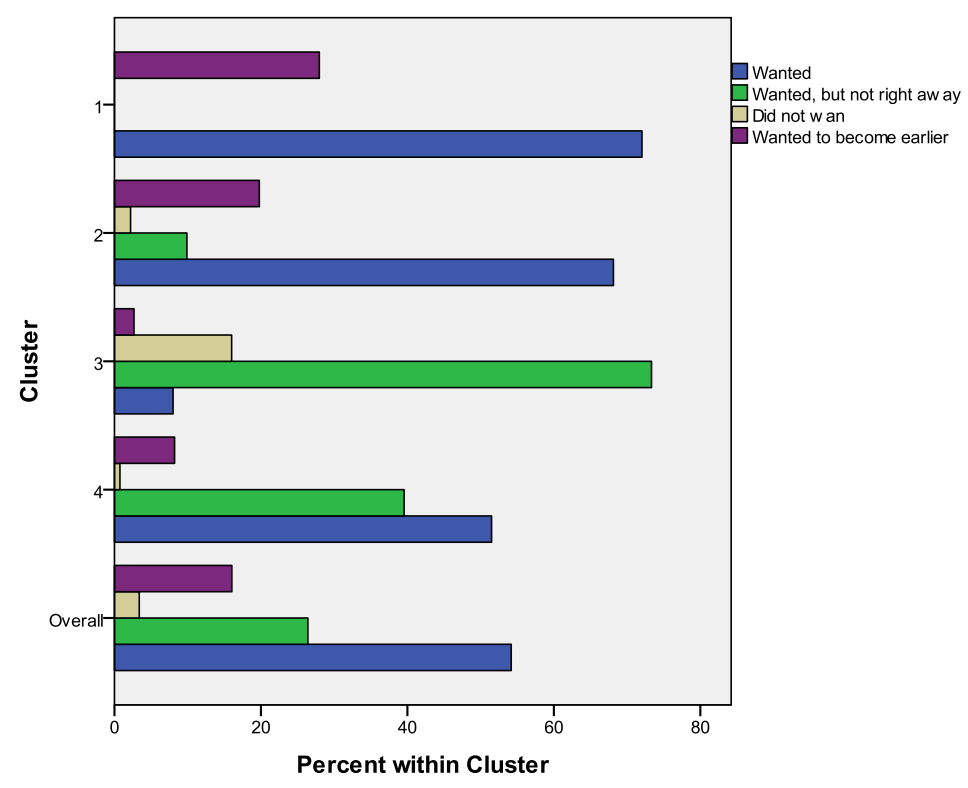
few $(9.9 \%)$ and those who 'did not want' $(2.2 \%)$ very few. C3 is very much made up of 'wanted, but not right away' respondents and captures the largest number of 'did not want' respondents. C4 is made up of a mixture of intention categories. If 'wanted earlier' and 'wanted' are combined however, the group is predominantly those with a pregnancy wish $(59.7 \%)$ versus those without (40.3\%). In summary, C1 and C2 are occupied by planned pregnancies, C3 by 'mistimed' or 'unwanted' and C4 is a mixed group.

Table 5.111. GC Evaluation of pregnancy worth

\begin{tabular}{lllllllll}
\hline Cluster & $\begin{array}{l}\text { Really } \\
\text { wants }\end{array}$ & $\%$ & Is OK & $\%$ & $\begin{array}{l}\text { Not } \\
\text { wanting }\end{array}$ & $\%$ & Total & $\%$ \\
\hline $\mathbf{1}$ & 143 & $100 \%$ & 0 & $0 \%$ & 0 & $0 \%$ & 143 & $100 \%$ \\
$\mathbf{2}$ & 73 & $80.2 \%$ & 18 & $19.8 \%$ & 0 & $0 \%$ & 92 & $100 \%$ \\
$\mathbf{3}$ & 27 & $36 \%$ & 46 & $61.3 \%$ & 2 & $2.7 \%$ & 75 & $100 \%$ \\
$\mathbf{4}$ & 133 & $99.3 \%$ & 1 & $0.75 \%$ & 0 & $0 \%$ & 134 & $100 \%$ \\
\hline Total & 376 & & 65 & & 2 & & 443 & \\
\hline
\end{tabular}


Figure 5.9. GC within cluster \%: Evaluation of pregnancy worth

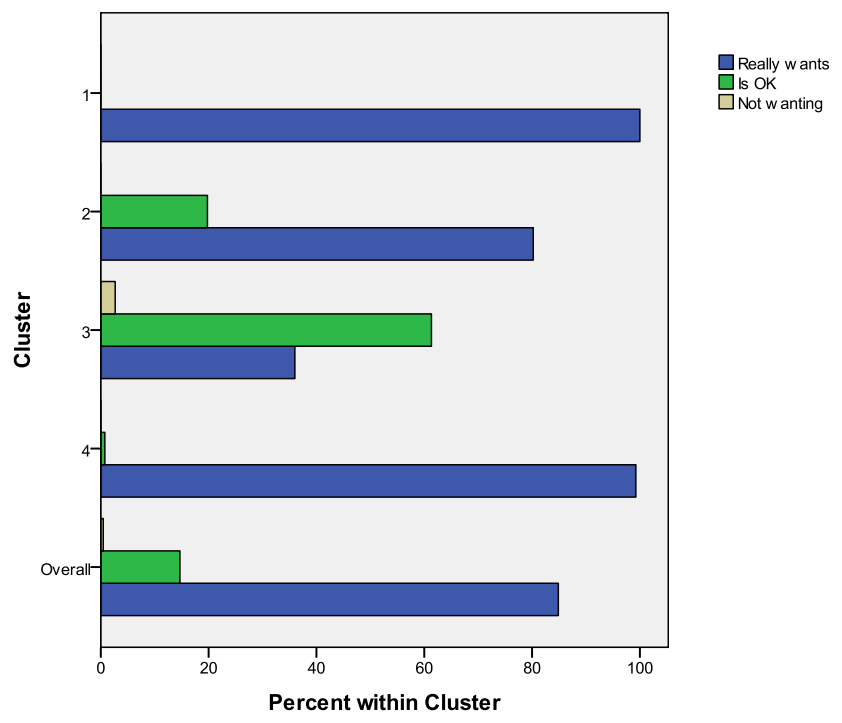

For all respondents (376), $84.9 \%$ now 'really want a baby'. For $\mathrm{C} 1$ and $\mathrm{C} 4$ (minus one member only) all respondents 'really want a baby'. For C2, most are in the 'really want' category, whilst $19.8 \%$ say 'it is $\mathrm{OK}^{\prime}$ For $\mathrm{C} 3$, more respondents say 'it is $\mathrm{ok}^{\prime}$ rather than 'really want a

baby', so this group is mixed. It also contains two members, who say they do not want a baby. Almost all of the 'do not want's from the Intendedness categories have moved into other categories in this table.

\section{Transition Adaptation Adjusters}

Table 5.112. GC Information acquisition and Information processing and utilisation and utilisation

\begin{tabular}{llllllr}
\hline Item/scale & \multicolumn{7}{c}{ Cluster } \\
\hline \multirow{2}{*}{ Info receipt interest } & Mean & $\mathbf{1}$ & $\mathbf{2}$ & $\mathbf{3}$ & $\mathbf{4}$ & Combined \\
& Std. & 0.24 & 6.05 & 5.39 & 6.08 & 6.01 \\
& Dev. & & 0.86 & 1.16 & 0.93 & 0.96 \\
$\begin{array}{l}\text { Perceived positive } \\
\text { information outcomes }\end{array}$ & Mean & 6.02 & 5.49 & 5.37 & 5.96 & 5.78 \\
& Std. & 0.65 & 0.74 & 1.00 & 0.70 & 0.80 \\
& Dev. & & & & & \\
\hline
\end{tabular}

\section{Information receipt interest}

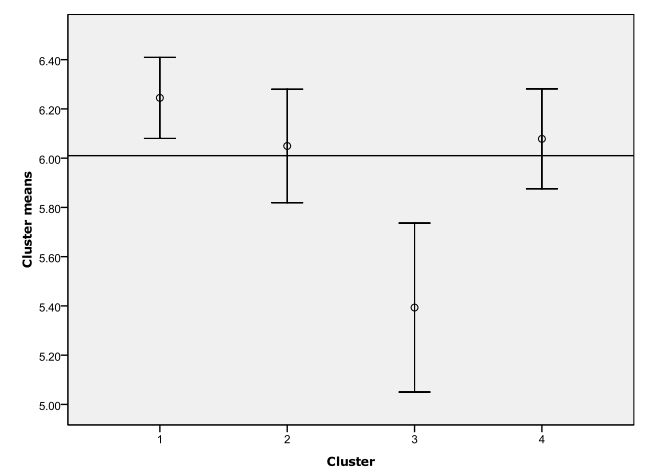

Reference Line is the Overall Mean $=6.01$

For Information receipt interest means are high for all clusters. C1 has the highest mean, C3 the lowest. C1 has the slightly highest mean of all clusters. C1, C2 and C4 overlap. C3 does not overlap with any cluster. 


\section{Perceived positive information outcomes}

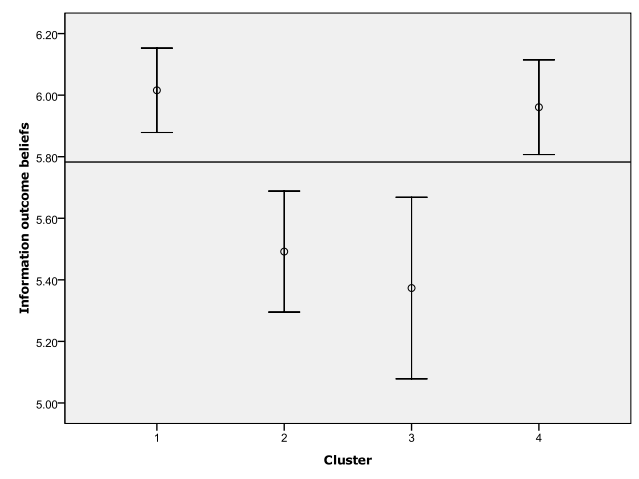

For Perceived positive information outcomes, $\mathrm{C} 1$ and $\mathrm{C} 4$ are equivalent. C2 and $\mathrm{C} 3$ are similar. As for the item above, information interest is high.

Table 5.113. GC Other Pregnancy Attitudes and Drivers

\begin{tabular}{lllccrr}
\hline Item/scale & \multicolumn{5}{c}{ Cluster } \\
\hline \multirow{2}{*}{$\begin{array}{l}\text { Pregnancy health } \\
\text { effort and motivation }\end{array}$} & Mean & $\mathbf{1}$ & $\mathbf{2}$ & $\mathbf{3}$ & $\mathbf{4}$ & Combined \\
& & 6.21 & 5.72 & 5.84 & 6.31 & 6.08 \\
Self efficacy for & Std. Dev. & 0.58 & 0.75 & 0.84 & 0.55 & 0.70 \\
making health changes & Mean & 6.19 & 5.46 & 5.01 & 6.02 & 5.79 \\
& & & & & & \\
Would rather not know & Std. Dev. & 0.71 & 0.96 & 1.08 & 0.81 & 0.97 \\
if something wrong & Std. Dev. & 1.61 & 1.65 & 1.45 & 1.70 & 1.62 \\
Internal HLOC & Mean & 4.68 & 4.20 & 4.83 & 4.85 & 4.66 \\
& Std. Dev. & 0.85 & 0.82 & 1.01 & 0.87 & 0.91 \\
\hline
\end{tabular}

\section{Pregnancy health effort and motivation}

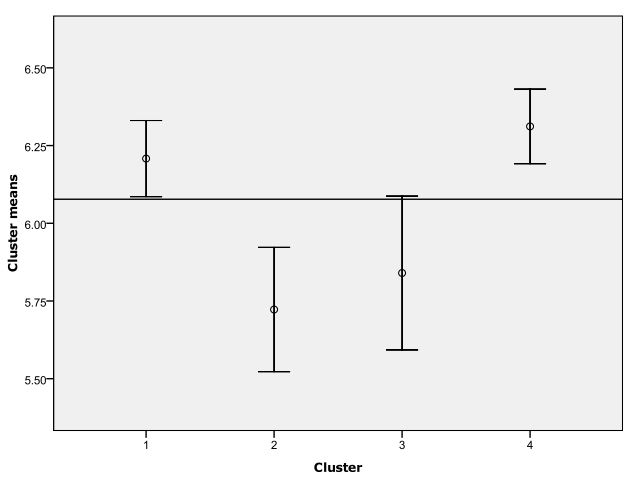

Reference Line is the Overall Mean $=6.08$
For Pregnancy health effort and motivation $\mathrm{C} 1$ and $\mathrm{C} 4$ almost completely overlap and have highest means. C2 and C3 overlap and are below the overall mean. Even though that clusters has a lower mean, the score could still be considered high. 


\section{Self efficacy for making health changes}

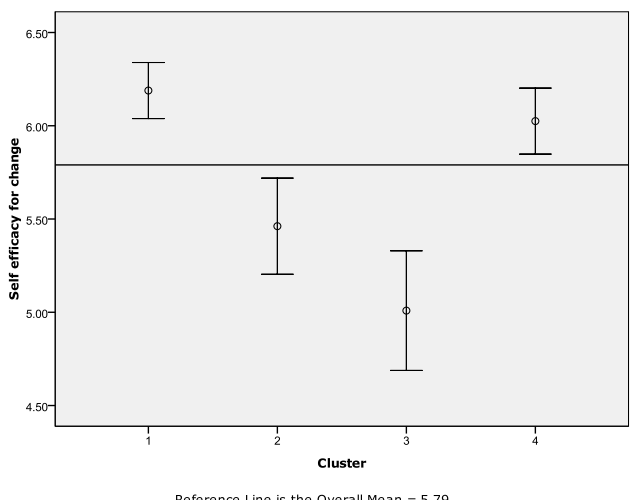

For Self efficacy for making health changes there is a similar pattern to motivation and effort. C1 and C4 overlap, with $\mathrm{C} 1$ having a slightly higher mean. C2 and C3 overlap with means below the overall mean. C1 and C4 do not overlap with C2 and C3. C3 has a 1 point mean below $\mathrm{C} 1$ and $\mathrm{C} 4$. Overall scores in this

section are high for every cluster.

Would rather not know if something wrong

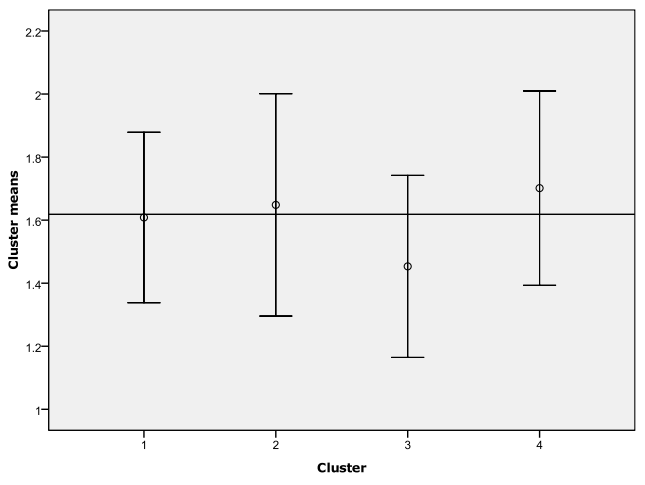

For Would rather not know if something wrong all clusters overlap.

\section{Internal HLOC}

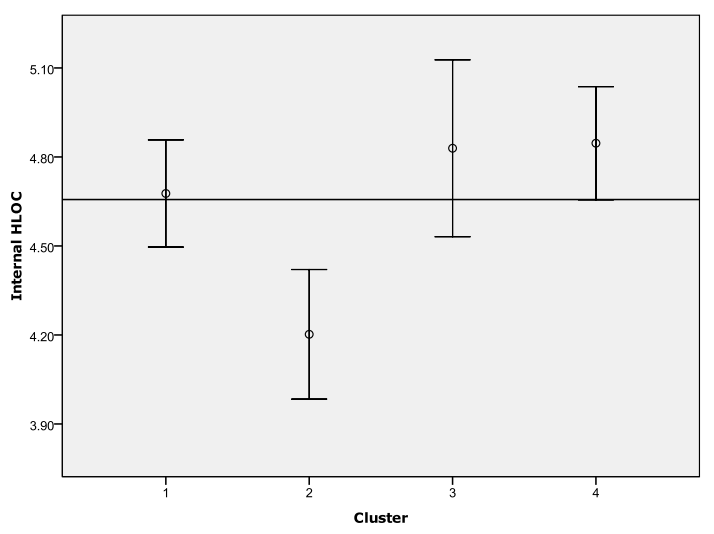

For Internal HLOC C1, C3 and C4 overlap. The C2 mean is below the overall mean and does not overlap with any other cluster. However overall, the actual range for scores is very similar. 
Transitional Self: Physical

\section{Nutrition}

Table 5.114. GC Good nutrition effort

\begin{tabular}{llllllr}
\hline \multicolumn{1}{l}{ Item/scale } & \multicolumn{7}{c}{ Cluster } \\
\hline \multirow{2}{*}{ Good nutrition effort } & & $\mathbf{1}$ & $\mathbf{2}$ & $\mathbf{3}$ & $\mathbf{4}$ & Combined \\
& Mean & 5.58 & 4.92 & 4.05 & 5.26 & 5.09 \\
& Std. Dev. & 0.83 & 0.80 & 1.37 & 0.91 & 1.09 \\
\hline
\end{tabular}

For Good nutrition effort $\mathrm{C} 1$ and $\mathrm{C} 4$ overlap and have mean scores above the overall mean. C2 very slightly overlaps with C4. C3 does not overlap with any other cluster and has a mean below the overall mean, with almost 1 to 1.5 points below the other clusters.

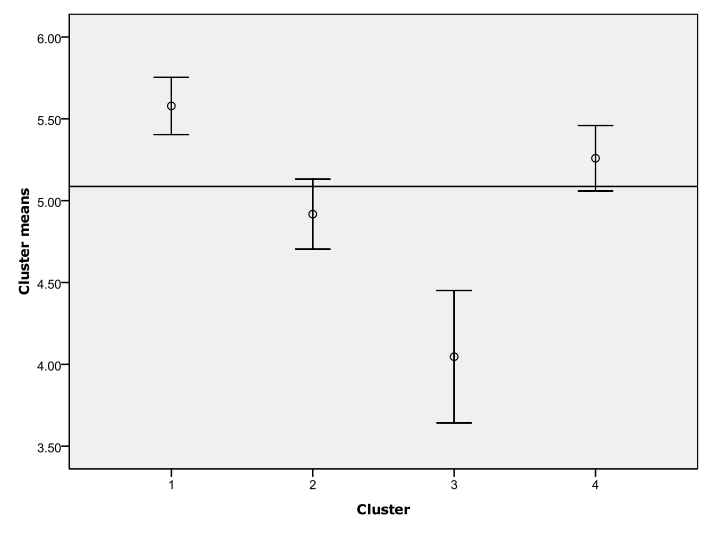

Reference Line is the Overall Mean $=5.09$

Table 5.115. GC Reducing nutrition risk: Folic acid intake

\begin{tabular}{|c|c|c|c|c|c|c|c|c|}
\hline Cluster & Before & $\%$ & $\begin{array}{l}\text { After } \\
\text { confirmed }\end{array}$ & $\%$ & $\begin{array}{l}\text { Did not } \\
\text { take }\end{array}$ & $\%$ & $\begin{array}{l}\text { Total in } \\
\text { cluster }\end{array}$ & $\%$ \\
\hline 1 & 143 & $100 \%$ & 0 & $0 \%$ & 0 & $0 \%$ & 143 & $10 \% 0$ \\
\hline 2 & 63 & $69.2 \%$ & 28 & 30.8 & 0 & $0 \%$ & 91 & $100 \%$ \\
\hline 3 & 1 & $1.3 \%$ & 50 & 66.0 & 24 & 32.0 & 75 & $100 \%$ \\
\hline 4 & 7 & $5.2 \%$ & 113 & 84.3 & 14 & 10.4 & 134 & $100 \%$ \\
\hline Total & 214 & & 191 & & 38 & & 443 & \\
\hline
\end{tabular}

Figure 5.10. GC within cluster \%: Folic acid intake

C1 has $100 \%$ prepregnancy folic acid intake. C2 had a $69.2 \%$ prepregnancy intake and the remainder took folic acid postconfirmation, with no respondents not taking it. C3 had

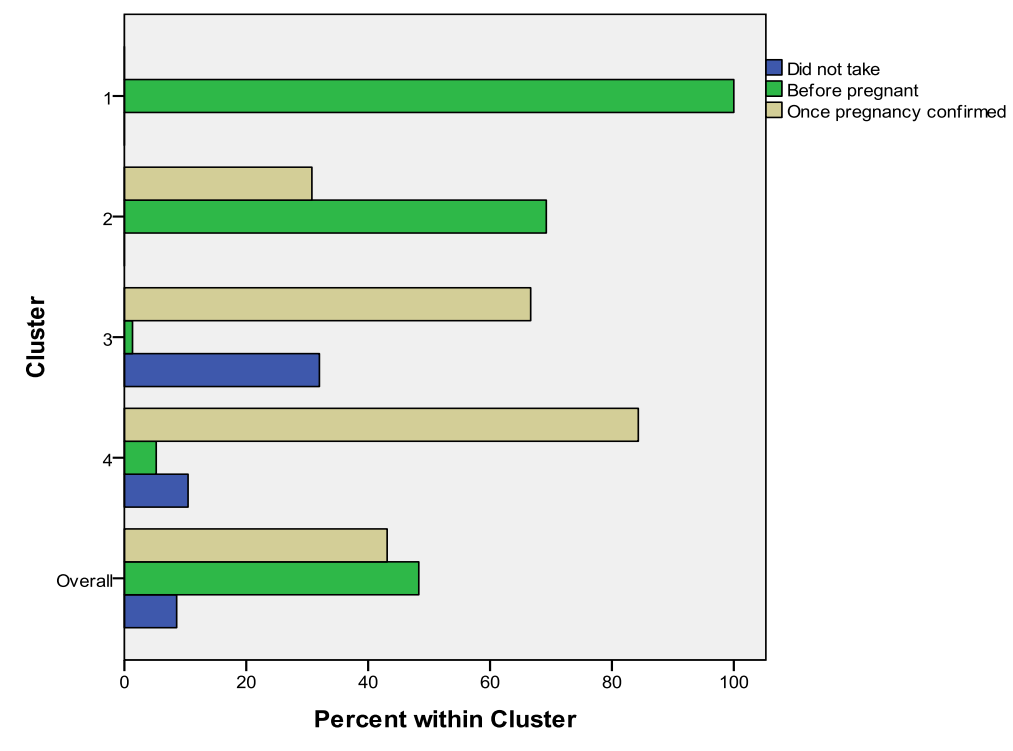


only one respondent taking it pre-pregnancy, meaning that $98 \%$ of C3 respondents had not taken folic acid prior to the onset of pregnancy. C3 has the largest percentage of respondents who 'did not take folic acid'. For C4, few took folic acid pre-pregnancy, with almost all taking it post-pregnancy confirmation. There were some members $(10.4 \%)$, however, who did not take it at all.

\section{Exercise and rest}

Table 5.116. GC Exercise and Rest 'Interference level' and 'Follows exercise recommendations'

\begin{tabular}{llllllr}
\hline Item/scale & \multicolumn{5}{c}{ Cluster } \\
\hline \multirow{2}{*}{ Activity interference } & Mean & $\mathbf{1}$ & $\mathbf{2}$ & $\mathbf{3}$ & $\mathbf{4}$ & Combined \\
& Std. Dev. & 1.58 & 3.10 & 3.74 & 3.73 & 3.55 \\
exercise & Mean & 5.24 & 4.20 & 3.80 & 4.88 & 4.67 \\
recommendations & & & & & & 1.72 \\
& Std. Dev. & 1.19 & 1.57 & 1.55 & 1.25 & 1.46 \\
\hline
\end{tabular}

\section{Activity interference}

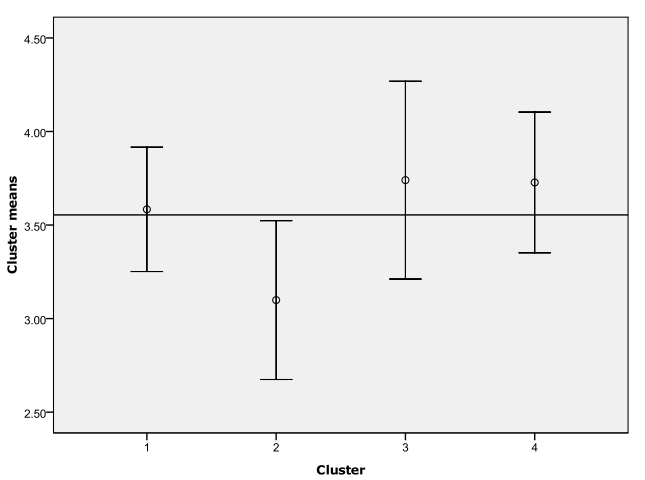

Reference Line is the Overall Mean $=3.55$

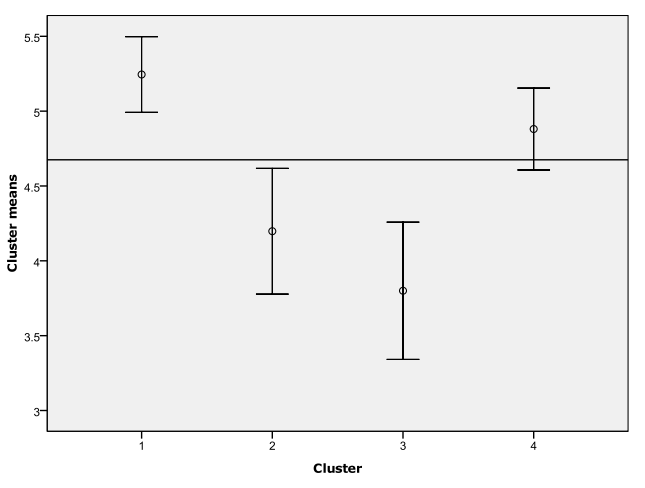

For Activity interference all clusters overlap with similar means. This demonstrates an overall moderate disturbance only to normal activity.

\section{Follows exercise recommendations}

For Follows exercise recomms $\mathrm{C} 1$ and C4 overlap. C2 and C3 overlap with scores below the overall mean. $\mathrm{C} 2$ and $\mathrm{C} 3$ do not overlap with $\mathrm{C} 1$ and $\mathrm{C} 4 . \mathrm{C} 3$ has the lowest mean and is almost 1.5 points below $\mathrm{C} 1$. 
Table 5.117. GC Frequency of exercise

\begin{tabular}{lllllllll}
\hline Cluster & High & \% & Mod & \% & Low & \% & Total & \% \\
\hline $\mathbf{1}$ & 63 & 44.1 & 80 & 56.0 & 0 & 0 & 143 & 100 \\
$\mathbf{2}$ & 31 & 34.1 & 31 & 34.1 & 29 & 31.9 & 92 & 100 \\
$\mathbf{3}$ & 23 & 30.7 & 26 & 34.7 & 26 & 34.7 & 75 & 100 \\
$\mathbf{4}$ & 58 & 43.3 & 50 & 37.3 & 26 & 19.4 & 134 & 100 \\
\hline Total & 175 & & 187 & & 81 & & 443 &
\end{tabular}

Figure 5.11. GC Within cluster \%: Frequency of exercise

C1 is made up of either high or moderate exercisers. C4 has the next highest number with a mix of high and moderate. C4 is occupied by some low exercisers, but they are significantly less than the Moderates and Highs. C2 and C3 are similar with almost equal numbers of the 3

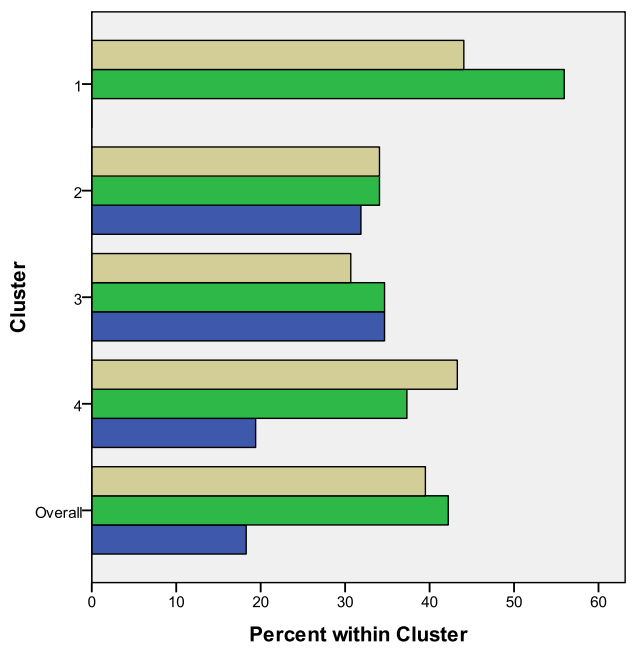
groups.

\section{Substance use}

\section{Tobacco}

Table 5.118. GC Smoking patterns since pregnant

\begin{tabular}{llllllllll}
\hline Cluster & Total & $\begin{array}{l}\text { Not } \\
\text { Smoke }\end{array}$ & \begin{tabular}{l} 
\% \\
\multicolumn{2}{c}{}
\end{tabular} & $\begin{array}{l}\text { Stop } \\
\text { at } \\
\text { preg }\end{array}$ & \% & Continue & \% & Total & \% \\
\hline $\mathbf{1}$ & 143 & 127 & $88.8 \%$ & 14 & $9.8 \%$ & 2 & $1.4 \%$ & 143 & $100 \%$ \\
$\mathbf{2}$ & 91 & 74 & $81.3 \%$ & 14 & $15.4 \%$ & 3 & $3.3 \%$ & 92 & $100 \%$ \\
$\mathbf{3}$ & 75 & 24 & $32,0 \%$ & 32 & $42.6 \%$ & 19 & $25.4 \%$ & 75 & $100 \%$ \\
$\mathbf{4}$ & 134 & 102 & $76.1 \%$ & 23 & $17.2 \%$ & 9 & $6.7 \%$ & 134 & $100 \%$ \\
\hline Total & 443 & 327 & & 83 & & 33 & & 443 & \\
\hline
\end{tabular}


Figure 5.12. GC within cluster \%: Smoking patterns since pregnant

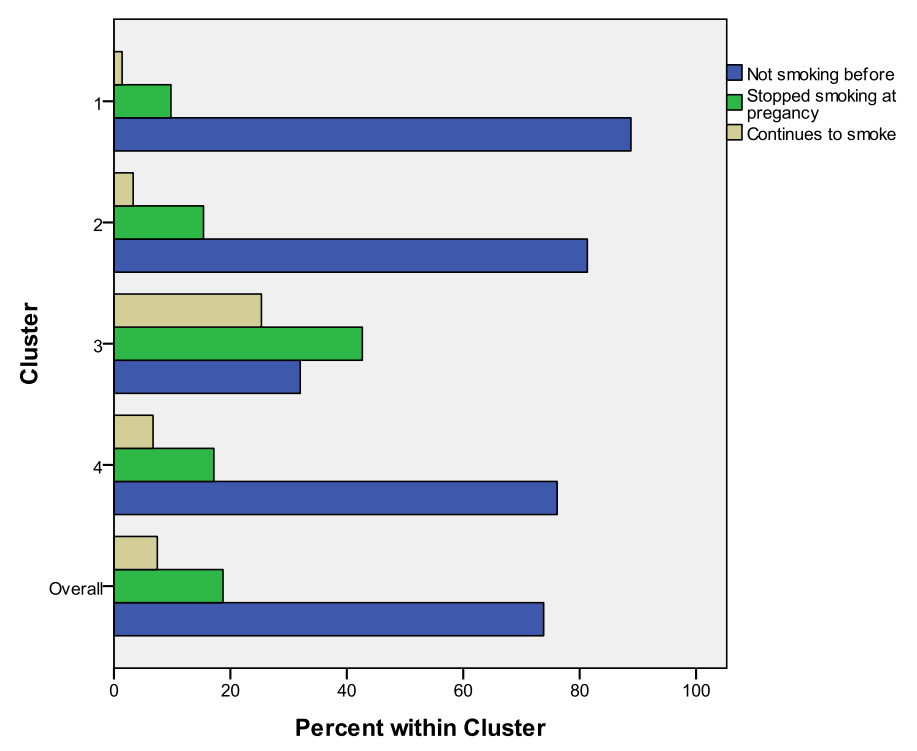

Almost three quarters, $73.9 \%$ of respondents were non-smokers at the onset of pregnancy, $\quad 18.7 \%$ were, but ceased tobacco at the onset of pregnancy, whilst $7.4 \%$ continued to smoke. If 'Stopped at pregnancy' and 'Continues to smoke' are combined, 116 (or $26.2 \%$ ) of all respondents were smokers prior to pregnancy. C1 has the lowest smoking rate. C4 has a significantly high smoking rate (ceasing and continues combined), although does give up overall. C3 has the highest rate of any cluster both for yes bit ceased and yes and continues. C2 and C4 are very similar.

Table 5.119. GC Alcohol intake since pregnant

\begin{tabular}{lllllll}
\hline Cluster & $\begin{array}{l}\text { Not drinking } \\
\text { before/ceased }\end{array}$ & $\%$ & Continues & \% & Total & \% \\
\hline $\mathbf{1}$ & 128 & 89.5 & 15 & 10.5 & 143 & 100 \\
$\mathbf{2}$ & 49 & 53.8 & 42 & 46.2 & 92 & 100 \\
$\mathbf{3}$ & 67 & 89.3 & 8 & 10.6 & 75 & 100 \\
$\mathbf{4}$ & 122 & 91.0 & 12 & 9.0 & 134 & 100 \\
\hline Total & 366 & & 77 & & 443 & \\
\hline
\end{tabular}


Figure 5.13. GC within cluster \%: Alcohol intake since pregnant

Within the cluster results, $82.6 \%$ were not drinking before or ceased to drink at the commencement of the pregnancy. Within Clusters 1,3 and 4 , nearly $90 \%$ or above of respondents cease to drink (or were not drinking before pregnancy). Cluster 2

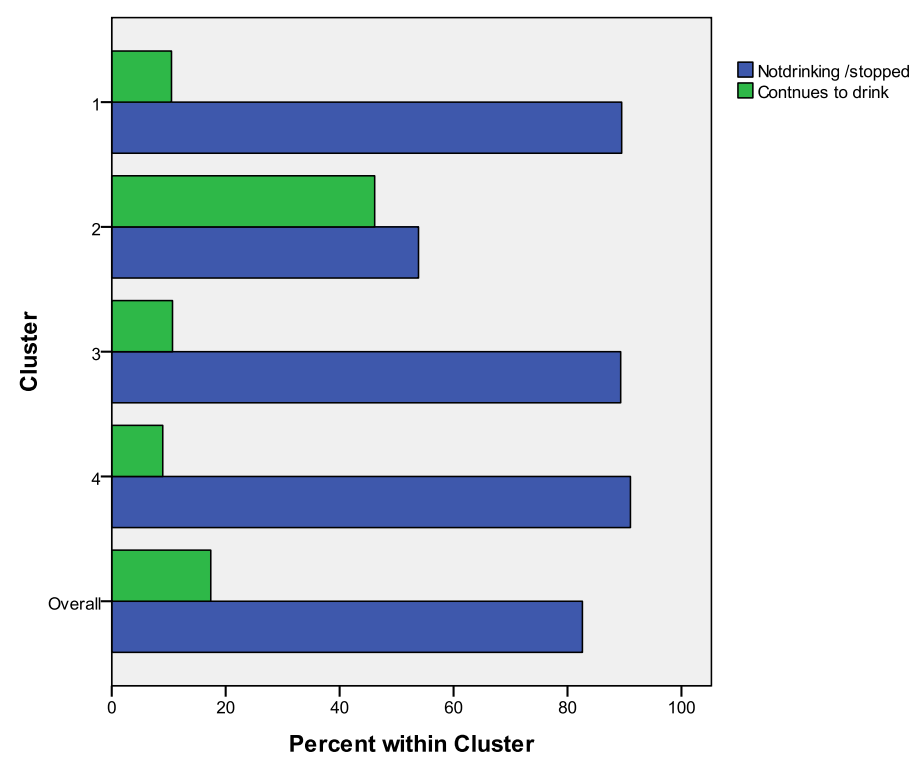
has the highest proportion of drinkers, with $46.2 \%$ continuing and is very different from other clusters

\section{Risk and risk reduction}

Table 5.120 Wearing Safety Belt and Care with Dental Hygiene

\begin{tabular}{llllllr}
\hline Item/scale & \multicolumn{7}{c}{ Cluster } \\
\hline \multirow{2}{*}{ Wearing safety belt } & Mean & $\mathbf{1}$ & $\mathbf{2}$ & $\mathbf{3}$ & $\mathbf{4}$ & Combined \\
& Std. Dev. & 0.92 & 6.90 & 6.24 & 6.92 & 6.80 \\
Care with dental & Mean & 5.17 & 4.27 & 4.67 & 5.28 & 0.76 \\
Hygiene & & & & & & 4.93 \\
& Std. Dev. & 1.38 & 1.67 & 1.69 & 1.32 & 1.53 \\
\hline
\end{tabular}


Wears safety belt

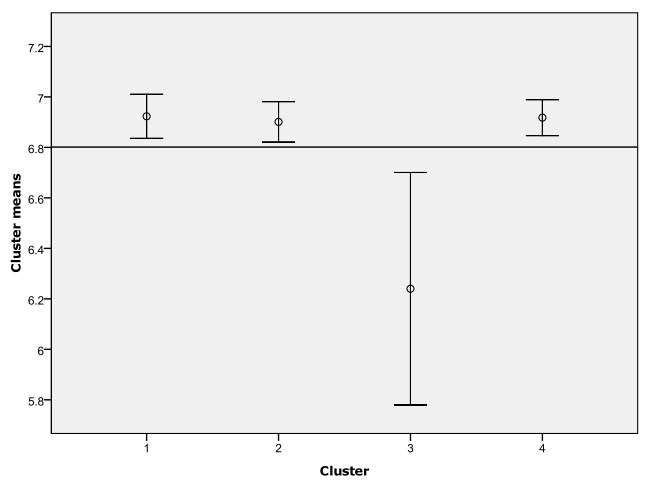

Reference Line is the overall Mean $=7$

\section{Care with dental hygiene}

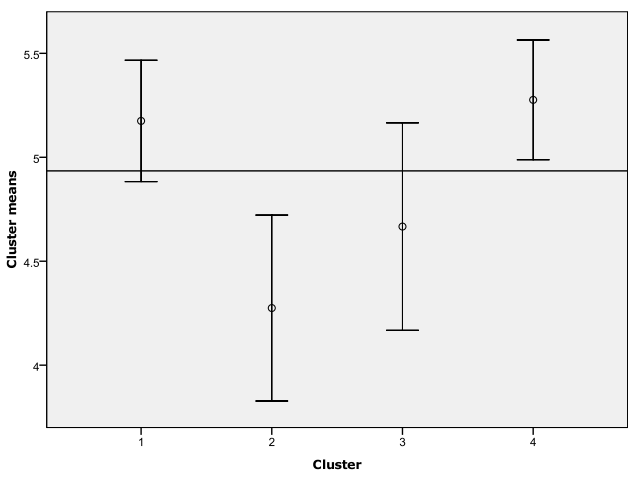

For Wears safety belt, all clusters have a high rate of safety belt wearing. C3 has the lowest mean and has a notable wider deviation. C1, C2 and C4 overlap, C3 does not overlap with any cluster.

For Care with dental hygiene, all clusters overlap with a least 1 other cluster and are overall very close. C2 has the lowest mean and does not overlap with $\mathrm{C} 1$ and $\mathrm{C} 4$. There are quite wide standard deviations.

Transitional self: Psychosocial

Table 5.121: GC Pregnancy State Anxiety, Weight Concern and Pregnancy body satisfaction

\begin{tabular}{llllllr}
\hline Item/scale & \multicolumn{5}{c}{ Cluster } \\
\hline \multirow{3}{*}{ State anxiety } & & $\mathbf{1}$ & $\mathbf{2}$ & $\mathbf{3}$ & $\mathbf{4}$ & Combined \\
& Mean & 3.99 & 4.66 & 4.60 & 4.17 & 4.28 \\
\multirow{2}{*}{ Life situation state anxiety } & Std. Dev. & 1.08 & 1.12 & 1.28 & 1.27 & 1.21 \\
& Mean & 2.90 & 3.70 & 4.18 & 3.33 & 3.41 \\
& Std. Dev. & 1.23 & 1.21 & 1.32 & 1.38 & 1.36 \\
\multirow{3}{*}{ Pregnant worry } & Mean & 3.14 & 4.26 & 4.05 & 3.31 & 3.58 \\
& Std. Dev. & 1.21 & 1.17 & 1.43 & 1.37 & 1.37 \\
& Mean & 5.96 & 4.81 & 4.92 & 5.98 & 5.55 \\
& Std. Dev. & 0.85 & 1.25 & 1.39 & 0.86 & 1.18 \\
\hline
\end{tabular}


For Pregnancy state anxiety moderate for all clusters. $\mathrm{C} 1$ and $\mathrm{C} 4$ are very similar. C1 overlaps with $\mathrm{C} 4$ but no other cluster. C4 overlaps with all 3 other clusters. C2 and C3 have the highest means and are almost equal.

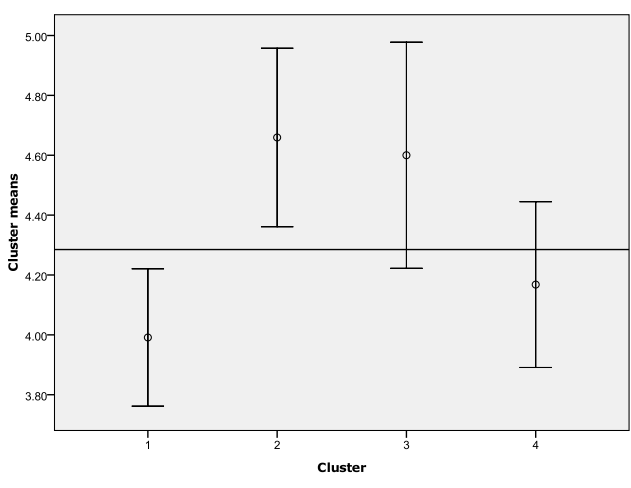

Reference Line is the Overall Mean $=4.28$

\section{Life situation state anxiety}

For Life situation state anxiety similar inter-cluster pattern but means are lower that for Pregnancy State Anxiety. C1 overlaps with C4 but no other cluster. C4 does not overlap with $\mathrm{C} 3$ but does with $\mathrm{C} 1$ and $\mathrm{C} 2$. C3 has the highest mean.

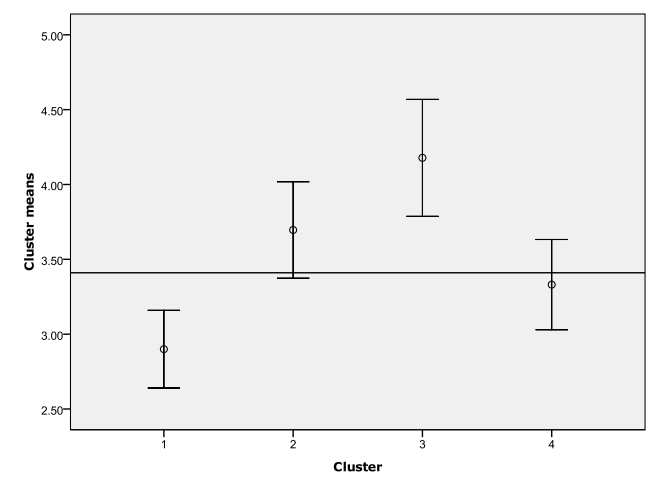

Reference Line is the Overall Mean $=3.41$

\section{Weight worry}

For Weight worry $\mathrm{C} 1$ and C4 are similar. $\mathrm{C} 2$ and $\mathrm{C} 3$ are similar and although only moderate, are above the overall mean and do not overlap with $\mathrm{C} 1$ and $\mathrm{C} 4$.

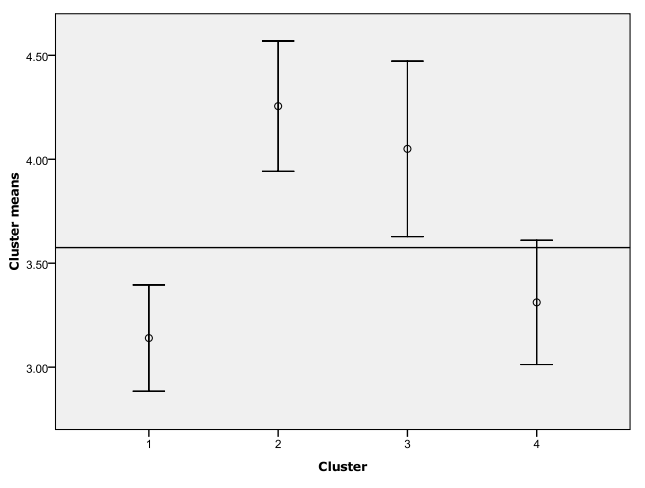

\section{Pregnancy body satisfaction}

For Pregnancy body satisfaction $\mathrm{C} 1$ and C4 are almost equal and slightly above the overall mean. C2 and C3 are similar. C1 and $\mathrm{C} 4$ have the lowest weight

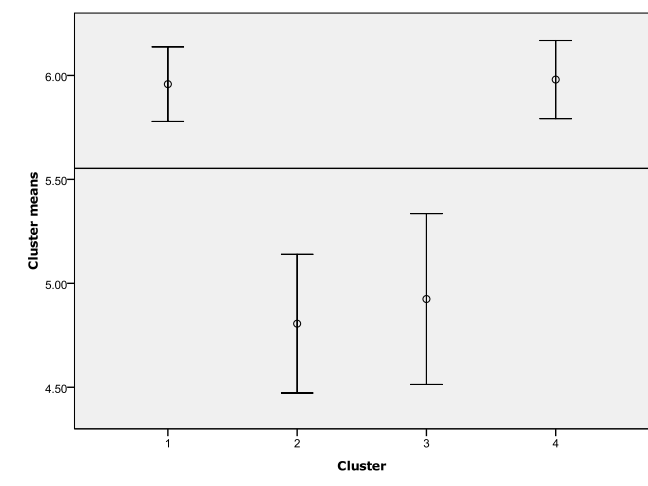


worry and highest body satisfaction combination.

Table 5.122. GC Transition Success

\begin{tabular}{llllllr}
\hline Item/scale & \multicolumn{5}{c}{ Cluster } \\
\hline \multirow{2}{*}{ Maternal/fetal attachment } & & $\mathbf{1}$ & $\mathbf{2}$ & $\mathbf{3}$ & $\mathbf{4}$ & Combined \\
& Mean & 6.51 & 5.73 & 5.79 & 6.65 & 6.27 \\
& Std. Dev. & 0.55 & 0.96 & 1.08 & 0.45 & 0.84 \\
Perception of wellness & Mean & 5.66 & 4.98 & 4.63 & 5.43 & 5.28 \\
& Std. Dev. & 0.70 & 1.03 & 1.03 & 0.76 & 0.93 \\
Happy to leave childless days & Mean & 5.29 & 4.18 & 4.21 & 5.57 & 4.96 \\
behind & & & & & & 1.37 \\
& Std. Dev. & \multirow{2}{*}{1.34} & 1.49 & 1.65 & & 1.55 \\
\hline
\end{tabular}

\section{Maternal/fetal attachment}

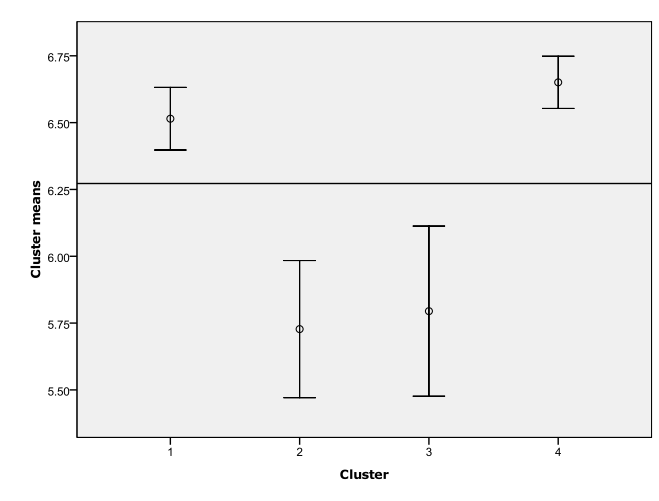

Perception of wellness

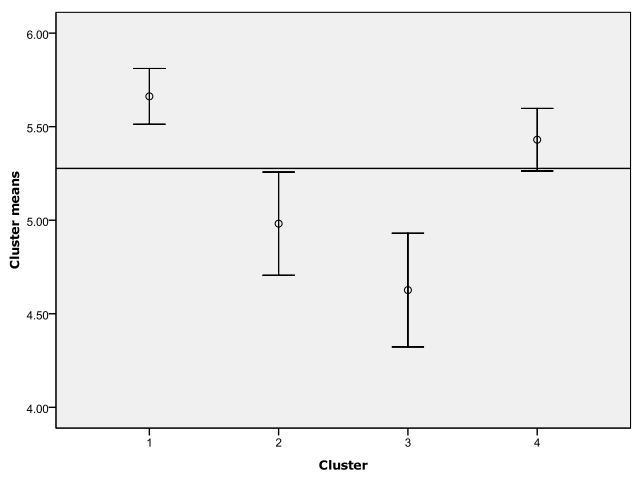

For Maternal/fetal attachment All clusters have high means. C1 and C4 overlap. C2 and C3 are similar and overlap. C1 and C4 are above the overall mean and do not overlap with $\mathrm{C} 2$ and $\mathrm{C} 3$.

For Perception of wellness $\mathrm{C} 1$ and $\mathrm{C} 4$ overlap and are above the overall mean. C2 and C3 overlap and below the overall mean. C3 has lowest mean and C1 the highest.

\section{Happy to leave childless days behind}

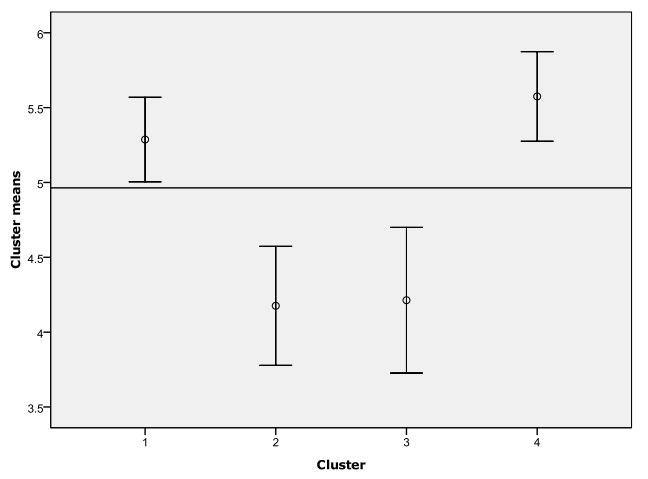

For Happy to leave childless days behind, $\mathrm{C} 1$ and $\mathrm{C} 4$ overlap and are above the mean. C2 and C3 almost completely overlap and are below the mean and both 
are at least 1 point below the means of $\mathrm{C} 1$ and $\mathrm{C} 2$.

\subsubsection{Gravidographic Cluster Descriptions}

The following discussion is based on the findings from the Gravidographic clustering stages for the segments identified and are now described. A title has been given for each segment that is illustrative of the main characteristics that define that group uniquely from other segments.

\section{Cluster 1. 'Planners and strong pursuers of health'}

Cluster 1 ( $\mathrm{C} 1$ ) is populated by respondents who have highly positive pregnancy attitude ratings. They are the best informed and most highly motivated on several measures. The cluster contains $100 \%$ of those with a pregnancy wish and the largest number of respondents in the 'wanted earlier' category. For indicators such as folic acid intake prior to pregnancy, it suggests a degree of planning and modification of health practices prior to pregnancy. If non-smoking is used as one indicator of pre-pregnancy health maintenance, this group is the highest of all groups. C1 can also be said to have an extremely high regard for the pursuit of positive health practices in pregnancy. The 'Maternal-fetal attachment' score is high and body satisfaction high and weight worry low. Transition success measures are also very high.

\section{Cluster 2: 'Mixed responders'}

Cluster 2 (C2) can be said to contain those who know what to do but do not always do it. It is mainly made up of 'wanted' and 'wanted earlier' pregnancies. So for a pregnancy wish, this cluster is quite similar to $\mathrm{C} 1$. However, whilst attitudes are in the higher means, behaviours are toward $\mathrm{C} 3$ at times. It is second to lowest in nutrition and exercise and behaviours tend to tilt toward the lower means. It has by far the highest group of those who continue drinking and differs significantly from other clusters. Although drinking does occur in the other segments to a small degree, this group has 5 times more 'continuing to drink' members than the other segments. However, most members are non-smokers and of those who are smokers at pregnancy onset, almost all cease smoking. C2 has the lowest scores for oral hygiene and shares C3 anxiety, weight worry and transit success measures. Whilst this group has mostly planned pregnancies, some health behaviours are below the overall sample mean. 


\section{Cluster 3: 'Lesser attention to health'}

Cluster 3 (C3) has the least positive behaviours on almost all dimensions and is engaging in the least health promoting behaviour compared to other clusters. Interestingly, compliance to avoidance of alcohol is high. C3 is the cluster that could be said to be most at risk on many dimensions. It has the highest number of 'mistimed' or 'unwanted' pregnancies and the greatest number who continue to smoke. Folic acid intake occurs after pregnancy confirmation, or not at all.

\section{Cluster 4. 'Slow starters but catch up'}

Cluster 4 (C4) is an interesting group. Similar to $\mathrm{C} 1$ in many ways, it is a mix of those with a pregnancy wish and 'mistimed' pregnancies. It has generally positive attitudes and good nutrition and exercise behaviour. The alcohol profile is similar to other clusters (except C2). Few are smoking during the pregnancy. It differs from $\mathrm{C} 1$ in that folic acid intake timing is not as efficient as $\mathrm{C} 1$, with many taking it post-pregnancy confirmation. It is similar to $\mathrm{C} 1$ and $\mathrm{C} 2$ for psychological and transit success. These responders are generally high on a health promoting lifestyle except folic acid timing

\subsubsection{Evaluating the adequacy of the cluster solution}

The data were reduced into four relatively homogenous clusters: two large and two smaller. This represents a high level of parsimony in the final solution relative the amount of data analysed and meets the aims of the study. Meaningful distinctions could be made between each cluster and, despite similarities across some variables, each cluster had distinctive attributes that could be considered unique to the cluster. This is the process advocated and adopted by Berry (2008).

\subsubsection{Checking stability of Gravidographic clusters}

Sub-cluster and Gravidographic cluster stability was checked by reordering the data randomly, therefore starting at a different seed point but specifying the same number of clusters (Hair, 2010). Stable solutions were found, that is with only between ten to twenty percent of cases being assigned to different clusters (Hair, 2010).

\subsubsection{Gravidographic Cluster Profiling}

Cross tabulations and analysis of variance using the selected profiling variables were undertaken to compare the four segments and are now presented in tabular form. In selecting the profiling variables, those that did not appear to have an 
association with health variables (attitudes and behaviours) when tested in the descriptive phase of analysis were excluded from profiling as they did not provide any useful insights.

Profiling variables where a significant difference was observed are discussed first. Following, a table of non-significant variables is presented. This section ends with a summary profiling for each cluster.

\section{Gravidographic clusters (GCs) and Demographics}

\section{Socio-demographic}

Table 5.123. GCs by Age

\begin{tabular}{lllllll}
\hline Age group & \multicolumn{1}{r}{ C1 } & \multicolumn{1}{c}{ C2 } & \multicolumn{1}{c}{ C3 } & \multicolumn{1}{c}{ C4 } & Total \\
\hline $15-24$ & 2 & 6 & 51 & 39 & 98 \\
& $2.0 \%$ & $6.1 \%$ & $52.0 \%$ & $39.8 \%$ & $100.0 \%$ \\
$25-34$ & 110 & 57 & 21 & 78 & 266 \\
& $41.4 \%$ & $21.4 \%$ & $7.9 \%$ & $29.3 \%$ & $100.0 \%$ \\
$34-40+$ & 31 & 28 & 3 & 17 & 79 \\
& $39.2 \%$ & $35.4 \%$ & $3.8 \%$ & $21.5 \%$ & $100.0 \%$ \\
Total & 143 & 91 & 75 & 134 & 443 \\
& $32.3 \%$ & $20.5 \%$ & $16.9 \%$ & $30.2 \%$ & $100.0 \%$ \\
\hline P. $=.000$ & & & & &
\end{tabular}

p. $=.000$

Respondents in the youngest age group are most likely to be in C3, followed by C4. As C3 and C4 are the lowest scores in terms of many health practices, age appears to be influential in choices.

Table 5.124. GCs by Ethnicity

\begin{tabular}{llllll}
\hline Ethnic group & C1 & C2 & C3 & C4 & Total \\
\hline Pakeha/European & 120 & 80 & 40 & 94 & 334 \\
& $35.9 \%$ & $24.0 \%$ & $12.0 \%$ & $28.1 \%$ & $100.0 \%$ \\
Maori/Pacific & 6 & 4 & 25 & 24 & 59 \\
Asian/other & $10.2 \%$ & $6.8 \%$ & $42.4 \%$ & $40.7 \%$ & $100.0 \%$ \\
Total & 17 & 7 & 10 & 15 & 49 \\
& $34.7 \%$ & $14.3 \%$ & $20.4 \%$ & $30.6 \%$ & $100.0 \%$ \\
& 143 & 91 & 75 & 133 & 442 \\
\hline
\end{tabular}

p. $=.000$

C3 has a higher rate of Māori and Pacific respondents than C2 and C1. 
Table 5.125. GCs by Domicile by Size of region

\begin{tabular}{llllll}
\hline Domicile & $\mathbf{C 1}$ & $\mathbf{C 2}$ & $\mathbf{C 3}$ & $\mathbf{C 4}$ & Total \\
\hline Small town & 4 & 6 & 4 & 5 & 19 \\
& $2.8 \%$ & $6.6 \%$ & $5.3 \%$ & $3.8 \%$ & $4.3 \%$ \\
Town & 14 & 10 & 14 & 28 & 66 \\
& $9.8 \%$ & $11.0 \%$ & $18.7 \%$ & $21.1 \%$ & $14.9 \%$ \\
Smaller city & 21 & 15 & 22 & 22 & 80 \\
& $14.7 \%$ & $16.5 \%$ & $29.3 \%$ & $16.5 \%$ & $18.1 \%$ \\
Large city & 104 & 60 & 35 & 78 & 277 \\
& $72.7 \%$ & $65.9 \%$ & $46.7 \%$ & $58.6 \%$ & $62.7 \%$ \\
Total & 143 & 91 & 75 & 133 & 442 \\
& $100.0 \%$ & $100.0 \%$ & $100.0 \%$ & $100.0 \%$ & $100.0 \%$ \\
\hline
\end{tabular}

Fisher's Exact Test .001.

In this sample, C3 and C4 respondents are more likely to live in smaller centres.

Table 5.126. GCs by Educational group

\begin{tabular}{llllll}
\hline Educational group & $\mathbf{C 1}$ & $\mathbf{C 2}$ & $\mathbf{C 3}$ & $\mathbf{C 4}$ & Total \\
\hline College 1-3yrs & 5 & 7 & 23 & 10 & 45 \\
College over 3yrs & $3.5 \%$ & $7.7 \%$ & $31.1 \%$ & $7.5 \%$ & $10.2 \%$ \\
& 16 & 16 & 26 & 32 & 90 \\
Tradecert or Undergrad Dip & 36 & $17.6 \%$ & $35.1 \%$ & $23.9 \%$ & $20.4 \%$ \\
& $25.4 \%$ & $23.3 \%$ & $20.3 \%$ & $23.1 \%$ & $23.8 \%$ \\
Bach or Postgrad Dip & 60 & 36 & 8 & 43 & 147 \\
& $42.3 \%$ & $39.6 \%$ & $10.8 \%$ & $32.1 \%$ & $33.3 \%$ \\
Postgrad degree & 25 & 9 & 2 & 18 & 54 \\
Total & $17.6 \%$ & $9.9 \%$ & $2.7 \%$ & $13.4 \%$ & $12.2 \%$ \\
& 142 & 91 & 74 & 134 & 441 \\
\hline
\end{tabular}

$\mathrm{p}=.000$

C1 and C4 are most likely to hold a post-graduate qualification. C3 is has the highest rate of college education only (this may be a factor of age). C3 tends to have younger respondents, so may not have been able to achieve higher rankings due to age. 
Table 5. 127 GC by Occupational group

\begin{tabular}{llllll}
\hline Occupational group & $\mathbf{C 1}$ & $\mathbf{C 2}$ & $\mathbf{C 3}$ & $\mathbf{C 4}$ & Total \\
\hline Professions & 14 & 8 & 1 & 7 & 30 \\
Commercial prof or manager & 20 & $9.0 \%$ & $1.3 \%$ & $5.3 \%$ & $6.8 \%$ \\
& $14.0 \%$ & 17 & 8 & 17 & 62 \\
Research/science/technician & 22 & 13 & 2 & $12.8 \%$ & $14.1 \%$ \\
& $15.4 \%$ & $14.6 \%$ & $2.7 \%$ & $8.3 \%$ & 11 \\
Teaching/health sciences & 30 & 10 & 2 & 22 & 64 \\
& $21.0 \%$ & $11.2 \%$ & $2.7 \%$ & $16.5 \%$ & $14.5 \%$ \\
Marketing/sales/hospitality & 12 & 6 & 12 & 16 & 46 \\
Clerical & $8.4 \%$ & $6.7 \%$ & $16.0 \%$ & $12.0 \%$ & $10.5 \%$ \\
Factory/trade & 30 & 25 & 13 & 33 & 101 \\
& $21.0 \%$ & $28.1 \%$ & $17.3 \%$ & $24.8 \%$ & $23.0 \%$ \\
At home/beneficiary/student & 7 & 5 & 11 & 16 & 40 \\
& 8 & $5.6 \%$ & $14.7 \%$ & $12.0 \%$ & $9.1 \%$ \\
Total & $4.9 \%$ & $5.6 \%$ & $34.7 \%$ & $8.3 \%$ & $11.1 \%$ \\
& 143 & 89 & 75 & 133 & 440 \\
\hline P & $100.0 \%$ & $100.0 \%$ & $100.0 \%$ & $100.0 \%$ & $100.0 \%$ \\
\hline
\end{tabular}

Professions, technicians and teaching/health occupational groups are most likely to populate $\mathrm{C} 1$. Beneficiaries, students and those at home are most likely to populate C3.

Table 5.128. GCs by Household income

\begin{tabular}{llllll}
\hline $\begin{array}{l}\text { Household } \\
\text { income }\end{array}$ & $\mathbf{C 1}$ & $\mathbf{C 2}$ & $\mathbf{C 3}$ & $\mathbf{C 4}$ & Total \\
\hline$<29,999 \mathrm{k}$ & 5 & 6 & 33 & 26 & 70 \\
& $3.6 \%$ & $6.7 \%$ & $46.5 \%$ & $19.8 \%$ & $16.2 \%$ \\
$30-49,999 \mathrm{k}$ & 17 & 11 & 16 & 27 & 71 \\
& $12.2 \%$ & $12.2 \%$ & $22.5 \%$ & $20.6 \%$ & $16.5 \%$ \\
$50-69,999 \mathrm{k}$ & 21 & 13 & 11 & 27 & 72 \\
& $15.1 \%$ & $14.4 \%$ & $15.5 \%$ & $20.6 \%$ & $16.7 \%$ \\
$70-99,999 \mathrm{k}$ & 31 & 17 & 6 & 23 & 77 \\
$>100,000 \mathrm{k}$ & 65 & $18.9 \%$ & $8.5 \%$ & $17.6 \%$ & $17.9 \%$ \\
& $42.3 \%$ & 43 & 5 & 28 & 141 \\
Total & 139 & 90 & $7.0 \%$ & $21.4 \%$ & $32.7 \%$ \\
& $100.0 \%$ & $100.0 \%$ & $100.0 \%$ & $100.0 \%$ & $100.0 \%$ \\
\hline
\end{tabular}

p.000.

$\mathrm{C} 1$ and $\mathrm{C} 2$ have similar household income, with almost half earning over $\$ 100 \mathrm{~K}$ per year (household). C3 has the least income, almost half earn less that $\$ 50 \mathrm{~K}$ per year. 
Table 5.129. GCs by Employment status

\begin{tabular}{llllll}
\hline Employment status & C1 & C2 & C3 & C4 & Total \\
\hline Full/part time work & 97 & 64 & 30 & 86 & 277 \\
Maternity leave & $68.3 \%$ & $71.1 \%$ & $41.7 \%$ & $64.7 \%$ & $63.4 \%$ \\
Unemployed/seeking work/at home & 27 & 14 & 6 & 19 & 66 \\
& $19.0 \%$ & $15.6 \%$ & $8.3 \%$ & $14.3 \%$ & $15.1 \%$ \\
Student & $5.6 \%$ & $4.4 \%$ & $11.1 \%$ & $11.3 \%$ & $8.0 \%$ \\
& 5 & 3 & 9 & 4 & 21 \\
Sickness beneficiary/other & $3.5 \%$ & $3.3 \%$ & $12.5 \%$ & $3.0 \%$ & $4.8 \%$ \\
Total & 5 & 5 & 19 & 9 & 38 \\
& $3.5 \%$ & $5.6 \%$ & $26.4 \%$ & $6.8 \%$ & $8.7 \%$ \\
\hline
\end{tabular}

Fisher's Exact Test .000

C3 has more sickness beneficiary/other and student members than other clusters. C1, 2 and 4 are similar overall in employment status.

Table 5.130. GCs by Media Use

\begin{tabular}{lll}
\hline Medium & F & P \\
\hline Lifestyle magazines & 13.144 & 0.000 \\
Internet & 10.792 & 0.000 \\
Direct mail & 4.779 & 0.003 \\
Television & 3.836 & 0.01 \\
Playstation / Internet games & 3.836 & 0.000 \\
Women's' magazines & 3.511 & 0.003 \\
DVDs & 3.193 & 0.023 \\
Community papers & 2.923 & 0.034 \\
Business tabloids & 2.525 & 0.057 \\
Newspapers & 1.884 & 0.131 \\
Cinema & 1.132 & 0.336 \\
\hline
\end{tabular}

Table 5.131. GCs by Internet Usage

\begin{tabular}{llllll}
\hline Usage & C1 & C2 & C3 & C4 & Total \\
\hline Low & 11 & 13 & 20 & 14 & 58 \\
& $7.7 \%$ & $14.4 \%$ & $26.7 \%$ & $10.4 \%$ & $13.1 \%$ \\
Mod & 34 & 20 & 33 & 44 & 131 \\
& $23.8 \%$ & $22.2 \%$ & $44.0 \%$ & $32.8 \%$ & $29.6 \%$ \\
High & 98 & 57 & 22 & 76 & 253 \\
& $68.5 \%$ & $63.3 \%$ & $29.3 \%$ & $56.7 \%$ & $57.2 \%$ \\
Total & 143 & 90 & 75 & 134 & 442 \\
& $100.0 \%$ & $100.0 \%$ & $100.0 \%$ & $100.0 \%$ & $100.0 \%$ \\
\hline p.=.000 & & & & &
\end{tabular}


C3 uses the Internet less than other clusters. However, C3's use of Playstation/Internet games is higher that the other clusters. C1 is highest on Lifestyle magazines and lowest on women's magazines amongst clusters.

\section{Gravidographic clusters (GCs) and Social situation}

Table 5.132. GCs by Living with

\begin{tabular}{llllll}
\hline Living with & C1 & C2 & C3 & C4 & Total \\
\hline On my own & 1 & 2 & 4 & 2 & 9 \\
With partner & $0.7 \%$ & $2.2 \%$ & $5.3 \%$ & $1.5 \%$ & $2.0 \%$ \\
With parents & 140 & 85 & 42 & 114 & 381 \\
& $97.9 \%$ & $93.4 \%$ & $56.0 \%$ & $85.1 \%$ & $86.0 \%$ \\
Extended household & 0 & 1 & 21 & 6 & 28 \\
/whanau & $0.0 \%$ & $1.1 \%$ & $28.0 \%$ & $4.5 \%$ & $6.3 \%$ \\
In flat & $0.7 \%$ & $1.1 \%$ & $6.7 \%$ & $5.2 \%$ & $3.2 \%$ \\
Total & 1 & 2 & 3 & 5 & 11 \\
& $0.7 \%$ & $2.2 \%$ & $4.0 \%$ & $3.7 \%$ & $2.5 \%$ \\
& 143 & 91 & 75 & 134 & 443 \\
\hline
\end{tabular}

Fisher's Exact Test .000

C3 members are more likely to be living with parents than other clusters.

Table 5.134. GCs by Contact with Baby's Father

\begin{tabular}{|c|c|c|c|c|c|}
\hline Contact & C1 & $\mathbf{C 2}$ & C3 & C4 & Total \\
\hline \multirow[t]{2}{*}{ No contact } & 0 & 1 & 7 & 7 & 15 \\
\hline & $0.0 \%$ & $1.1 \%$ & $9.3 \%$ & $5.2 \%$ & $3.4 \%$ \\
\hline \multirow{2}{*}{$\begin{array}{l}\text { Not together and } \\
\text { see occasionally }\end{array}$} & 0 & 1 & 4 & 2 & 7 \\
\hline & $0.0 \%$ & $1.1 \%$ & $5.3 \%$ & $1.5 \%$ & $1.6 \%$ \\
\hline \multirow{2}{*}{$\begin{array}{l}\text { Not together but } \\
\text { regular contact }\end{array}$} & 1 & 1 & 13 & 5 & 20 \\
\hline & $0.7 \%$ & $1.1 \%$ & $17.3 \%$ & $3.7 \%$ & $4.5 \%$ \\
\hline \multirow[t]{2}{*}{ Live together } & 142 & 88 & 51 & 120 & 401 \\
\hline & $99.3 \%$ & $96.7 \%$ & $68.0 \%$ & $89.6 \%$ & $90.5 \%$ \\
\hline \multirow[t]{2}{*}{ Total } & 143 & 91 & 75 & 134 & 443 \\
\hline & $100.0 \%$ & $100.0 \%$ & $100.0 \%$ & $100.0 \%$ & $100.0 \%$ \\
\hline
\end{tabular}

Fisher's Exact Test .000

C3 has the most respondents who are not living with the father of the baby. 
Table 5.135. Importance of mother for support

\begin{tabular}{llllll}
\hline Importance level & $\mathbf{C 1}$ & $\mathbf{C 2}$ & $\mathbf{C 3}$ & $\mathbf{C 4}$ & Total \\
\hline Low & 31 & 17 & 10 & 19 & 77 \\
& $23.1 \%$ & $19.1 \%$ & $13.3 \%$ & $15.3 \%$ & $18.2 \%$ \\
Mod & 50 & 45 & 22 & 43 & 160 \\
& $37.3 \%$ & $50.6 \%$ & $29.3 \%$ & $34.7 \%$ & $37.9 \%$ \\
High & 53 & 27 & 43 & 62 & 185 \\
& $39.6 \%$ & $30.3 \%$ & $57.3 \%$ & $50.0 \%$ & $43.8 \%$ \\
Total & 134 & 89 & 75 & 124 & 422 \\
& $100.0 \%$ & $100.0 \%$ & $100.0 \%$ & $100.0 \%$ & $100.0 \%$ \\
\hline
\end{tabular}

$\mathrm{p} .=.001$

C3 and C4 have a higher reliance on their mother for support. This is probably related to a younger age of respondents in these clusters.

Table 5.136. GCs by Happy with living accommodation

\begin{tabular}{llllll}
\hline $\begin{array}{l}\text { Happy with } \\
\text { accommodation }\end{array}$ & C1 & C2 & C3 & C4 & Total \\
\hline No & 7 & 7 & 16 & 15 & 45 \\
& $4.9 \%$ & $7.7 \%$ & $21.6 \%$ & $11.2 \%$ & $10.2 \%$ \\
Yes & 136 & 84 & 58 & 119 & 397 \\
& $95.1 \%$ & $92.3 \%$ & $78.4 \%$ & $88.8 \%$ & $89.8 \%$ \\
Total & 143 & 91 & 74 & 134 & 442 \\
& $100.0 \%$ & $100.0 \%$ & $100.0 \%$ & $100.0 \%$ & $100.0 \%$ \\
\hline
\end{tabular}

Fisher's Exact Test .003

C3 are the least likely to be happy with their living accommodation.

Gravidographic clusters (GCs) and Pregnancy Circumstance profiling

Table 5.137. GCs by Weeks aware of pregnancy

\begin{tabular}{llllll}
\hline Weeks & $\mathbf{C 1}$ & $\mathbf{C 2}$ & $\mathbf{C 3}$ & $\mathbf{C 4}$ & Total \\
\hline $0-6$ & 136 & 79 & 42 & 95 & 352 \\
& $95.1 \%$ & $86.8 \%$ & $56.0 \%$ & $70.9 \%$ & $79.5 \%$ \\
$7-12$ & 7 & 12 & 28 & 35 & 82 \\
& $4.9 \%$ & $13.2 \%$ & $37.3 \%$ & $26.1 \%$ & $18.5 \%$ \\
$13+$ & 0 & 0 & 5 & 4 & 9 \\
\multirow{2}{*}{ Total } & $0.0 \%$ & $0.0 \%$ & $6.7 \%$ & $3.0 \%$ & $2.0 \%$ \\
& 143 & 91 & 75 & 134 & 443 \\
& $100.0 \%$ & $100.0 \%$ & $100.0 \%$ & $100.0 \%$ & $100.0 \%$ \\
\hline
\end{tabular}

Fisher's Exact Test .000 
C3 members are less likely than other clusters to be aware of the pregnancy between 0-6 than other clusters. C1 members were almost all aware by 0-6 weeks.

Table 5.138. GCs by Original Intentions for Pregnancy

\begin{tabular}{llllll}
\hline $\begin{array}{l}\text { Considered } \\
\text { ending }\end{array}$ & $\mathbf{C 1}$ & $\mathbf{C 2}$ & $\mathbf{C 3}$ & $\mathbf{C 4}$ & Total \\
\hline Frequently & 3 & 2 & 12 & 5 & 22 \\
& $2.1 \%$ & $2.2 \%$ & $16.0 \%$ & $3.7 \%$ & $5.0 \%$ \\
Occasionally & 0 & 3 & 10 & 4 & 17 \\
& $0.0 \%$ & $3.3 \%$ & $13.3 \%$ & $3.0 \%$ & $3.8 \%$ \\
Only for a & 3 & 5 & 19 & 18 & 45 \\
moment & $2.1 \%$ & $5.5 \%$ & $25.3 \%$ & $13.4 \%$ & $10.2 \%$ \\
Never & 137 & 81 & 34 & 107 & 359 \\
& $95.8 \%$ & $89.0 \%$ & $45.3 \%$ & $79.9 \%$ & $81.0 \%$ \\
Total & 143 & 91 & 75 & 134 & 443 \\
& $100.0 \%$ & $100.0 \%$ & $100.0 \%$ & $100.0 \%$ & $100.0 \%$ \\
\hline
\end{tabular}

Fisher's Exact Test .000

C3 members were most likely to consider ending the pregnancy. C1 and C2 members were least likely to consider it, and most, never.

Table 5.139. GCs by Type of LMC care

\begin{tabular}{llllll}
\hline LMC & C1 & C2 & C3 & C4 & Total \\
\hline Midwife & 98 & 62 & 59 & 92 & 311 \\
Midwife/GP & $68.5 \%$ & $68.1 \%$ & $78.7 \%$ & $68.7 \%$ & $70.2 \%$ \\
& 4 & 6 & 9 & 12 & 31 \\
Midwife and private & 31 & 13 & 2 & 18 & 64 \\
obstetrician & $2.8 \%$ & $6.6 \%$ & $12.0 \%$ & $9.0 \%$ & $7.0 \%$ \\
Midwife and & 10 & 10 & $5.7 \%$ & $13.4 \%$ & $14.4 \%$ \\
hospital & & & 5 & 12 & 37 \\
obstetrician & $7.0 \%$ & $11.0 \%$ & $6.7 \%$ & $9.0 \%$ & $8.4 \%$ \\
Total & 143 & 91 & 75 & 134 & 443 \\
& $100.0 \%$ & $100.0 \%$ & $100.0 \%$ & $100.0 \%$ & $100.00 \%$ \\
\hline
\end{tabular}

Fisher's Exact Test .004

C1 is more likely to have a private obstetrician than any other cluster and C3, least. 
Table 5.140. GCs by Higher risk level

\begin{tabular}{llllll}
\hline Higher risk & C1 & C2 & C3 & C4 & Total \\
\hline No & 122 & 70 & 58 & 111 & 361 \\
Yes & $85.3 \%$ & $76.9 \%$ & $78.4 \%$ & $83.5 \%$ & $81.9 \%$ \\
& 19 & 19 & 8 & 14 & 60 \\
Not sure & $13.3 \%$ & $20.9 \%$ & $10.8 \%$ & $10.5 \%$ & $13.6 \%$ \\
& 2 & 2 & 8 & 8 & 20 \\
Total & $1.4 \%$ & $2.2 \%$ & $10.8 \%$ & $6.0 \%$ & $4.5 \%$ \\
& 143 & 91 & 74 & 133 & 441 \\
& $100.0 \%$ & $100.0 \%$ & $100.0 \%$ & $100.0 \%$ & $100.0 \%$ \\
\hline
\end{tabular}

Fisher's Exact test.013.

C2 has the greatest number of respondents who perceive themselves as higher risk. C3 has the greatest number of unsure.

\section{Fertility treatment}

Table 5.141. GCs by Ovulation/Fertility medication

\begin{tabular}{llllll}
\hline & C1 & C2 & C3 & C4 & Total \\
\hline No & 37 & 26 & 16 & 26 & 105 \\
& $74.0 \%$ & $92.9 \%$ & $100.0 \%$ & $92.9 \%$ & $86.1 \%$ \\
Yes & 13 & 2 & 0 & 2 & 17 \\
& $26.0 \%$ & $7.1 \%$ & $0.0 \%$ & $7.1 \%$ & $13.9 \%$ \\
Total & 50 & 28 & 16 & 28 & 122 \\
& $100.0 \%$ & $100.0 \%$ & $100.0 \%$ & $100.0 \%$ & $100.0 \%$
\end{tabular}

Fisher's Exact Test .020.

Table 5.142. GCs by Artificial insemination

\begin{tabular}{llllll}
\hline & C1 & C2 & C3 & C4 & Total \\
\hline No & 45 & 28 & 16 & 28 & 117 \\
& $90.0 \%$ & $100.0 \%$ & $100.0 \%$ & $100.0 \%$ & $95.9 \%$ \\
yes & 5 & 0 & 0 & 0 & 5 \\
& $10.0 \%$ & $0.0 \%$ & $0.0 \%$ & $0.0 \%$ & $4.1 \%$ \\
Total & 50 & 28 & 16 & 28 & 122 \\
& $100.0 \%$ & $100.0 \%$ & $100.0 \%$ & $100.0 \%$ & $100.0 \%$ \\
\hline
\end{tabular}

Fisher's Exact Test .091 
Table 5.143. GCs by IVF

\begin{tabular}{llllll}
\hline & C1 & C2 & C3 & C4 & Total \\
\hline No & 39 & 25 & 16 & 28 & 108 \\
& $78.0 \%$ & $89.3 \%$ & $100.0 \%$ & $100.0 \%$ & $88.5 \%$ \\
Yes & 11 & 3 & 0 & 0 & 14 \\
& $22.0 \%$ & $10.7 \%$ & $0.0 \%$ & $0.0 \%$ & $11.5 \%$ \\
Total & 50 & 28 & 16 & 28 & 122 \\
& $100.0 \%$ & $100.0 \%$ & $100.0 \%$ & $100.0 \%$ & $100.0 \%$ \\
\hline
\end{tabular}

Fisher's Exact Test .007.

As seen in the Tables above, for the 122 respondents who required one or more treatments to assistance to conceive, they almost all populate Cluster 1. 


\section{Gravidographic Clusters and Pre-existing values}

Profiling of the Gravidographic clusters and continuous variables was performed with ANOVA. Tukey's HSD was used to determine $p$ values and significance between clusters. Table 5.83 shows significant $(p \leq 0.05)$ df $(3,439)$ findings.

Table 5.144. GCs by Pre-existing influences

\begin{tabular}{|c|c|c|c|c|c|c|c|}
\hline Item/scale & C1 & $\mathbf{C 2}$ & C3 & C4 & $\mathbf{F}$ & $\mathbf{p}$ & Comment ( $95 \%$ confidence) \\
\hline \multicolumn{8}{|l|}{ Pre-existing influences } \\
\hline *Time orientation & 5.44 & 5.10 & 4.11 & 4.85 & 14.02 & .000 & $\begin{array}{l}\text { C3 has lower means than all other clusters. } \mathrm{C} 1 \text { is higher than } \mathrm{C} 3 \text { and } \\
\text { C4. }\end{array}$ \\
\hline *Seeks health information & 5.34 & 4.84 & 4.29 & 4.94 & 8.79 & .000 & $\begin{array}{l}\text { C3 is lower than all other clusters. C2 and } 4 \text { are similar. C1 is higher } \\
\text { than C2 and C3. C1 and C4 are similar. }\end{array}$ \\
\hline *Pre-pregnancy health & 5.67 & 5.09 & 4.41 & 5.33 & 29.54 & .000 & $\begin{array}{l}\mathrm{C} 1 \text { and } \mathrm{C} 4 \text { are similar. } \mathrm{C} 4 \text { is similar to } \mathrm{C} 2 . \mathrm{C} 3 \text { has the lowest mean } \\
\text { and does is significantly different from all other clusters. }\end{array}$ \\
\hline Environmentalism & 4.97 & 4.51 & 4.33 & 4.73 & 6.30 & .000 & $\mathrm{C} 1$ and $\mathrm{C} 4$ are similar. $\mathrm{C} 4$ is also similar to $\mathrm{C} 2$ and $\mathrm{C} 3$. \\
\hline Familism & 3.46 & 3.54 & 3.80 & 3.53 & 3.51 & .015 & $\begin{array}{l}\text { C1, C2 \& C4 are equal. C } 3 \text { is lowest and significantly different from all } \\
\text { other clusters. }\end{array}$ \\
\hline Sense of Mastery & 5.82 & 5.30 & 4.58 & 5.51 & 25.98 & .000 & $\begin{array}{l}\text { C3 is lower than all other clusters. } \mathrm{C} 2 \text { is similar to } \mathrm{C} 4 . \mathrm{C} 1 \text { is } \\
\text { significantly different to } \mathrm{C} 2 \text { and } \mathrm{C} 3 \text {. }\end{array}$ \\
\hline Trait anxiety & 3.42 & 3.86 & 3.99 & 3.58 & 3.30 & .021 & There are two subsets: $\mathrm{C} 1,2$ and $\mathrm{C} 4$ and then $\mathrm{C} 4, \mathrm{C} 2$ and $\mathrm{C} 3$. \\
\hline \multicolumn{8}{|l|}{ Pregnancy circumstance } \\
\hline Rating as a life event & 8.88 & 8.03 & 7.24 & 8.90 & 34.48 & .000 & $\mathrm{C} 3$ rates the pregnancy the least as a positive life event. \\
\hline Feeling better than usual & 3.23 & 2.16 & 2.75 & 3.01 & 10.01 & .000 & C1 and C4 are more likely to 'feel better than they normally do'. \\
\hline \multicolumn{8}{|l|}{ Information } \\
\hline Item/scale & $\mathbf{C 1}$ & $\mathbf{C 2}$ & C3 & C4 & $\mathbf{F}$ & $\mathbf{p}$ & Comment ( $95 \%$ confidence) \\
\hline Information source partner & 4.12 & 3.03 & 3.69 & 4.30 & 7.45 & .000 & $\mathrm{C} 2$ and $\mathrm{C} 3$ regard their partner less useful than $\mathrm{C} 1$ and $\mathrm{C} 4$. \\
\hline Information source mother & 4.43 & 4.53 & 5.48 & 4.86 & 5.431 & .001 & C3 see their mother as useful significantly higher \\
\hline
\end{tabular}




\begin{tabular}{|c|c|c|c|c|c|c|c|}
\hline Item/scale & C1 & $\mathbf{C 2}$ & $\mathbf{C 3}$ & $\mathbf{C 4}$ & $\mathbf{F}$ & $\mathbf{p}$ & Comment ( $95 \%$ confidence) \\
\hline$*$ Interest in change after info & 1.76 & 2.20 & 2.83 & 1.94 & 13.17 & .000 & C3 has the least interest in changing. \\
\hline *Information change result & 5.48 & 5.25 & 4.93 & 5.61 & 7.76 & .000 & $\mathrm{C} 2$ and $\mathrm{C} 3$ felt they changed the least as a result of information. \\
\hline \multicolumn{8}{|l|}{ Pregnancy attitudes } \\
\hline Need a Dr also & 3.48 & 3.87 & 4.09 & 3.62 & 2.86 & .037 & C3 has the highest belief in needing a Doctor. \\
\hline Technology makes birth safer & 5.76 & 5.90 & 5.32 & 5.75 & 3.43 & .017 & All have high means. $\mathrm{C} 2$ the highest. \\
\hline $\begin{array}{l}\text { Importance of accomplishing } \\
\text { goals }\end{array}$ & 5.03 & 4.55 & 4.99 & 5.22 & 5.49 & .001 & $\mathrm{C} 1$ and $\mathrm{C} 4$ most goal driven. \\
\hline Avoids medication & 5.76 & 5.90 & 5.32 & 5.75 & 4.16 & .006 & C3 had slightly lower mean for avoiding medication \\
\hline Takes OTC meds & 4.27 & 4.97 & 4.37 & 4.37 & 2.83 & .038 & Similar tolerance for taking OTC drugs. C1 the lowest. \\
\hline $\begin{array}{l}\text { Good to know baby's sex before } \\
\text { birth }\end{array}$ & 3.13 & 3.47 & 4.08 & 3.51 & 4.89 & .002 & C3 has the highest mean. \\
\hline $\begin{array}{l}\text { *Pregnant women shouldn't } \\
\text { smoke }\end{array}$ & 6.76 & 6.76 & 6.01 & 6.72 & 10.92 & .000 & C3 had least strong attitudes. Different from other clusters. \\
\hline *Others shouldn't smoke & 6.40 & 5.91 & 5.08 & 6.01 & 14.22 & .000 & C3 had least strong attitudes. Different from other clusters. \\
\hline *Shouldn't have alcohol & 6.39 & 5.52 & 6.36 & 6.41 & 10.11 & .000 & C2 had less strong attitudes. Different from other clusters. \\
\hline Psycho-social & & & & & & & \\
\hline $\begin{array}{l}\text { *Support importance from } \\
\text { partner }\end{array}$ & 6.93 & 6.67 & 5.78 & 6.66 & 10.52 & .000 & C3 the lowest. \\
\hline Support importance from mother & 5.29 & 5.71 & 6.05 & 5.76 & 3.31 & .020 & $\mathrm{C} 1$ lowest and $\mathrm{C} 3$ the highest. \\
\hline
\end{tabular}


Table 5.145. Gravidographic Clusters and Non-significant Cross-tabulations and ANOVAs

\begin{tabular}{|c|c|c|c|}
\hline Item & $\mathbf{p}$ & Item & $\mathbf{p}$ \\
\hline Pregnancy circumstance & & Pre-existing influences & \\
\hline Previously pregnant* & .344 & Cultural Strength & .363 \\
\hline Gestational group & .731 & Health as a Value & .606 \\
\hline Knows sex of baby & .220 & Health Consumerism & .094 \\
\hline Locality of birth** & .655 & Attitude to Change & .471 \\
\hline Used to find LMC & & Materialism & .432 \\
\hline Personal recommendation & .499 & Religiosity & .268 \\
\hline Pamphlet & .822 & Superstition & .703 \\
\hline 0800 number & .862 & Concern for physical appearance & .217 \\
\hline Info on website & .146 & & \\
\hline Through GP's rooms & .104 & & \\
\hline Telephone contact list & .656 & & \\
\hline Interviews & .576 & & \\
\hline
\end{tabular}

*Evenly distributed in clusters (including younger age groups)

** Homebirth women are not confined to any particular cluster.

The above non-significant findings point to a lack of difference in means between the clusters for these variables. 


\subsubsection{Description of Gravidographic clusters by profiling variables}

The Gravidographic clusters are further described by results from profiling below.

C1 'Planners and Pursuers of Health' is made up of those who have higher education and income. Demographically members are very similar to C2. They have the largest number of those who required fertility treatment and are aware of their pregnancy early. They have the largest number of those engaging private obstetricians. $\mathrm{C} 1$ is very different from $\mathrm{C} 3$ but similar in many respects to $\mathrm{C} 2$ and $\mathrm{C} 3$.

C2, 'Mixed Responders' is made up of more members with a high risk pregnancy than other clusters. $\mathrm{C} 2$ is similar to $\mathrm{C} 1$ in regard to income, education and age. These have a more tolerant attitude toward alcohol during pregnancy than other clusters.

C3, 'Lesser Attention to Health' is made up of those least resourced to care for their health. They are younger, have less income and less educational qualifications. They tend to rely more on their mothers for support and information than other clusters. They also use the Internet less than other clusters. Māori and Pacific women are most likely to be in C3 or C4. C3 members are likely to live in a small town or city than those in $\mathrm{C} 1$ and $\mathrm{C} 2$.

C4, 'Slow Starters but Catch Up' has the next youngest age profile after C3. It has a higher number of those living in a small town (similar to C3) when compared to $\mathrm{C} 1$ and $\mathrm{C} 2$. Educational qualifications are less than $\mathrm{C} 1$ and similar to C2 (but higher than C3). Overall, household income is lower than C1 and C2. Those specifying factory and trade work make up $40 \%$ of C4 members. C4 health attitude means are similar to $\mathrm{C} 1$ and $\mathrm{C} 2$.

\subsection{SUMMARY OF CLUSTERING PHASE}

This draws to a close the sub-section on clustering. The clustering has led to the identification of four useful pregnancy health lifestyle segments that met the criteria of distinctiveness, parsimony and being non-sparse. This also ends the quantitative section of the results. 


\subsection{CHAPTER 5 SUMMARY}

A comprehensive analysis of the data has been reported in this chapter. Descriptive analysis and bi-variate tests of association were conducted first. This assisted in the formation of sub-clusters that reduced data and also led to insights regarding variables to put forward into the Gravidiographic clustering procedure. The Gravidographic clustering led to four health lifestyle segments that were profiled to allow their characteristics to be better understood. The study discussion, recommendations and closing remarks follow in Chapter 6 . 


\section{CHAPTER 6. DISCUSSION AND CONCLUSION}

\subsection{INTRODUCTION}

This chapter contains the discussion of findings and concluding sections of the thesis. The discussion of findings is presented in sections and follows the ordering of the eight research questions the study addressed.

Section 6.2 discusses the health lifestyle of the nulliparous market overall from the insights that have been drawn from the analysis of the descriptive data (Research Objective 1). Then, the lifestyle segments that were found, and their characteristics (Research Objective 2) are discussed. The potential reasons why they may have formed as they did are also examined. Next, the concept of successful transition is discussed in light of the findings and whether changes made in the pregnancy lifestyle could be considered part of a trajectory or illustrative of a more profound and permanent change (Research Objective 3). Finally, remarks regarding the conceptual framework are made as a result of consideration of the study findings.

The chapter ends with a discussion of limitations (Section 6.3), recommendations for future research (Section 6.4), theoretical contribution and managerial/practice recommendations (Section 6.5) and concluding author comments (Section 6.6).

\subsection{DISCUSSION OF FINDINGS}

\subsubsection{A DESCRIPTION Of THE HEALTH LIFESTYLE OF THE PREGNANT NULLIPAROUS MARKET}

A key objective of the study was to provide a comprehensive description that would lead to a better understanding of this market in regard to health lifestyle during a transition into a new life stage (Full Nest 1), as defined by research Question One. As will be discussed, many of the findings support the extant literature reported in Chapter 2, whilst some new evidence emerges that adds further insight into this group of women. The overall goal of this study was to comprehensively describe and segment the health lifestyle of pregnant women in a holistic manner, rather than expand any one particular attitude or behaviour in detail, at the expense of another. There is much discussion that could be had 
over any one area in particular, but the level of discussion applied is limited to that which meets the research objectives and questions for the study.

A summary discussion of the descriptive and bi-variate analyses is given below, presented according to the conceptual framework. A graphic presentation of the conceptual framework is given at the beginning of each sub-section of Section 6.2 with the sub-domains discussed in that part shaded. This section addresses Research Question 1: "How can the pregnant nulliparous market be described in regard to collective values, attitudes, health practices and additional factors that make up the pregnant health lifestyle?

\subsubsection{Influencing Lifestyle Factors}

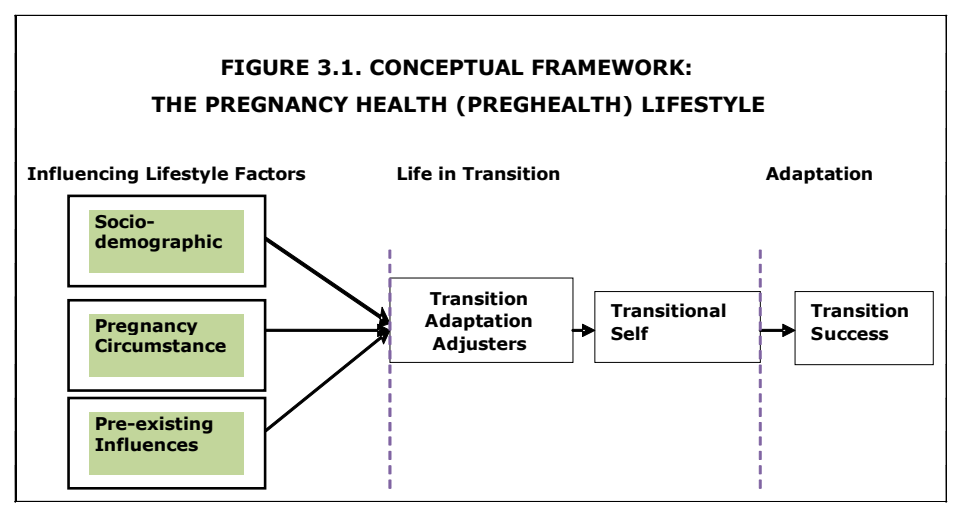

\section{Socio-Demographic}

A significant amount has already been said about the demographics of the respondent group and will be further discussed in latter stages of this chapter. Demographics play a key role in health events and pregnancy lifestyle and supports findings of other studies (Chomitz, Cheung, \& Lieberman, 1995) that certain health lifestyle predispositions can be linked to factors such as education, ethnicity and income. However, the intertwining of demographics and health is complex and the findings of this study give credence to the usefulness of studies that include psychographic variables to enrich understanding of why these women hold the attitudes and behave as they do.

\section{Pre-existing influences.}

This sub-domain comprised of two main constructs (pre-existing health and mastery and achievement), along with an attribute set of eleven other traits and values. In this study, understanding pre-existing influences and how they impact on pregnancy health choices and behaviours were explored, as value based 
variables are theoretically more enduring and therefore would remain relatively consistent through pregnancy.

Collectively the women viewed general health as important and are positively involved in their health. In caring for their general health, 'time put in' is considered one of the least active influences, followed by their assessment of overall fitness. The women display high levels of health consumerism, being happy to pursue information and information, but a little less happy to challenge a health professional. Health involvement may be influenced by the age of the cohort (having grown up in a period of greater societal orientation to health services as a consumer product and increased media profiling of health issues). Additionally, given that many women had a pregnancy wish or intention, they may have been in a more health conscious space. Comparison between prepregnancy health level and intendedness of the pregnancy is interesting, with 'wanted' and 'wanted earlier' intentions showing the highest manifest prepregnancy health level scores, and 'mistimed' and 'unwanted' the lowest. Measures of factors such as mastery, motivation and effort and efficacy for change could be considered high overall and were also positively associated with health performance.

The findings provide a picture of women having a moderate degree of concern for their physical appearance, with respondents on average being somewhat concerned about their appearance (although probably not unusual in a young female demographic). A 'concern for physical appearance' pre-pregnancy was not associated with low levels of body satisfaction in pregnancy and most women enjoy experiencing their pregnant body. However, in pregnancy, a low level of body satisfaction and/or a high level concern over weight could well be an indicator of disruptions to transition success.

Levels of environmentalism were generally higher than for materialism; that is, these women tend to see sustainability as more important than gaining possessions. The study demonstrated similar findings to other studies with attitude toward environmentalism being higher than behavioural intent. Overall the group was not religious, in fact, many marked within the disagreement range of responses, and thirty percent in strong disagreement. Interestingly, superstition was higher than religiosity, although approximately seventy percent did not regard themselves as superstitious. The importance of cultural beliefs and practices features more highly than religiosity. 
'Time orientation' has been proven to be linked to health in previous studies (Hornik, 1990). Time orientation was moderately strong with most indicating a preference to plan ahead. A future time orientation was positively correlated with a higher pre-pregnancy health level, consistent with the findings of Hornik (1990). There was a statistically significant difference between smokers and nonsmokers, with non-smokers showing a higher future time orientation.

In regard to 'Universal values', for the group, self respect, security and relationships scored the most highly. This may well be related to their current developmental stage, where pregnancy brings about increased focus on relationships and nurturance. Fun and enjoyment were also valued highly. Again the findings may be reflective of women in their twenties and thirties, rather than from a sample of older people. 'Excitement' had a weak positive correlation to Pre-pregnancy Health Level, so did not support the findings of authors, such as Divine and Lepisto (2005) who found a high level of excitement was correlated negatively to an individual's health pursuit. However, as this group is considered young and healthy, it is not untoward that they enjoy a moderate amount of 'excitement' without it impacting on health.

Women's Role items showed little variance in regard to 'right to be as independent as they want', 'can achieve anything they want' and 'balance between a career and children is an important issue for women', demonstrating what the women collectively felt similarly and strongly about. A more varied response was found for the two items: 'having children is the most important role for women' and 'women should have a career and children', showing a degree of division amongst the women. The value 'Familism' did not score highly in the study, despite women demanding a high degree of emotional support in the pregnancy transitional self findings. It may mean that there is egocentricity associated with pregnancy in regard to familial nurturance and obligations. However, this must be tempered by the fact that the Familism scale has in most recent times been studied and focused within the Latino culture (Villarreal et al., 2005; Luna et al., 1996) and therefore is exploratory in this context. In this study, 'Familism' was highest for Asian respondents. This could be related to strong cultural family affiliation or an influence of the importance of family networks staying together in a country with a different culture. Two new items that did score highly and were associated with the idea of family were 'families can live apart and still be close' (representing distributed family networks and 
separation by distance and communication through technology) and 'friends can be more like family than blood relatives'.

In regard to media usage, television had an almost $100 \%$ saturation. A high use of the Internet overall reveals most women have the ability to access web-based material. It appears that women are highly likely to read printed information if it were given to them in pregnancy, with a high degree of pamphlet readership shown. Newspaper readership was also relatively high. Business tabloids were not highly read and any uptake was predominantly in professional groups. The rate of readership for women's magazines and lifestyle magazines was surprisingly low, given the quantity and range of subject matter available that is targeted to younger women (for example, fashion magazines). Magazines are not as widely read as the newspaper and community papers. As discussed previously, the meaning of the high score for direct mail could not be interpreted with a great degree of surety as to the meaning women made of the question. PlayStation or Internet games are not rated highly and are more popular in the younger age group.

Trait Anxiety proved to have a positive correlation with the level of anxiety felt during the pregnancy period, thus supporting previous findings by authors such as Spielberger (1983), where a strong trait anxiety person would expect to become more anxious in a certain situation than a low anxiety person. Trait anxiety also has associations with other variable that were anxiety or concern based (for instance, weight concern).

\section{Pregnancy Circumstance}

Many women with mistimed pregnancies performed well in terms of transition and care with pregnancy health, when compared with those with a pregnancy intention. This demonstrates the capacity of women to accommodate changes that may be needed despite the pregnancy being unintended. Those whose pregnancy was rated as unwanted were the smallest group but consistently performed less well for health promoting attitudes and behaviours of all other pregnancy intention groups (including mistimed). This further supports other authors' suggestions, such as Speizer, et al., (2004), D'Angelo, et al., (2004) and Altfeld, et al., (1997), that 'planned' versus 'unplanned' is too simple a categorisation for the pregnancy event, in regard to how they will adjust to pregnancy. Eighty percent of women never considered ending the pregnancy in the initial few weeks, even when the overall early awareness of pregnancy 
(average of 6 weeks) gave them a good degree of time in which to decide if they wanted to terminate the pregnancy. This was further supported by their rating of the pregnancy as a life event, where the majority of women scored it very highly as a positive life event, with many rating it as the best event that had happened to them. There is little difference between intendedness of pregnancy and 'feelings now', except for the 'did not want' group, who are distinctly different from all other groups. Again this demonstrates that most women accommodate their pregnancy well into their life course whether planned or unplanned. However, care must be taken with this attitude, as those who had made a decision for a termination were not included in this study.

With almost all the women knowing they were pregnant early in the gestational period, (most likely through the availability of relatively reliable pharmacy or supermarket self diagnosis kits), a degree of certainty regarding their pregnant status is obtained at an early stage. Women also have control over how frequently they test for pregnancy (after cost considerations), and this marks a change from when women needed to await 'official' diagnosis from a medical practitioner. This diagnostic ability, however, means that at an early stage, a woman has potentially a significant period of self-responsibility for health decisions before establishing regular contact with their LMC and at a critical time of the pregnancy in regard to fetal development. An important finding from the questionnaire was the period post-diagnosis that, for some, required a significant amount of effort and stress to engage a carer.

There is variety in the availability of information regarding how to obtain a carer and in the difficulty experienced obtaining one. There was no relationship found with any variable tested against it, except region of domicile. Of those who found it 'difficult to find an unbooked midwife' (about a third), it seems to be more common in cities rather than smaller towns. Half of the women found they 'did not have a lot of choice', although most felt comfortable with their carer, once obtained. Some women recorded experiences with finding a midwife in a final additional comments section provided at the end of the questionnaire. Indeed for some, the experience was very distressing for the woman concerned. Although the decision process in procuring an LMC was not a major part of this study, the importance of personal recommendation for approach to an LMC is noteworthy with almost half using recommendation solely or in combination with other factors. The significance of personal recommendation may well be a reflection of the high level of credence, as well as current market conditions with midwives 
tending to obtain bookings without the need to actively promote their services. As it is the first birth, a lack of experience or previous existing relationship is also significant. At the present time, positive word of mouth from previous clients is perhaps the highest and most effective form of communication for LMCs wanting to promote their services. Advertising and telephone books were not used a great deal.

Collectively, seventy percent of LMC care women engaged was 'midwife only' (which equated with reported figures from the Report on Maternity (Ministry of Health, 2004), demonstrating the high level of primary maternity care in New Zealand that does not involve a doctor. This further exemplifies the potential problem of studies related to pregnancy and birth in New Zealand using measurement tools transferred from health studies where doctor led medical care (and potentially a disease orientation) is the predominant model. Choice of private specialist was correlated with higher age, probably being related to available resources (with private obstetric care being fee-based) and/or a higher incidence or perception of high risk and acknowledgment of a potential biological disadvantage that accompanies increased age.

\subsubsection{Transition Adaptation Adjusters}

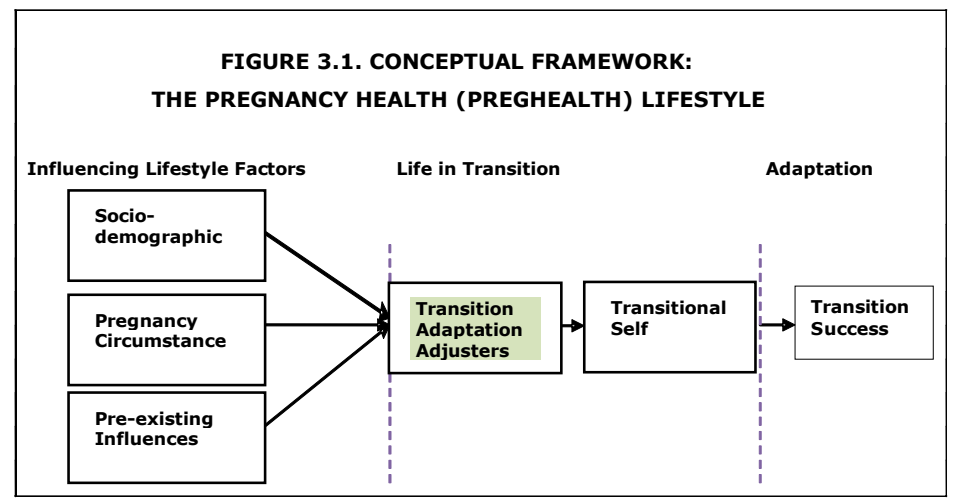

Information and attitudes formation were explored as the two key transition adjusters. Information seeking and use by women, in regard to pregnancy related information, was high and there is no doubt that overall pregnant women were active seekers of information. The women sought information based on positive outcome beliefs: that it increased confidence, helped in decision making and reduced anxiety. There was a very strong sense of wanting to be involved in decisions with almost all wanting to know if 'something is wrong'. Whilst most felt they could cope with a lot of information and felt able to understand and 
process it, there was recognition that information received was often conflicting. 'Ways of knowing' demonstrated that women do not regard medical information as the only legitimate and useful way of gaining knowledge. For instance, in this group of women, balancing information with 'intuition' was important. This finding supported the work of other authors, such as Erdelez (1999).

For most, information received led to adjustment of attitudes and practices if indicated. The LMC was regarded as the most useful source of information, along with scientific type tests. The partner was a low source of useful information for most. Attendance at a formal series of classes is undertaken by most women, with a range of topics seen as important. The role of the classes as a way of involving the father of the baby was regarded as just as important as topics related to labour, birth and caring for a baby. 'Meeting others' was also seen as very beneficial, highlighting that antenatal classes perform a social role that is regarded, like content, an important benefit.

For pregnancy specific health attitudes, the overall picture of women was clear as being one of strong self-efficacy and a wish to be involved in decisions about the pregnancy. The availability of products that enable women to prove their pregnant state themselves (for example, supermarket and pharmacy purchase urine tests) has given them autonomy previous generations of women have not had, and this sample of women showed little resistance to using them.

In regard to attitudes to specific health behaviours, woven through the study were groupings of attitudinal measurement scales and items related to specific health behaviours of dimensions. These included substances, exercise, nutrition, and weight gain. These attitudes are discussed in conjunction with the specific health behaviour they relate to.

There was high agreement that pregnancy was a natural event, yet in contrast, the acceptance of 'technology' as an aid was useful and trusted more than 'leaving it to nature'. This was particularly seen in the uptake of a scan (given that it is recommended but can be declined). The strongest reasons for agreeing to a scan were to see if the baby was 'OK' (not leaving it to nature) and to date the pregnancy. Other reasons, not the most important, but still regarded as important were those concerned with validation of the 'real' baby; getting in touch with the baby', 'see it is real', and 'to get the baby's father involved' were 
genuinely thought to be facilitated by a scan and similar to the findings of Taylor (2000).

There appears to be a comfort with and acceptance of technology by the women, rather than regarding it as an unnecessary intervention. Technology acceptance in pregnancy may be a feature of this cohort more than their historic predecessors, but does not bode well for the 'non-intervention' principles of natural childbirth. Attitude to knowing the sex of the baby was approximately fifty percent divided and seems to equate with the level of women in the survey who acknowledged that they knew the sex of their baby. It was initially thought that the technology items would form a satisfactory scale for a 'Technology in Pregnancy Health Locus of Control', but this proved not to be the case. Although a new subscale was not achieved in this study, it still offers a fruitful area of future inquiry, particularly as the next childbearing cohort will have been raised in a technology rich environment.

As discussed in Chapter 5, the Health Locus of Control (Wallston et al., 1978), with its three subscales, did not perform well in this particular context. It is interesting that for such a motivated group of women, who reported a high level of efficacy and mastery, the Internal Locus of Control score was lower than for some Powerful Other items. Pregnancy is not a disease process nor purely is it a health promotion and/or maintenance context. As a specific state of being, the findings do suggest that its measurement requires customised scales to measure Locus of Control type beliefs. In New Zealand, the nature of the partnership model may also be confounding the performance of scales such as the Health Locus of Control and explain the cross loading of some items that might not be seen in other health practitioner-patient relationship studies. The finding that many women believe an 'unhealthy lifestyle might still produce a healthy baby' (retained as a single item) is of interest and seems to conflict with the level of pursuit women engage in to protect and nurture their baby. It may suggest that there are some aspects of fetal endowment or inheritance that to them remain outside health provider or internal control.

Levels of motivations and influences for change are high within pregnant women. Over a third regarded achieving goals as very important. Reasons for efforts to maintain health or make changes were highly motivated by self-oriented concern overall. However, the most consistent and highest driver of decisions and action were on behalf of the baby and a desire to do the best for the baby. This finding 
is consistent with other authors, who have found that the welfare of the baby is a strong motivation for moving to protect and nurture the baby during pregnancy (Rubin, 1975; Mercer, 2004). Time was the largest barrier encountered to maximising health and for most, 'lack of time' was perceived as a greater barrier than factors such as transport and costs.

\subsubsection{The Transitional Self}

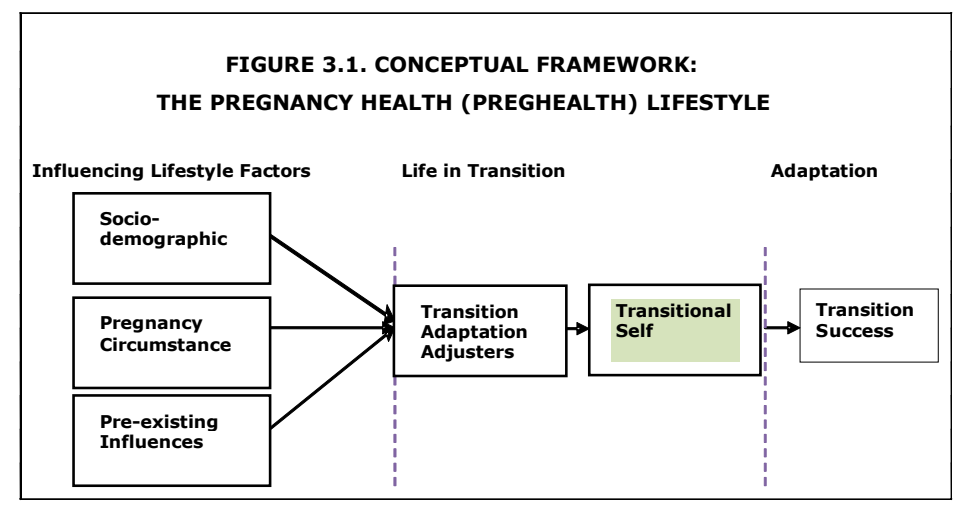

Most women made a significant effort to maintain or establish good health behaviours and support across both the physical and psycho-social sub-domains. One finding that held consistently across most areas is that attitudinal indicators were generally slightly higher than the corresponding behaviour indicator. For example, positive attitudes related to the value and importance of good nutrition had a higher mean than actual nutrition performance itself. This is not uncommon in the literature, whereby intent to perform a certain 'positive' behaviour is generally higher that the actual behaviour itself. The finding that attitudes scored higher that actual practice rep is not an unusual finding, where marketing literature cautions that an expression of a positive attitudinal 'purchase intent' is not an assurance of behaviour.

\section{Nutrition}

Overall, a high degree of care with nutrition was demonstrated by the women. The least care appeared to be in avoiding 'junk food'. Conversely, 'risky' food, that which is considered dangerous to the fetus because of the risk of disease, was largely avoided and was a major influence over eating patterns and for many presented quite some difficulty in accommodating it into daily living activities. The high avoidance of risky food was consistently an indication of the effort women undertook to ensure their baby experienced a risk free environment. For eating pattern disturbances, morning sickness and changes in food preferences had a 
higher incidence than developing cravings. However, there was a wide variability in these findings.

Weight gain in pregnancy is a normal event and too little weight gain can be a sign of intra-uterine growth retardation, or the woman eating less than nutritional requirements. At the same time, weight gain and loss of the 'ideal' feminine body shape can be concerning for some women. In this study, variables for nutrition, related to health and growth of the pregnancy, were assessed in the health behaviour set of measures. Variables for feelings about weight gain and body image and concern over weight gain were assigned to the psycho-social domain. Pregnant women overall felt they knew how to eat healthily and cared about their nutrition. There was a high belief that good nutrition increases the chance of a healthy baby. Barriers to good nutrition were low overall, although again, time pressure was more likely to inhibit good nutrition. The findings also indicate that those who care for their health most pre-pregnancy will show the highest care for their nutrition in pregnancy. The positive link between pre-pregnant health maintenance and care of health in pregnancy is not an unusual finding as it has been found by several other authors.

The 'Folic acid profile' revealed that less than half of the women took folic acid prior to pregnancy, the most ideal time, compared to a little over half who 'did not take' it or took it 'after pregnancy confirmation'. This is consistent with concerns in the literature that many pregnant women are not taking folic acid at the optimal time (Green, 2002; De Walle \& de Jong-van de Berg, 2008). When compared with 'intendedness" of the pregnancy, it showed, not surprisingly, a positive association, as those who were planning to become pregnant would be in a better position to commence folic acid prenatally. However, of those women who stated they had a pregnancy wish, a third did not take folic acid until after confirmation. The reason for this is unknown, but shows a potential breakdown in health communication messages, where women are compliant with taking vitamin supplementation to reduce the risk of neural tube defects, but are mistiming optimal commencement.

\section{Exercise and rest}

Women strongly believe that exercise in pregnancy is good for their health and most do not believe it is dangerous for the baby. However barriers did negatively impact on exercise activity. In particular, these included being 'too tired', 'feeling unwell' and experiencing a lack of time, consistent with the findings of authors 
such as Clarke and Gross (2004). Women generally found that a lack of information about exercise during pregnancy did not cause a significant issue; the actual following of exercise recommendations showed more variation. Most women did not have a formal exercise plan or programme. Of the eighteen percent of women who had a plan, they were more commonly in the group of high exercisers, indicating that a formal plan might be a helpful pathway to meeting exercise requirements. By far the most commonly cited form of exercise was walking (over ninety percent). However, it is not known whether respondents included walking to accomplish daily activities of living as opposed to intentional walking for exercise in their responses. It would seem that walking is one of the most accessible forms of exercise for pregnant women. The next most significant form of exercise was swimming or aquarobics (twenty five percent).

Without a doubt, these women perceived the need for more rest during the pregnancy. Sleep pattern disturbance was very common; feeling tired was an overall feature of this group and appeared to pervade much of the pregnancy irrespective of gestation. It is difficult to know whether this is a feature of contemporary pregnancy, where women generally work fulltime well into the pregnancy. Comparing fatigue levels in women who had given up work against those who were working would also be spurious, as tiredness generally increases as gestation progresses, corresponding also with cessation of work.

\section{Risk reduction}

Risk reduction behaviour was assessed at several points in the questionnaire. Behaviours such as folic acid intake and avoiding risky food, whilst allocated to nutrition behaviour, are also risk reducing. Other behaviours that were used in the health study to measure risk reduction were attention to dental hygiene, wearing a safety belt in the car (in order to reduce environmental risk) and the uptake of an ultra-sound scan.

It is important in pregnancy to care for teeth and gums, particularly as women are at risk for gum disease due to the effects off oestrogen. Given the importance of dental hygiene in pregnancy, only approximately forty percent were in strong agreement that they were taking care of their teeth and gums. Dental hygiene in pregnancy appears to be being overlooked by women, as raised in the literature by authors such as Lydon-Rochelle, Krakowiak, Hujoel and Peters (2004). Whether it is a factor of the relatively high cost of dental care and/or a lack of knowledge about why it is important in pregnancy is not known. Car 
safety belt wearing is often used as a means of assessing care with environmental risk reduction in health promoting lifestyle studies. 'Always wearing a safety belt in a car' had a very high level of compliance overall. However, this could be reflective of the legal requirement in New Zealand rather than a specific choice.

The universal uptake of ultrasound scanning and the level of surveillance the technology provides could also be seen as another major indicator of the need for women to be assured that the fetus was safe and that the pregnancy could be expected to progress normally. Ultrasound scanning provides a combination of benefits, both medical and those that could be regarded as 'social'. Although approximately half of the women did not know the sex of their baby (and most would have had the opportunity), scanning was important for the women as a means by which they could network their baby to the outside world (particularly the baby's father). The findings of this study are similar to findings by authors, such as Taylor (2000), who have investigated the phenomenon of scanning, particularly investigating the benefits to the mother. As childbirth pregnancy technologies become increasingly sophisticated in the range of benefits and fetal 'gaze' ability they offer both providers and their patients, this will become an increasingly interesting research area in regard to the reasons women undertake ultrasound scanning.

\section{Substances}

As a collective respondent group, attitudes toward smoking in pregnancy were highly negative overall. Smokers had slightly less positive responses for nonsmoking in pregnancy than non-smokers and did show a little more tolerance toward 'others smoking' around pregnant women also. Given the education and mass media campaigns aimed at young adults over the past thirty or so years, it is of note that almost thirty percent of women were smoking at the onset of pregnancy and/or had been in the twelve months prior to becoming pregnant. It is a little more reassuring that of those women smoking, less than ten percent continued to smoke cigarettes during the pregnancy, with the greater percentage who continued to smoke cutting down as opposed to increasing.

When all smokers were asked what influenced their smoking behaviour in the pregnancy, by far the greatest influence was not wishing to harm the baby (consistent with the literature). Not wishing to harm themselves drew a much less strong response. Pressure from family and friends was more significant than the LMC (although it was not determined from the item whether the doctor or 
midwife had actually addressed the issue with them, or if it had been and had not been influential). The influence of health professionals was also lower than information in brochures and advertisements.

Over recent years there has been significant health promotion messages aimed at pregnant women to avoid any alcohol in pregnancy, because of the risk of fetal alcohol syndrome. Current New Zealand Ministry of Health guidelines (2006) recommend complete abstinence, although it is fair to say internationally there is controversy regarding exactly how much alcohol in pregnancy is dangerous for the baby and guidelines do vary between countries. Seventeen percent of women continued to drink alcohol during the pregnancy, although of these women, all reported cutting down and no one had increased consumption.

There is a positive correlation between continuing to consume alcohol and an unwanted pregnancy, but no significance was found with other intendedness of pregnancy categories, or educational status. However, statistical significance was found in the different age groups. Those in the 35-39 year age group had the highest propensity to continue drinking. In fact of those in this group, who were drinking before pregnancy $(n=64)$, half continued drinking. The next closest group for continuance was in the 30-34 age group. Whilst it seems that higher maternal age and drinking continuance are linked, this study cannot comment on why women continue to drink, particularly those from a higher education group. It is unlikely they have not heard of the risks attached to alcohol in pregnancy. It may be their interpretation of safe consumption is in dispute with New Zealand guidelines. The findings of Root and Browner (2004) may go some way to explain it. These authors found that women intentionally fuse 'a range of information by applying their own compliance and resistance to bio-medically derived prenatal norms' all the different information they obtain. There is also a statistical significance between smoking (both ceasing at pregnancy and continuing) and continuing to drink alcohol during pregnancy. Interestingly, there were a few women who indicated that they had ceased alcohol, but then also gave an indication of actual intake. It is not clear if this was a mistake in the comprehension of the questions, or for a few women, 'cessation' did not mean complete abstinence. A study by Morris, Swasy and Mazis (1994) supports these findings also, where they found drinking in an older demographic and a more sophisticated approach to "rationalising of understanding the risks to the unborn child" (p. 142). These authors also found that many of the women thought that a little alcohol was 'not drinking', as was possible in the responses in this study. 
Over seventy-five percent of the women were taking vitamin supplements. It was intended that this question extract the number of women taking vitamins in addition to folic acid, but in hindsight, it is not known whether women answered this question in response to their folic acid intake. In order to improve this, it would have been better to exclude folic acid to get a more accurate idea of supplementation outside of folic acid. Well over half of the women took 'OverThe-Counter' medication and almost a quarter were taking homeopathic remedies. The figure for homeopathic intake is not inconsistent with the attitudes to homeopathy findings. Many women fell into the neither agree/disagree domain for homeopathy, indicating some ambivalence toward it. It may be that if it were offered by the LMC, women would be receptive. Those attending doctors have less strong positive attitudes toward it. This finding is probably not unusual, given that positive views toward homeopathy are generally more strongly held by midwives than doctors (Gaffney \& Smith, 2004) and so therefore would be more likely to be exposed recommendation of homeopathy.

Few women declared the use of illegal drugs: marijuana, $(n=12)$ and party pills (not illegal at the time of data collection), $(n=6)$. It is not known if this is a valid reflection or not, as there may have been respondent sensitivity to giving a true response, or those taking these drugs may not have responded to the questionnaire.

\section{Emotions, stress and support}

For most women, the desired support, and the level to which it was thought to be received, was balanced. The most support was expected from the father of the baby, although for some, the woman's mother was a more significant support person. In particular, a mother as the most significant support was associated with younger age. For most, however, friends and family were equal to the mother in regard to overall levels of support sought and experienced. Pregnancy may also be an important time for daughters to hear from their mother the pregnancy and birth stories of their older female family members. A group often offered in the 'other' category were workmates. Given the large amount of time a nulliparous woman experiences in the workplace it does highlight the work environment as an important space for psycho-social nurturance.

The type of support desired by women was classified into three broad groups: pregnancy related (emotional care and enjoyment of life), functional assistance 
(helping with household chores) and financial support. All the categories ranked highly and in fact no one category was perceived as more desirous than the other, pointing to a wide range of support being sought. Although many women felt they were more emotional in pregnancy, they regarded themselves as still able to problem solve rationally rather than becoming 'emotional during a problem solving situation'.

Women reported their anxiety level in pregnancy at generally low rates. Findings showed that if 'Pregnancy State Anxiety' was elevated, so too was 'Life situation state anxiety'. 'Pregnancy State Anxiety' was a little higher than 'Life situation state anxiety' and was significantly different. Findings also showed that those with higher levels of trait anxiety would experience higher levels of pregnancy related anxiety than those women who tended not to be so anxious nonpregnant, findings that are is consistent with Spielberger, 1983, who linked a person with a high trait anxiety likely to demonstrate high state anxiety levels. Women engage in multiple activities to rest and relax in pregnancy. The most common is talking with family and friends, reading and watching television.

Worry over weight gain in pregnancy generally was low and corresponding pregnant body image was high. Of interest is the relationship between a positive 'Pregnancy Body Image' and 'Attachment'. Those who worried more about their weight also showed a strong tendency to have a lower level of positive pregnancy body image. This highlights that those women who are affected by reliance on weight control to maintain a positive body image non-pregnant could well be at risk for an assault on their self-image during pregnancy due to the inevitable weight gain that comes with pregnancy. The study findings also support the work of other authors (Müller, 1994; Priel \& Besser, 2001).

Increasingly, the interest in the popular press on celebrities has been noted. As many popular celebrities are in the child-bearing age range and are having or procuring children, potential exposure by pregnant women to media stories can be high. However, in the main, these women did not feel influenced by what celebrities were doing. A moderate positive relationship shown in the results between weight worry and celebrity interest does also point to the probability that those who worry about their weight are being exposed to the media stories of celebrities quickly regaining body shape after childbirth. 


\subsubsection{Transition Success}

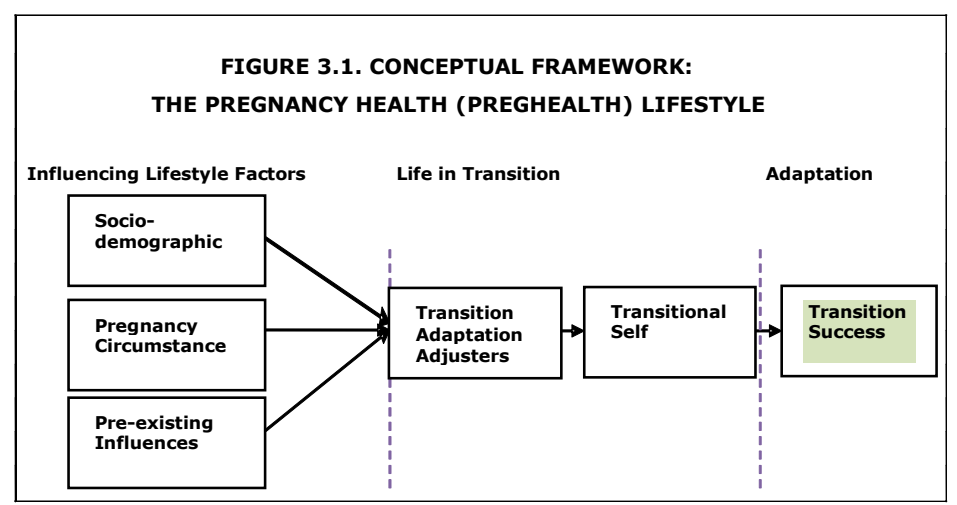

In regard to wellness, on the whole, women did not feel better pregnant than when not pregnant. There was no difference found across trimesters, but those women who had an original pregnancy intention felt better than those who did not. There is some discussion about the 'bloom' or 'glow' of pregnancy' where women feel better than they usually do (Selwyn, 2008) however, this was not shown in this study. For this item, there was a higher mean in those with a pregnancy intention than for those with an unwanted pregnancy. Tiredness and sleep disturbance were certainly features of the women's pregnancies. As adequate sleep and rest are well accepted features of health (Kirsch \& Avidan, 2005; Tunc, Karadag, Dogulu, \& Inan, 2007; Hensley, 2009) a disruption to these patterns will decrease the ability to maximise a health promoting lifestyle in pregnancy.

A sense of attachment to the baby, measured by maternal-fetal attachment, was felt strongly in this group of women. It also aligned positively with other indicators of positive adjustment in this study, as has found by several other previous authors (such as Müller, 1994; Priel \& Besser, 2001). Apart from the 'did not want' category, there was no significant difference between attachment and original intendedness of the pregnancy. Most women were also very much looking forward to the future, although feelings of being 'happy to leave childless days behind' are not quite as positive. Overall, transition as measured in this study, was demonstrated by most to be a successful event, in which positive adaptation to a changing environment and life course was progressing well. 


\subsubsection{SEGMENTATION}

The previous section of the discussion presented an overview of the health lifestyle of the women in this study as a whole. Segmentation allowed for segmentation of the group on the basis of the health psychographic variables that had been used in this study. Segmentation was undertaken first at a sub-cluster phase and then a final summary 'Gravidographic' phase. The benefit of assessing at the sub-cluster level initially was that specific attitude and behaviour sets could be investigated in more detail to obtain rich insights and detail about a specific domain. This then allowed the most salient variables to be identified and put forward for the final Gravidographic phase. The 'Information Attitudes subcluster' results were presented in Chapter 5 as an example of the procedure. The findings of the sub-segments will not be discussed, as although many of the findings were of interest, the exercise was primarily undertaken for data reduction.

\subsubsection{Formation of segments and segmentation patterns}

This section of the chapter discusses the second research question; 'Do health behaviours of pregnancy cluster and what are the underlying factors by which discreet behavioural segments can be identified?' The nature of the data base for this study provided a variety of possibilities for segmentation and it has been demonstrated that segmentation can be undertaken at both an overview level (Gravidographic clusters) and at a more micro level (pre-sub-clusters and subclusters).

The spontaneous breaking of segments into two, three or four groups during Gravidographic clustering and sub-clustering was not dissimilar to the findings of Kolodinsky and Reynolds (2009) in regard to the segments they found. These authors reported a high performing group, low performing in comparison and then one to two groups made up of a mix of both characteristics. The findings are also consistent with Hageol et al. (2002) with their findings of three groups: the most health promoting behaviours had the most privileged circumstances (education, work status), those with the inactive or health compromising patterns had the poorest socio-cultural circumstances and the ambivalent group, made up of some heath-comprising behaviours but mixed with health-enhancing behaviours, circumstances between the two. 
For some attitudes and behaviours, there was an amount of heterogeneity between all the women in the survey (for instance, the wearing of car safety belts). Many of the scales and items were scored highly (or low, where applicable). It may be that women sampled, being well into the second trimester and with increasing attachment and felt responsibility toward the baby (in regard to nurturance and protection), had a more homogeneous dictate for attitudes, decisions and actions that nurture and protect. This is as opposed to a health context where only self was affected, or that might be found in a broader population being studied for health prevention/maintenance behaviour.

It seems that pre-existing influences and patterns are factors that act on choices made in the pregnancy lifestyle. When examining both the Gravidographic and sub-clusters, there was often a consistency seen within a cluster across behaviours with them either being health promoting or alternatively, non-health promoting. There were some exceptions (for instance, the segment that continues to drink alcohol but overall generally takes positive actions). Pregnancy circumstance provides a defining point for many of the relationships tested (although by no means all) and seems to be a major determinant in how the segments spontaneously formed in the 'Gravidographic' segmentation, with those with a pregnancy wish demonstrating the greatest likelihood of health promoting behaviour. In addition, older age and higher socio-economic status align themselves to better performing groups (except in the case of alcohol intake).

Those likely to show the least positive behaviour were younger, did not 'intend' to become pregnant, and if Māori were more likely to be in these groupings. However, as Māori women do tend to have their first baby younger than Caucasian women (Ministry of Health, 2004), it is not possible to say whether it is young age, ethnicity or a mixture of the two that is the main influential agent. A better explanation of why young Māori women are represented in the poorer behaving cluster is needed before determining causality, as it is a complex area. Removing of the 'pregnancy intention' variable from the Gravidographic clustering might well have altered the way segments formed. However, this was not done, as there was strong conceptual support that pregnancy intention is a manifest health lifestyle decision in contemporary society, even if a great number are 'unplanned'. Therefore, intendedness was felt to be salient as a manifest health behaviour and it was valid to go forward into the main clustering. 
Most women are motivated to try to improve health practices for their own well being and more significantly, that of their baby, whether the pregnancy was 'planned' or mistimed (and to a degree, unwanted). This occurred across all segments and there was no backward momentum observed in any cluster. Difference was seen as a measure of degree forward, not opposing direction. Attitude differences are seen in segments but are less distinct points of difference than actual behaviours.

In many ways, the Gravidographic segmentation could be seen as similar to the findings of Kolodinsky and Reynolds (2009), whose study had three segments: one whose health was good, one who performed poorly when compared to the first and a segment 'in the middle'. Firstly, in the Gravidographic segments, the leading one is 'Planners and strong pursuers of health' (C1), those who have high health attitudes and behaviours and who plan their health. Being the cluster with the highest percentage of 'wanted earlier' it is possible that this has been a motivator for organising for and pursuing health promoting behaviour prior. The third segment, 'Lesser attention to health' (C3), practised lesser health promoting behaviour, both before and during pregnancy on many dimensions. Moreover, this segment was made up mainly of mistimed or unwanted pregnancies and is linked to a lower level of resourcing for health. The second and fourth segments, 'Mixed responders' (C2) and 'Slow starters but catch up' (C4) respectively, together form a 'middle ground', where attitudes and behaviour indicators are similar in some areas to the first segment, but lower in others. The second segment can be distinguished as the one with the highest number of women who continue to consume alcohol during the pregnancy, with measures for some information and motivation variables similar to the third cluster. However, the second and fourth segment can be distinguished from each other on profiling variables, such as socio-economic ('Mixed responders' has a higher-sociodemographic level) and pregnancy planning ('Mixed responders' has a higher level of planned or wanted earlier, compared to 'Slow starters but catch up'). 


\subsubsection{THE 'CAUSAL RECIPE' OF PREGNANCY LIFESTYLES}

This chapter sub-section addresses two of the research questions: 'Does information as a transition adjuster play a primary role in the way a pregnancy health lifestyle is enacted?' and 'Are there 'causal recipes' that lead to certain health behaviour styles?

In this study causality cannot be inferred, but the research has pointed to relationships among factors than seem to come together as a causal recipe (Allen, 2008). A poorer health performance in pregnancy has been shown to be associated with an ingredient mix of younger age, unplanned pregnancies and a risk of poorer resourcing for effective health choices (education, income and employment). Conversely, higher levels of health performance are backed by a causal recipe of pregnancy planning and good levels of resourcing to maintain or make appropriate health choices. Indeed, these findings are supported by prior research into the health of individuals and communities by other authors (Altfeld, Handler, Burton, \& Berman, 1998; Green, 2002). Level of health performance within Gravidographic lifestyles is also positively associated with health behaviour prior to pregnancy, pre-existing health attitudes, time orientation and health information seeking and use (again factors supported by previous studies) and giving further weight to Devine and Lepisto's (2005) claim that health trajectories are stable over the life course, whereby pre-existing patterns of health attitudes and behaviours can be seen that hold through the dimensions of the pregnancy conceptual framework.

The existing theories and frameworks that informed the conceptual model for this study, such as The Consumer Decision Making Process Model (Schiffman et al., 2005 have been useful in for the initial conceptualisation of the pregnancy health lifestyle. Information is available through multiple sources and most women are interested and receptive. There is little difference between all of the segments in their reported change to attitudes and behaviour as a response to information. However, the pattern of findings has highlighted the need to better understand health choices and their origins. It cannot be expected that rational choice is the predominant mode of decision making, particularly where socio-historical forces are in action and provide an easier decision route for women (Allen, 2008). For instance, the concept of 'just knowing' is well worth further investigation as to what it really means for choices made in this life transition. 
The behaviours measured in the 'Transitional Health Self' could in part be explained by rational decision making based on information acquisition and response. The higher the level of health promoting behaviour, the higher information is valued and sought. However, rational decision making does not fully explain the situation. Even when socio-economic factors and personality traits are taken into account, a range of in-cluster and between-cluster trajectories and patterning can be seen. This occurrence takes place also in a healthcare system where the same level of information, access and quality of pregnancy health care is arguably available for all. Therefore, the reasons behind choices made regarding life course decisions (for example, when to enter motherhood) do not seem to be rational to the observer, other than 'it just feels right' to the observed. The decision over when and in what situation to become pregnant may well be a consumer behaviour into which the 'less rational' model fits, such as Allen's (2008) Fits-Like-a-Glove (FLAG) model of consumer choice. This model could well be beneficial in future research in this area.

The Fits-Like-a-Glove (FLAG) model of consumer choice (Allen, 2008; Woodside, Nielson, Walters, \& Muller, 2008), which "elaborates on practice theory and integrates elements of social context in a model of choice" (Woodside et al., 2008 , p. 17), seems more useful than the rational information-based consumer decision model in explaining how women make and enact health choices. The FLAG framework is put forward here as an effective way to illuminate why the sub-segments and Gravidographic segments formed as they did. Consumers make decisions on what feels right for them or seems the natural thing to do in a situation. In that way, the 'object of choice' is almost pre-determined. The FLAG choice framework (Allen, 2008) recognizes that choice is not able to be reduced to a set of rational or constructive choice theory. Furthermore Allen (2008) states that "consumer choices are experienced in the flow of a person's life and social relationships with others, which in turn are nested within social collectivities and historical contexts" (p. 528). By emphasizing the socio-historical context, "FLAG choice may shed light on the choices people make that perpetuate gross inequalities, reduced life chances and misery" (Allen, 2008, p. 529).

\subsubsection{SUCCESSFUL TRANSITION}

The measures of success in pregnancy were drawn from the literature as to what makes a 'successful' transition: wellbeing, attachment and orientation to the future. The extent to which one lifestyle group showed more 'transition success' over another is discussed here in light of the study findings. Each health lifestyle 
segment had high Likert scores on all measures tested in this sub-domain. However, the first ('Planners and strong pursuers of health') and fourth ('Slow starters but catch up') segments consistently demonstrated higher sample means than the two other segments and were significantly different ( $95 \%$ confidence) on several measures. It can therefore be said, that on the measures of transition success used in this study, there was a pattern of consistency between level of health performance and transition that was positively related.

As a caution, the measures used here could be described as 'soft' and do not necessarily provide a complete description of success. As the study is a snapshot of a respondent's life whilst still pregnant, the period of transit is still active. In addition, a significant success measure would be birth outcomes and retention of good development and behavioural outcomes for mother and baby into the post partum period with no negative sequalae. These could not be measured within the design of the study and variables selected for measurement are primarily measuring the psycho-social domain of transition only, thereby posing a degree of limitation. However, although these are slightly unconventional, they provide a valuable insight into women's self-experience of transition state and 'readiness' expressed to move onward. Therefore, Research Question 5: Is 'success' in pregnancy transition associated with any particular lifestyle group over another? has been answered.

\subsubsection{PREGNANCY HEALTH CHANGES AND OTHER HEALTH TRANSITIONS}

There are two research questions addressed in this sub-section of this discussion chapter. First, 'what observations can be made in regards to pregnancy as a transitional health life stage and whether changes made are likely to be permanent or for a time only?' (Research Question 6). Second, 'are the health behaviour changes made during pregnancy transition reflective of all life stage transitions, or are there factors that are unique?' (Research Question 7).

Sacrifices made to adjust to a new health state are not uncommon in health. For example, a newly diagnosed diabetic exchanges dietary sugar to achieve better blood glucose levels. However, adjustments made based on the need to protect and nurture the baby may well be a unique factor in terms of motivation. The results of lifestyle changes are driven more by short term focus (the gestation period) rather than long term (for example, a young adult giving up smoking to reduce lung cancer risk in mid to later life). The conceptual framework is probably also a better fit for health transitions as opposed to transitions through 
the lifecycle where other lifestyle factors, eg., retirement, where lifestyle reevaluation and change may be indicated and acted on, but not solely on the basis of changing health) are an equally powerful factor. Furthermore, the model is individual centric rather than household based. If the model was to be proposed as generalisable for household health in transition into Full Nest 1, a future study should include dyadic decisions and actions that couples make together. A lifestyle study grounded in marketing theory of couples in transition into first time fatherhood would be appropriate also, given the rising level of interest in the health of males during this phase.

The scope of the study does not allow demonstration of whether changes made to improve health during pregnancy remain permanent after giving birth and consequently, no definite comments can be made. However, it seems that care and concern for the baby is a key underlying driver of improved health efforts in pregnancy. Given the extent of the changes made that can be seen as sacrificial (food, tobacco, alcohol, energy), in exchange for 'not feeling better than they normally do', it would be debatable as to whether these would be retained once the reason is gone. Authors such as Devine et al. (2000) have shown a tendency to resume normal patterns of health behaviour the post partum period after pregnancy. In particular, when the period of adjustment to new motherhood is known to be a demanding and stressful time, care of self can become a lesser focus.

Therefore, all that can be said is that whilst it can be concluded women do change to adapt to pregnancy to increase the likelihood of positive outcomes, it may possibly be more a trajectory, within a modest range, than a turning point or transition that leads to a marked and/or permanent change in health lifestyle.

\subsubsection{TARGETING PREGNANT AUDIENCES FOR HEALTH COMMUNICATION}

This sub-section discusses the question, 'assuming segments exist, how can each be reached for effective health management, social marketing and health promotion messages that would lead to the adoption of those health behaviours that lead to best mother/baby outcomes?' (Research Question 8). Women appear receptive to health messages during this period. Those with a pregnancy wish are better positioned to hear and respond to messages prior to and at conception (that can be crucial to early foetal development). Women use a range of media to access information. The LMC is a key source and is most highly regarded. 
Likewise, the social and support environment women live within produces an information and advice resource system that pregnant women regard as useful.

There are clearly some health behaviour issues raised by the study which health promotion and communication could address. For instance, a high rate of smoking prior to pregnancy was found, despite this cohort of women having been exposed to anti-smoking messages since infancy. If social marketing messages are designed, persuasion based messages that focus on foetal development and the baby would be the most effective drive in changing attitudes and possibly direct behaviour for all segments. However, it could be argued using strong fear appeals that suggest harm to the baby as a consequence of their behaviour may not be fair, given the high level of motivation to comply for the 'sake of the baby' that already exists.

In regard to specific targeting toward segments, 'Planners and strong pursuers of health' (C1) demonstrate the highest level of health promoting pregnancy behaviours of the segments and are very likely to have planned their pregnancy and possibly have spent time in pre-pregnancy preparation (especially if they required fertility treatment). This segment is comprised of women most likely to be in the higher socio-economic grouping for the study. They have a high desire for and tolerance of information. They can be accessed through main stream media, have good access to the Internet and some have a good readership level of business media.

'Planners and strong pursuers of health' (C1) and 'Lesser attention to health' (C3) are distinctly different (as measured by $95 \%$ confidence for means) in circumstances of their pregnancy, age and socio-economic resources. These two segments need to be regarded as different, not just in attitudes and behaviours but probably in the way messages are received and processed and the how they can be reached.

'Mixed responders' (C2) and 'Slow starters but catch up' (C4) have much in common in regard to attitudes and many behaviours, but vary in other respects. For instance, 'Slow starters but catch up' have a different demographic profile. 'Mixed responders' (C2) and 'Slow starters but catch up' (C4) could benefit from some similar messages, such as pre-pregnancy folic intake, but their different demographic profiles would need to be considered. However, C2 displays a demographic profile to $\mathrm{C} 1$. Therefore consideration of different placement or 
design of messages in targeted media would possibly lead to a more effective communications campaign. In addition, 'Mixed responders' (C2) would be the segment to target in regard to alcohol avoidance. 'Mixed responders' (C2) may have some resistance, however, to information and recommendation in the mass media. In regard to alcohol avoidance communication campaigns, more needs to be known about why this cluster continues to consume alcohol in relatively high numbers. Whether it is a lack of motivation to cease, a lack of information, based on their socio-economic profile or knowledge with a resistance to comply, research findings about alcohol in pregnancy are inconsistent and cannot be determined in this study.

'Lesser attention to health' (C3) is the segment most worthwhile targeting for pregnancy planning and positive health behaviour changes for pregnancy (in particular, smoking cessation). They are least resourced for health (in terms of income and education) and have lesser access to the Internet than other segments. The younger age of many of the members must be an important consideration in how to reach these women and in making messages resonate so that communication goals can be achieved.

It is clear from the study that women value partner involvement highly in events to do with their pregnancy healthcare. Women seem to desire this not just for themselves, but use it as a conduit to provide an environment for their partners to attach to the pregnancy and baby. Therefore, the role and inclusion of fathersto-be in social marketing messages could increase receptivity if women feel that fathers are recognised as co-producers of a healthy lifestyle that in turn will increase their baby's wellbeing.

Whether respondents are receptive to messages extending outside of or beyond the pregnancy period cannot be determined. However, the findings of this study cannot advocate pregnancy as a time to reach women for other health messages, outside of pregnancy. The focus is on the transitional stage and although plans may have been put in place for the post-partum, it would be better to attempt to reach women at that actual stage. The pregnancy period is information and guideline rich, to the extent of possible information overload and needs to be a consideration before any further targeting with postnatal health messages. 


\subsubsection{COMMENTS ABOUT THE CONCEPTUAL FRAMEWORK AND INFORMING THEORIES USED.}

The extended Consumer Decision Making Process Model (CDMP) (Schiffman et al., 2005) was used as the principle contribution to the conceptualisation of the study's framework, and to a large degree was a valuable informing framework. It would seem that information is a key adjuster in pregnancy transition, but a decision making model with information acquisition followed by alternative evaluation based on rational consumer choice is not the full answer. An alternative route, based more on the 'Fits-Like-a-Glove' (FLAG) of Allen (2008) has been discussed and could add value to the framework. Information retrieval and application of knowledge and beliefs (particularly those generated from the socio-historic dimension of the FLAG model of consumer choice may well give a better insight into transitional lifestyle decisions than rational decision making. This is because a framework such as FLAG, takes into account the person's world and decision making reference points. As a result, a woman's 'world' and existing 'habitus' might be emphasised more strongly, as they might well be more influential in leading the woman to the 'best' decision, in regard to her wellbeing and health lifestyle, than information acquisition and rational evaluation of choices.

The Precaution Adoption Process Model (PAPM) (Weinstein \& Sandman, 1992) also served as an informing model to the conceptual framework for the study and has proven to be useful and valid. This health behaviour change model appears well suited to the context of pregnancy preceding an anticipated first birth, as the stages of engagement with potential threats amongst the respondent group are indeed various. It has been seen that those who have been planning pregnancy for sometime are often at a different stage of thought and preparation in regard to behaviours that enhance wellbeing and reduce risk than those for whom the pregnancy was not an expected occurrence.

\subsubsection{SUMMARY/MAIN POINTS OF DISCUSSION}

This study has provided a comprehensive snapshot of women moving toward their first live birth. The respondent group can be described as one where most women have a high degree of efficacy, mastery and health involvement that for most underpin decisions about their pregnancy health. Significant efforts are made to improve health during pregnancy and are most keenly motivated by the wellbeing and safety of the baby; therefore, situational involvement is high. Women are prepared to 'give up' risky health practices as well as consumption 
that is normally regarded as perfectly acceptable practice when non-pregnant (alcohol, delicatessen food etc.). Information is an important driver of the changes made in pregnancy; however, there are other factors that are influential such as pre-pregnancy health and circumstance of the pregnancy. Many sources of information and 'ways of knowing' are used to navigate their way through the pregnancy course. The relationship with their care provider is a key source of information and support, as well as the people around them with whom they spend time. There is a high need and expectation for emotional and practical support from significant others, which appears to be met for most women.

Segments demonstrate distinct collectivities of attitudes and behaviours. The Gravidographic segmentation is defined by four segments. The segment 'Planners and Pursuers of Health' (C1) exhibits a health promoting lifestyle with activities that are consistently positive. There are two segments that demonstrate health promoting lifestyles in many areas, 'Mixed Responders' (C2) and 'Slow Starters but Catch Up' (C3), but are lower in application of some lifestyle components than the 'Planners and Pursuers of Health' segment. A smaller segment 'Lesser Attention to Health' (C4) exists that appears not nearly as well resourced in all respects to practice optimal health promotion. Higher pregnancy intention (applied in this study as a health lifestyle decision women make) was more dominant in the health promoting behaviour segmentation than for lower. Information use and rational decision making appears to be an influential factor, but it is put forward that the FLAG model of decision making may be a better fit than the more conventional models of consumer choice used in marketing to explain the decision situation of this context, which is embedded in a complex network of interwoven pre-existing and circumstantial influences.

The scope of the study does not allow demonstration of whether changes made by a woman during pregnancy to improve health remain permanent after giving birth. Pregnancy is a time or veracious information seeking for most women leading to personal growth and an awareness of health and wellbeing brought about by 'knowledge'. Whilst it can be concluded women do change to adapt to pregnancy and increase the likelihood of positive outcomes, it may possibly be more a trajectory than a turning point or transition that leads to a permanent change in health lifestyle. The strong desire to protect and nurture their baby demonstrated by women, and even if the pregnancy was 'unplanned', indicates that the transitional heath lifestyle of pregnancy assists in leading for most to a significant equipping to prosper as a mother in the postnatal phase. 


\subsection{STUDY LIMITATIONS}

\subsubsection{GENERALISATION AND REPRESENTATION}

The sample did not have a representative number of Pacific respondents. There are two reasons their voice is important. Firstly, Pacific women have been identified as not fully engaging with health services to their advantage in many respects (Ministry of Health, 2004) and the more that can be known about them as health consumers, the greater the possibility of reversing this situation in the future. Secondly, as they are a key user of obstetric services in New Zealand, with proportionally high rates of birth, their low numbers in the study are a potential limitation. Despite initial care and consultation with Pacific midwives in the first phase of data collection and then further targeted sampling in areas where Pacific population is high, the number of Pacific respondents stayed below what was sought. It is not completely known why this low number has been obtained. There is no record of ethnic distribution of questionnaires by providers and this would have certainly improved the calculation of response rates from specific groups of interest. Language problems have been suggested as a causal influence; however, many Pacific women in the childbearing demographic are second generation New Zealanders.

The efforts employed by the researcher to gain participation from these women did not achieve the desired response rate. Suggestions have been made that access through church communities could have led to a higher response. The ability to provide a translation service alongside the questionnaire might have also been of assistance; however the study was not resourced for this.

The overall respondent sample is tending toward the higher educated. This may be a function of the higher educated engaging more in health research because of motivation and interest.

The questionnaire required a moderate standard of literacy and concentration. This may have led to abandonment in some cases, if there were difficulties with concentration or comprehension, given also that the questionnaire was long. Furthermore, as a reasonable level of literacy was required to complete the questionnaire, recent migrants with low levels of written English language skills would not have been able to successfully complete the questionnaire unaided. 
Again, budget constraints did not allow translation of the questionnaire or translators to work alongside women.

Although there was a sufficient Māori women in the study to claim representation with the New Zealand population, the study questionnaire cannot claim to fully canvas Māori childbearing attitudes and practices. There would indeed be additional benefits and insights gained from the study of Māori childbearing health approached from Māori research paradigms and approaches, which this study has not used.

The New Zealand system is unique in terms of maternity health care, because of the independent practice of midwives. This means that the perception and attitudes of this sample of women may well have been influenced by the health service delivery system they were encountering. There may be differences between other countries, where the partnership model, the prescribed model of care in New Zealand, is not practised.

\subsubsection{DISTRIBUTION AND ACCESS}

Conducting health research in New Zealand is a relatively complex activity. A small New Zealand population means that specifically sought health samples and at risk groups can be over researched. Providers can develop a caution to exposing those in their care toward what they perceive as excessive research. In addition, health providers themselves may become fatigued by the demands made on them to participate in or facilitate research. During data collection for this study, the researcher was made aware of several local and two national studies being conducted on the same targeted sample. Therefore, there were some access issues that needed to be worked through.

As a result of the Lead Maternity Carers (LMCS) distributing questionnaires, the researcher did not have contact with the respondent. This led to the potential for loss of accuracy regarding how many questionnaires were actually handed out to women, especially if a number of questionnaire packs had been accepted into a practice where several practitioners worked from. Although efforts were made by the researcher to control the process through follow up phone calls and conversations, the end destination of some questionnaires is not known. 
However, there is no doubt that the researcher having both a marketing and health background was helpful in this research. Firstly, the researcher had knowledge of how to work within some of the confines of 'the system' and secondly, particularly in terms of engagement by LMCs, the researcher was seen as credible to work in the field.

\subsubsection{BOUNDARIES OF STUDY}

As this research provides a snapshot only of a woman's pregnancy and no birth or post-partum outcomes are known, there can be no assumptions made as to whether membership of a particular lifestyle cluster will predispose women to certain birth outcomes. A longitudinal study spanning pregnancy, birth and the infant years would have provided better evidence for this, but the logistics and practical difficulties of such a study would have be extremely large, if not insurmountable.

More information could not be drawn as to whether the changes made during the transitional lifestyle of pregnancy are permanent and what a new normal (Mercer, 2004) means in regard to health intent and practices. A longitudinal study would shed light on whether health in pregnancy is merely a temporary trajectory within lifelong pattern or in fact does serve as a turning point in regard to health attitudes and practices.

The study used a snapshot approach as opposed to a longitudinal design. There is a large existing body of knowledge that supports difference at various stages of the pregnancy. Moreover, the underlying conceptualisation of this study also promotes the transitional lifestyle as one of progression and advancement in areas such as attachment and increased focus on the approaching birth as the pregnancy ages. The results of this study found no significant differences by gestational group on relationships measured. For instance, attachment to the baby showed no significant difference at different gestational points. This can most probably be explained by the fact that the sample was gathered from those in active transition, mostly in the middle part of their pregnancy. The earlier and late stages of the third trimester were not canvassed, because of the recognised dynamic nature of these periods, such as ambivalence in the first trimester and feeling 'fed up' in the last weeks. 
Questionnaires were self-report, so actual responses could not be objectively measured. There was also no clinical assessment or audit of medical or midwifery records, which may also have added more accuracy. However, this would have somewhat taken away from the 'women's voice' in this study, which was important to the study objectives. For instance, when respondents were questioned about risk status, it was their own perception of what particular risk status they had. Access to LMC records would have provided more accurate diagnostic value as to the type and severity of risk women had.

The health pregnancy lifestyle is also clearly not experienced by women in isolation from the family and community to which they belong. In particular, the importance of the woman's partner as a co-producer of lifestyle is acknowledged and although the social network is recognised within the study, the full extent to which a woman makes choices on the basis of another person's input or demands has not been explored in depth.

\subsection{RECOMMENDED FUTURE RESEARCH}

\subsubsection{FURTHER DEVELOPMENT OF THE QUANTITATIVE TOOL}

The questionnaire was comprehensive in evaluating attitudes and practices of the pregnant health lifestyle. As the questionnaire was designed specifically for the study, it was largely untested and therefore exploratory. Some further iteration and refinement is recommended prior to future use. Further work on scales and items used to measure variables in this study could also mean a more streamlined future tool, meaning less work for respondents and decreasing potential respondent fatigue. Removal of less salient variables may then allow for other dimensions of enquiry to be added, such as purchases made to support health, without a further burgeoning of questionnaire length.

\subsubsection{WIDER COUNTRY, CULTURAL AND CONSUMPTION ASSESSMENT}

Measurement in different countries and cultures would be useful to provide insight into whether study findings are stable in different contexts such as culture and healthcare systems. Furthermore, study of multiparous women would provide greater understanding of how women manage health in subsequent pregnancies, where past experience provides knowledge and direct influence over attitudes and behaviours. This would lead to increased robustness of the Gravidographic framework this study contributes as a major output. The concept of Gravidographics could also be expanded to segment pregnant women in terms 
of other consumption decisions and choices: for instance, pregnancy specific products (healthcare, fashion, cosmetics) or baby goods (furniture, feeding intent).

\subsection{THEORETICAL CONTRIBUTION AND MANAGERIAL/PRACTISE RECOMMENDATIONS}

\subsubsection{THEORETICAL}

Two key theoretical contributions have been made: evaluation of pregnancy as a transitional health lifestyle and the conceptualisation of Gravidographics.

\subsubsection{The Transitional Health Lifestyle}

A key objective of this study was to investigate the 'transitional lifestyle' as the lifestyle that occurs in major life transitions and those between major stages of the family lifecycle model. It was proposed that information use was a key driver of adaptation and that this may be generalisable into periods of other life transitions, particularly where health is involved. Whilst information is indeed a key concept in transition, findings do raise questions about how respondents make choices, even despite information, the extent to which information is accommodated and the causal root behind choices made. This study has provided an in-depth descriptive overview of pregnancy health lifestyle, enriched with marketing and health variables, to illuminate the journey women undergo to transit successfully.

\subsubsection{Gravidographics}

This study has proven the possibility of segmentation of pregnancy health lifestyles in regard to collectives of health behaviour attitudes and behaviours through the development of a Gravidographic framework, informed by Moschis (1996) and the 'Gerontographic' segmentation undertaken by him into health situations of the elderly and distinct lifestyles derived from that.

The seminal contribution of Gravidographics adds significant value to knowledge about the contemporary maternity market and has provided an early backbone for a research framework of health psychographic study in the area of pregnancy. The conceptualisation of the transitional pregnancy health lifestyle and subsequent framework is also sufficiently flexible to allow for extension into other areas of pregnancy consumption (choice of healthcare, purchase and acquisition of pregnancy, and baby goods). 


\subsubsection{MANAGERIAL/PRACTICE}

\subsubsection{Service design}

In some geographical areas, there are difficulties obtaining a Lead Maternity Carer. Given that the early weeks of pregnancy are a critical period in fetal development, it would be ideal that the LMC relationship be established in the pre-pregnancy period with those who have a pregnancy wish. This would provide a better environment for information exchange about the early weeks of gestation, rather than women relying on other sources of information until well into the first trimester and first contact with their carer. Pre-pregnancy classes and seminars are currently offered by some maternity providers and the continued development of these should be viewed, at the very least, as a very positive product offering.

Information gained in the study on the preferences and needs of pregnant consumers may be used by providers to configure services to match the expectations and needs of women in targeted groups. Better targeting and positioning of services will ultimately increase market efficiencies by better matching of these parties and also from the standpoint of reduced 'costs' and outcomes. Lifestyle dictates the search for goods and services, so it is very feasible that this is a driver of maternity provider service preference.

Additionally, effective communications that provide appropriate information, signal offerings well and ensure target markets are reached through appropriate choice of media and channels, can be devised. Increased information and effective market signals will enable consumers to become more efficient in their decision making, reduce time and 'costs' and mean that providers can feel more confident that both parties in the exchange are in harmony and share similar expectations.

Maternity providers deliver services in a commercial environment that demands a high level of market orientation and business acumen. The application of a lifestyle marketing segmentation strategy within a health marketing paradigm will promote development in this regard, as well as contributing significantly to the pursuit of service excellence in clinical practice. 


\subsubsection{Information and source credibility of LMCs}

Throughout the study, women made clear the high credibility of midwives and doctors as sources of information and value as support agents. Although other sources of information and ways of knowing were operative and in many cases highly regarded themselves, LMCs need to understand their reach and role as agents for advice, particularly based on the intimate one-to-one relationship they share with women, which other information modes cannot enjoy.

There is evidence that some women find information overload and conflicting information a problem. This is hard to control given the wealth of information sources available, the liberty of others to communicate their opinion and the increasing commercialisation of pregnancy health information. Nevertheless, it needs to be kept in consideration and strategies developed by providers to assist women in identifying reliable sources and how to maximise their processing of it, without becoming overloaded.

\subsubsection{Consumer decision making}

Many attitudes and values that direct habits and lifestyle are learned, experienced and modelled through childhood. Similar to many other research findings, it is the younger, less educated and those with an unwanted pregnancy that appear more at risk for less optimal health decisions within the findings of this study. Continued focus on the development of understanding the type of decision processes that governs pregnant consumers' health choices will enable providers to better determine the care goals and plans they devise in consultation with their clients to maximise possible positive health outcomes.

\subsubsection{Knowledge of pregnancy health in the wider community}

Whilst approximately half of respondents' pregnancies were intentional, an almost equal number of pregnancies were not. This means that many health behaviour modifications would have been made post-conception within mistimed and 'unwanted' pregnancies and also even within 'planned' pregnancies. While many of the health improvements, even if made toward the end of the first trimester, are beneficial, it is not the ideal situation. The concept of women having access to information at the most appropriate times suggests that the distribution of 'management of pregnancy health information' needs to be targeted not just to pregnant women but into the wider community and even pre-pubescent groups, so that awareness exists before pregnancy occurs. For instance, authors such as 
Lu (2007) suggest it would be worthwhile if younger women had a sound understanding of pregnancy nutrition before the likelihood of pregnancy.

The concept of 'just knowing' is worth investigating as to its meaning and whether scatter-gun social marketing and health promotion messages could be worthwhile, as useful information may be received, embodied and stored for retrieval at a later date.

\subsubsection{Social marketing campaigns/health promotion communication}

One of the main findings of this study is the volume of health messages women potentially deal with in pregnancy and the ability the mediascape has to flood the market with messages. Pregnant women could be vulnerable to messages that may be less than accurate because of their high motivation to comply for the sake of their baby. Furthermore, when designing health communication campaigns for pregnant women, fear appeals may not be appropriate, based on the audiences existing level of high emotional involvement and concern and motivation to comply for the sake of the baby. Guttman and Salmon (2004) suggest that the application of commercial marketing to health communication has, to date, escaped scrutiny of ethical issues. The extent to which provision of 'health information' to drive traffic to a brand's website for another marketing purpose, such as purchase of a product, is unethical, presents an interesting debate, particularly within an increasingly consumer-driven e-health environment.

Further study into the optimal timing of messages and the degree to which positive changes are maintained would be beneficial. Likewise, assessment as to whether pregnancy is a time to teach health practice beyond pregnancy (for example, feeding of infants and children to avoid obesity) is well indicated. There is a difference of opinion in terms of whether the most enduring changes in behaviour can be achieved through marketing and whether pregnancy is a time when women are receptive to messages that are not pregnancy specific. This has not been determined in this study.

\subsubsection{Making health choices easier for women}

Perhaps one of the most salient recommendations from these findings is that marketers work with other disciplines to make choice easier for women by focusing on pregnancy products that facilitate healthy choice and enable greater

confidence in pregnant women. Marketers could examine the possibilities of specialist products targeting this market, such as a 'pregnancy tick' similar to the 
'heart tick' campaign for food, or 'pregnancy friendly' cafes, where food offerings and safe food handling practices can be assured or made very visible to pregnant women. This might be a more helpful and ethical contribution from marketing to the pregnancy health lifestyle, as opposed to 'jumping on the bandwagon' of the next social marketing topic that further constrains lifestyle without providing a solution.

\subsubsection{Contemporary maternity markets and technology}

This study highlighted the affinity most women in this cohort have with the use of technology in pregnancy. Although the concept of 'trust' in technology was not explored, there were positive attitudes towards it as an aid in pregnancy. Additionally, it would seem that the women have integrated the concepts of technology and pregnancy as a 'natural' event into somewhat of a hybrid, but one that has meaning for them. This may seem like an oxymoron and potentially in conflict with what providers conceptualise as 'normal' or 'natural' particularly in efforts being made to drive current rates of intervention in pregnancy and birth

down. Health providers may need to find a way to integrate understandings of 'technology' into definitions of 'normal', without feeling that their own personally held ideals of pregnancy and birth are being compromised.

\subsection{CONCLUSION AND FINAL REMARKS}

There is little doubt that this type of research has a place in pregnancy and childbearing research. For some time it has been recognised that lifestyle influences health, and pregnancy provide a research arena where several gaps in the literature exist, particularly in marketing.

The main purpose of this study was to examine the health lifestyle of pregnant women from a psychographic perspective. The findings have provided a comprehensive description of the market and advanced theory by exploring and developing the constructs and variables salient to a pregnancy health lifestyle and relationships between those variables, thus achieving Objective 1 . The contribution of an effective marketing segmentation of the pregnant health market, with the focus of the study being women awaiting their first live birth, has achieved Objective 2 . This study has crossed disciplines to provide rich insights into the health lifestyle of pregnant women. In addition, it has provided insights into the segments. These insights can aid future development of strategies and interventions (such as health communication and service 
positioning) for both marketing and health disciplines. Positioning pregnancy health as a transitional health lifestyle also led to an understanding of the attitudes and behaviours that accompany and drive decisions within a health state that exists for a specific, period of time, as posed by Objective 3 .

Like any research into human behaviour, the field is complex. This research is an initial attempt to scope pregnancy health lifestyle by crossing disciplines, but with particular focus on the inclusion of variables used by marketing, but not necessarily used by other interested disciplines. As this was an exploratory study, future research will refine and sharpen this as a tool for researchers and practitioners.

A key theoretical contribution this study has made is in defining pregnancy health from a psychographic perspective, combining both health and marketing variables and generating a first conceptualisation of Gravidographics. Gravidographics acknowledges that pregnant women can be segmented, according to health lifestyle on a number of dimensions, thus providing a framework for future pregnancy health lifestyle research. Insights into the way women negotiate this life transition and experiences salient to them have resulted in recommendations pertaining to the targeting of women for health services and communication. In conclusion therefore, this study has achieved its aims and comprehensively addressed the research questions.

At several times in the conceptualisation of this study, I have needed to confront the issue of 'whether this is marketing' with others, and even a few times with myself. The thesis rightly ends with a strong defence of this subject matter contributing actively to the betterment of health marketing, social marketing and consumer behaviour theory. One could be critical of some sources of the literature to define and conceive variables, or that investigation and enquiry traverses a path that marketing does not normally take. This has occurred because the marketing literature has not as yet debated many of the concepts and issues critical to the study and in particular, within pregnancy. This is not because pregnant women are inactive consumers or that the market lacks vitality. It is probably because this area has not drawn the attention of marketing researchers to date. Marketing has signalled its interest in health for some years now. If marketing is to be a credible force in health affairs (remembering that the greater percentage of social marketing's efforts directly relate to healthrelated issues (Donovan \& Henley, 2003; Fuhrel-Forbis, Nadorff, \& Snyder, 2009) 
it cannot be poorly informed. It is highly unlikely that marketing will exit health in any near future; to the contrary, it is becoming firmly embedded in the industry. This study has also stretched into the grey spaces between the marketing and health disciplines. This can only be seen as a strength, as it will lead to more synergistic, comprehensive and reliable theoretical understanding with robust application and implementation of marketing strategies. 


\section{REFERENCES}

Aaronson, L.S., Mural, C.M., \& Pfoutz, S.K. (1998). Seeking information: Where do pregnant women go? Health Education and Behaviour, 15(3), 335-45.

Abel, T. (1991). Measuring health lifestyles in a comparative analysis: Theoretical issues and empirical findings. Social Science \& Medicine, 32(8), 899-908.

Adler, A. (1917). The neurotic constitution. New York: Moffat, Yard and Company.

Adler, A. (1927). Individual psychology. Journal of Abnormal and Social Psychology, 22(2), 116-122.

Adler, N.J. (1997). International Dimensions of Organisational Behaviour. Cincinnati, Ohio: South-Western Publishing.

Agazio, J. P. R. N., \& Buckley, K. M. P. R. N. (2009). An Untapped Resource: Using YouTube in Nursing Education. Nurse Educator January/February, 34(1), 23-28.

Ahmed, S.A, \& Jackson, D. N. (1979). Psychographics for social policy decisions: welfare assistance. Journal of Consumer Research, 5(4), 229-39.

Aldoory, L. (2001). Making Health Communications Meaningful for Women: Factors That Influence. Journal of Public Relations Research, 13(20), 163-185.

Allen, D. E. (2002). Toward a Theory of Consumer Choice as Sociohistorically Shaped Practical Experience: The Fits-Like-a-Glove (FLAG) Framework. The Journal of Consumer Research, 28(4), 515-532.

Almeida, J., Molnar, B.E., Kawachi, I., \& Subramanian, S.V. (2009). Ethnicity and nativity status as determinants of perceived social support: Testing the concept of familism. Social Science and Medicine, 68, 1852-8.

Alreck, P., \& Settle, R. (2004). The survey research handbook (3 ${ }^{\text {rd }}$ Ed.). New York: McGraw.

Altfeld, S., Handler, A., Burton, D., \& Berman, L. (1997). Wantedness of pregnancy and prenatal health behaviors. Women \& Health, 26(4), 29-43.

Amy, J., \& Loeber, O. (2007). Pregnancy during adolescence: A major social problem. European Journal of Contraception and Reproductive Health Care, 12(4), 299-302. 
Anderson, W.T., \& Golden, L.L. (1984). Life style and psychographics: a critical view and recommendation. In T.C. Kinnear (Ed.), Advances in Consumer Research, 11, (pp. 405-11).

Ando, A., \& Modigliani, F. (1963). The "life cycle" hypothesis of saving: Aggregate implications and tests. American Economic Association, 53(1), 55-84.

Andreasen, A.R. (2003). The life trajectory of social marketing: Some implications. Marketing Theory, 3, 293-303.

Angus, J., Rukholm, E., St. Onge, R., Michel, I., Nolan, R.P., Lapum, J., \& Evans, S. (2007). Habitus, stress, and the body: The everyday production of health and cardiovascular risk. Qualitative Health Research, 17, 1088-1101.

Antoniou, M., Tzouvali, H., Sifakis, S., Galanakis, E., Georgopoulou, E., Liakou, V., et al. (2004). Incidence of toxoplasmosis in 5532 pregnant women in Crete, Greece: management of 185 cases at risk. European Journal of Obstetrics \& Gynecology and Reproductive Biology, 117(2), 138-143.

Antononucci, T.C., \& Mikus, K. (1998). The power of parenthood: personality and attitudinal changes during the transition to parenthood. In G.Y. Michaels \& W.A Goldberg (Eds.), The Transition to Parenthood: Current Theory and Research (pp.62-84). Cambridge: Cambridge University Press.

Ardell, D.B. (1977). High Level Awareness. Emmaus, PA: Rodale Press.

Armstrong, D., \& Hutti, M. (1998). Pregnancy After Perinatal Loss: The Relationship Between Anxiety and Prenatal Attachment. Journal of Obstetric, Gynecologic, \& Neonatal Nursing, 27(2), 183-189.

Arndt, J. (1978). How broad should the marketing concept be? Journal of Marketing, 43, 101-3.

Aronson, T. (1994). A pragmatic view of thematic analysis. The Qualitative Report, 2(1).

Ashdown-Lambert, J. (2005). A review of low birth weight: predictors, precursors and morbidity outcomes. The Journal of the Royal Society for the Promotion of Health, $125(2), 76-85$.

Ayaz, S., \& Efe, S.Y. (2008). Potentially harmful traditional practices during pregnancy and postpartum. European Journal of Contraception \& Reproductive Health Care, 13(3), 282. 
Azjen, I., \& Fishbein, M. (1980). Understanding attitudes and predicting behaviour. Englewood Cliffs: Prentice-Hall.

Azjen, I. (1985). From Intentions to Actions: hoery of planned behaviour. In J. Kuhl, \& J. Beckmann (Eds.), Action-Control: from cognition to behaviour (pp.11-39). Heidelberg: Springer.

Ajzen, I. (1991). The theory of planned behavior. Organizational Behavior and Human Decision Processes, 50(2), 179-211.

Bagozzi, R. P. (1992). The Self-Regulation of Attitudes, Intentions, and Behavior. Social Psychology Quarterly, 55(2), 178-204.

Balen, F., Naaktgeboren, N., \& Trimbos-Kemper, T. C. M. (1996). In-vitro fertilization: the experience of treatment, pregnancy and delivery. Human Reproduction, 11(1), 95-98.

Bhalla, A. K., Sarala, G., \& Dhaliwal, L. (1992). Pregnancy Following Infertility. Australian and New Zealand Journal of Obstetrics and Gynaecology, 32(3), 249251.

Barba, E., \& Selder, F. (1995). Life transitions theory. Nursing Leadership Forum, 1, 411.

Barker, D, J., Osmond, C. (1986). Infant mortality, childhood nutrition, and ischemic heart disease in England and Wales. Lancet, 1, 1077-1081.

Bearden, W., Netemeyer, R., \& Teel, J. (1989). Measurement of consumer susceptibility to interpersonal influence. Journal of Consumer Research, 15, 473-81.

Belgrave, M. (1991). Medicine and the rise of the health professions in New Zealand, 1860-1939. Healthy Country: Essays on the Social History of Medicine in New Zealand. Wellington: BWB.

Bell, E. And Bryman, Alan: (2003). Business Research Methods. Second edition, Oxford University Press.

Bergart, A. M. (2000). The Experience of Women in Unsuccessful Infertility Treatment -What Do Patients Need When Medical Intervention Fails? Social Work in Health Care, 30(4), $45-69$.

Berry, E.H., Shillington, A.M., Peak, T., \& Hohman, M.M. (2000). Multi-ethnic comparison of risk and protective factors for adolescent pregnancy. Child and Adolescent Social Work Journal, 17(2), 79-96. 
Berry, H.L. (2008). Social capital elite, excluded participators, busy working parents and aging, participating less: types of community participators and their mental health. Social Psychiatry and Psychiatric Epidemiology, 43(7), 527-37.

Berryman, J. (1991). Perspectives on late motherhood. In A. Phoenix, A. Woollett, \& E. Lloyd (Eds.), Motherhood: Meanings, Practices and Ideologies. London: Sage.

Blackwell, R.D., Miniard, P.W., \& Engel, J.F. (2001). Consumer Behavior ( $9^{\text {th }}$ Ed.). Orlando, FL: Harcourt.

Bloch, P. H. (1984). The wellness movement: Imperatives for health care marketers. Journal of Health Care Marketing, 4, 9-16.

Bond, M. L., Jones, M. E., Cason, C., Campbell, P., \& Hall, J. (2002). Acculturation effects on health promoting lifestyle behaviors among hispanic origin pregnant women. Journal of Multicultural Nursing \& Health, 8(2), 61-68.

Bondarianzadeh, D. (2007). Food risk to babies. Nutrition Today, 42(6), 236.

Bondas, T., \& Eriksson, K. (2001). Women's lived experiences of pregnancy: A tapestry of joy and suffering. Qualitative Health Research, 11(6), 824-840.

Borgatta, E.F., Bulcroft, K., Montgomery, R.J.V., \& Bulcroft, R. (1990). Health promotion over the life course. Research on Aging, 12, 373-388.

Bourdieu, P. (1984). Distinction. Cambridge, MA: Harvard University Press.

Bourdieu, P. (1990). The Logic of Practice. Cambridge, UK: Polity.

Bourdieu, P. (1999). Site effects - The Weight of the World: Social Suffering in Contemporary Society. Stanford, CA: Stanford University Press.

Bristor, J., \& Quall, W. (1984). The household life cycle: implications for family decision making. In R. Roberts \& R. Wortzel (Eds.), Marketing to the Changing Household. Cambridge: L.MA Ballinger.

Brown, J.E., Murtaugh, M.A, Jacobs Jr, D.R., \& Margellos, H.C. (2002). Variation in newborn size according to pregnancy weight change by trimester. American Society for Clinical Nutrition, 76(1), 205-09.

Bruwer, J., Li, E., \& Reid, M. (2002). Segmentation of the Australian wine market using a wine-related lifestyle approach. Journal of Wine Research, 13(3), 2217-42. 
Brunso, K., Scholderer, J., \& Grunert, K. G. (2004). Closing the gap between values and behavior - a means-end theory of lifestyle. Journal of Business Research, 57, 665-670.

Bushman, F.A. (1982). Systematic life styles for new product segmentation. Academy of Marketing Science. Journal, 10(4), 377-94.

Butler, S., Williams, M., Paterson, J., \& Tukuitonga, C. (2004). Smoking among mothers of a Pacific Island birth cohort in New Zealand: Associated factors. Journal of the New Zealand Medical Association, 117(1206).

Cagampang, H.H., Barth, R.P., Korpi, M., \& Kirby, D. (1997). Education now and babies later (ENABL): Life history of a campaign to postpone sexual involvement. Family Planning Perspectives, 29(3), 109-14.

Campbell, M.K., Mottola, M.F. (2001). Recreational exercise and occupational activity during pregnancy and birth weight: A case-control study. American Journal of Obstetrics and Gynaecology, 184(3), 403-8.

Carrigan, M., \& Szmigin, I. (2004). Time, uncertainty and the expectancy experience: An interpretive exploration of consumption and impending motherhood. Journal of Marketing Management, 20, 771-798.

Casey, E. (2001). Between geography and philosophy: What does it mean to be in the place-world? Annals of the Association of American Geographers, 91(4), 683-693.

Cassidy, T. (2000). Stress, healthiness and health behaviours: An exploration of the role of life events, daily hassles, cognitive appraisal and the coping process. Counselling Psychology Quarterly, 13(3), 293-311.

Cavana, R.,Delahaye, B and Sekaran, U. (2000). Applied Business Research: Qualitative and Quantitative Methods. Milton, QLD: John Wiley and Sons Inc.

Chaney, D. (1996). Lifestyles. London: Routledge.

Chin, N. P., Monroe, A., \& Fiscella, K. (2000). Social determinants of (un)healthy behaviors. Education for Health, 13(3), 317-328.

Chomitz, V.R., Cheung, L.W.Y., \& Lieberman, E. (1995). The role of lifestyle in preventing low birth weight. The Future of Children, 5(1), 121-38.

Clarke, P. E., \& Gross, H. (2004). Women's behaviour, beliefs and information sources about physical exercise in pregnancy. Midwifery, 20(2), 133-141. 
Clark, M., \& Ogden, J. (1999). The impact of pregnancy on eating behaviour and aspects of weight concern. International Journal of Obesity, 23(1), 18-24.

Clissold, T.L., Hopkins, W.G., \& Seddon R.J. (1991). Lifestyle behaviours during pregnancy. NZ Medical Journal, 104(908), 111-12.

Coakes, S. J., \& Steed, L. (2007) SPSS version 14.0 for windows: Analysis without anguish. Milton, Australia: John Wiley \& Sons.

Cockerham, W.C. (2000). Health lifestyles in Russia. Social Science and Medicine, 51(1), 1313-24.

Cockerham, W.C. (2005). Health lifestyle theory and the convergence of agency and structure. Journal of Health and Social Behaviour, 46(3), 51-67.

Cockerham, W.C. (2007). Health lifestyles and the absence of the Russian middle class. Sociology of Health and Illness, 29(3), 457-73.

Cockerham, W.C., Abel, T., \& Luschen, G. (1993). Max Weber, formal rationality and health lifestyles. The Sociological Quarterly, 34(3), 413-28.

Cockerham, W.C., Rutten, A., \& Abel, T. (1997). Conceptualizing contemporary health lifestyles: Moving beyond Weber. The Sociology Quarterly, 38(2), 321-42.

Condon, J.T., Boyce, P., \& Corkindale, C.J. (2003). The first-time fathers study: a prospective study of the mental health and wellbeing of men during the transition toparenthood. Australian and New Zealand Journal of psychiatry, 38(1), 56-64.

Cooksey, N. R. (1995). Pica and Olfactory Craving of Pregnancy: How Deep Are the Secrets? Birth, 22(3), 129-137.

Cooper, D.R., \& Emory, E.W. (1995). Business Research Methods (5 ${ }^{\text {th }}$ Ed.). Chicago: Richard D. Irwin Inc.

Creswell, J.W. (2003). Research design: Qualitative, quantitative, and mixed approaches. Thousand Oaks, CA: Sage Publication.

Cresswell, J W. (2009). Research design: qualitative, quantitative, and mixed. Thousand Oaks, CA: Sage Publication.

Cresswell, J., Fetters, M., \& Ivankova, N. (2004). Designing a Mixed Methods Study in Primary Care. Annals of Family Medicine, 2, 7 - 12. 
Cresswell, J.W., Fetters, M.D., \& Ivankova N.V. (2004) Designing a mixed methods study in primary care. Annals of Family Medicine, 2, 7-12.

Cronin, A., Alexander, V.D., Fielding, J., Moran-Ellis, et al. (2008). The Analytic Integration of Qualitative Data Sources. In Handbook of Social Research Methods, 572-84.

Crossley, N. (2001). The phenomenological habitus and its construction. Theory and Society, 30, 81-120.

Cunningham, F.G., MacDonald, P.C., Gant, N.F., Leveno, K.J., Gilstrap, L.C., III, Hankins, G.D.V., et al. (1997). Williams obstetrics. Stamford, CT: Appleton \& Lange.

D'Angelo, D. V., Gilbert, B. C., Rochat, R. W., Santelli, J. S., \& Herold, J. M. (2004). Differences between mistimed and unwanted pregnancies among women who have live births. Perspectives on Sexual and Reproductive Health, 36(5), 192-107.

David, R., \& Collins, J. (1997). Differing birth weight among infants of U.S.-born blacks, African-born blacks, and U.S.-born whites. The New England Journal of Medicine, 337(17), 1209. Retrieved September 26, 2010, from Research Library.

De Boo, H.A., \& Harding, J.E. (2006). The developmental origins of adult disease (Barker) hypothesis. Australian \& New Zealand Journal of Obstetrics \& Gynaecology, 46(1), 4-14.

De Walle, H.E.K., \& de Jong-van de Berg, L.T.W. (2007). Ten years after the Dutch public health campaign on folic acid: The continuing challenge. European Journal of Clinical Pharmacology, 64, 539-43.

Dean, T., Venter, C., Pereira, B., Grundy, J.T., Clayton, C.B., \& Higgins, B. (2007). Government advice on peanut avoidance during pregnancy, is it followed correctly and what is the impact on sensitization? Journal of Human Nutrition and Dietetics, 20, 95-9.

Dejin-Karlson, E., \& Ostergren, P. (2003). Psychosocial factors, lifestyle, and fetal growth: the added value of both pre- and post-natal assessments. European Journal of Public Health, 13(3), 210-7.

Delgado, A. (2008). Listeriosis in pregnancy. Journal of Midwifery and Women's Health, 53(3), 255-9.

Demby, E. (1974). Psychographics and from where it came. In W.D. Wells (Ed.), Life Style and Psychographics (pp.9-30). Chicago, IL: American Marketing Association. 
DeVellis, R.F. (2003). Scale Development: Theory and Applications. Thousand Oaks, CA: Sage Publications.

Devine, C.M. (2005). A life course perspective: understanding food choices in time, social location, and history. Journal of Nutrition Education and Behaviour, 37(3), 121-28.

Devine, C., Connors, M., Bisogni, C., \& Sobal, J. (1998). Life course influences on fruit and vegetable trajectories. Journal of Nutrition Education, 30, 361-370.

Devine, C.M., Bove, C.F., \& Olson, C.M. (2000). Continuity and change in women's weight orientations and lifestyle practices through pregnancy and the postpartum period: the influence of life course trajectories and transitional events. Social Science and Medicine, 50(4), 567-82.

Deutsch, F., Ruble, D., Fleming, A., Brooks-Gunn, J., \& Stangor, C. (1988). Informationseeking and maternal self-definition during the transition to motherhood. Journal of Personality and Social Psychology, 55, 420-31.

Dichter, E. (1986). Whose lifestyle is it anyway? Psychology and Marketing, 3, 151-163.

DiPietro, J.A., Millet, S., Costigan, K.A., Gurewitsch, E., \& Caulfield, L.E. (2003). Psychosocial influences on weight gain attitudes and behaviors during pregnancy. Journal of American Dietetic Association, 103(10), 1314-1319.

Divine, R.L., \& Lepisto, L. (2005). Analysis of the healthy lifestyle consumer. Journal of Consumer Marketing, 22(5), 275-283.

Dixon-Woods, M., Bonas S., Booth A., Jones D.R., et al. (2006). How can systematic reviews incorporate qualitative research? A critical perspective. Qualitative Research, 6, 27-44.

Dobrzykowski, T.M., \& Noerager Stern, P. (2003). Out of sync: a generation of first-time mothers over 30. Health Care for Women International, 24(3), 242-53.

Dolinksy, A.L., \& Stinerock, R. (1998). Cultural affiliation and the importance of health care attributes. Marketers can develop segmentation strategies for targeted patient groups. Journal of Marketing and Health Services, 18(1), 28-37.

Donley, J. (1986). Save the Midwife. Auckland: New Women's Press.

Donovan, R. (1992). Using Media for Road Safety Public Education Campaigns. Report to the Traffic Board of Western Australia. 
Donovan, R., \& Henley, N. (2003) Social Marketing: Principles and practices Melbourne: IP Communications Pty Ltd.

Durie, M. H. (1989). The Treaty of Waitangi and health care. New Zealand Medical Journal, 102(869), 283-285.

Drake, M.L., Verhulst, D., Fawcett, J., \& Barger, D.F. (1998). Spouses' Body Image Changes During and After Pregnancy: A Replication in Canada. Journal of Nursing Scholarship, 20(2), 88-92.

DuBois, P. (2000). MySQL. Indianapolis: New Riders.

Duffy, M. E., Rossow, R., \& Hernandez, M. (1996). Correlates of health-promotion activities in employed Mexican American women. Nursing Research, 45(1), 18-24.

Dumas, A., \& Laberge, S. (2005). Social class and ageing bodies: Understanding physical activity in later life. Social Theory \& Health, 3, 183-205.

Dunn, C. L., Pirie, P. L., \& Hellerstedt, W. L. (2003). The advice-giving role of female friends and relatives during pregnancy. Health Education Research, 18(3), 352362

Dunlop, A. L., Gardiner, P. M., Shellhaas, C. S., Menard, M. K., \& McDiarmid, M. A. (2008). The clinical content of preconception care: the use of medications and supplements among women of reproductive age. American Journal of Obstetrics and Gynecology, 199(6, Supplement 2), S367-S372.

Dutta, M. J., \& Youn, S. (1999). Profiling Healthy Eating Consumers: A Psychographic Approach to Social Marketing. Social Marketing Quarterly, 5(4), 5-21.

Dutta-Bergman, M. J. (2003). Developing a profile of consumer intention to seek out health information beyond the doctor. Health Marketing Quarterly, 21(1/2), 91112.

Dutta-Bergman, M. J. (2004). Primary Sources of Health Information: Comparisons in the domain of health attitudes, health cognitions, and health behaviours. Health Communication, 16(3), 273-288..

Dutta- Bergman, M. J. (2004). The readership of health magazines: The role of health orientation. Health Marketing Quarterly, 22(2), 27-49.

Dutta- Bergman, M. J. (2004). A descriptive narrative of healthy eating. Health Marketing Quarterly, 20(3), 81-101. 
Dutta-Bergman, M. J. (2006). A formative approach to strategic message targeting through soap operas: Using selective processing theories. Health Communication, 19(1), 11-18.

Duvall, E. (1955). Family Development. Philadelphia: Lippincott.

Earle, S. (2003). "Bumps and Boobs": Fatness and women's experiences of pregnancy. Women's Studies International Forum, 26(3), 245-252.

Elder, G. (1987). Families and lives: Some developments in life-course studies. Journal of Family History, 12, 179-199.

Elliot, A.J. (1997). Integrating the 'classic' and 'contemporary' approaches to achievement motivation: A hierarchical model of approach and avoidance achievement motivation. In M.L Machs \& P.R Pintrich (Eds.), Advances in Motivation Achievement, 10, (pp. 143-79). Greenwich, CT: JAI Press.

Elliot, A. J. (2006). The hierarchical model of approach-avoidance motivation. Motivation and Emotion, 30, 111-116.

Ellison, C. G., \& Levin, J. S. (1998). The religion-health connection: Evidence, theory, and future directions. Health Education and Behavior, 25, 700-720.

Ellison, C.G., Boardman, J.D., Williams, D.R. \& Jackson, J.S. (2001). Religious involvement, stress, and mental health: Findings from the 1995 Detroit Area Study. Social Forces, 80(1), 215-249.

Engelhardt, H., \& Prskawetz, A. (2004). On the Changing Correlation between Fertility and Female Employment over Space and Time. European Journal of Population/ Revue EuropÃ@enne de DÃ@mographie, 20(1), 35-62.

Erdelez, S. (1999). Information encountering: It's more than just bumping into information. Bulletin of the American Society for Information Science, 25(3), 25-9.

Evenson, K.R., Savitz, D.A., \& Huston, S.L. (2004). Leisure-time physical activity among pregnant women in the US. Paediatric and Perinatal Epidemiology, 18(6), 400-7.

Feldman, P., Dunkel-Schetter, C., Sandman, C. A., \& Wadhwa, P. (2000). Maternal social support predicts birth weight and fetal growth in human pregnancy. Psychosomatic Medicine, 62, 715-725.

Fetto, J. (May, 2003). The baby business. American Demographics, p.4. 
Flanagin, A., \& Metzger, M. (2001). Internet use in the contemporary media environment. Human Communication Research, 27(1), 153-181.

Flanders, J. (2003). The Victorian House. Hammersmith: Harper Collins.

Florian, V., \& Mikulincer, M. (1995). Effects of adult attachment style on the perception and search for social support. Journal of Psychology, 129(6), 665.

Field, A, (2009). Discovering statistics using SPSS ( $3^{\text {rd }}$ ed). London: Sage.

Fisher, R. (1922). On the interpretation of chi-square from contingency tables, and the calculation of P. Journal of the Royal Statistical Society, 85, 87-94.

Fougere, G. (1994). Health (in) Spoonley, P \& S. New Zealand Society (2 ${ }^{\text {nd }}$ Ed.). Palmerton North, New Zealand: Dunsmore.

Fowles, E. R., \& Feucht, J. (2004). Testing the barriers for healthy eating scale. Western Journal of Nursing Research, 26(4), 429-443.

Freeman, L.M, Timperley, H., \& Adair, V. (2004). Partnerships in midwifery care in New Zealand. Midwifery, 20(1), 2-14.

Friese, C., Becker., G., \& Nachtigall, R.D. (2008). Older motherhood and the changing life course in the era of assisted reproductive technologies. Journal of Aging Studies, 22(1), 65-76..

Gaffney, L., \& Smith, C. A. (Writer) (2004). Use of complementary therapies in pregnancy: The perceptions of obstetricians and midwives in South Australia: Blackwell Publishing, Ltd.

Garber, M., \& Seligman P. (Eds.) (1980). Human helplessness: Theory and applications. New York: Academic Press, 1980

Genevie, L., \& Margolies, E. (1987). The Motherhood Report: How Women Feel about Being Mothers. New York: Macmillan Publishing Company.

Gennaro, S., \& Fehder, W. (2000). Health behaviors in postpartum women. Family and Community Health, 22, 16-26.

Geronimus, A. T. (1996). Black/white differences in the relationship of maternal age to birthweight: A population-based test of the weathering hypothesis. Social Science \& Medicine, 42(4), 589-597.

Gilly, M.C., \& Ennis, B.M (1982). Recycling the family life cycle: a proposal for redefinition. In A. Mitchell \& A. Ar-bor (Eds.), Consumer Research, 9, 271-6. 
Gluckman, P.D., Hanson, M.A. (2004). Developmental origins of disease paradigm: A mechanistic and evolutionary perspective. Pediatric Research, 56, 311-7.

Godin, G., Valois, P., Lepage, L., \& Desharnais, R. (Writer) (1992). Predictors of smoking behaviour: an application of Ajzen's theory of planned behaviour. Oregon: Blackwell Publishing Ltd.

González, A.M., \& Bello, L. (2002). The construct "lifestyle" in market segmentation. European Journal of Marketing, 36(1/2), 51-85.

Goss, J. (1995). "We Know Who You Are and We Know Where You Live": The Instrumental Rationality of Geodemographic Systems. Economic Geography, 71, (2), 171-198.

Granzin, K. L., Olsen, J. E., \& Painter, J. J. (1998). Marketing to consumer segments using health-promoting lifestyles. Journal of Retailing and Consumer Services, 5(3), 131-141.

Green, J. M., \& Baston, H. A. (2007). Have Women Become More Willing to Accept Obstetric Interventions and Does This Relate to Mode of Birth? Data from a Prospective Study. Birth, 34(1), 6-13.

Green, N.S. (2002). Folic acid supplementation and prevention of birth defects. The Journal of Nutrition, 132(8), 2356-60.

Green-Raleigh, K., Lawrence, J.M., Chen, H., Devine, O., \& Prue. C. (2005). Pregnancy planning status and health behaviours among non-pregnant women in a California managed health care organization. Perspectives on Sexual and Reproductive Health, 37(4), 179-83.

Greene, J.C., Caracelli, V.J., \& Graham, W.F. (1989). Toward a conceptual framework for mixed-method evaluation designs. Educational Evaluation and Policy Analysis, 11, 255-274.

Greene, J.C., \& Caracelli, V.J. (Eds.). (1997) Advances in mixed-method evaluation: The challenges and benefits of integrating diverse paradigms. New directions for Evaluation, 74, 19-32.

Grindstaff, C. F. (1996). The costs of having a first child for women aged 33-38, Canada 1991. Sex Roles, 35(3/4), 137-51. 
Grunert, K.G., Brunso, K., \& Bisp, S. (1993). Food-related lifestyle: development of a cross-culturally valid instrument for market surveillance. Denmark: The Aarhus School of Business.

Guilliland, K. (1999). Midwifery in New Zealand: The New Zealand experience. Paper presented at the Future Birth: The Place to be Born Conference, Australia.

Gunter, B., \& Furnham, A. (1992). Consumer Profiles: An Introduction to Psychographics. London: Routledge.

Gupton, A., Heaman, M., \& Cheung, L. W. (2001). Complicated and uncomplicated pregnancies: women's perception of risk. JOGNN: Journal of Obstetric, Gynecologic \& Neonatal Nursing, 30(2), 192-201.

Gurung, R.A.R., Dunkel-Schetter, C., Collins, N., Rini, C., \& Hobel, C.J. (2005). Psychosocial predictors of prenatal anxiety. Journal of Social and Clinical Psychology, 24(4), 497-519.

Gustafsson, S. (2001). Optimal age at motherhood. Theoretical and empirical considerations on postponement of maternity in Europe. Journal of Population Economics, 14(2), 225-247.

Guttman, N., \& Salmon, C.T. (2004). Guilt, fear, stigma and knowledge gaps: Ethical issues in public health communication interventions. Bioethics, 18(6), 531-52.

Hagoel, L., Ore, L., Neter, E., Silman, Z., \& Rennert, G. (2002). Clustering women's health behaviour. Health Education and Behaviour, 29(2), 170-82.

Haines, L. (2009, January 31). Another unfortunate experiment? New Zealand Listener, $217(3586)$.

Hair, J. F., Jr., Black, W. C., Babin, B. J., Andersen, R. E., \& Tatham, R. L. (2006). Mutilvariate data analysis (6th ed.). Upper Saddle River, NJ: Pearson Prentice Hall.

Hair, J. F., Jr., Black, W. C., Babin, B. J., Andersen, R. E., \& Tatham, R. L. (2010). Mutilvariate data analysis (7th ed.). Upper Saddle River, NJ: Pearson Prentice Hall.

Halliday, S. (2000). Maternity care: Ways to add value. The Services Industries Journal, 20(4), 131-147.

Halliday, S., \& Hogarth-Scott, S. (2000). New Customers to be managed: Pregnant women's views as consumers of healthcare. Journal of Applied Management Studies, 9(1), 55-66. 
Hammersley, M. (2006). Handbook of qualitative research, 3rd edition. British Educational Research Journal, 32(3), 536-541.

Hartweg, D.L., \& Berbiglia, V.A. (1996). Determining the adequacy of a health promotion self-care interview guide with healthy, middle-aged, Mexican American women: a pilot study. Health Care for Women International, 17(1), 57-68.

Harvey, S. M., \& Faber, K. S. (1993). Obstacles to Prenatal Care Following Implementation of a Community-Based Program to Reduce Financial Barriers. Family Planning Perspectives, 25(1), 32-36.

Haslam, C., \& Lawrence, W. (2004). Health-related behaviour and beliefs of pregnant smokers. Health Psychology, 23(5), 486-91.

Haslam, C., Lawrence, W., \& Haefeli, K. (2003). Intention to breastfeed and other important health-related behaviour and beliefs during pregnancy. Family Practice, 20(5), 528-530.

Hastings, G., \& Haywood, A. (1991). Social marketing and communication in health promotion. Health Promotion International, 6, 135-45.

Hatfield, E., \& Sprecher, S. (1986). Mirror Mirror ... The Importance of Books in Everyday Life. Albany: State University of New York Press.

Heaman-Gupton, A., \& Gregory, D. (2004). Factors influencing pregnant women's perceptions of risk. The American Journal of Maternal/Child Nursing, 29(2), 111-6.

Heath, R.P. (1995). Psychographics: Qu'est-ce que c'est? American Demographics, 11, 10-24.

Hellerstedt, W. L., Pirie, P. L., Lando, H. A., Curry, S. J., McBride, C. M., Grothaus, L. C., et al. (1998). Differences in preconceptional and prenatal behaviors in women with intended and unintended pregnancies. Am J Public Health, 88(4), 663-666.

Hendrix, P. (1984). Antecedents and consequences of time use: Proposed measures and preliminary evidence. Advances in Consumer Research, 11, 5-40.

Hensley, J. G. (2009). Leg Cramps and Restless Legs Syndrome During Pregnancy. Journal of Midwifery \& Women's Health, 54(3), 211-218.

Hewlett, S. (2002). Executive women and the myth of having it all. Harvard Business Review, 80(4), 66-73. 
Hirschman, E. C. (1987). Theoretical Perspectives of Time Use: Implications for Consumer Behavior Research. In J.N. Sheth, \& E. Hirschman (Eds.), Research in Consumer Behavior, 2, 55-81.

Hirschman, E., (1991). Babies for sale: Market ethics and the new reproductive technologies. The Journal of Consumer Affairs, 25(2), 358-91.

Hoek, J., Gendall, P., \& Esslemont, D. (1996). Market segmentation: a search for the holy grail. Journal of Marketing Practice, 2(1), 25-34.

Hofberg, K., \& Ward, M.R. (2003). Fear of pregnancy and childbirth. Postgraduate Medical Journal, 79, 505-10.

Höjeberg, P. (1985). Trollmor: Sägner om moderskap [Troll-mother: Legends of motherhood]. Sweden: Gidlunds.

Holt, D. (1997). Poststructuralist lifestyle analysis: conceptualizing the social patterning of consumption in postmodernity. Journal of Consumer Research, 23, 326-50.

Hornik, J. (1990). Time preference, psychographics, and smoking behaviour. Journal of Health Care Marketing, 10(1), 36-46.

Huizink, A. C., Robies de Medina, P. G., Mulder, E. J. H., Visser, G. H. A., \& Buitelaar, J. K. (2002). Coping in Normal Pregnancy. Annals of Behavioral Medicine, 24(2), 132-140.

Hustad, T.P and Pessemier, E.A. (1974). The development and application of psychographic life style and associated activity and attitude measures. In W.D. Wells (Ed.) Life Style and Psychographics, 31-70.

Janz, N.K., \& Becker, M.H. (1984). The health belief model: a decade later. Health Education Quarterly, 11(1), 147.

Johnson, R.B., \& Onwuegbuzie, A.J. (2004). Mixed methods research: A research paradigm whose time has come. Educational Researcher, 33(14), 14-26.

Johnson, S., Burrows, A., \& Williamson, I. (2004). 'Does my bump look big in this?' The meaning of bodily changes for first-time mothers-to-be. Journal of health Psychology, 9(3), 361-374.

Joseph, K.S., Liston, R.M., Dodds, L., Dahlgren, L., \& Allen, A.C. (2007). Socioeconomic status and perinatal outcomes in a setting with universal access to essential health care services. Canadian Medical Association, 177(6), 583-90. 
Kahle, L.R. (1984). Social Values and Social Change: Adaptation to Life in America. New York: Praeger.

Kaiser, L., \& Allen, L. (2008). Position of the American Dietetic Association: nutrition and lifestyle for a health pregnancy outcome. Journal of the American Dietetic Association, 108(3), 553-61.

Kamineni, R. (2005). The next stage of psychographic segmentation: usage of enneagram. Journal of American Academy of Business, 6(1), 315-20.

Kaplan, R. M., Bush, J. W., \& Berry, C C. (1976). Health status: types of validity and the index of well-being. Health Services Research, 11(4), 478-507.

Kassarjan, H.H. (1971). Personality and consumer behaviour: a review. Journal of Marketing Research, 8, 409-20.

Kaynak, E. \& Kara, A. (2001). An Examination of the relationship among consumer lifestyles, ethnocentrism, knowledge structures, attitudes and behavioural tendencies: A comparative study in two CIS states. International Journal of Advertising 20(4), 457-82.

Kegan, R. (1982). The Evolving Self. Cambridge, MA: Harvard University Press.

Kirsch, D. B., \& Avidan, A. Y. (2005). Pregnancy associated with daytime sleepiness and nighttime restlessness. Sleep Medicine, 6(5), 473-474.

Kolodinsky, J., \& Reynolds, T. (2009). Segmentation of overweight Americans and opportunities for social marketing. International Journal of Behavioural Nutrition and Physical Activity, 6(13), 6-13.

Kornelsen, J. (2002) Essences and imperatives: An investigation of technology in childbirth. Social Science and Medicine, 61(7), 1495-1504.

Kost, K., Landry, D. J., \& Darroch, J. E. (1998). Predicting Maternal Behaviors During Pregnancy: Does Intention Status Matter? Family Planning Perspectives, 30(2), 79-88.

Kotler, P., \& Levy, S.J. (1967). Broadening the concept of marketing. Journal of Marketing, 33(1), 10-15.

Kotler, P., \& Levy, S.J. (1969). A new form of marketing myopia: rejoinder to Professor Luck. Journal of Marketing, 33(3), 55-57. 
Kotler, P. (1979). Strategies for Introducing Marketing into Nonprofit Organizations. The Journal of Marketing, 43(1), 37-44.

Knudsen V.K, Orozova-Bekkevold I., Rasmussen L.B., Mikkelsen T.B., Michaelsen K.F., \& Olsen, S.F. (2004). Low compliance with recommendations on folic acid use in relation to pregnancy: is there a need for fortification? Public Health Nutrition 7(7), 843-50.

Kraft, F., \& Goodell, P. (1993). Identifying the health conscious consumer. Journal of Health Care Marketing, 13(3), 18-26.

Kralik, D., Visentin, K., van Loon, A. (2006). Transition: a literature review. Journal of Advanced Nursing, 55(3), 320-9.

Krisjanous, J., \& Love, M. (2002). First Steps toward Marketing to Maori. Paper presented at ANZMAC Conference, Melbourne.

Kruse, J., Zweig, S., \& LeFevre, M. (1988). Health locus of control and behaviours related to pregnancy. Family Medicine, 20(6), 422-5.

Kunz, L.H., \& King, J.C. (2007). Impact of maternal nutrition and metabolism on health of the offspring. Seminars in Fetal \& Neonatal Medicine, 12(1), 71-77.

Labs, S. M., \& Wurtele, S. K. (1986). Fetal health locus of control scale: Develpment and validation. Journal of Consulting and Clinical Psychology, 54(6), 814-819.

Laczinak, G \& Michie, D. (1979). The social disorder of the broadened concept of marketing, Journal of the Academy of Marketing Science, 7(3), 214-232.

Langley-Evans, S.C., \& Langley-Evans, A.J. (2002). Use of folic acid supplements in the first trimester of pregnancy. Journal of Social Health, 122(3), 181-6.

Lansing, J.B., \& Morgan, J.M. (1955). Consumer finances over the life cycle. In L. Clark (Ed.), Consumer Behaviour, 2, 36-50.

Larson, M.S. (1996). Sex roles and soap operas: What adolescents learn about single motherhood. Sex Roles, 35(1-2), 97. Retrieved September 26, 2010, from Research Library. (Document ID: 10290968).

Lastovicka J L. (1982). On the validation of lifestyle traits. Journal of Marketing Research, $19,126-38$. 
Lastovicka, J.L., Joachimsthaller, E. A., Bhalla, G., \& Scheurich, J. (1987). A lifestyle typology to model young male drinking and driving. Journal of Consumer Research, 14(2), 257-63.

Lawson, R., Rummel, A., Fiegler, B., \& Mueller-Heumann, G. (1990). A New Zealand lifestyle survey. New Zealand Journal of Business, 12, 22-35.

Lawson, R., \& Todd, S. (2002). Repackaging consumer lifestyles. Paper presented at the ANZMAC.

Lazer, W. (1963). Lifestyle concepts and marketing. In S. Greysser (Ed.), Toward Scientific Marketing (pp.243-52). Chicago: American Marketing Association.

Leap, N., \& Barclay, L. (2001). Midwifery education: Literature review and additional material. National Review of Nursing Education. United Kingdom: Commonwealth Department of Education and Training.

Lederman, R.P. (1996). Psychosocial adaptation in pregnancy: Assessment of seven dimensions of maternal development. New York, NY, US: Springer Publishing Co.

Leeuw, J. D. (2008). Handbook of Multilevel Analysis. New York: Springer.

Lemire, M., Paré, G., Sicotte, C., Harvey, C. (2008). Determinants of Internet use as a preferred source of information on personal health. International Journal of Medical Informatics, 77 (11), 723-34.

Levy, S.J., \& Kotler, P. (1969). Toward a broader concept of marketing's role in social order. Academy of Marketing Science, 7(3), 233-8.

Lewis, T. (2006). Seeking health information on the internet: lifestyle choice or bad attack of cyberchondria? Media, Culture \& Society, 28(4), 521-539.

Liamputtong, P., Halliday, J. L., Warren, R., Watson, L. F., \& Bell, R. J. (2003). Why do women decline prenatal screening and diagnosis? Australian women's perspective. Women \& Health, 37(2), 89-108.

Lifflander, A., Gaydos, L.M.D., \& Rowland Hogue, C.J. (2007). Circumstances of Pregnancy: Low Income Women in Georgia Describe the Difference between Planned and Unplanned Pregnancies. Maternal and Child Health Journal, 11(1), 81-9.

Lilley, R. (2007). What can social marketing offer public health? London: The Newbourne Group. 
Lindsay, L. (2004). The influence of the 'spaces of everyday life' on pregnancy health. The Canadian Geographer, 48(1), 35- 46.

Longhurst, R. (2005). (Ad)dressing Pregnant Bodies in New Zealand: Clothing, fashion, subjectivities and spatialities. Gender, Place \& Culture: A Journal of Feminist Geography, 12(4), 433-446.

Loudon, I. (2000). Tragedy of Childbed Fever. Oxford: Oxford University Press.

Lowenberg, J. S., \& Davis, F. (Writer) (1994). Beyond medicalisation-demedicalisation: the case of holistic health. Oregon: Blackwell Publishing Ltd.

Lu, M. 2007. Recommendations for preconception care. American Family Physician, 76(3), 397-401.

Lu, M. C., \& Halfon, N. (2003). Racial and ethnic disparities in birth outcomes: a lifecourse perspective. Maternal \& Child Health Journal, 7(1), 13-30.

Luck, D.J. (1969). Broadening the concept of marketing - too far? Journal of Marketing. 53-5.

Luna, I., Torres de Ardon, E., Mi Lim, Y., Cromwell, S. L., Phillips, L. R., \& Russell, C. K. (1996). The relevance of familism in cross-cultural studies of family caregiving. Western Journal of Nursing Research, 18(3), 267-283.

Lydon-Rochelle M.T., Krakowiak P., Hujoel P.P., \& Peters R.M. (2004). Dental care use and self-reported dental problems in relation to pregnancy. American Journal of Public Health, 94(5), 765-71.

McDermott L., Dobson A., \& Russell A. (2004). Changes in smoking behaviour among young women over life stage transitions. Australian New Zealand Journal of Public Health 28(4), 330-5.

McGlade, M.S., Saha, S., \& Dahlstrom, M.E. (2004). The Latina paradox: An opportunity for restructuring prenatal care delivery. American Journal of Public Health, 94(12), 2062-5.

McGrath, P. (2003). Benefits of participation in longitudinal qualitative research study. Monash Bioeth Rev, 22(1), 63-78.

McKenzie, P, J. (2001). Gaining access to everyday life information seeking, Library and Information Science Research, 23(4), 319-334.

McLeod, P.B., \& Ellis, J.R. (1983). Alternative Approaches to the Family Life Cycle in the Analysis of Housing Consumption. Journal of Marriage and Family, 45(3), 699-708. 
McQuillan, J., Greil, A.L., White, L., \& Casey, M.J. (2003). Frustrated fertility: infertility and psychological distress among women. Journal of Marriage and Family, 65(4), 1007-10.

Malhotra, N. K. (2007). Marketing Research : an applied orientation. Upper Saddle River, NJ: Pearson/Prentice Hall.

MacInnis, D. J., Moorman, C., \& Jaworski, B. (1991). Enhancing and measuring consumers' motivation, opportunity, and ability to process brand information from ads. Journal of Marketing, 55, 32-53. Maher, J. (2003). Rethinking women's birth experience: medical frameworks and personal narratives. Hecate, 29(2), 140-114.

Maibach E.W., Maxfield, A., Ladin, K. \& Slater, M. (1996). Translating health psychology into effective health action: The American Healthstyles Audience Segmentation Project. Journal of Health Psychology, 1, 261-278.

Maibach, E.W., Weber, D., Massett, H., Hancock, G.R., et al. (2006). Understanding consumer health information preferences development and validation of a brief screening instrument. Journal of Health Communication, 11(8), 717-36.

Malson, H., \& Swann, C. (2003). Re-producing 'woman's' body: Reflections on the (dis)place(ments) of 'reproduction' for (post)modern women. Journal of Gender Studies, 12(3), 191.

Mason, K. O., \& Lu, Y.-H. (1988). Attitudes toward Women's Familial Roles: Changes in the United States, 1977-1985. Gender and Society, 2(1), 39-57.

Mead, G.H. (1938). The Philosophy of the Act. Chicago: University of Chicago Press.

Mead, M. (1964). Continuities in Cultural Evolution. New Haven: Yale University Press.

Mercer, R.T. (2004). Becoming a mother versus maternal role attainment. Journal of Nursing Scholarship, 36(3), 226-32.

Miaoulis, G. (1989). Preadolescent pregnancy: A market segmentation perspective. Journal of Health Care Marketing, 9(2), 42.

Mikkelsen, T. B., Andersen, A.-M. N., \& Olsen, S. F. (2006). Pica in pregnancy in a privileged population: myth or reality. Acta Obstetricia et Gynecologica Scandinavica, 85(10), 1265-1266.

Miles, M.M., \& Huberman, A.M. (1994). An Expanded Sourcebook: Qualitative Data Analysis. London: Sage. 
Ministry of Health. (2006). Food and Nutrition Guidelines for Healthy Pregnant and Breastfeeding Women: A background Paper. Wellington: Ministry of Health.

Ministry of Health. (2004). Report on Maternity: Maternal and Newborn Information 2000-2001. Wellington: Ministry of Health.

Mitchell, V.M. (1994). How to identify psychographic segments. Marketing Intelligence and Planning, 12(7), 4-17.

Moran, C.F., Holt, V.L., \& Martin, D.P. (1997). What do women want to know after childbirth? Birth, 24(1), 27-34.

Morris, L.A., Swasy, J.L., \& Mazis, M.B. (1994). Accepted risk and alcohol use during pregnancy. Journal of Consumer Research, 21(1), 135-144.

Moschis, G.P. (1993). Gerontographics: a scientific approach to analyzing and targeting the mature market. The Journal of Consumer Marketing, 10(3), 43-53.

Moschis, G. P. (1996). Life stages of the mature market. American Demographics, 18(9), 44-49.

Mothersbaugh, D., Herrmann, R., Warland, R. (2003). Perceived time pressure and recommended dietary practices: the moderating effect of knowledge of nutrition. The Journal of Consumer Affairs, 27(1), 106-26.

Mowen, J.C., \& Carlson, B. (2003). Exploring the antecedents and the consumer behaviour consequences of the trait of superstition. Psychology and Marketing, 20(12), 1045-65.

Mueller-Heumann, G., \& Fiegler, B. (1979). A Lifestyle Segmentation Study of New Zealanders. Unpublished report by the University of Otago.

Müller, M. (1994). A questionnaire to measure mother-to-infant attachment. Journal of Nursing measurement, 2, 129-41.

Murphy, P.E., \& Staples, W.A. (1979). A modernized family life cycle. The Journal of Consumer Research, 6(1), 12-22.

Murphy Tighe, S. (2010). An exploration of the attitudes of attenders and non-attenders towards antenatal education. Midwifery, 26(3), 294-303.

Myers, J.E., Heazell, A.E.P., Jones, R.L., \& Baker, P.N. (2006). Obstetric management of teenage pregnancy. Fetala nd Maternal Medicine Review, 17(4), 317-25. 
Neal, C.M., Quester, P.G., \& Hawkins, D.I. (2002). Consumer Behaviour. Australia: McGraw-Hill.

Neill, J. (2002). Transcendence and transformation in the life patterns of women living with rheumatoid arthritis. Advanced Nursing and Science, 24(4), 27-47.

Netemeyer, R.G., Burton, S., \& Lichenstein, D.R. (1995). Trait aspects of vanity: Measurement and relevance to consumer behaviour. The Journal of Consumer Research, 21(4), 612-26.

Newman M.A. (1986). Health as Expanding Consciousness. St. Louis, MO: C.V. Mosby.

Newman, M.A. (1997). Evolution of the theory of health as expanding consciousness. Nursing Science Quarterly, 10(1), 22-5.

Nunnally, J. C., \& Bernstein, I. H. (1994). Psychometric theory (3rd ed.). New York: McGraw-Hill.

Oinas, F.J. (1993). Couvade in Estonia. The Slavic and East European Journal, 37(3), 339-45.

Osofsky, J.D. \& Osofsky, H.J. (1984). Psychological and developmental perspectives on expectant and new parenthood. Review of Child Development Research, 8, 37297.

Page L., \& McCandish, R. (2006). The New Midwifery: Science and Sensitivity in Practice. Philadelphia: Churchill Livingstone Elsevier.

Papps, E., \& Ramsden, I. (1996). Cultural Safety in Nursing: the New Zealand Experience. International Journal for Quality in Health Care, 8, 491-497.

Paquette, M., \& Devine, M. (2000). Dietary trajectories in the menopause transition among Quebec women. Journal of Nutrition Education, 32(6), 320-8.

Park, W.C., \& Mittal, B. (1985). A theory of involvement in consumer behaviour: problems and issues. In J.N Sheth (Ed.), Research in Consumer Behaviour, 1, 201-31.

Parkington, H.C., Coleman, H.A., Wintour, M.M., \& Tare, M. (2009). Pre-natal alcohol exposure: implications for cardiovascular function in the fetus and beyond. Clinical and Experimental Pharmacology and Physiology, 37(2), 91-98.

Pearlin, L.I., \& Schooler, C. (1978). The structure of coping. Journal of Health and Social Behaviour, 19(2), 2-21. 
Pearlin, L.I., Nguyen, K.B., Schieman, S., \& Milkie, M.A. (2007). The life-course origins of mastery among older people. Journal of Health and Social Behaviour, 48(2), 164-78.

Pender, N, J. (1987). Health promotion in nursing practice, $\left(2^{\text {nd }} E d\right)$. Norwalk: Appleton and Lang.

Pett, M., Lackey, N. \& Sullivan, J. (2003). Making Sense of Factor Analysis: The Use of Factor Analysis for Instrument Development in Health Care Research, USA: Sage Publications Inc.

Plummer, J. (1974). The concept and application of life style segmentation. Journal of Marketing, 38(9), 33-7.

Potomac. (1998). The women's audience is a myth effective segmentation requires more sophisticated approach. Healthcare PR and Marketing News, 7(19), 1.

Price, C.A. (2003). Professional women's retirement adjustment: the experience of reestablishing order. Journal of Aging Studies, 17(3), 341-55.

Priel, B., \& Besser, A. (2001). Bridging the gap between attachment and object relations theories: A study of the transition to motherhood. British Journal of Medical Psychology, 74, 85-100.

Prochaska, J.O., DiClemente, C.C., \& Norcross, J.C. (1992). In search of how people change: applications to addictive behaviours. The American Psychologist, 47(9), 1102-14.

Prochaska, J.O., \& DiClemente, C.C. (1993). Stages and processes of self-change of smoking: Toward an integrative model of change. Journal of Consulting and Clinical Psychology, 51, 390-5.

Pulley L., Klerman L.V., Tang H., \& Baker B.A. (2002). The extent of pregnancy mistiming and its association with maternal characteristics and behaviors and pregnancy outcomes. Perspectives on Sexual and Reproductive Health, 34(4), 206-11.

Quinla, J. D., \& Hill, D. A. (2003). Nausea and vomiting of pregnancy. American Family Physician, 68(1), 121.

Ragin, C. (2008). Redesigning social inquiry: Fuzzy sets and beyond. Chicago: University of California Press.

Revington, M. (2004). Secrets of the womb. New Zealand Listener, November, 20-26. 
Riley, L., \& Bowen, C. (2005). The sandwich generation: Challenges and coping strategies of multigenerational families. The Family Journal, 13(1), 52-8.

Risker, D.C. (1996). The health belief model and consumer information searches: toward an integrated model. Health Marketing Quarterly, 13(3), 13-26.

Rodgers, S., Chen, Q., Duffy, M \& Fleming, K, (2007). Media Usage as Health Segmentation Variables, Journal of Health Communication, 12(2), 105-119.

Rogers, R.W. (1975). A protection motivation theory of fear appeals and attitude change. Journal of Psychology, 91(1), 93-114.

Roodenburg, P. J., Wladimiroff, J. W., van Es, A., \& Prechtl, H. F. R. (1991). Classification and quantitative aspects of fetal movements during the second half of normal pregnancy. Early Human Development, 25(1), 19-35.

Root, R., \& Browner, C. (200). Practices of the pregnant self: compliance with and the resistance to prenatal norms. Culture, Medicine and Psychiatry, 25(2), 195-223.

Ross, D., Jones, J and Lynch, M. (2006). Toxoplasmosis, cytomegalovirus, listeriosis and preconception care. Maternal and Child Health Journal, 10(1), 189-193.

Rotter, J.B. (1954). Social Learning and Clinical Psychology. Englewood Cliffs, NJ: Prentice-Hall.

Rowntree, B.S. (1903). Poverty: A Study of Town Life. London: Macmillan.

Roy, C. (1997). Future of the Roy model: challenge to redefine adaptation. Nursing Science Quarterly, 10(1), 42-8.

Rubin, R. (1967). Attainment of maternal role. Part 1 processes. Nursing Research, 16, 237-45.

Rubin, R. (1975). Maternal tasks in pregnancy. Maternal-Child Nursing Journal, 4, 14353.

Rubin, R. (1984). Maternal identity and the Maternal Experience. New York: Springer.

Rudski, J. (2003). What does a "superstitious" person believe? Impressions of participants. The Journal of General Psychology, 130(4), 431-45. Rutherford, S Gallo-Cruz (2008). Selling the ideal birth: Rationalization and re-enchantment in the marketing of maternity care. Advances in Medical Sociology, 10, 75-98. 
Ryan, G.W., \& Bernard, R. (2000). Data management and analysis methods. In N.K Denzin \& Y.S Lincoln (Eds.), Handbook of Qualitative Research (2 ${ }^{\text {nd }}$ Ed.). London: Sage.

Saks, M. \& Allsop, J. (Eds.). (2007). Researching health: qualitative, quantitative and mixed methods. London: Sage.

Salmela-Aro, S., Nurmi, J., \& Halmesmaki, E. (2001). Psychosocial characteristics of women and their partners fearing vaginal birth. Journal of Obstetrics and Gynaecology, 108(5), 492-98.

Salmela-Aro, K. (2009). Personal goals and well-being during critical life transitions: The four C's--Channelling, choice, co-agency and compensation. Advances in Life Course Research, 14(1-2), 63-73.

Sagrestano, L. M., Feldman, P., Rini, C. K., Woo, G., \& Dunkel-Schetter, C. (1999). Ethnicity and Social Support During Pregnancy. American Journal of Community Psychology, 27(6), 869-898.

Sagrestano, L. M., Rodriguez, A. C., Carroll, D., Bieniarz, A., Greenberg, A., Castro, L., et al. (2002). A Comparison of Standardized Measures of Psychosocial Variables With Single-Item Screening Measures Used in an Urban Obstetric Clinic. Journal of Obstetric, Gynaecologic, \& Neonatal Nursing, 31(2), 147-155.

Sawyer, L.M. (1999). Engaged mothering: The transition to motherhood for a group of African American women. Journal of Transcultural Nursing, 10, 14-21. Selwyn, J. (2008) Fetal Development, in Focus on Early Childhood: Principles and Realities (eds M. Boushel, M. Fawcett and J. Selwyn), Blackwell Science Ltd, Oxford, UK. doi: $10.1002 / 9780470690321 . c h 2$

Scannapieco, F. A., Bush, R. B., \& Paju, S. (2003). Periodontal Disease as a Risk Factor for Adverse Pregnancy Outcomes. A Systematic Review. Annals of Periodontology, $8(1), 70-78$.

Schieman, S., Nguyen, K., \& Elliott, D. (2003). Religiosity, socioeconomic status, and the sense of mastery. Social Psychology Quarterly, 66(3), 202-220.

Schiffman, L., Bednall, D., O'Cass, A., Paladino, \& Kanuk, L. (2005). Consumer Behaviour ( $3^{\text {rd }}$ ed). NSW: Pearson.

Schmid, K.L., Rivers, S.E., Latimer, A.E., \& Salovey, P. (2008). Targeting or Tailoring?. Marketing Health Services, 32-37. 
Schnaninger, C.M., \& Danko, W.D. (1993). A conceptual and empirical comparison of alternative housing lifestyle cycle models. The Journal of Consumer Research, 19(4), 580-94.

Schnaninger, C \& Lee, D. H. (2002). A new full-nest classification approach. Psychology and Marketing, 19(1), 25-58.

Schneider, M.G., \& Forthofer, M.S. (2005). Associations of psychosocial factors with the stress of infertility treatment. Health and Social Work, 30(3), 183-191.

Shaikh, B. T., Haran, D., Hatcher, J., \& Azam, S. I. (2008). Studying health-seeking behaviours: Collecting reliable data, conducting comprehensive analysis. Journal of Biosocial Science, $40(1)$, 53-68.

Sharma, S., Durvasula, S., \& Dillon, W. R. (1989). Some results on the behavior of alternate covariance structure estimation procedures in the presence of nonnormal data. Journal of Marketing Research, 26(2), 214-221.

Shufeldt, L., Oates. B., \& Vaught, B. (1998). Is lifestyle an important factor in the purchase of OTC drugs by the elderly? Journal of Consumer Marketing, 15(2), $111-24$

Schumacher, K.L., \& Meleis, A.I. (1994). Transitions: A central concept in nursing. The Journal of Nursing Scholarship, 26(2), 119-27.

Selye, H. (1950). The Physiology and Pathology of Exposure to Stress. Oxford, England: Acta, Inc.

Sharma, S., Durvasula, S., \& Dillion, W.R. (1989). Some results on the behaviour of the alternate covariance structure estimation procedures in the presence of nonnormal data. Journal of Marketing Research, 26(2), 214-21.

Shaw, J. (2004). Labouring under Misapprehensions: The Paradoxical Role of Selfefficacy Beliefs in the Outcomes of a First Birth. PhD Thesis, Victoria University of Wellington.

Sideridis, G.D. (2007). The regulation of affect, anxiety, and stressful arousal from adopting mastery - avoidance goal orientations. Stress and Health, 24, 55-69.

Sigerist, H.E. (1941). Medicine and Human Welfare. New Haven: Yale University Press. 
Sin, L.Y.M., \& Yau, O.H.M. (2004). Female role orientation of Chinese women: Conceptualization and scale development. Psychology and Marketing, 21(12), 1033-1058.

Slater, M.D. (1996). Theory and method in health audience segmentation. Journal of Health Communication, 1, 267-83.

Slater, M.D., \& Flora, J.A. (1991). Health lifestyles: Audience segmentation analysis for public health interventions. Health Education and Behaviour, 18(2), 221-33.

Smith, W.(1956). Product differentiation and market segmentation as alternative market strategies. Journal of Marketing, 21, 3-8.

Smith, L. (1999). The WOMB (WOMen's views of Birth) antenatal satisfaction questionnaire: development, dimensions, internal reliability and validity. British Journal of General Practice, 49, 971-5.

Solomon, M., Bamossy, G., \& Askegaard, S. (2002). Lifestyle marketing. In Consumer Behaviour: a European Perspective ( $2^{\text {nd }}$ ed $)$. Essex: Prentice-Hall.

Sorce, P., Tyler, P.R, \& Loomis, L.M. (1989). Lifestyles of older Americans. The Journal of Consumer Marketing, 6(3), 53-63.

Speizer, I. S., Santelli, S. J., Afable-Munsuz, A., \& Kendall, C. (2004). Measuring factors underlying intendedness of women's first and later pregnancies. Perspectives on Sexual and Reproductive Health, 36(5), 198-205.

SPARC. (2003). Obstacles to Action. Retrieved from www.sparc.org.nz/researchpolicy/research-/obstacles-to-action.

Spielberger, C. D. (1983). Manual for the state-trait anxiety inventory. Palo Alto, CA: Consulting Psychologists Press.

Speizer, I. S., Santelli, S. J., Afable-Munsuz, A., \& Kendall, C. (2004). Measuring factors underlying intendedness of women's first and later pregnancies. Perspectives on Sexual and Reproductive Health, 36(5), 198-205.

Stark, M. A. (2001). Relationship of psychosocial tasks of pregnancy and attentional functioning in the third trimester. Research in Nursing \& Health, 24, 194-202.

Statistics New Zealand. Birth rates. Retrieved October, 2008, from http://search.stats.govt.nz/search?p=KK\&srid $=S 2 \% 2 d 4 \& l b c=$ statsnz\&ts =custom $\& p w=$ birth\&uid $=31890934 \&$ isort $=$ score $\& w=$ irth $\% 20$ rate $\& r k=1$ 
Steidel, A. G. L., \& Contreras, J. M. (2003). A New Familism Scale for Use with Latino Populations. Hispanic Journal of Behavioral Sciences, 25(3), 312-330.

Stein, Z., \& Susser, M. (2000). The risks of having children in later life. BMJ, 320(7251), 1681-1682.

Steptoe, A., \& Wardle, J. (2001). Locus of control and health behaviour revisited: A multivariate analysis of young adults from 18 countries. British Journal of Psychology, 92(4).659-672.

Stewart, D.E, \& Streiner, D. (1994). Alcohol drinking in pregnancy. General Hospital Psychiatry, 16(6), 406-12.

Stinson, K. M., Lasker, J. N., Lohmann, J., \& Toedter, L. J. (1992). Parents' Grief following Pregnancy Loss: A Comparison of Mothers and Fathers. Family Relations, $41(2), 218-223$.

Stojanovic, J. (2008). Midwifery in New Zealand 1904-1971, Advances in Contemporary Nursing, 30(2), 156-167.

Strang, V.R., \& Sullivan, P.L (1985). Body image attitudes during pregnancy and the postpartum period. Journal of Obstetric, Gynecologic and Neonatal Nursing, 14(4), 332-7.

Stremersch, S. (2008). Health and marketing: The emergence of a new field of research. International Journal of Research in Marketing, 25(4), 229-233.

Stutts, W. (2002). Physical activity determinants in adults: Perceived benefits, barriers, and self efficacy. American Association of Occupational Health Nurses Journal, Vol. 50(11), 499-507.

Sweeney, M.A., \& Gulino, C. (1987). The health belief model as an explanation for breast-feeding in a Hispanic population. Advances in Nursing Science, 9(4), 35-50.

Symons, D.D, \& Hausenblas, H.A. (2004). Women's exercise beliefs and behaviors during their pregnancy and postpartum. Journal of Midwifery and Women's Health, $49(2), 138-44$.

Szeverényi, P., Póka, R., Hetey, M., \& Török, Z. (1998). Contents of childbirth-related fear among couples wishing the partner's presence at delivery. Journal of Psychosomatic Obstetrics and Gynecology, 19(1), 38-43. 
Tam, J.L.M., \& Tai, S.H.C. (1998). Research note: the psychographic segmentation of the female market in greater China. International Marketing Review, 15(1), 61-73.

Tapscott, D. (1998). Growing up in the digital age: the rise of the net generation. Boston: McGraw Hill.

Tashakkori, A., \& Teddlie, C. (Eds.). (2003). Handbook of mixed methods in social and behavioral research. Thousand Oaks, CA: Sage.

Taylor, J. S. (2000). Of sonograms and baby prams: Prenatal diagnosis, pregnancy and consumption. Feminist Studies, 26(2), 391-418.

Te Puni Kokiri, Ministry of Maori Development. (2000). Progress towards closing the gaps between Maori and Non-Maori: A Report to the Ministry of Maori Affairs. Wellington, New Zealand: Te Puni Kokiri.

Tilikidou, I., \& Delistavrou, A. (2001). Utilisation of selected demographics and psychographics in understanding recycling behaviour. Greener Management International, 34, 75-93.

Todd, S., \& Lawson, R., \& Faris, F. (1998). A lifestyle analysis of New Zealand consumers. Asia Pacific Journal of Marketing and Logistics, 10(3), 30-47.

Todd, S., \& Lawson, R. (2001). Lifestyle segmentation and museum/gallery visiting behaviour. International Journal of Nonprofit \& Voluntary Sector Marketing, 6(3), 269-277.

Trigg, C., Peterson, K., \& Meekers, D. (1997). Psychographic predictors of risky sexual practice. South African AIDS News, 5(4), 13-5.

Tulman, L. \& Fawcett, J. (2003). Women's Health During and After Pregnancy: A Theory Based Study of Adaptation to Change. New York: Springer Publishing Co.

Tunc, T., Karadag, Y. S., Dogulu, F., \& Inan, L. E. (2007). Predisposing factors of restless legs syndrome in pregnancy. Movement Disorders, 22(5), 627-631.

Turner, R. \& Donald, L. (1999). The stress process and the social distribution of depression. Journal of Health and Social Behavior, 40, 374-404.

Veblen, T. (1979). The theory of the leisure class. Harmondsworth: Penguin. (Original work published 1899)

Villarreal, R., \& Peterson, R.(2009). The concept and marketing implications of Hispanicness. Journal of Marketing Theory and Practice. 17(4), 303-317. 
Vickers, M.H., Breier, B.H., Cutfield, W.S., Hofman, P.L., et al. (2000). Fetal origins of hyperphagia, obesity, and hypertension and postnatal amplification by hypercaloric nutrition. American Journal of Physiology, 42(1), 83.

Vickers, M.H., Breier, B.H., McCarthy, D., \& Gluckman, P.D. (2003). Sedentary behaviour during postnatal life is determined by prenatal environment and exacerbated by postnatal hypercaloric nutrition. American Journal of Physiology. Regulatory, Integrative and Comparative Physiology, 285, 271-3.

Villani, K., Wind, Y. (1975). On the usage of 'modified' personality trait measures in consumer research. Journal of Consumer Research, 2, 223-8.

Villarreal, R., Blozis, S.A., \& Wildaman, K.F. (2005). Factorial invariance of a PanHispanic familism scale. Hispanic Journal of Behavioural Sciences, 27(4), 409-25.

Vyncke, P. (2002). Lifestyle segmentation: from attitudes, interests, opinions, to values, aesthetic styles, life visions and media preferences. European Journal of Communication Copyright, 17(4), 445-63.

Wadsworth, M., Butterworth, S., Hardy, W, Kuh, D., Richards, M., Langenberg, C., Hilder W \& M. Connor. (2003). Making Health Communications Meaningful for Women: Factors That Influence Involvement, Social Science and Medicine, 57(11), 21932205.

Wagner, J., \& Hanna, S. (1983). The Effectiveness of Family Life Cycle Variables in Consumer Expenditure Research. The Journal of Consumer Research, 10(3), 281291.

Walker, L.O., Cooney, A.T., \& Riggs, M.W. (1999). Psychosocial and demographic factors related to health behaviors in the 1st trimester. Journal of Obstetric, Gynecologic and Neonatal Nursing, 28(6), 606-14.

Walker, S. N., Sechrist, K. R., \& Pender, N. J. (1987). The health promoting lifestyle profile: Development and psychometric characteristics. Nursing Research, 36(2), 76-81.

Wallace, D. A., Dodd, M. M., McNeil, D. A., Jocelyn Churchill, A., Oelke, N. D., Arnold, S. L., et al. (Writer) (2009). A Pregnancy Wellness Guide to Enhance Care Through Self-Assessment, Personal Reflection, and Self-Referral. Oregon: Blackwell Publishing Inc.

Wallston, K. A. (2005). The validity of the multidimensional health locus of control scales. Journal of Health Psychology, 10(4), 623-631. 
Wallston, K. A., Wallston, B. S., \& DeVellis, R. (1978). Development of the Multidimensional Health Locus of Control (MHLC) Scales. Health Education Monographs, 6, 161-170.

Walzer, S. (1995). Transition into motherhood: Pregnant daughters' responses to their mothers.

Ward, C., \& Masgoret, (2008). Attitudes toward Immigrants, Immigration, and Multiculturalism in New Zealand: A Social Psychological Analysis1. The International Migration Review, 42(1), 227-248.

Warren, S., \& Brewis, J. (2004). Matter over mind? Examining the experience of pregnancy. Sociology, 38(2), 219-236.

Weber, M. (1904). Die Protestantische Ethik und der 'Geist' des Kapitalismus. Archiv fur Sozialwissenschaft und SozialpoUtik, 20, 1-54.

Wedel, M., \& Kamakura, W. (2000). Market Segmentation: Conceptual and Methodological Foundations ( $2^{\text {nd }}$ Ed.). Dordrecht: Kluwer Academic Publishers.

Weinstein, N.D., Lyon, J.E., Sandman, P.M., \& Cuite, C.L. (1998). Experimental evidence for stages of health behavior adoption: The precaution adoption process model applied to home radon testing. Health Psychology, 17, 445-53.

Weinstein, N.D. \& Sandman, P.M. (1992). A model of the precaution adoption process: Evidence from home radon testing. Health Psychology, 11, 170-80.

Wells, W. D., \& Gubar, G. (1966). Life Cycle Concept in Marketing Research. Journal of Marketing Research, 3(4), 355-363.

Wells, W. (Ed.). (1974). Life Style and Psychographics. Chicago: American Marketing Association.

Wells, W. (1975). Psychographics: A Critical Review. Journal of Marketing Research, 12, 196-213.

Wheatley, S.L., Brugha, T.S., \& Shapiro, D.A. (2003). Exploring and enhancing engagement to the psychosocial intervention 'Preparing for Parenthood'. Archives of Women's Mental Health, 6, 275-85.

Whyte, C. J. E., Keller, S. N., Slater, M. D., Pbert, L., Chang, E., Druker, S., et al. (2008). Tailoring web-based interventions to young female smokers using audience segmentation. Journal of General Internal Medicine, 23, 227-228. 
Wilkes, R.E. (1995). Household Life-Cycle Stages, Transitions, and Product Expenditures. The Journal of Consumer Research, 22(1), 27-42.

Willcocks, S., \& Conway, T. (1998). Strategic marketing and clinical management in health care: a possible way forward. Journal of Management in Medicine, 12(2), 120-34.

Wilson, C.J., Deane, F.P., \& Ciarrochi, J. (2005). Can hopelessness and adolescents' beliefs and attitudes about seeking help account for help negation? Journal of Clinical Psychology, 61(12), 1525-39.

Wolburg, J.M, \& Pokrywczynski, J. (2001). A psychographic analysis of generation Y college students. Journal of Advertising Research, 41(5), 33-52.

Woodside, A.G., Nielson, R.L., Walters, F., \& Muller, G.D. (1998). Preference segmentation of health care services: The old fashioneds value conscious, affluents, and professional want-it-alls. Journal of Health Care Marketing, 8, 1424.

World Health Organisation. (1986). The Ottawa Charter for Health. Retrieved November, 2009, from https://www.who.int/healthpromotion/conferences/previous/ ottawa/en/.

World Health Organisation (1940). Definition of health. Retrieved March, 2008, from http://www.who.int/suggestions/faq/en/.

Wu, C.J., \& Chung, U.L. (2003). The decision-making experience of mothers selecting waterbirth. Journal of Nursing Research, 11(4), 261-7.

Xue, F., Holzman, C., Rahbar, M.H., Trosko, K., \& Fischer, L. (2007). Maternal fish consumption, mercury levels, and risk of preterm delivery. Environmental Health Perspective, 115(1), 42-7.

Yangihara, H., \& Yuan, K. (2005). Four improved statistics for contrasting means by correcting skewness and kurtosis. British Journal of Mathematical and Statistical Psychology, 58(2), 209-37. Yoo, G. (2006). Changing views on family diversity in urban Korea. Journal of Comparative Family Studies, 37(1), 59-74.

Zechmeister , I. (2001). Foetal images: the power of visual technology in antenatal care and the implications for women's reproductive freedom. Health care analysis, 9, 387-400. 